\title{
Flow Simulation of the Component Development Integration Facility Magnetohydrodynamic Power Train System
}

S.L, Chang, S.A. Lottes, J.X. Bouillard, and M. Petrick

Energy Systems Division

Argonne National Laboratory, 9700 South Cass Avenue, Argonne, Illinois 60439

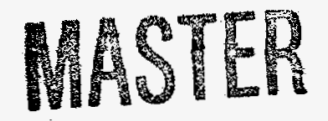

DISTRIBUTION OF THS DOCUEHENT IS UNLMRTE

November 1997

Work sponsored by United States Department of Energy, Assistant Secretary for Fossil Energy 


\section{DISCLAIMER}

This report was prepared as an account of work sponsored by an agency of the United States Government. Neither the United States Government nor any agency thereof, nor any of their employees, make any warranty, express or implied, or assumes any legal liability or responsibility for the accuracy, completeness, or usefulness of any information, apparatus, product, or process disclosed, or represents that its use would not infringe privately owned rights. Reference herein to any specific commercial product, process, or service by trade name, trademark, manufacturer, or otherwise does not necessarily constitute or imply its endorsement, recommendation, or favoring by the United States Government or any agency thereof. The views and opinions of authors expressed herein do not necessarily state or reflect those of the United States Government or any agency thereof. 


\section{DISCLAIMER}

Portions of this document may be illegible electronic image products. Images are produced from the best available original document. 


\title{
TABLE OF CONTENTS
}

\begin{abstract}
$\underline{\text { Page }}$
\end{abstract}
ACKNOWLEDGMENTS

viii

NOTATION

ix

ABSTRACT

1 INTRODUCTION

2 SEED UTILIZATION IN THE DESWIRL SECTION ....................................... 5

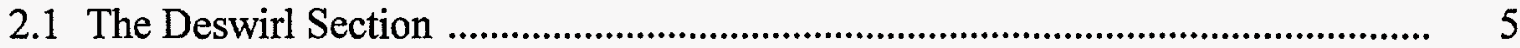

2.2 Multiphase 3-D Nonreacting Flow Computer Code ......................................... 5

2.2.1 Governing Equations ........................................................................... 6

2.2.2 Staggered Grid and Blocked Cells ........................................................ 8

2.2.3 Numerical Convergence and CPU Time ................................................ 9

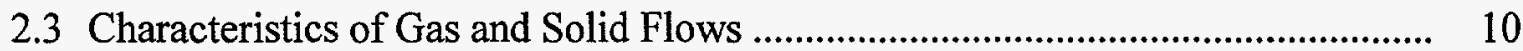

2.3.1 Gas Flow Characteristics ...................................................................... 11

2.3.2 Slag Flow Characteristics ................................................................. 13

2.3.3 Seed Flow Characteristics ............................................................... 16

2.4 Optimal Seed Utilization in the Deswirl Section ........................................ 19

3 ANALYSIS OF FLUID MIXING IN THE SECOND-STAGE COMBUSTOR ........ 21

3.1 The Second-Stage Combustor .................................................................. 21

3.2 Single-Phase 2-D Nonreacting Flow Computer Code ...................................... 22

3.3 Effects of Operating Conditions on Fluid Mixing ............................................. 27

3.3.1 Effects of Jet Angle ........................................................................... 29

3.3.2 Effects of Jet Momentum ............................................................... 34

3.3.3 Effects of Asymmetric Inlet Velocity Distribution ................................. 39

3.4 Comparison with Experiment ........................................................................ 41

4 REACTING FLOW IN THE SECOND-STAGE COMBUSTOR .......................... 43

4.1 Reacting Flow System ................................................................................. 43

4.2 Lumped Integral Combustion Submodel .................................................. 43

4.3 Effects of Operating Conditions on Combustion .............................................. 46

4.3.1 Effects of Jet Angle on Temperature Field ............................................. 47

4.3.2 Effects of Jet Angle on Combustion Efficiency ..................................... 54

4.4 Numerical Convergence and Stability ..................................................... 56 
5 IONIZATION OF SEED MATERIAL IN THE SECOND-STAGE COMBUSTOR.. 58

5.1 Multiphase Reacting Flow System ………..................................................... 58

5.2 Phenomenological Submodels for Multiphase Ionization Flows .......................... 59

5.2.1 Multiphase k- $\varepsilon$ Turbulent Submodel ......................................................... 59

5.2.2 Interfacial Drag and Heat Transfer Submodel ............................................ 59

5.2.3 Particle Evaporation Submodel .............................................................. 61

5.2.4 Gas Ionization Submodel ....................................................................... 61

5.3 Particle-Jet Interactions and Seed Vaporization ................................................... 67

5.3.1 Flow Patterns of Nonvaporizing Particles ................................................... 67

5.3.2 Flow Patterns of Vaporizing Particles ......................................................... 71

5.3.3 Effect of Particle Size on Seed Vapor Dispersion ....................................... 74

5.3.4 Effects of Seed Particle Inlet Location on Seed Vapor Dispersion .............. 77

5.4 Seed Ionization and Development of Electrical Conductivity Patterns

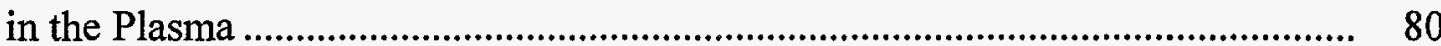

6 MULTIPHASE FLOW IN THE CONVERGING NOZZLE ....................................... 82

6.1 The Converging Nozzle ...................................................................................... 82

6.2 Flow Patterns in the Converging Nozzle ............................................................... 83

6.2.1 Effect of Nozzle Geometries on Flow Development .................................. 84

6.2.2 Residence Time and Heat Loss ............................................................ 88

6.3 Iron Oxide Injection in the Converging Nozzle .................................................... 90

$7 \quad$ ANALYSIS OF MHD CHANNEL OPERATION .................................................... 97

7.1 Three-Dimensional Code for MHD Generators and Diffusers .............................. 97

7.2 Channel Slag Leakage Model ............................................................................. 98

7.3 CDIF Channel Description .................................................................................. 101

7.4 CDIF Slagging Diagonal Channel Simulation ...................................................... 104

8 SIMULATION OF THE INTEGRATED MHD SYSTEM ....................................... 116

8.1 Computer Codes for the System Simulation …….................................................. 116

8.2 Flow Property Predictions of the Integrated MHD System .................................. 117

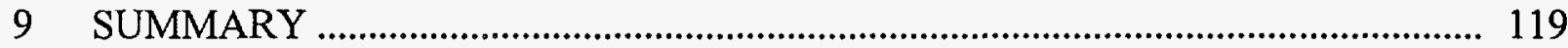

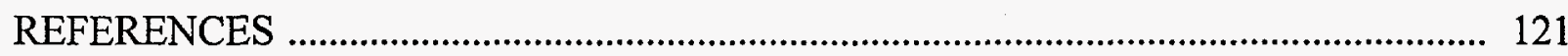




\section{LIST OF FIGURES}

No.

$\underline{\text { Page }}$

1 Sketch of the CDIF MHD Power Train System ……........................................................ 2

2 Various Views of the Deswirl Section ............................................................................... 6

3 Staggered Grid and Blocked Cells .......................................................................... 8

$4 \quad$ Blocked Grid System for the Deswirl Section ................................................................... 9

$5 \quad$ Gas Flow Patterns in Various $\mathrm{x}-\mathrm{z}$ Planes ................................................................... 11

6 Gas Flow Patterns in Various y-z Planes ................................................................. 13

$7 \quad$ Dispersion of Slag Particles in Various x-z Planes ..................................................... 14

8 Distribution of Slag Particle Number Densities, n, at the Exit ................................... 15

9 Dispersion of Seed Particles in Various x-z Planes ..................................................... 16

10 Distribution of Seed Particle Number Density, n, at the Exit ....................................... 17

11 Dispersion of Seed Particles by Intrusive Injection .................................................... 18

12 Percentages of Seed Particle Wall Deposition for Various Injection Locations .......... 20

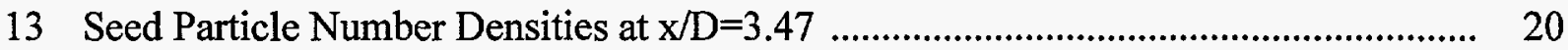

14 Schematic of the MHD Second-Stage Combustor .......................................................... 22

15 Computational Domain of 2-D Mixing Flow in the MHD

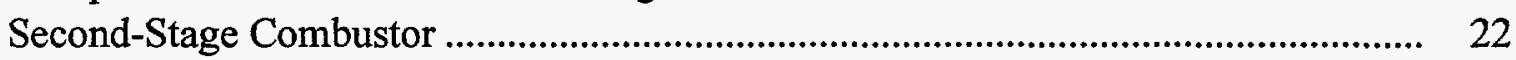

16 A 53 by 32 Grid System for the MHD Second-Stage Combustor .................................. 25

17 Jet Fluid Mixing Patterns and Turbulent Eddy Size - Baseline Case ........................... 28

18 Nonreacting Flow Patterns for Various Injection Angles ............................................ $\quad 30$

19 Jet Fluid Concentration of Nonreacting Flow for Various Injection Angles ................ 31

20 Effect of Injection Angle on Jet Fluid Mixedness in the Combustor ............................ 33

21 Effect of Injection Angle on Velocity and Concentration Profiles at the Combustor Exit ............................................................................................... 34

22 Effect of Injection Angle on Jet Fluid Mixedness at the Combustor Exit .................... 34

23 Effect of Injection Velocity on Velocity Field in the Combustor ................................. 36

24 Effect of Injection Velocity on Jet Penetration in the Combustor ................................. 37

25 Effect of Injection Velocity on Jet Fluid Mixedness in the Combustor ........................ 38

26 Effect of Injection Velocity on Jet Fluid Mixedness at the Combustor Exit ................ 39

27 Effect of Asymmetric Inlet Velocity on Nonreacting Flow Patterns

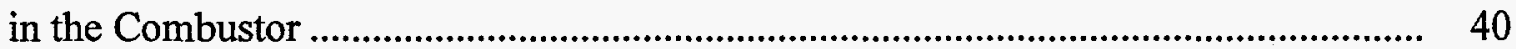

28 Effect of Injection Flow Rate on Jet Fluid Mixedness at the Combustor Exit ............. 42

29 Computational Domain of 2-D Combustion Flow in the MHD Second-Stage Combustor ..................................................................................... 43

30 Progress of Reaction Derived from Kinetics Calculation .............................................. 46

31 Combustion Flow Fields - 130-degree Injection Angle .............................................. 49

32 Combustion Flow Fields - 50-degree Injection Angle ................................................. 50

33 Effect of Injection Angle on Enthalpy and Fluid Mixedness in the Combustor .......... 51

34 Comparison of Combustion Flow Fields for 70- and 80-degree Injection Angles ....... 52

35 Effect of Injection Angle on Axial Enthalpy Mixedness in the Combustor .................. 53

36 Effect of Injection Angle on Combustion Efficiency in the Combustor ....................... 55 


\section{LIST OF FIGURES (cont.)}

No.

$\underline{\text { Page }}$

37 Effect of Injection Angle on Combustion Efficiency at the Combustor Exit ............... 55

38 Numerical Convergence of Integral and Differential Reaction-Rate Approaches ....... 57

39 Computational Domain for the Seed/Gas Flow in the Second-Stage Combustor ........ 58

40 Electron Concentration versus Temperature, Pressure, and Seed Mass Fraction ........ 63

41 Collision Frequency versus Temperature, Pressure, and Seed Mass Fraction ............. 66

42 Electric Conductivity versus Temperature, Pressure, and Seed Mass Fraction ........... 67

43 Particle Deposition Rate on Combustor Wall (No Evaporation) .................................. 68

44 Distribution of Nonvaporizing Particles in the Combustor .......................................... 69

45 Distribution of Nonvaporizing Particles in the Combustor for Each Size Group ........ 70

46 Gas and Particle Flow Patterns in the Combustor ........................................................ 71

47 Gas and Particle Temperature Distributions in the Combustor ...................................... 72

48 Distributions of Seed Particles and Vapor in the Combustor ......................................... 73

49 Particle Number Densities of Various Size Groups in the Combustor ........................... 74

50 Seed Vapor Concentration Profiles at the Combustor Exit for Various Mean Particle Sizes .................................................................................................. 75

51 Centerline Particle Number Densities in the Combustor for Each Size Group ........... 77

52 Seed Vapor Distribution in the Combustor for a Single-Cell Particle Injection ........... 78

53 Seed-Vapor Profiles in the Combustor for Various Single-Cell Injection Locations ... 79

54 Seed-Vapor Mixedness at the Combustor Exit for Various Single-Cell Injection Locations ................................................................................................. 80

55 Gas Ionization Patterns in the Combustor ................................................................ 81

56 Geometry of Nozzle \#1 and its Computational Domain .......................................... 83

57 Geometric Contours of Nozzles \#1, \#4, and \#5 .......................................................... 84

58 Development of Gas Pressure in Various Nozzles ..................................................... 86

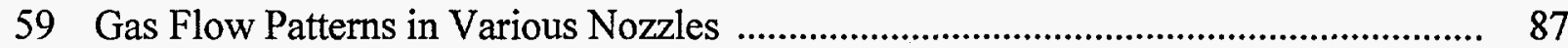

60 Particle Deposition in Various Nozzle Geometries ...................................................... 88

61 Gas and Particle Residence Times in the Nozzles ..................................................... 89

62 Gas and Particle Flow Patterns in Nozzle \#1 .............................................................. 92

63 Dispersion of Particles in Nozzle \#1 ............................................................................. 93

64 Distribution of Droplet Temperature in Nozzle \#1 ...................................................... 94

65 Iron-Oxide Particles Accumulation on Nozzle Side Walls ........................................... 95

66 Development of Particle Number Density in Nozzle \#1 ................................................ 95

67 Effects of Particle Size and Injection Velocity on the Uniformity of the Particle Dispersion............................................................................................. 96

68 Effects of Particle Size on the Particle Temperature at the Nozzle Throat ................... 96

69 Finite Segmenting Effects by Shorted Cathode Gaps ...................................................... 99

70 Typical CDIF 1 A 4 Nozzle and Channel Assembly ......................................................... 101

71 CDIF Axial Magnetic Field Distribution ............................................................... 104

72 Computed and Measured Axial Pressure Distribution (Unpowered Case) .................. 105

73 Calculated Axial Central Velocity, Temperature, and Mach Number ........................... 105 


\section{LIST OF FIGURES (cont.)}

No.

Page

74 Temperature Profile on a Cross Section $3.5 \mathrm{~m}$ from the Channel Inlet ....................... 106

75 Conductivity Profile on a Cross Section $3.5 \mathrm{~m}$ from the Channel Inlet ........................ 107

76 Computed Central Conductivity in the Axial Direction ............................................. 107

77 Slag Effect on the CDIF Generator Characteristics ........................................................ 109

78 Slag Effect on the Electrical Power Output of the CDIF Generator .............................. 110

79 Slag Effect on the Electrical Efficiency of the CDIF Generator .................................... 111

80 Computed Current Distribution of the CDIF Generator ................................................ 112

81 Velocity Profile on a Cross Section 2.2-m Downstream from the Nozzle Exit ........... 113

82 Computed Axial Distributions of Current Leakage, Hall Voltage, and Electric Field . 114

83 Computed and Measured Axial Distributions of Faraday Current Intensity ................ 114

84 Computed and Measured Axial Distributions of Faraday Potential .............................. 115

85 Computed and Measured Axial Distributions of Faraday Current Density ................. 115

86 Axial Development of Flow Properties in the CDIF MHD Power Train .................... 118

\section{LIST OF TABLES}

No.

Page

1 General Flow Conditions in the Deswirl Section ............................................................. 10

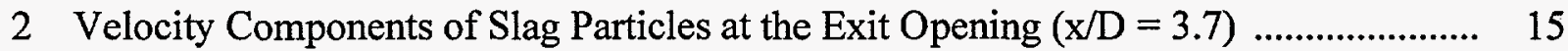

3 Exit Seed Particle Loading $(400 \mu \mathrm{m})$..................................................................... 19

4 Baseline Conditions of Nonreacting Flow in the MHD Second-Stage Combustor ....... 28

5 Relationship Between Jet Mass Flow Rate and Reynolds Number ................................. 42

6 Inlet Flow Properties of the Second-stage Combustor ................................................... 45

7 Molecular Weights and Specific Heats of the Lumped Species ...................................... 45

8 General Reacting Flow Conditions in the Second-Stage Combustor .............................. 47

9 Comparison of Predicted and Measured Potassium Gas Ionization ................................ 64

10 Electrons Contributed by Species in Flames ................................................................. 64

11 General Multiphase Flow Conditions in the Second-Stage Combustor .......................... 68

12 Nozzle Flow Conditions and Dimensions ................................................................ 84

13 Comparison of Flow Properties for Various Nozzle Geometries .................................... 90

14 Summary of Two-Phase Flow Properties in the \#1 Nozzle ............................................. 91

15 Typical $1 \mathrm{~A}_{4}$ Channel Parameters ........................................................................... 102

$161 \mathrm{~A}$ Generator Operating Conditions …….................................................................... 103

17 Typical Fuel/Reactant/Seed Compositions at CDIF ....................................................... 103

18 Computer Codes for the MHD System Simulation ....................................................... 117 


\section{ACKNOWLEDGMENTS}

This work was supported by the U.S. Department of Energy, Assistant Secretary for Fossil Energy, under Contract W-31-109-Eng-38. H. F. Chambers of Pittsburgh Energy Technology Center was the program manager. N. Swift was DOE's technical project officer. D. K. Schmalzer was Argonne's program manager. W. M. Swift was Argonne's project coordinator. The authors acknowledge G. F. Berry for his encouragement during the course of the study, as well as Q. Zhou for her assistance in preparing the figures. 


\section{NOTATION}

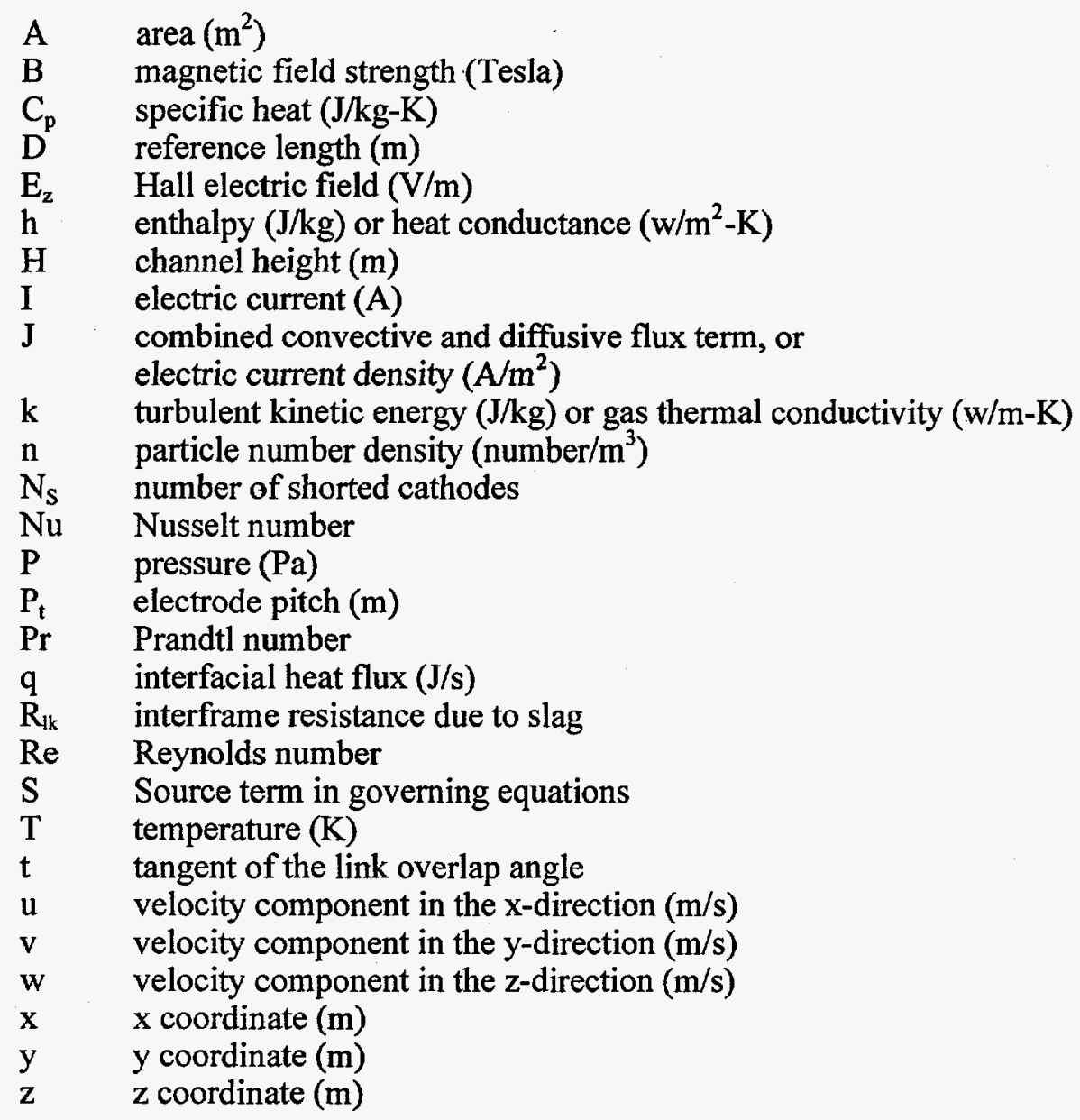

\section{Greek Symbols}

$\beta \quad$ Hall parameter

$\Gamma \quad$ property diffusivity (Pa-s)

$\varepsilon \quad$ turbulent dissipation rate $(\mathrm{J} / \mathrm{kg}-\mathrm{s})$

$\phi \quad$ reduced electric potential (V)

$\gamma \quad$ Diagonal angle (deg)

$\varphi \quad$ total electrical potential (V)

$\mu \quad$ viscosity ( $\mathrm{Pa}-\mathrm{s})$

$\theta \quad$ void fraction

$\rho \quad$ density $\left(\mathrm{kg} / \mathrm{m}^{3}\right)$

$\xi \quad$ general variable

$\begin{array}{ll}\text { Subscripts } \\ 1 & \text { load } \\ \text { s } & \text { solid phase } \\ \text { w } & \text { wall } \\ \text { x } & \text { x-direction } \\ \text { y } & \text { y-direction } \\ \text { z } & \text { z-direction } \\ \xi & \text { general variable }\end{array}$




\title{
Flow Simulation of the Component Development Integration Facility Magnetohydrodynamic Power Train System
}

\author{
by \\ S.L. Chang, S.A. Lottes, J.X. Bouillard, and M. Petrick
}

\begin{abstract}
This report covers application of Argonne National Laboratory's (ANL's) computer codes to simulation and analysis of components of the magnetohydrodynamic (MHD) power train system at the Component Development and Integration Facility (CDIF). Major components of the system include a 50-MWt coal-fired, two-stage combustor and an MHD channel. The combustor, designed and built by TRW, includes a deswirl section between the first and the second-stage combustor and a converging nozzle following the second-stage combustor, which connects to the MHD channel. ANL used computer codes to simulate and analyze flow characteristics in various components of the MHD system. The first-stage swirl combustor was deemed a mature technology and, therefore, was not included in the computer simulation. Several versions of the ICOMFLO computer code were used for the deswirl section and secondstage combustor. The MGMHD code, upgraded with a slag current leakage submodel, was used for the MHD channel.
\end{abstract}

Whenever possible data from the test facilities were used either to aid in calibrating parameters in the computer code, to validate the computer code, or to set base-case operating conditions for computations with the computer code. Extensive sensitivity and parametric studies were done on cold-flow mixing in the second-stage combustor, reacting flow in the second-stage combustor and converging nozzle, and particle-laden flow in the deswirl zone of the first-stage combustor, the second-stage combustor, and the converging nozzle. These simulations with subsequent analysis were able to show clearly in flow patterns and various computable measures of performance a number of sensitive and problematical areas in the design of the power train. The simulations of upstream components also provided inlet parameter profiles for simulation of the MHD power generating channel.

Among the more important results were the demonstration of the importance of oxidizer injection angle in the second-stage combustor, effects arising from the vaporization time of potassium seed particles, the inherent difficulties of controlling particle trajectories in a particleladen flow, and the consequent deleterious effects on performance and the shortened component operating time until repair or cleanout of the equipment is required. 


\section{INTRODUCTION}

A magnetohydrodynamic (MHD) power plant, which depends upon the interaction between magnetic fields and an electrically conducting fluid flow to generate electrical power, has attracted much interest in the utility industry because it can attain higher overall efficiency and produce fewer pollutants compared to a conventional coal-fired power plant [1-2]. Since the early 1980s, the U.S. Department of Energy has been sponsoring a national program for the development of a proof-of-concept MHD power plant in which industries, universities, and national laboratories were involved. Under this program, Argonne National Laboratory (ANL) provided technical support to the industries that were testing the MHD power train system at the Component Development and Integration Facility (CDIF).

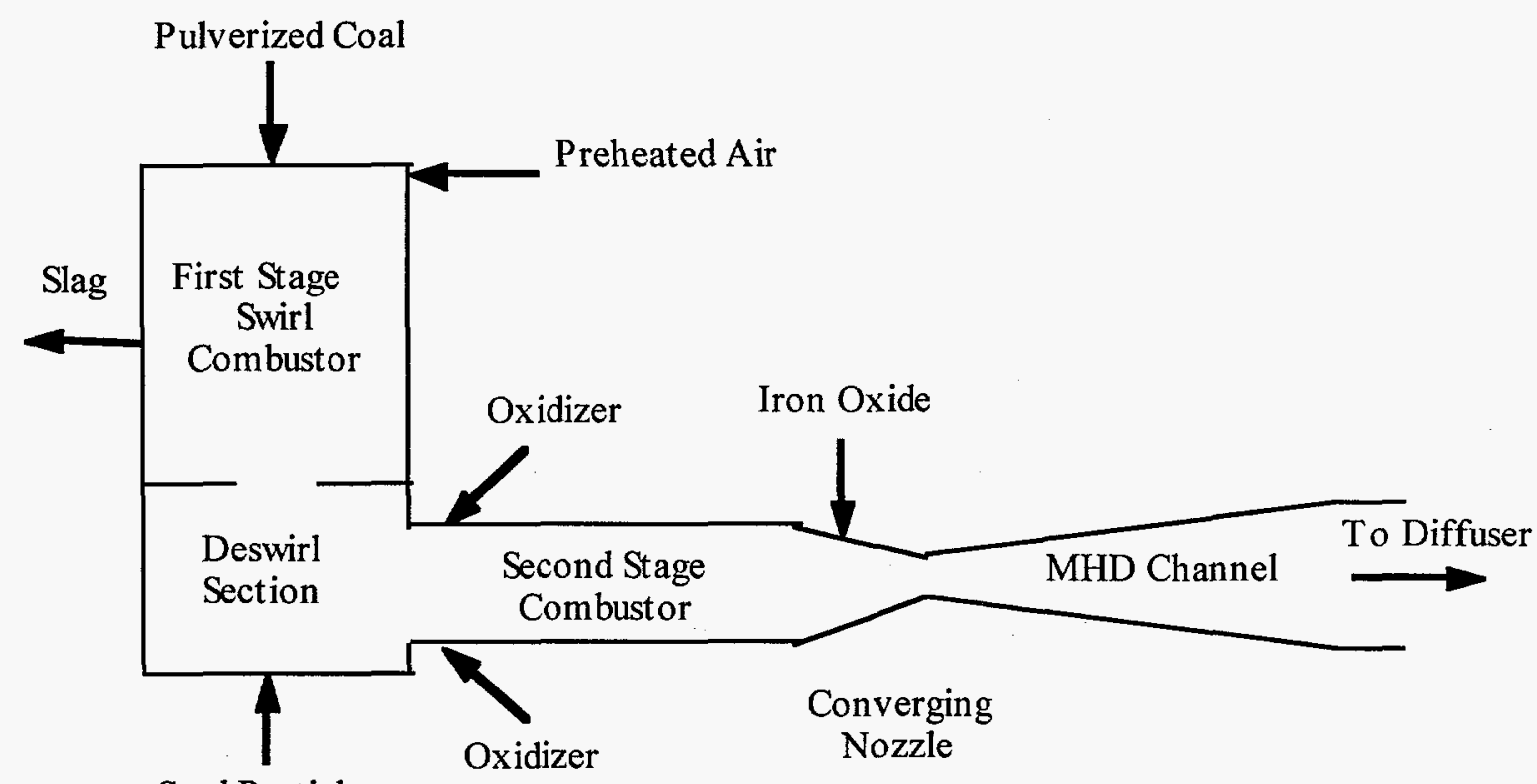

Seed Particles

Figure 1 Sketch of the CDIF MHD Power Train System (not to scale)

Figure 1 shows a sketch of the CDIF MHD power train system. Two major components of the MHD system are the two-stage combustor and the MHD channel. TRW designed and built the 50-MWt coal-fired combustor [3-4], consisting of a first-stage combustor, a deswirl section, a second-stage combustor, and a converging nozzle. The MHD channel was designed and built by Textron Defense Systems (formerly Avco Research Laboratory) [5]. The first-stage combustor is a swirl combustor in which pulverized coal is burned with preheated air at substoichiometric conditions. The swirl combustor produces coal gas while removing over $80 \%$ of slag by the swirling motion, lowering heat loss through the combustor walls, and minimizing $\mathrm{NO}_{\mathrm{x}}$ formation from the coal burning (fuel $\mathrm{NO}_{\mathrm{x}}$ ). Between the first- and second-stage combustors, there is a deswirl section in which the swirling coal-gas flow turns 90 degrees and enters the second-stage combustor with minimal velocity profile distortion. In the meantime, seed (potassium) particles are introduced into the coal-gas flow. A second-stage combustor, in which additional oxidizer 
(pure oxygen) is injected into the coal-gas flow, follows the deswirl section. The burning of the coal gas and oxidizer raises gas temperature to the level (about 3000K) where seed particles melt and vaporize and seed vapor ionizes. The combustion products leave the second-stage combustor as a plasma flow (electric conductivity higher than $8 \mathrm{mho} / \mathrm{m}$ ). In the following converging nozzle, the high-temperature plasma flow is accelerated to the sonic speed (about $1000 \mathrm{~m} / \mathrm{s}$ ) and a stream of iron oxide is injected along the bottom surface of the nozzle. The high-temperature, high-velocity plasma flows through the high magnetic field (about 6 tesla) of the channel. The electromagnetic interaction generates electric power. The iron oxide carried by the plasma flow should cover the cathode surfaces of the MHD channel and cut down the current leakage loss due to high voltage discharge. The MHD power train system was tested for an accumulated time of 525 operating hours at CDIF [6,7]. The system is capable of generating about $1 \mathrm{MWt}$ of electrical power.

The MHD power train system is complex, and development work on the system to enhance performance is difficult. Among important issues are penetration of oxidizer jets, mixing of coal gas and oxidizer, efficiency of second-stage combustion, utilization of seed material, uniformity of the plasma, iron oxide coverage, and erosion caused by iron oxide particles. Analysis using a computational fluid dynamics (CFD) computer code on modern computers is an efficient and cost-effective tool to help design better power train components, including combustors, nozzle, and MHD channel, because it can provide in-depth information about the combusting or conducting flow, and it provides the flexibility to experiment with a wide range of operating conditions at relatively low cost.

Computational results can provide output values of all flow properties at points on a grid mesh covering the entire flow field of a system component very easily and quickly, while detailed experimental measurements inside a coal-fired combustor, for example, may be difficult, if not impossible. At best, the measurements that can be made are very expensive because of equipment costs, operating costs, and limited equipment operating life in hostile environments. The highly sophisticated measurement equipment that may be required to collect even a relatively small amount of data may not be well differentiated, for example, with regard to velocity components of a particular size range of particles. As computing costs have dropped, however, and more sophisticated physical modeling has been developed for these complex flow systems, highly detailed results can be obtained relatively inexpensively that match experimental results fairly closely and provide data for constructing visualizations of complete flow field patterns, including velocity, temperature, pressure, species concentrations, and just about any other flow parameter of interest in the flow field.

Computed results can be readily used for such troubleshooting problems as particle deposition on the wall, or to search for optimum operating conditions, including maximizing efficiency or power density. Use of computer simulation can speed up the development cycle by reducing the number of prototypes that need to be built and tested. Computer simulation can be used to investigate all kinds of alternatives in combustor design, from different fuel/air mixtures to different combustor geometries, and to explore new design ideas quickly and economically. 
During the period when MHD power train modeling, simulation, and analysis was ongoing, a VAX computer was used at first for debugging and a CRAY XMP supercomputer was used for parametric studies at a cost of about $\$ 2000$ /month; however, during this period, the speed and capabilities of desktop computers developed to the point where complete computations could be run over a weekend at a cost of little more than the cost of electricity needed to power a couple of $100 \mathrm{Wt}$ light bulbs.

ANL has extensive experience in using CFD computer codes for the simulation and analysis of flow systems for many engineering applications. ANL's computer simulation expertise was used to study the CDIF MHD power train system. The computer simulation covers all components of the MHD system except the first-stage slagging combustor, which is considered by TRW to be mature technology. This report covers results obtained with both the two- and three-dimensional steady-state versions of the ICOMFLO computer code, and also a separate computer code MGMHD, are used to compute flow and electric power generation in the MHD channel. These codes were developed at ANL. The physics and structure of the ICOMFLO code are described in an accompanying report entitled, The MultiPhase Integral Reacting Flow Computer Code (ICOMFLO): User's Guide. The MGMHD code is documented in an earlier ANL report [8]. 


\section{SEED UTILIZATION IN THE DESWIRL SECTION}

A plasma flow with $1 \%$ (mass percentage) of potassium is needed to achieve design power output of the CDIF MHD power train. However, design verification tests at CDIF showed a much higher seed concentration $(1.7 \%)$ was required to achieve the design power output level [9]. The excessive seed injection has not only an economic impact but creates wall deposition and injector blockage problems. Reasons for the excessive seed injection include incomplete vaporization, nonuniform mixing, and wall deposition in the deswirl section, the second-stage combustor, and the nozzle. TRW suspected that the deswirl section where the seed is injected was of key importance in the excessive seed injection problem [10]. To help investigate the problem, ANL used a three-dimensional version of the ICOMFLO computer code to simulate and analyze gas and solid flows in the deswirl section [11].

\subsection{The Deswirl Section}

The deswirl section is located between the first and the second-stage combustors as shown in Fig. 1. The section is a short cylindrical tank with three openings: an inlet, a seed injection hole, and an exit. Figure 2 shows three views of the deswirl section: a rough three-dimensional view (Fig. 2a), a front view looking into the first-stage combustor (Fig. 2b), and a side view looking into the second-stage combustor (Fig. 2c). The deswirl section is roughly a cylinder of $0.762-\mathrm{m}$ diameter (the same as the first-stage combustor) and $0.457 \mathrm{~m}$ in length. The inlet is a circular area of $0.381-\mathrm{m}$ diameter (shaded area in Fig. $2 \mathrm{~b}$ ). The exit is a rectangular area of $0.284-\mathrm{m}$ height and $0.206-\mathrm{m}$ width (shaded area in Fig. 2c). Seed particles are injected pneumatically into the deswirl section from the front-end surface opposite the gas inlet surface, as shown in Fig. 2a.

The incoming flow is a swirling coal gas flow from the first-stage combustor. Seed particles are injected into the deswirl tank and mixed with the coal gas. To leave the deswirl section, the flow needs to turn a 90-degree angle and exit to the second-stage combustor. The turning of the flow should damp the swirl motion, and consequently, cut down the heat loss in the second-stage combustor.

\subsection{Multiphase 3-D Nonreacting Flow Computer Code}

A three-dimensional (3-D)multiphase hydrodynamic computer code was required to predict the gas/particle flow characteristics in the deswirl section because the multiphase flow in the deswirl section is a three-dimensional flow by nature. The computer code solving conservation equations of general flow properties for gaseous species and solid particles was expanded from a two-dimensional integral combustion code to a three-dimensional version of the same code. General conservation laws, expressed by elliptic-type partial differential equations, are used in conjunction with rate equations governing the mass, momentum, enthalpy, and species for a multiphase flow. Phenomenological submodels used to describe complex flow phenomena include two-parameter turbulence, interfacial drag and heat transfer, integral reaction, and 
particle/droplet evaporation submodels. In this investigation, only the turbulence and interfacial drag submodels were used. The turbulence submodel was modified to account for the effects of the particle phase. The interfacial submodel uses empirical correlations to calculate interfacial momentum and energy transfer.

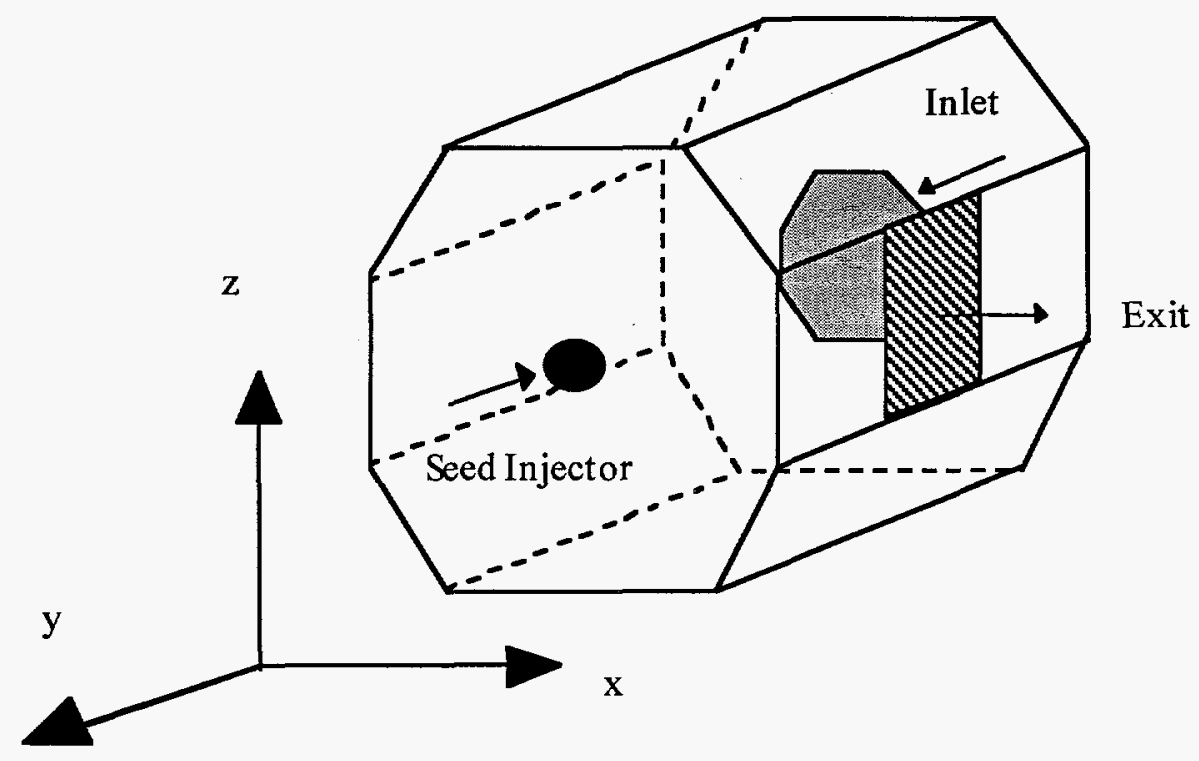

(a) Three-Dimensional View

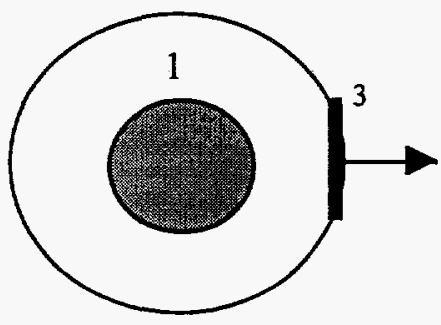

(b) Front View $(x-z)$

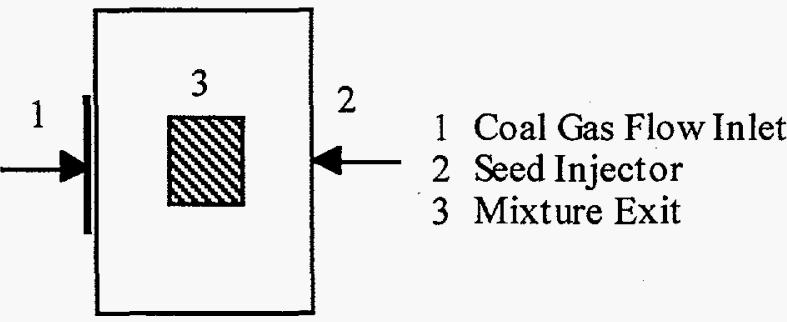

(c) Side View $(y-z)$

Figure 2 Various Views of the Deswirl Section

\subsubsection{Governing Equations}

The state of a multiphase flow is governed by the elliptic partial differential equations of fluid mechanics, including conservation of momentum, energy, and mass, with separate equations for chemical species conservation. These equations contain source and sink terms for interphase and intraphase property exchange rates. For convenience in numerical formulation, the governing transport equations for both gas and particle phases are put in a common form, 


$$
\frac{\partial J_{x}}{\partial x}+\frac{\partial J_{y}}{\partial y}+\frac{\partial J_{z}}{\partial z}=S_{\xi}
$$

where $J_{x}, J_{y}$, and $J_{z}$ are the three combined convective- and diffusive-flux terms in the $x, y$, and $z$ directions, and $S_{\xi}$ is the source term for a general flow property, $\xi$.

For the gas phase, the general flow variable is a scalar 1 in the continuity equation, a velocity component $\mathrm{u}, \mathrm{v}$, or $\mathrm{w}$ in a momentum equation, an enthalpy $\mathrm{h}$ in the energy conservation equation, a species concentration in a species conservation equation, or a turbulent kinetic energy $\mathrm{k}$, or a turbulent dissipation rate $\varepsilon$ in one of the turbulence transport equations. The three flux terms in Eq. (2.1) are expressed as

$$
\begin{aligned}
& \mathrm{J}_{\mathrm{x}}=\theta \rho \mathrm{u} \xi-\Gamma_{\xi} \frac{\partial \xi}{\partial \mathrm{x}} \\
& \mathrm{J}_{\mathrm{y}}=\theta \rho v \xi-\Gamma_{\xi} \frac{\partial \xi}{\partial \mathrm{y}} \\
& \mathrm{J}_{\mathrm{z}}=\theta \rho w \xi-\Gamma_{\xi} \frac{\partial \xi}{\partial \mathrm{z}}
\end{aligned}
$$

where $\theta$ is void fraction (fraction of gas volume), $\rho$ is density, and $\Gamma$ is effective diffusivity.

The source term in the gas phase continuity equation is the evaporation rate of particles. The source term in a momentum equation includes pressure gradient and momentum gain or loss through interfacial drag effects. The source term in the enthalpy equation includes the heat exchange between phases, heat of evaporation, and heat of combustion.

For the particle phase, the governing equations include equations of particle number density $(n)$, velocities $\left(u_{s}, v_{s}, w_{s}\right)$, and temperature $\left(T_{s}\right)$ for various particle sizes. The general flow property is a scalar 1 in the particle number density equation, a particle velocity component $u_{s}, v_{s}$, or $w_{s}$ in a particle momentum equation, and particle temperature $T_{s}$ in the particle energy equation. For each particle size group, the convective and diffusive flux terms are expressed as:

$$
\begin{aligned}
& \mathrm{J}_{\mathrm{x}}=\mathrm{nu}_{\mathrm{s}} \xi-\Gamma_{\xi} \frac{\partial(\mathrm{n} \xi)}{\partial \mathrm{x}} \\
& \mathrm{J}_{\mathrm{y}}=\mathrm{nv}_{\mathrm{s}} \xi-\Gamma_{\xi} \frac{\partial(\mathrm{n} \xi)}{\partial \mathrm{y}} \\
& \mathrm{J}_{\mathrm{z}}=\mathrm{nw}_{\mathrm{s}} \xi-\Gamma_{\xi} \frac{\partial(\mathrm{n} \xi)}{\partial \mathrm{z}}
\end{aligned}
$$

The diffusion term of the particle number density equation accounts for particle dispersion due to the interaction with the turbulence of the gas phase. Other interactions between phases are all included in the source terms. For example, a momentum sink in gas flow accounting for particle drag effects is also a momentum source for the particle flow. 


\subsubsection{Staggered Grid and Blocked Cells}

The governing non-linear partial differential equations, Eq. (2.1), are solved numerically. A staggered grid system was used for the numerical calculation. The grid system, as shown in Fig. 3, includes scalar, momentum, and blocked cells, with the gas velocity components stored on the scalar cell surfaces (or momentum cell points), all other physical quantities stored at the nodal points of each cell (or scalar cell), and the gas flow impermeable on the surface of a blocked cell. A momentum cell is extended by half a scalar cell when it is next to a blocked cell. The blocked cell technique enables the code to handle computational domains with complex geometries.

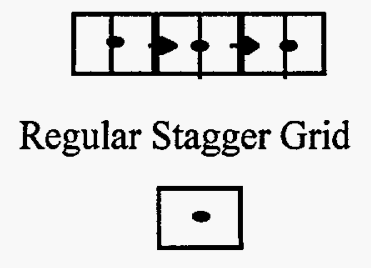

Scalar Cell

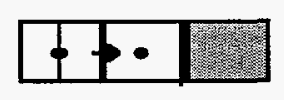

Right Blocked Cell

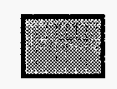

Blocked Cell

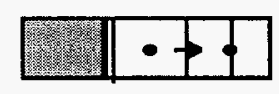

Left Blocked Cell

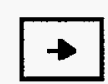

Momentum Cell

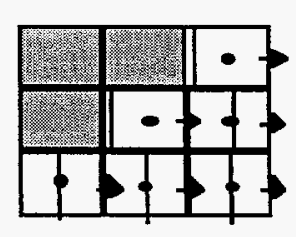

Corner Blocked Cells

\section{Figure 3 Staggered Grid and Blocked Cells}

The governing equations are transformed into algebraic equations by integrating over the computational cells, a procedure which achieves conservation of flow properties independent of grid size. Patankar's SIMPLER numerical algorithm [12] is modified to solve the discrete equations. These algebraic equations are solved using a line-by-line sweep combined with a tridiagonal matrix solver for computing a solution for each line. Particles are allowed to deposit on the walls by extrapolating the particle velocity to the walls. A momentum wall function is used to bridge the near wall boundary layer.

A $14 \times 13 \times 13$ grid system with blocked cells, as shown in Fig. 4, was selected for computing gas-particle flow characteristics in the deswirl section. The grid system consisted of 2366 scalar cells, 7098 momentum cells, and 9464 other grid points. Blocked cells were used to simulate the approximate contours of the deswirl boundaries, the inlet circle, the exit rectangle, and the injector hole. Figure $4 \mathrm{a}$ shows the inlet grid in an $\mathrm{x}-\mathrm{z}$ plane, in which the inlet circular area is represented by 45 open scalar cells. Figure $4 \mathrm{~b}$ shows an exit grid in another $\mathrm{x}-\mathrm{z}$ plane, in which the exit opening is represented by 25 scalar cells. Figure $4 \mathrm{c}$ shows the seed injection grid in a y-z plane, in which the intrusive injector is represented by blocked cell boundaries extended two scalar cells into the deswirl section. 

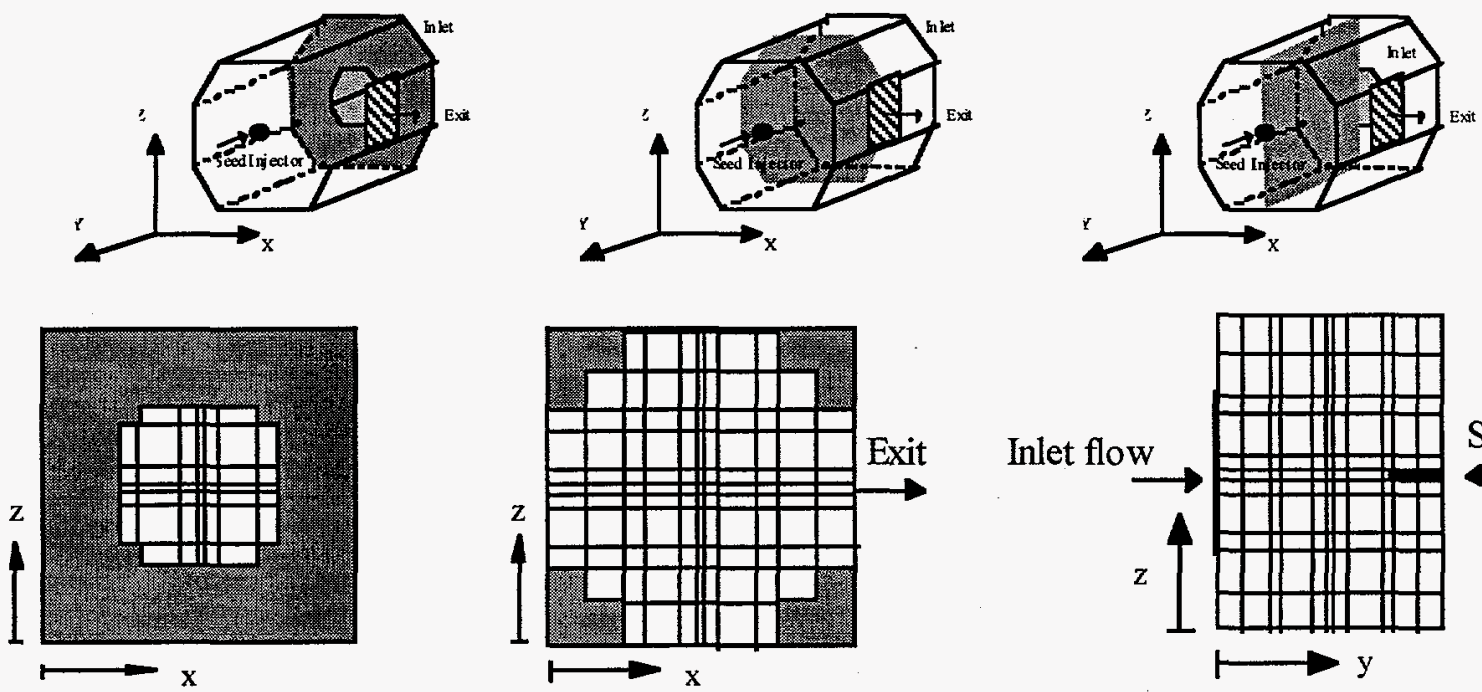

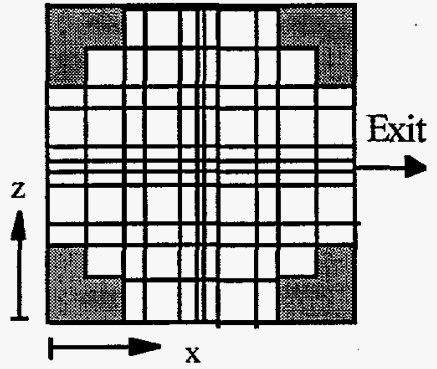

(b) Exit Plane

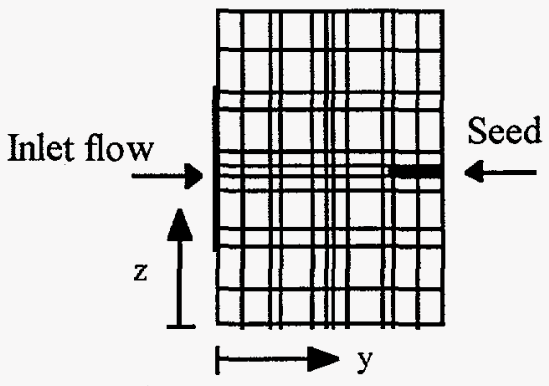

(c) Seed Injector Plane

(a) Inlet Plane

Figure 4 Blocked Grid System for the Deswirl Section

\subsubsection{Numerical Convergence and CPU Time}

Calculations of three-dimensional multiphase flow in the deswirl section using the selected grid system can be performed on an IBM-compatible personal computer. In performing preliminary calculations, numerical instabilities were encountered at the exit and at locations where particle-flow direction is reversed by the gas flow. At the exit, the usual free-boundary condition, i.e., zero flow property slope, is no longer valid because the flow is still developing. The problem was solved by specifying a small slope for flow properties and maintaining global mass balance. A new technique was developed to handle the instability problem caused by particle-flow reversal. At a particle-flow reversal location, calculation appeared divergent since particle loadings appeared to oscillate between a dense and a lean loading state, depending on the particle-flow direction. Computed particle-flow properties had only a single significant digit of accuracy, which was not acceptable because of high numerical uncertainty. Particle-flow properties on cell surfaces are commonly determined by interpolation of the computed values at neighboring scalar cells. At a location of particle-flow reversal, surface-flow velocity can be either positive or negative, which makes particles flow in or out of a cell and may cause oscillatory results. If a computational cell surface between two scalar points with opposing particle velocities is defined as impermeable, the oscillatory problem can be minimized. The new approach greatly improved numerical convergence in preliminary calculations. Computed particle properties had about three significant digits.

Determining the minimum amount of computational resources necessary to obtain good quality computational results with a relatively high confidence is a necessary step to control costs and allow a thorough parametric study to be done for a multiphase combustion flow simulation 
without exceeding the project budget. A convergence study was conducted for the MHD flow calculations to identify convergence levels for both gas and particle phases in which computed variables have converged to four or more decimal digits. Results of the sensitivity study indicate that if the average local mass residual is 9 orders of magnitude smaller than the inlet flow rate (or a convergence level of 9), the calculation has converged to a minimum of four decimal digits over nearly all of the grid points for all major gas flow variables. All calculations in the following discussion achieve the convergence level of 9 or above. A typical calculation took about 15 hours of CPU time on an IBM-compatible 386/25 personal computer running an OS/2 operating system. Calculation time can be reduced by using a restart technique which uses the solution of a previous calculation as an initial guess for the next calculation. By means of this technique, some calculations needed only about 8 hours of CPU time.

\subsection{Characteristics of Gas and Solid Flows}

An extensive parametric study was conducted to investigate gas-particle flow characteristics in the deswirl section, including flows of coal gas, slag particles, and seed particles. Coal gas is a mixture of the substoichiometric combustion products of coal and air in the first-stage combustion. Slag particles (or droplets) are the noncombustible mineral material in coal suspended in the coal gas. Coal-gas and slag particles enter the deswirl section from the inlet circular opening. Seed particles are injected in the deswirl section. The injector is located on a plane opposite to the inlet. Calculations were made for various injection locations and injector types, i.e., nonintrusive and intrusive.

\section{Table 1 General Flow Conditions in the Deswirl Section}

\begin{tabular}{|l|r|}
\hline Inlet Gas Pressure $(\mathrm{atm})$ & 5.77 \\
\hline Inlet Gas Temperature $(\mathrm{K})$ & 1974 \\
\hline Inlet Gas Flow Rate $(\mathrm{kg} / \mathrm{s})$ & 6.2 \\
\hline Inlet Gas Normal Velocity $(\mathrm{m} / \mathrm{s})$ & 40 \\
\hline Swirl Ratio & 1 \\
\hline Slag Particle Size $(\mu \mathrm{m})$ & 60 \\
\hline Slag Inlet Velocity $(\mathrm{m} / \mathrm{s})$ & 36 \\
\hline Slag Inlet Swirl Ratio & 1 \\
\hline Seed Particle Size $(\mu \mathrm{m})$ & $60-400$ \\
\hline Seed Injection Rate $(\mathrm{kg} / \mathrm{s})$ & 0.06 \\
\hline Seed Injection Velocity $(\mathrm{m} / \mathrm{s})$ & 100 \\
\hline
\end{tabular}

General flow properties of the deswirl section used in the parametric calculations are shown in Table 1. For the gas flow at the inlet, pressure is $5.77 \mathrm{~atm}$, temperature is $1974 \mathrm{~K}$, flow rate is $6.2 \mathrm{~kg} / \mathrm{s}$, normal velocity is $40 \mathrm{~m} / \mathrm{s}$, and swirl ratio is 1 . Note that the swirl ratio is the ratio of the maximum angular velocity to the normal velocity at the inlet. Slag- and seed-particle sizes range from 60 to $400 \mu \mathrm{m}$. Slag particles suspended in the gas flow have about $90 \%$ of the gas velocity at the inlet. Seed is injected from a front-end plane with a velocity of $100 \mathrm{~m} / \mathrm{s}$. Seed injection rate is $1 \%$ of the total gas mass-flow rate. 


\subsubsection{Gas Flow Characteristics}

Velocity vectors are plotted in Figs. 5a-d to show the gas flow patterns on four $\mathrm{x}-\mathrm{z}$ crosssectional planes at $y / D=0,0.46,1.11$, and 1.76 , respectively. Vector sense represents local flow direction and vector length represents flow speed. The inlet swirl flow has a maximum angular velocity of $40 \mathrm{~m} / \mathrm{s}$ at the edge of the inlet circular opening (Fig. 5a). The swirling flow appears to persist in the center portion but the strength of the swirling flow dissipates as it moves toward the front end plane $(y / D=2.22)$. The swirl ratio drops from 1 at inlet to about 0.5 at $y / D=0.46$ (Fig. 5b), to 0.1 at $y / D=1.11$ (Fig. 5c), and to almost zero at $y / D=1.76$ (Fig. $5 d$ ). When the gas reaches the end plane, it splits into two flows: one turns right to the exit direction and the other turns left. The left flow can only flow back to the inlet plane because there is no opening in the left side of the deswirl section, which creates a recirculating flow in this region.
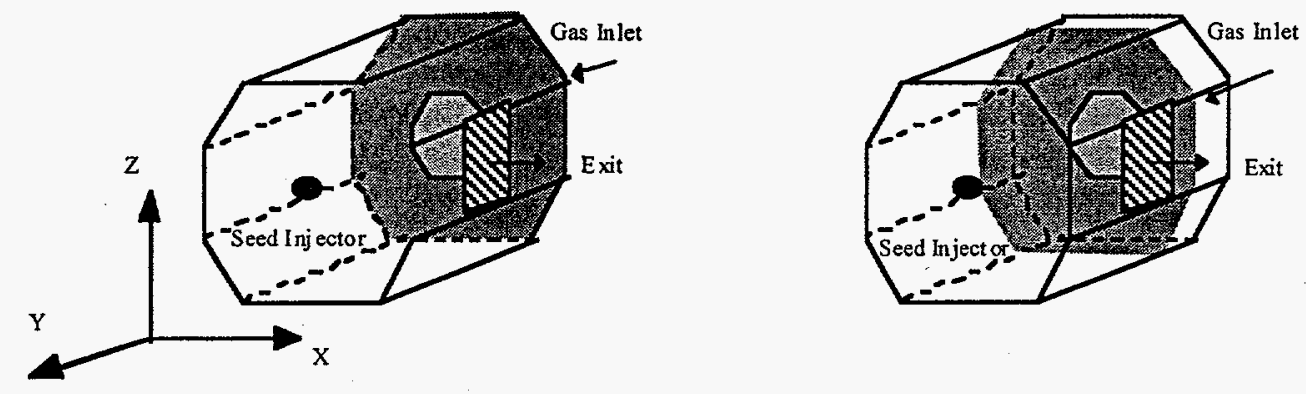

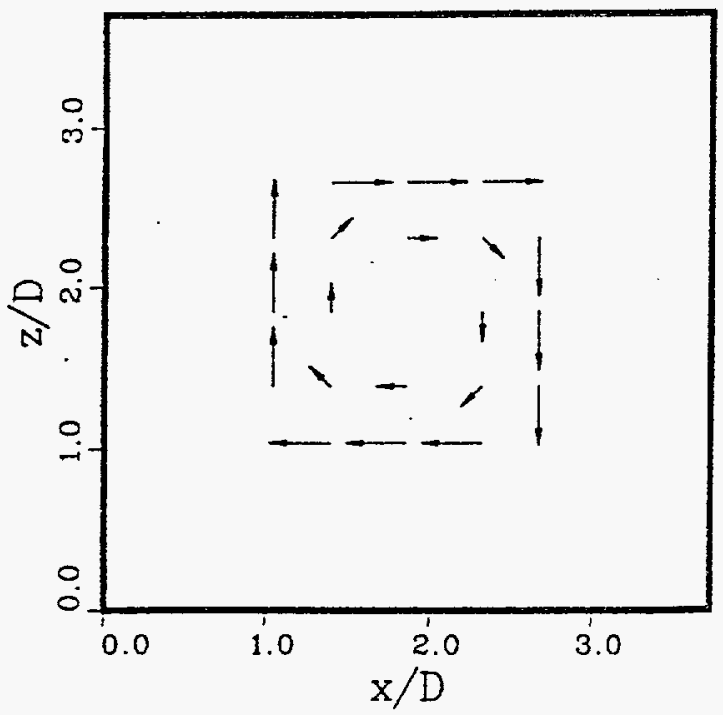

(a) $y / D=0.0$

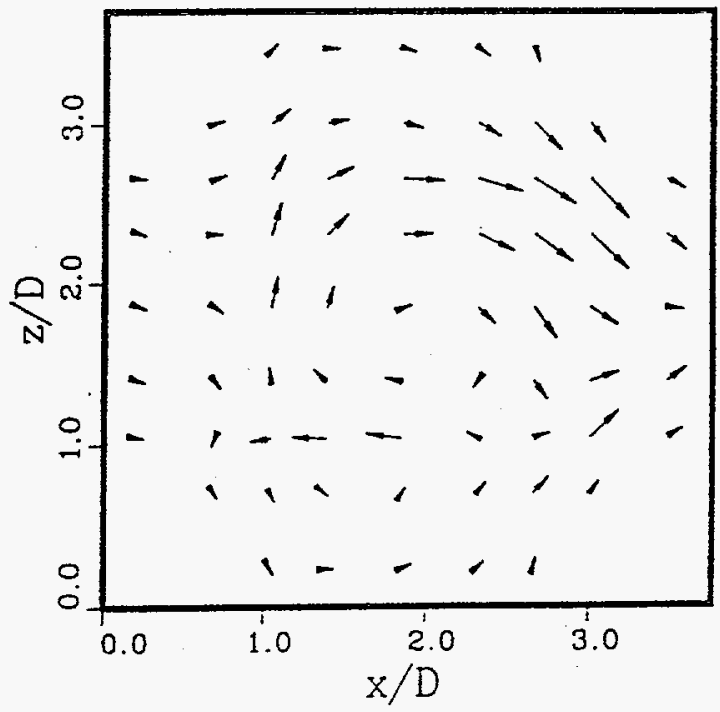

(b) $y / D=0.46$

Figure 5 Gas Flow Patterns in Various x-z Planes 

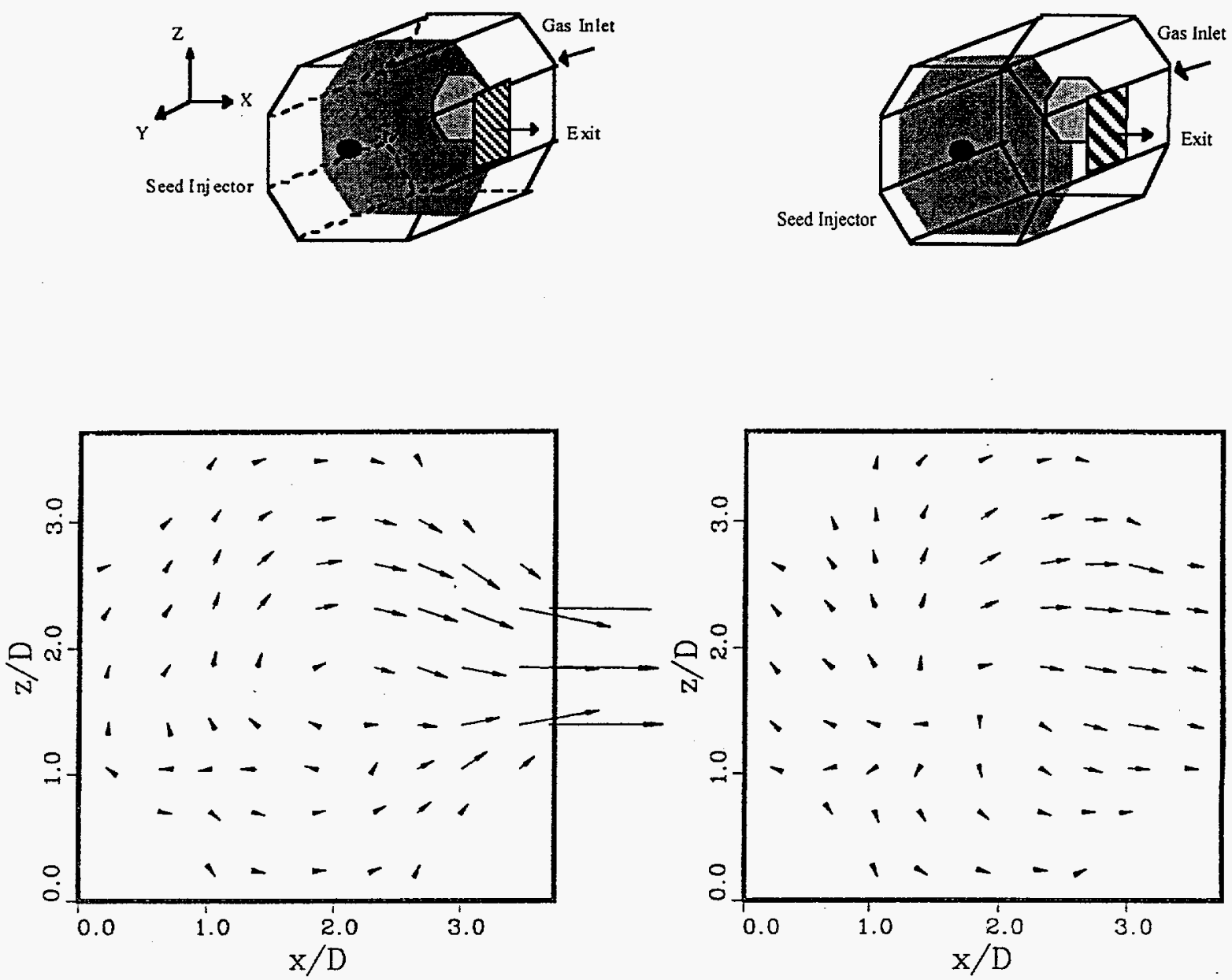

(c) $\mathrm{y} / \mathrm{D}=1.11$

(d) $y / D=1.76$

Figure 5 Gas Flow Patterns in Various x-z Planes (cont.)

The gas flow patterns in two $y-z$ cross-sectional planes at $x / D=1.85$ and 3.47 are shown in Figs. $6 \mathrm{a}$ and $6 \mathrm{~b}$, respectively. In these figures, two vortices are found; one is in the top portion and the other in the bottom portion. Due to the effect of the inlet swirl motion, the two vortices are uneven and asymmetric in the z-direction. At the exit, the upper vortex is larger than the lower vortex. The u-velocity distribution is asymmetric in both the $y$ - and z-directions. Exit $\mathrm{u}$-velocities at the far end $(\mathrm{y} / \mathrm{D}=1.44)$ are higher than those at the near end $(\mathrm{y} / \mathrm{D}=0.78)$. At $\mathrm{z} / \mathrm{D}=1.86$, the degree of asymmetry in the $y$-direction is about $10 \%$. Due to the inlet swirl motion, $\mathrm{u}$-velocities in the upper portion $(\mathrm{z} / \mathrm{D}=2.31)$ are higher than those in the lower portion $(z \mathrm{D}=1.39)$. The degree of asymmetry in the z-direction is also about $10 \%$. 

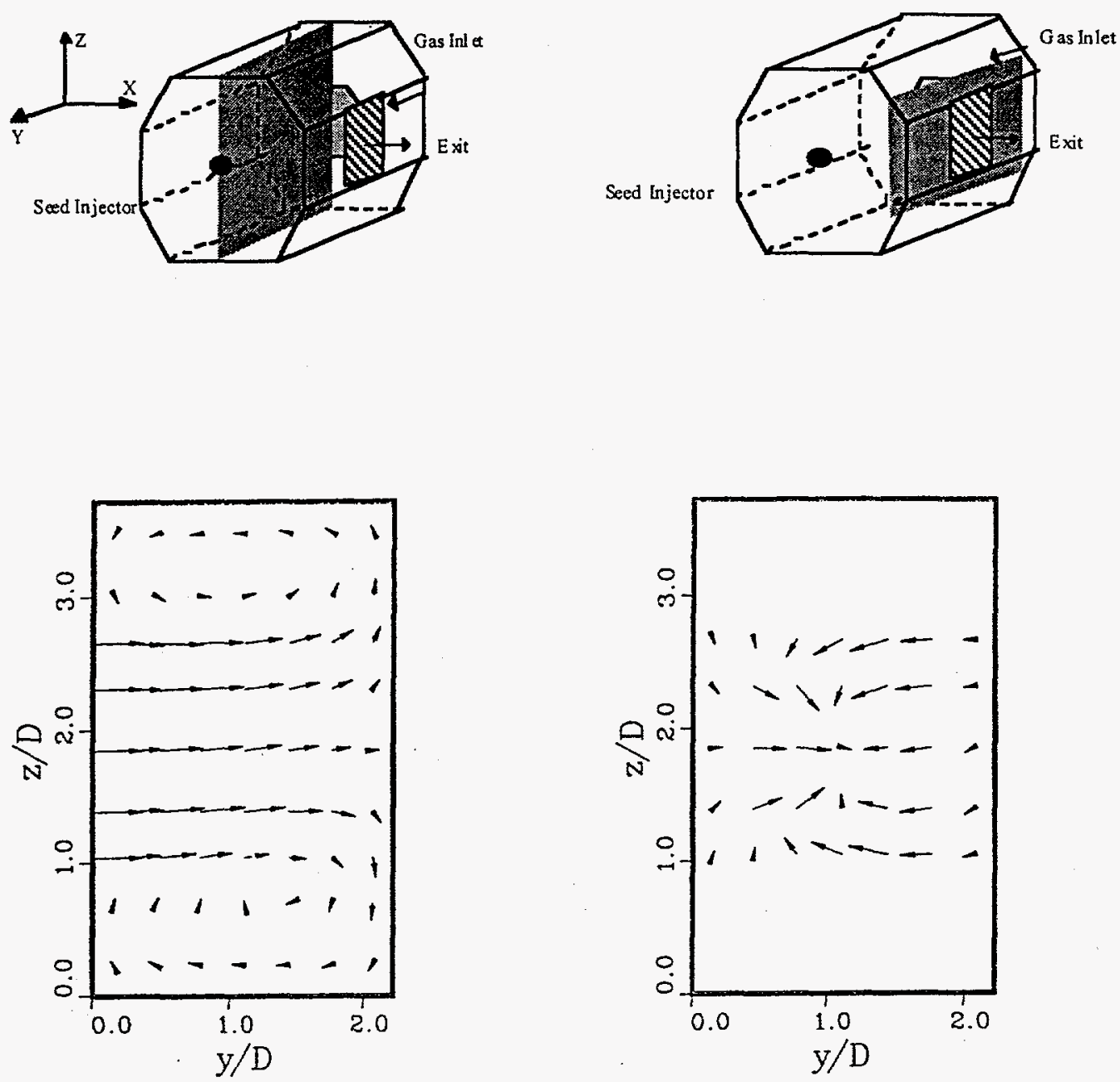

(a) $x / D=1.85$

(b) $x / D=3.47$

Figure 6 Gas Flow Patterns in Various y-z Planes

\subsubsection{Slag Flow Characteristics}

Slag particles (or droplets) which are suspended in the coal-gas flow enter the deswirl section from the inlet circular area. Calculations were made to study flow characteristics of slag particles in the deswirl section. In these calculations, the following assumptions were made: slag particle size is $60 \mu \mathrm{m}$, slag inlet normal velocity is $36 \mathrm{~m} / \mathrm{s}$, particle swirl ratio is 1 , and inlet normalized particle-number density is 0.18 . Note that a reference particle-number density is arbitrarily chosen as $10^{8} \mathrm{\#} / \mathrm{m}^{3}$. Contours of computed slag particle-number density on two $\mathrm{x}-\mathrm{z}$ cross sections at $y / D=1.11$ and 1.76 were plotted in Figs. $7 \mathrm{a}$ and $7 \mathrm{~b}$, respectively. The particlenumber density contour numbers in the figure were normalized by the reference value. 

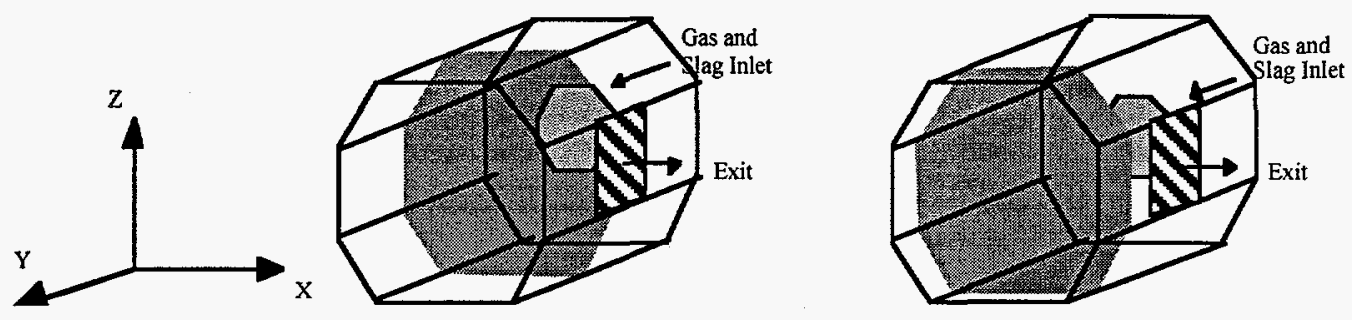

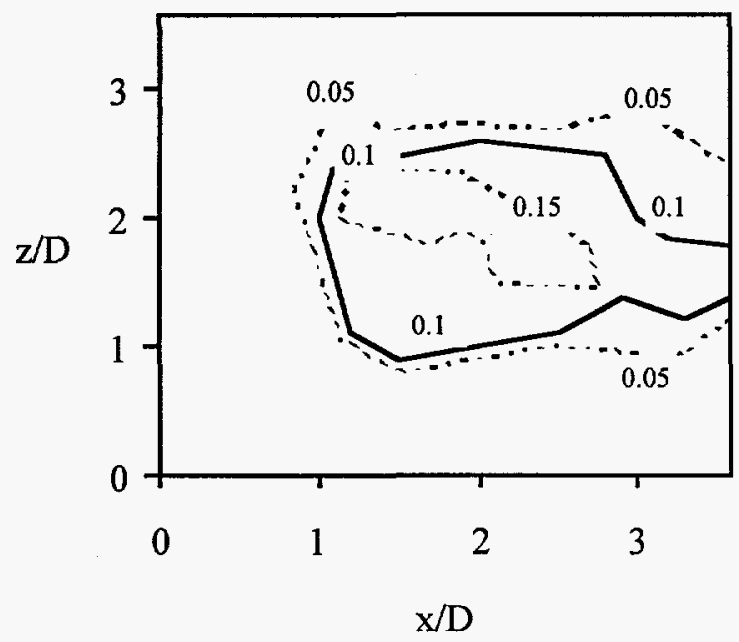

(a) $\mathrm{y} / \mathrm{D}=1.11$

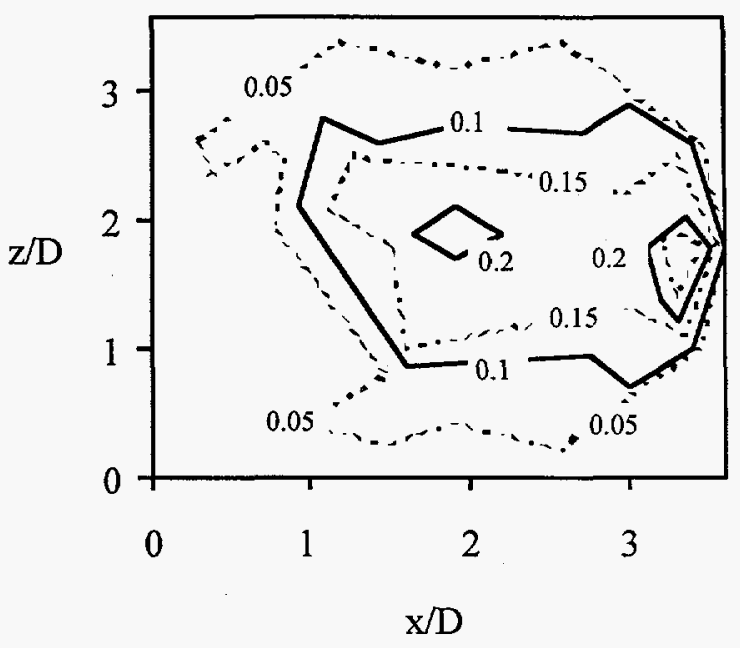

(b) $\mathrm{y} / \mathrm{D}=1.76$

Figure 7 Dispersion of Slag Particles in Various x-z Planes

Flow characteristics of slag particles are generally similar to gas flow characteristics because the particles are carried into the deswirl section by the gas flow. However, particles are slow to react to gas flow changes caused by boundaries due to their inertia, and some collide and deposit on a wall surface. After entering the deswirl section, slag particles are driven by three major forces: the inertial force to move in a general direction toward the seed injector plane (or end plane), a centrifugal force by the $y$-swirl motion (the vortex vector pointing in the $y$ direction), and a drag force generated by velocity differences between particle and gas flow. Due to the centrifugal force from the y-swirl motion, slag particles move from the center region toward the walls. Near the exit, some slag particles are carried by the accelerating gas flow through the exit and some overshoot the exit and continue to move toward the end plane. At $\mathrm{y} / \mathrm{DE}=1.11$, Fig. 7 a shows that some slag particles flow through the exit. At $\mathrm{y} / \mathrm{D}=1.76$, Fig. $7 \mathrm{~b}$ shows that some slag particles concentrate in the lower right side of the deswirl section due to a combined effect of exit flow and inlet swirl and that many deposit on the wall. When slag particles move near the end plane, some particles following the gas flow are split into two streams, and some are driven by the inertia force and deposit on the end wall. The split streams form secondary vortices in the $\mathrm{x}$ - and $\mathrm{z}$-directions. Since gas motion in the deswirl section changes direction and particles need time to adjust to the direction change of the gas flow through drag force, many slag particles collide with the side walls. By integrating over side wall 
surface in the deswirl section, the percentage of incoming slag particles which deposit on the walls is computed as $63 \%$. In other words, $37 \%$ of slag particles from the first-stage deswirl section escape to the second-stage combustor and downstream MHD components.

Slag flow at the exit is neither uniform nor parallel. Table 2 summarizes normalized velocity components of slag particles at the exit. The reference velocity is the average gas velocity at the exit, $92 \mathrm{~m} / \mathrm{s}$. Average u-velocity is about $50 \mathrm{~m} / \mathrm{s}$. Due to the inertia of the inlet momentum, many v-velocities are positive, with a maximum value of $27 \mathrm{~m} / \mathrm{s}$ at $\mathrm{y} / \mathrm{D}=0.78$ and $z / D=1.86$. The $w$-velocities are smaller than the v-velocities and have positive values in a lower portion, $z / D=1.39$, and negative values in the upper portion, $z / D=1.86$ or 2.31 . Because of large cross-stream velocity components in the $y$ - and z-directions, exit slag concentration is highly non-uniform, as shown in Fig. 8. Average exit normalized slag particle number density, $\mathrm{n}$, is about 0.1 , and the spatial deviation in the exit opening is $40 \%$.

Table 2 Velocity Components of Slag Particles at the Exit Opening $(x / D=3.7)$

\begin{tabular}{|c|c|c|c|c|}
\hline Velocity & & \multicolumn{3}{|c|}{$\mathrm{z} / \mathrm{D}$} \\
\cline { 3 - 5 } Direction & $\mathrm{y} / \mathrm{D}$ & 1.39 & 1.86 & 2.31 \\
\hline $\mathrm{u}$ & 0.78 & 0.565 & 0.541 & 0.560 \\
\hline & 1.11 & 0.538 & 0.508 & 0.526 \\
\hline & 1.44 & 0.573 & 0.561 & 0.572 \\
\hline $\mathrm{V}$ & 0.78 & 0.192 & 0.294 & 0.165 \\
\hline & 1.11 & 0.090 & 0.258 & 0.074 \\
\hline & 1.44 & -.086 & 0.132 & -.076 \\
\hline $\mathrm{w}$ & 0.78 & 0.107 & -.059 & -0.144 \\
\hline & 1.11 & 0.086 & -.044 & -0.107 \\
\hline & 1.44 & 0.009 & -.025 & -0.022 \\
\hline
\end{tabular}

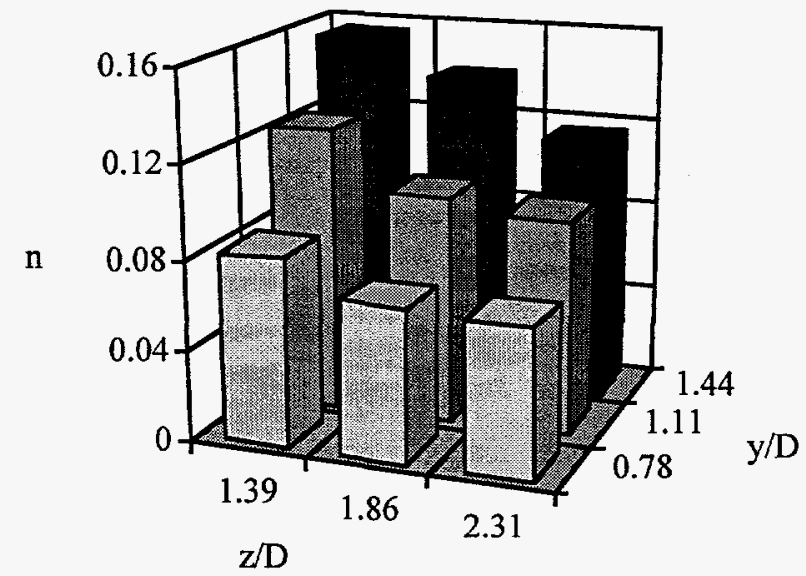

Figure 8 Distribution of Slag Particle Number Densities, n, at the Exit 


\subsubsection{Seed Flow Characteristics}

Calculations of seed particle flow characteristics in the deswirl section were made for various seed injection methods and locations on the end wall. The types of seed injection include nonintrusive and intrusive. These calculations neglect the effects of slag particles and seed-particle evaporation.

Contours of normalized seed particle-number density, $\mathrm{n}$, in two $\mathrm{x}-\mathrm{z}$ cross-sections are plotted in Fig. 9, assuming the seed is injected from a flush-mounted injector in the center of the end plane $(x / D=1.85, y / D=2.21$, and $z / D=1.85)$. Figure 9 a shows the contours in the $x-z$ cross-section at $y / D=1.76$ near the injection plane, and Fig. $9 \mathrm{~b}$ shows those in an exit plane at $y / D=1.11$. Since seed particles are injected against the incoming gas flow, a majority of the particles are pushed to the right and in particular the lower right side of the deswirl section by the right splitting gas stream. Many deposit on the walls (Fig. 9a) and some flow through the exit (Fig. 9b). Particles that miss the exit plane are mainly in the lower right quadrant of the deswirl section, and many deposit on the inlet wall at $y / D=0$. About three-quarters of the seed particles deposit on the walls of the deswirl section; the rest enter the second-stage combustor in a highly non-uniform manner as shown in Fig. 10. Seed particles leaving the deswirl section concentrate in the central right portion of the exit (looking into the second-stage combustor).
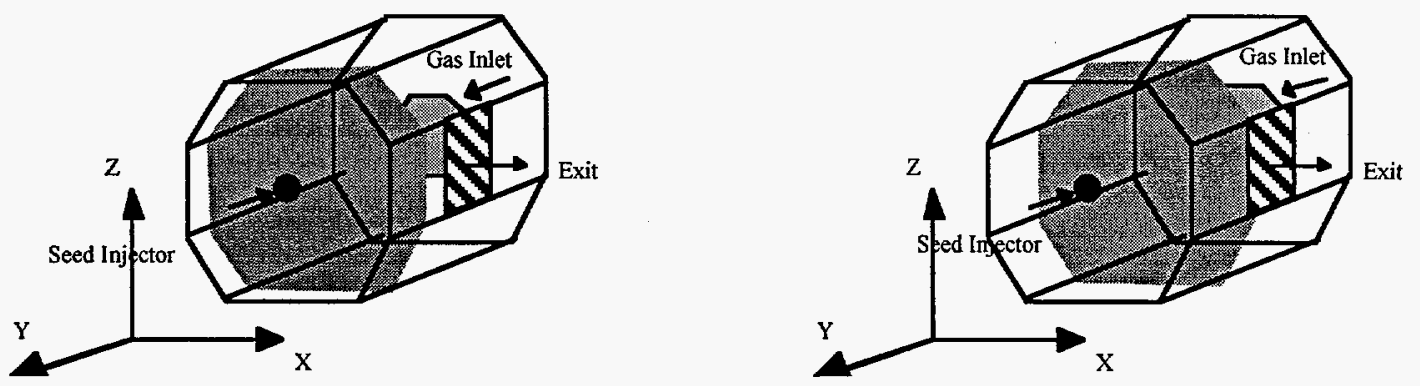

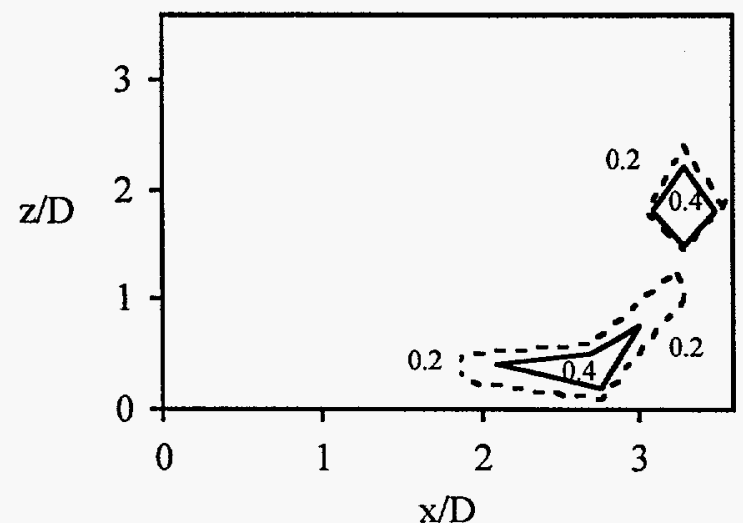

(a) $y / D=1.76$

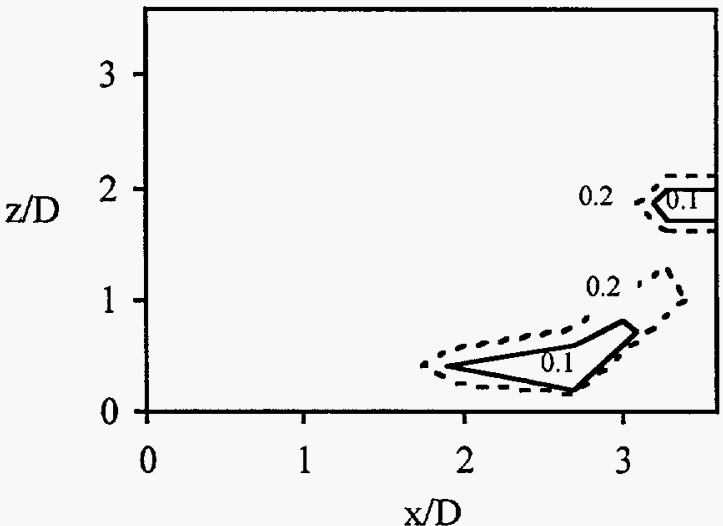

(b) $y / D=1.11$

Figure 9 Dispersion of Seed Particles in Various $x-z$ Planes

(Seed Injection at $\mathrm{x} / \mathrm{D}=1.85, \mathrm{y} / \mathrm{D}=2.21, \mathrm{z} / \mathrm{D}=1.85$ ) 


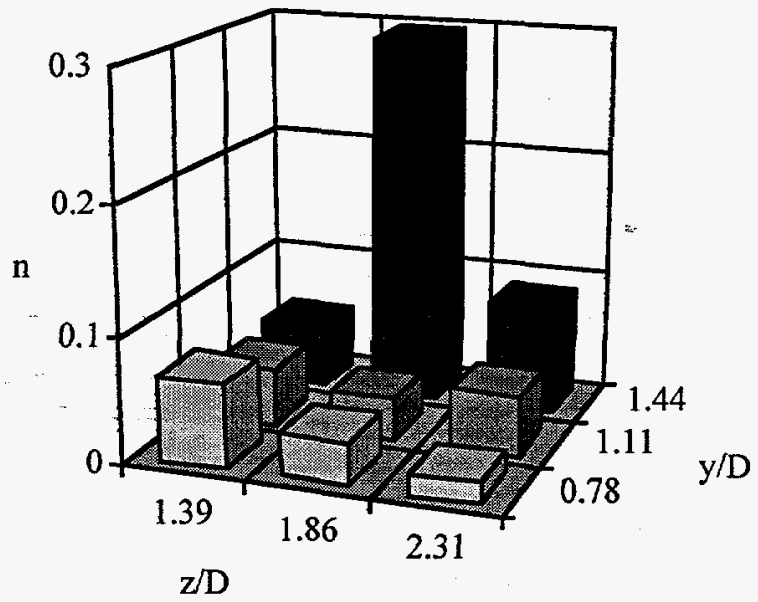

Figure 10 Distribution of Seed Particle Number Density, n, at the Exit (Seed Injection at $\mathrm{x} / \mathrm{D}=1.85, \mathrm{y} / \mathrm{D}=2.21, \mathrm{z} / \mathrm{D}=1.85$ )

Calculations of seed particle flow characteristics were also made for the intrusive-type seed injector and larger particle size. The injector is a cylinder with a $0.032-\mathrm{m}$ diameter, and it is mounted on the end plane with a $0.101-\mathrm{m}$ intrusion into the deswirl section as shown in Fig. 4c.

Figure 11 shows two dispersion patterns of seed particles in the deswirl section: (a) in an $x-z$ cross section in the mid plane, $y / D=0.5$, and $(b)$ in a $y-z$ cross section at $z / D=0.21$. In the figure, the shaded area represents the area where locally computed seed particle number density is higher than $0.1 \%$ of the injection value. In this calculation, the particle size is $400 \mu \mathrm{m}$ and the injection particle number density is $2.85 \times 10^{8} \mathrm{\#} / \mathrm{m}^{3}$. Figure 11 a shows that seed particles move toward the inlet plane and along the way some are turned to the right (or exit) side by the gas flow which moves in the general direction of the exit. There is a particle flow stagnation point (zero particle velocity) in center right next to the circular inlet. For seed particles larger than $400 \mu \mathrm{m}$, calculations became numerically unstable because particles move past the inlet plane into the first-stage combustor, which violates the inlet boundary conditions. This is consistent with the TRW report that seed material was found in the slag layer of the first-stage combustor in post test analyses. Figure $11 \mathrm{~b}$ shows that seed particles are driven from the center of the deswirl section where the seed is injected to the periphery by the gas flow. The inlet swirl flow spreads out the seed particles in a clockwise direction. Some particles leave the deswirl section through the exit and about $80 \%$ of the particles deposit in slag layer on the wall in the lower right corner, which is consistent with the discussion in the previous section. The seed in the slag layer may react with slag and form insoluble compound in amorphous phase or it may be vaporized and reentrained into the gas flow. The vaporization of the seed material which takes heat from the slag layer and the thickening of the deposition layer are the main reasons accounting for reduction of the heat loss from the deswirl section as reported by TRW [4]. 

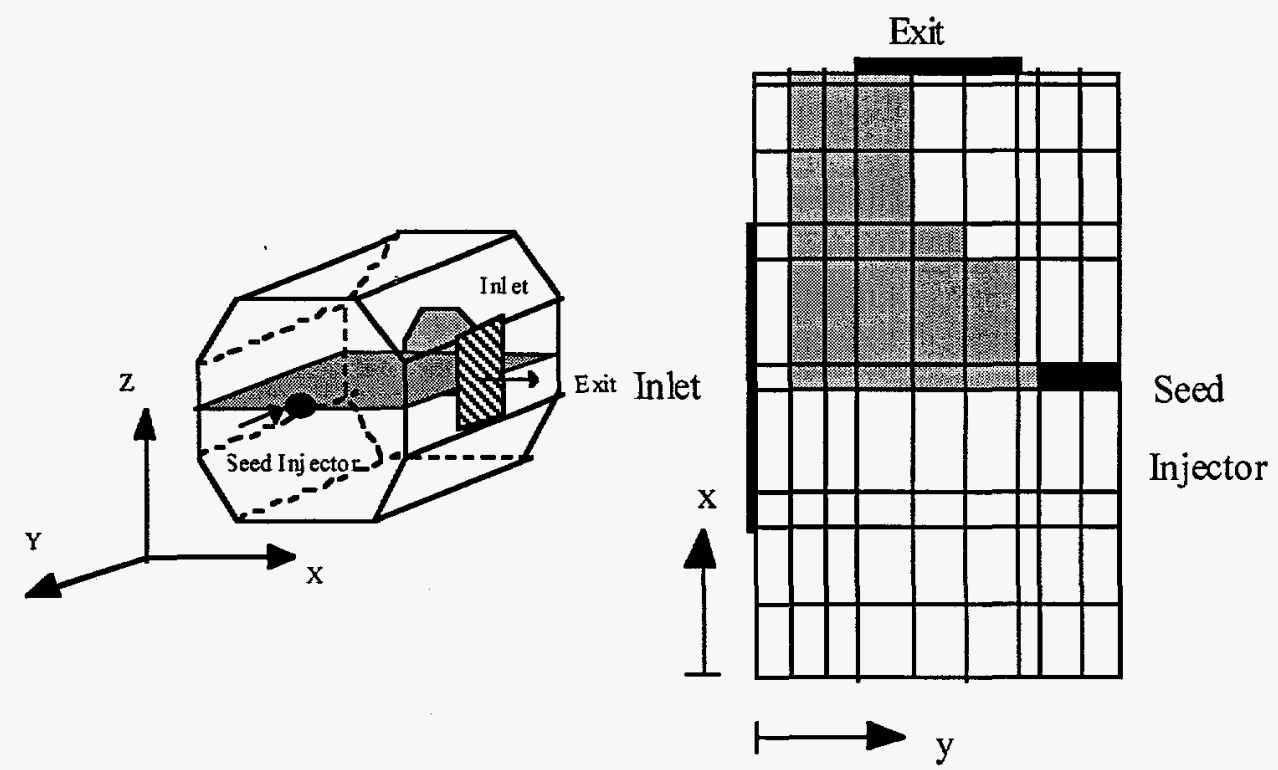

(a) $\mathrm{z} / \mathrm{D}=1.85$
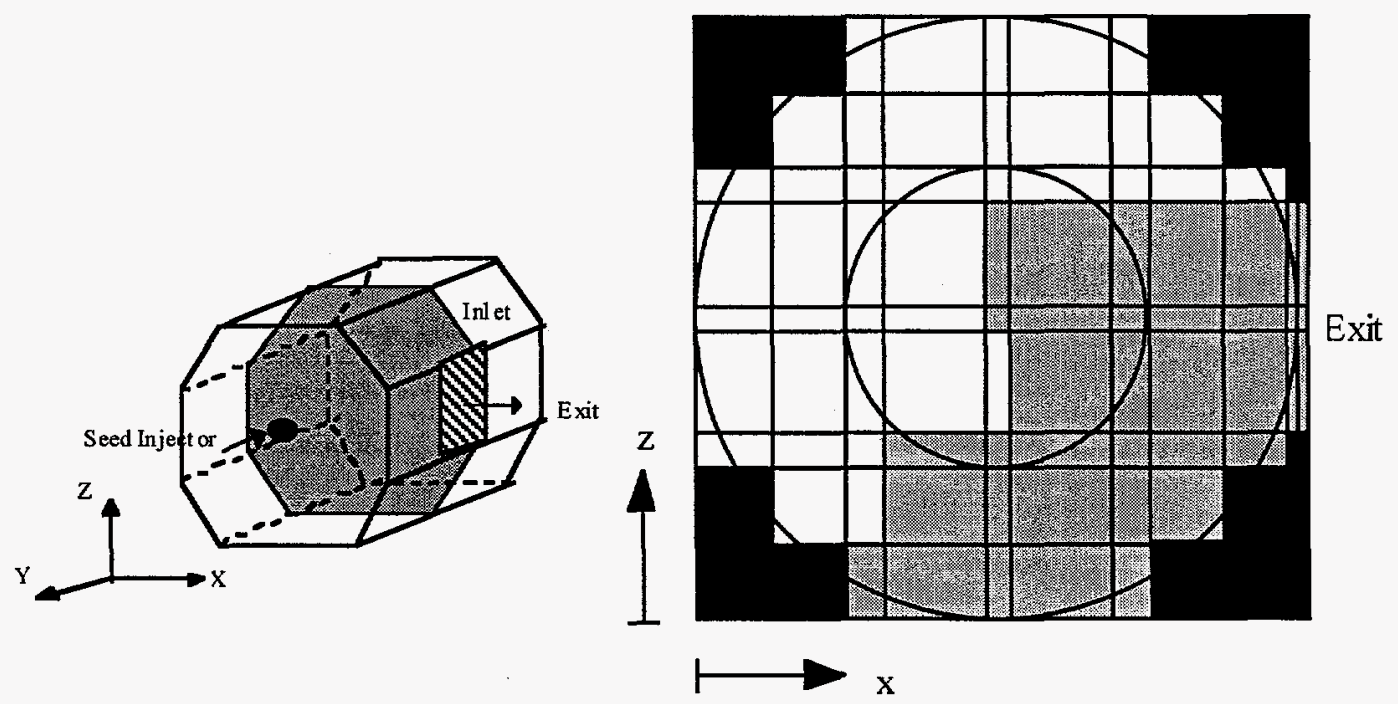

(b) $y / D=0.78$

Figure 11 Dispersion of Seed Particles by Intrusive Injection

(Seed Injection at $\mathrm{x} / \mathrm{D}=1.85, \mathrm{y} / \mathrm{D}=2.21, \mathrm{z} / \mathrm{D}=1.85$ )

Computational results show extremely asymmetric seed particle loading at the exit opening and a high percentage of seed particle deposition on the wall. Particle size, inlet swirl, injection velocity, and injection location are major factors affecting the asymmetric loading at the exit and wall deposition. The percentage of seed-particle deposition on the wall is estimated as $80 \%$ for $400-\mu \mathrm{m}$ particles. Table 3 shows the distribution of seed-particle loading at the exit for $400-\mu \mathrm{m}$ particles. The particle-number densities in the Table are normalized by the inlet value. 
The result clearly shows that seed particles at the exit concentrate in the right side (looking from the second-stage combustor), especially in the lower right-hand corner. The prediction is consistent with the TRW report that the excess particle accumulation was found in the lower right-hand corner of the second-stage combustor upstream of the oxygen injectors [4].

Table 3 Exit Seed Particle Loading $(400 \mu \mathrm{m})$

\begin{tabular}{|c|c|c|c|}
\hline \multirow{2}{*}{$\mathrm{y} / \mathrm{D}$} & \multicolumn{3}{|c|}{$\mathrm{z} / \mathrm{D}$} \\
\cline { 2 - 4 } & 1.39 & 1.86 & 2.31 \\
\hline 0.606 & 0.000 & 0.000 & 0.63 \\
\hline 0.500 & 0.000 & 0.016 & 0.68 \\
\hline 0.395 & 0.011 & 0.004 & 1.00 \\
\hline
\end{tabular}

Calculations were also made for two other seed particle sizes: 200 and $300 \mu \mathrm{m}$. Similarly, the percentages of seed particle deposition are high and seed particle loadings at the exit peak in the lower right-hand corner. However, the degree of asymmetry of the exit seed particle loading and the percentage of particle-wall deposition are reduced for a smaller particle size. The percentages of particle wall deposition are 50 and $65 \%$ for the particle sizes $200-$ and $300-\mu \mathrm{m}$, respectively.

\subsection{Optimal Seed Utilization in the Deswirl Section}

In an extensive parametric study, calculations were made for 37 injection locations on the end wall at $y / D=2.21$. The percentages of injected seed particles that deposit on the walls of the deswirl section were computed for these injection locations. Figure 12 shows the seed-particle deposition percentages according to the injection locations. The result clearly shows that the seed injection location has a decisive effect on the seed particle deposition percentage. The seed particle deposition percentage is highest (93\%) when the seed is injected from a location in the far left side of the deswirl section $(x / D=0.46$ and $z / D=1.85)$; the deposition percentage is $74 \%$ when the seed is injected from the center; and the deposition percentage is the lowest $(5.4 \%)$ when the seed is injected from the upper right corner $(\mathrm{x} / \mathrm{D}=3.47$ and $\mathrm{z} / \mathrm{D}=2.31)$.

When the seed is injected from the upper right corner $(x / D=3.47$ and $z / D=2.31)$, the seed-particle deposition percentage on the walls of the deswirl section is the lowest, at $5.4 \%$. Figure 13 shows contours of seed particle-number density in a $y-z$ cross section. The figure shows that seed particles move along with the gas flow from the injection location to the exit opening and need to turn only when they reach the exit. Therefore, a majority of the seed particles (94\%) pass through the exit opening with the gas. 


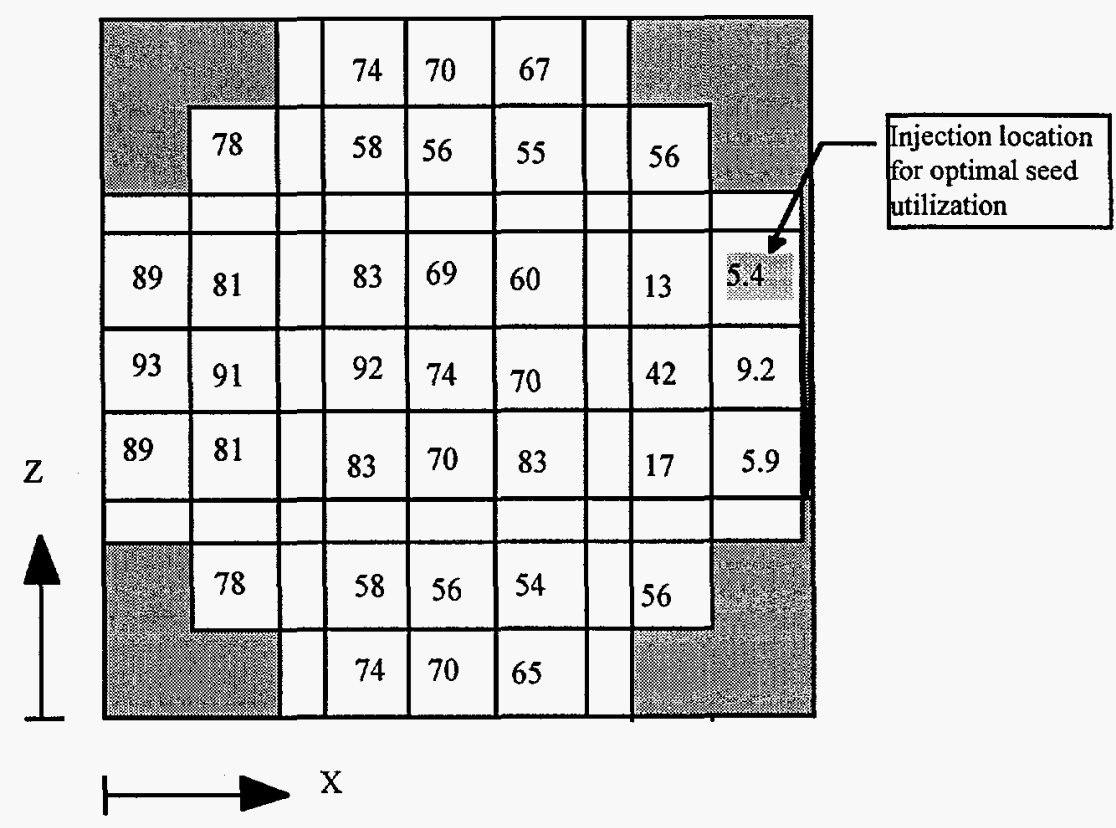

Figure 12 Percentages of Seed Particle Wall Deposition for Various Injection Locations
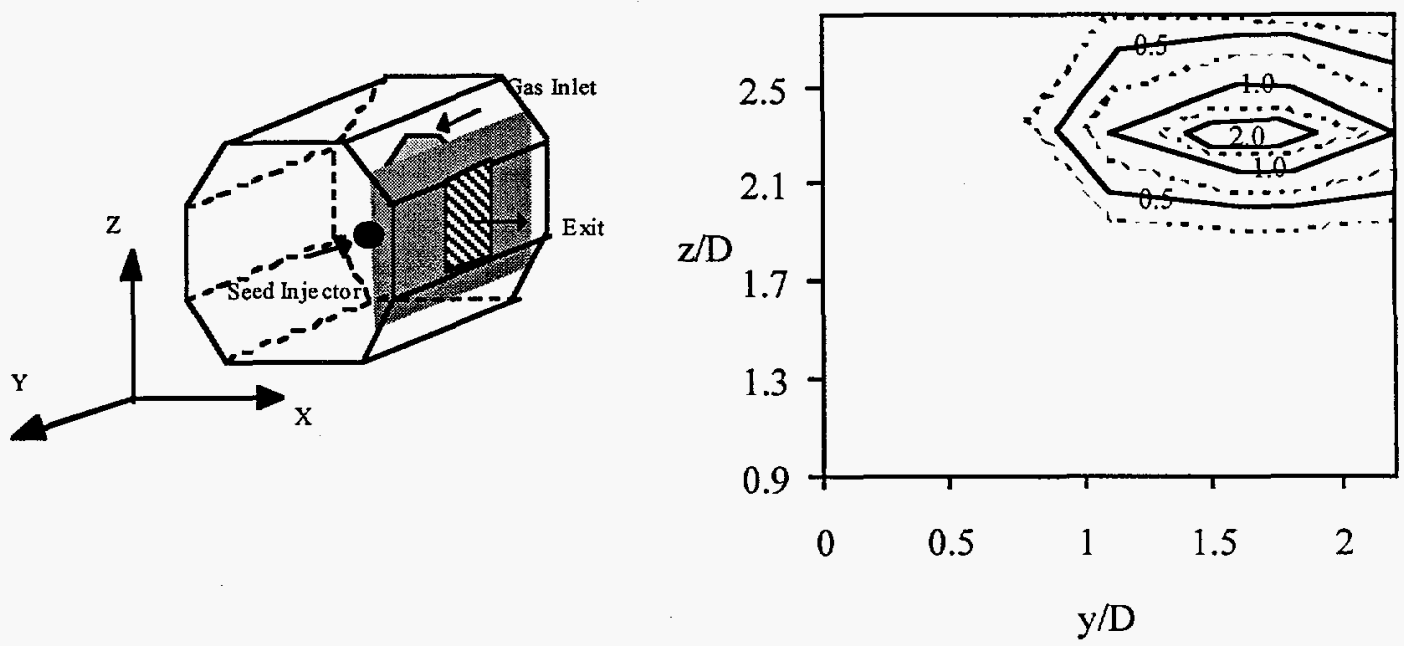

Figure 13 Seed Particle Number Densities at $x / D=3.47$

(Seed Injection at $\mathrm{x} / \mathrm{D}=3.47, \mathrm{y} / \mathrm{D}=2.21, \mathrm{z} / \mathrm{D}=2.31$ ) 


\section{ANALYSIS OF FLUID MIXING IN THE SECOND-STAGE COMBUSTOR}

Streams of oxidizer (pure oxygen) are injected into the second-stage combustor and burn with the coal gas to raise gas temperature to $3000 \mathrm{~K}$ and ionization to $8 \mathrm{mho} / \mathrm{m}$, while maintaining the heat loss at a $2 \%$ level. Preliminary tests showed that the mixing of jet fluid, the completeness of combustion, and the uniform dispersion of seed material are the critical factors to achieve the goals of a high exit temperature and high ionization with nearly uniform distribution. ANL used two-dimensional versions of the ICOMFLO computer code to investigate the effects of fluid mixing, completeness of combustion, and uniform seed dispersion on the performance of the second-stage combustor. The analysis of fluid mixing in the secondstage combustor is presented in this chapter. The effects of combustion completeness and seed dispersion are discussed in Chapters 4 and 5 , respectively.

\subsection{The Second-Stage Combustor}

A schematic of the MHD second-stage combustor between the deswirl section and the converging nozzle is shown in Fig. 14. The combustor is a channel consisting of an inlet plane (left), an exit plane (right), four side walls (top, bottom, front, and back), and oxidizer injectors on the top and bottom walls. The inlet stream includes coal gas and seed (potassium) particles. The coal gas is the product of substoichiometric coal-air combustion in a first-stage swirl combustor and seed particles are added to the coal-gas stream in the deswirl section. After entering the second-stage combustor, the coal-gas stream is mixed with the oxidizer injected from the top and bottom side walls. The mixture burns and produces high-temperature gas products. The high-temperature gas vaporizes seed particles and ionizes seed vapor. The plasma stream of ionized seed vapor and combustion products leaves the combustor through the exit. Figure 14 shows that the mid-plane (the shaded area) between front and back walls is selected as the computational domain for two-dimensional (2-D) flow simulation. For the 2-D flow simulation, oxidizer injection holes are replaced by two narrow openings (slots) on the top and the bottom walls, respectively. Three separate flow analyses were conducted for the secondstage combustor: jet fluid mixing, combustion (Chapter 4), and seed dispersion (Chapter 5). The inlet stream is coal gas and two jet streams are oxidizer. The two fluids mix in the combustor. The combustion analysis is presented in Chapter 4. The inlet stream consists of coal and inert gases and the two jet streams are oxidizer. Coal gas and oxidizer burn in the combustor. The analysis of seed dispersion is presented in Chapter 5. The inlet stream consists of coal gas, inert gas, and seed particles, and the two jet streams are oxidizer. Seed particles vaporize in the combustor.

Figure 15 shows the 2-D computational domain whose boundaries include an inlet (left dashed line), an exit (right dashed line), and two injector slots (arrows) on the top and the bottom side walls. An injection angle $\theta$ is defined as the angle between the downstream direction of the side wall and the bottom jet vector. 


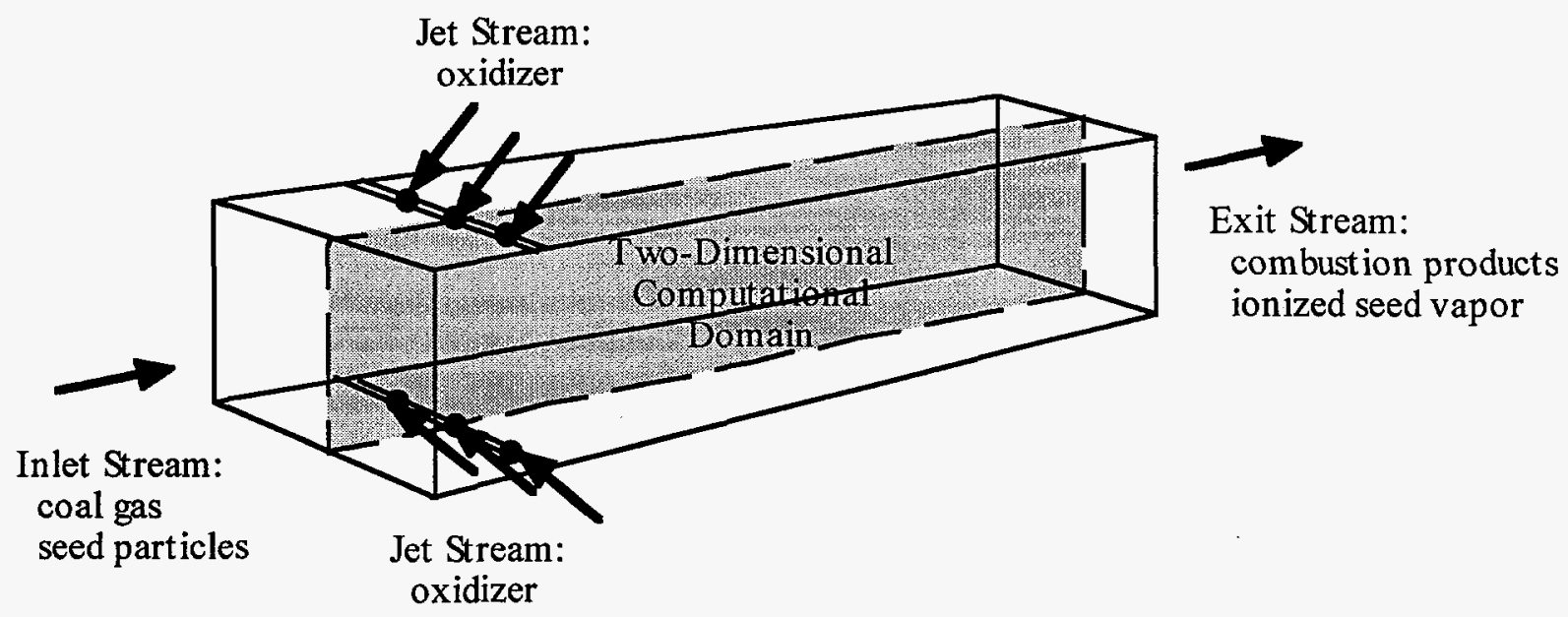

Figure 14 Schematic of the MHD Second-Stage Combustor

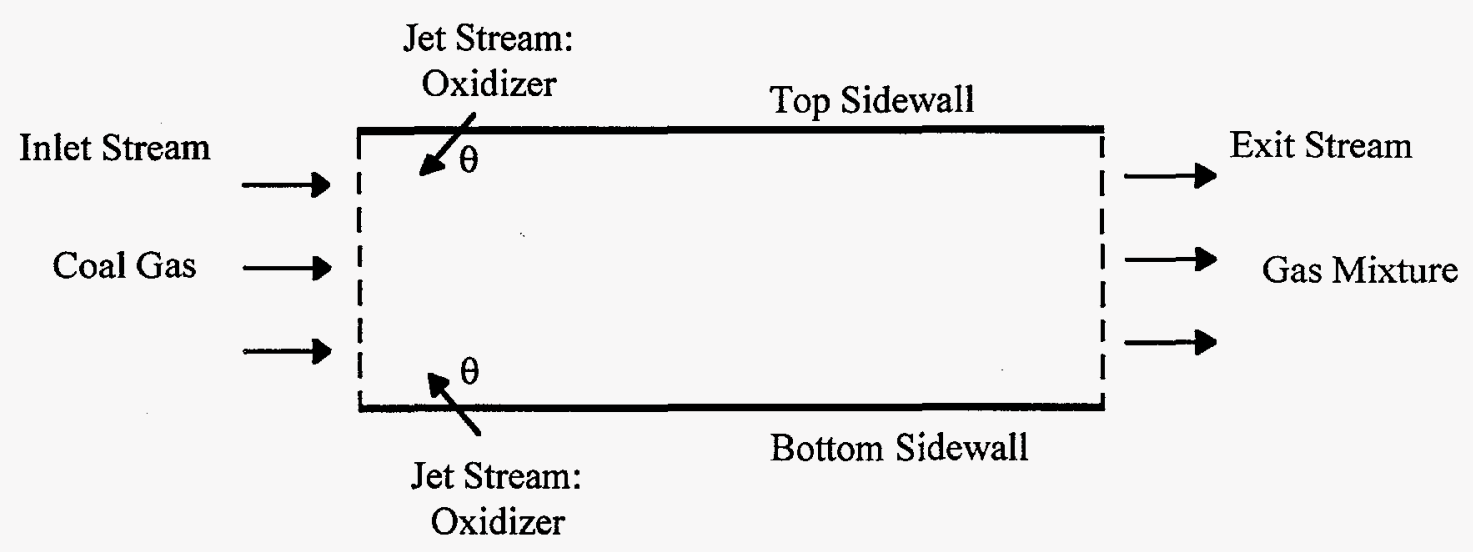

Figure 15 Computational Domain of 2-D Mixing Flow in the MHD Second-Stage Combustor

\subsection{Single-Phase 2-D Nonreacting Flow Computer Code}

The ICOMFLO computer code is described in detail in a separate ANL report entitled, The Multiphase Integral Reacting Flow Computer Code (ICOMFLO): User's Guide. A brief description of the ICOMFLO computer code for single-phase, 2-D, nonreacting flow is given in this section.

General conservation laws expressed by elliptic-type partial differential equations are used in conjunction with rate equations governing the mass, momentum, species, turbulent kinetic energy, and turbulent dissipation rate. A governing equation balances convective, diffusive, and source terms of a flow property. Convective terms are associated with velocity components and diffusive terms are associated with effective diffusivity of the flow property. For a nonreacting flow, there is no source term in the mass (or continuity) and species equations. The source terms 
of the two momentum equations include pressure gradient and shear stress terms. For convenience in numerical formulation, the governing equations are put in the following general form:

$$
\frac{\partial \mathrm{J}_{\mathrm{x}}}{\partial \mathrm{x}}+\frac{\partial \mathrm{J}_{\mathrm{y}}}{\partial \mathrm{y}}=\mathrm{S}_{\xi}
$$

where $\mathrm{J}_{\mathrm{x}}$, and $\mathrm{J}_{\mathrm{y}}$ are the combined convective and diffusive flux terms in the $\mathrm{x}$ and $\mathrm{y}$ directions and $S_{\xi}$ is the source term for a general flow property $\xi$. The general flow variable is the scalar 1 in the continuity equation, a velocity component $u$ or $v$ in a momentum equation, an enthalpy $h$ in the energy conservation equation, a species concentration in a species conservation equation, or a turbulent kinetic energy $\mathrm{k}$ or a turbulent dissipation rate $\varepsilon$ in one of the turbulence transport equations. The flux terms in Eq. 3.1 are expressed as

$$
\begin{aligned}
& J_{x}=\rho u \xi-\Gamma_{\xi} \frac{\partial \xi}{\partial x} \\
& J_{y}=\rho v \xi-\Gamma_{\xi} \frac{\partial \xi}{\partial y}
\end{aligned}
$$

where $\rho$ is density and $\Gamma$ is effective diffusivity. Effective diffusivity is the sum of laminar diffusivity and turbulent diffusivity. Laminar diffusivity is a fluid property that can be measured. For most fluids, laminar diffusivities are readily available in the literature. Turbulent diffusivity is a flow property that needs to be locally determined in a flow. The ICOMFLO computer code employs a two parameter $k-\varepsilon$ model to determine turbulent diffusivity $\mu_{t}$ in a flow field.

Launder and Spalding [13] assumed that the turbulent diffusivity $\mu_{\mathrm{t}}$ is proportional to gas density $\rho$, velocity fluctuation $u^{\prime}$ and a macro turbulent length scale $\lambda_{t}$ as shown in Eq. 3.4 , in which $\mathrm{C}_{\mu}$ is the proportionality constant.

$$
\mu_{t}=C_{\mu} \rho u^{\prime} \lambda_{t} / 2
$$

In Eq. 3.4, u' and $\lambda_{t}$ are two unknown flow properties to be determined. For an eddy size $\lambda_{t}$, the turbulent time scale is $\lambda_{t} / u^{\prime}$. Large eddies are assumed to lose their kinetic energy to small eddies through dissipation within one "turnover" time $\lambda_{t} / u^{\prime}$. Therefore, the rate of dissipation is equal to the transport rate of turbulent kinetic energy as shown in Eq. 3.5.

$$
\varepsilon=k\left(u^{\prime} / \lambda_{\mathrm{t}}\right)
$$

From Eq. 3.5, the macro turbulent length scale can be expressed as

$$
\lambda_{\mathrm{t}}=\mathrm{k}\left(\mathrm{u}^{\prime} / \varepsilon\right)
$$

Substituting Eq. 3.6 into Eq. 3.4, an alternative turbulent diffusivity expression is obtained as

$$
\mu_{\mathrm{t}}=\mathrm{C}_{\mu} \mathrm{\rho k}^{2} / \varepsilon
$$


In the turbulent diffusivity equation, Eq. 3.7, there are still two unknown flow properties, $\mathrm{k}$ and $\varepsilon$. Launder and Spalding proposed to solve for the two unknowns from two transport equations. Eqs. 3.8 and 3.9 are derived for the turbulence quantities $\mathrm{k}$ and $\varepsilon$, respectively. These equations are to be solved simultaneously with the governing equations of a single-phase flow. The source terms of the k-equation imply that the turbulent kinetic energy is generated by the viscous dissipation in the mean velocity field, while it is dissipated by turbulent fluctuations at a rate $\varepsilon$. The source term of the $\varepsilon$-equation is similar to the two terms in the k-equation except they are multiplied by a factor $(\varepsilon / \mathrm{k})$ and by the empirical constants $\mathrm{C}_{1}$ and $\mathrm{C}_{2}$.

Turbulence transport equation of $\mathrm{k}$ (single phase)

$$
\frac{\partial}{\partial x}\left(\rho u k-\frac{\mu_{t}}{\sigma_{k}} \frac{\partial k}{\partial x}\right)+\frac{\partial}{\partial y}\left(\rho v k-\frac{\mu_{t}}{\sigma_{k}} \frac{\partial k}{\partial y}\right)=\mu_{t} G_{\mu}-\rho \varepsilon
$$

Turbulence transport equation of $\varepsilon$ (single phase)

$$
\frac{\partial}{\partial x}\left(\rho u \varepsilon-\frac{\mu_{t}}{\sigma_{\varepsilon}} \frac{\partial \varepsilon}{\partial x}\right)+\frac{\partial}{\partial y}\left(\rho v \varepsilon-\frac{\mu_{t}}{\sigma_{\varepsilon}} \frac{\partial \varepsilon}{\partial y}\right)=C_{1} \mu_{t} \frac{\varepsilon}{k} G_{\mu}-C_{2} \rho \frac{\varepsilon^{2}}{k}
$$

Equations 3.7, 3.8, and 3.9 constitute a turbulence model for a single-phase flow. In this model, there are five empirical constants, $\mathrm{C}_{\mu}, \mathrm{C}_{1}, \mathrm{C}_{2}, \sigma_{\mathrm{k}}$, and $\sigma_{\varepsilon}$. The values of these empirical constants, recommended by Launder and Spalding [13], are listed below.

$\begin{array}{cr}\mathrm{C}_{\mu} & 0.09 \\ \mathrm{C}_{1} & 1.44 \\ \mathrm{C}_{2} & 1.92 \\ \sigma_{\mathrm{k}} & 1.0 \\ \sigma_{\varepsilon} & 1.3\end{array}$

The gas mixture is assumed to behave like a perfect gas, obeying the following equation of state:

$$
\mathrm{pW}=\rho \mathrm{RT}
$$

where $\mathrm{W}$ is molecular weight of the mixture.

A staggered and variably spaced grid system is used for the numerical calculation, with the gas-velocity components stored on the cell surfaces and all other physical quantities stored at the nodal points of each cell (or scalar cell). The governing partial-differential equations are transformed into algebraic equations by integrating over a computational cell. These algebraic equations are solved using a Semi-Implicit Method for Pressure-Linked Equations-Revised (SIMPLER) algorithm originally developed by Patankar and Spalding [12]. A line-by-line sweep is used in the primary flow direction to solve the system of algebraic equations. 


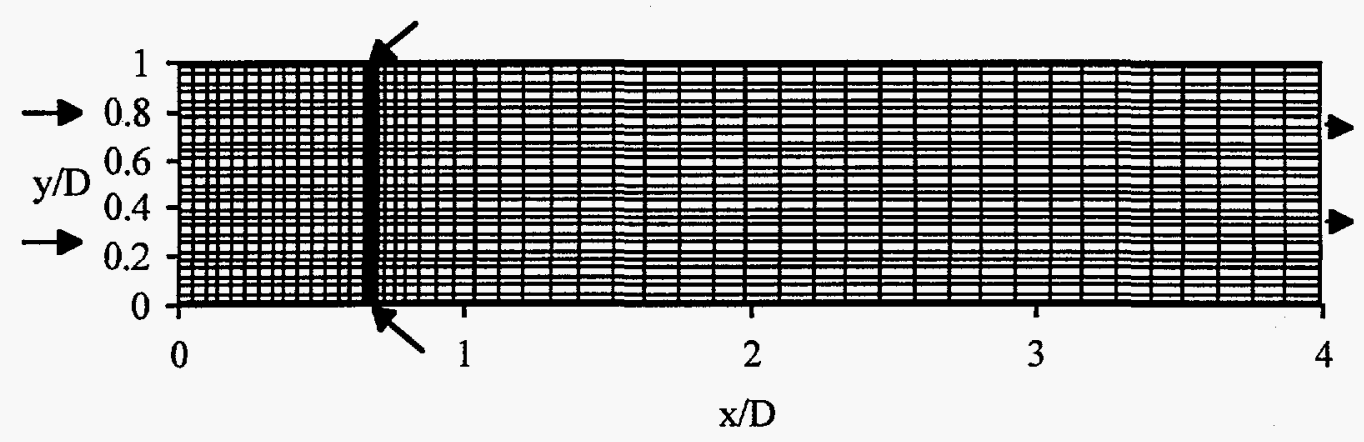

Figure 16 A 53 x 32 Grid System for the MHD Second-Stage Combustor

Figure 16 shows a grid system of $53 \times 32$ interior nodes for the 2-D computational domain. The coordinate system is rectangular, with an origin at the lower left corner, a horizontal $x$-axis, and a vertical y-axis. Evenly spaced grid points are used for the y-axis, except for the cells adjacent to the wall, which must be wide enough to bridge the near-wall viscous layer, allowing the application of wall functions. Variably spaced grid points are defined for the $\mathrm{x}$-axis to better resolve the flow field in the vicinity of the jet slots. A denser packing of grid points is defined near the jet slots where large flow property gradients occur. The grid system was selected based on a sensitivity study of the effects of grid refinement on computational results for the system being studied. The grid selected represents a good balance between the goals of insensitivity to further refinement and computational cost.

Values are assigned to flow variables at the inlet plane and jet slots in the side walls. A reference pressure is assigned at the midpoint of the inlet plane. This assignment is necessary to determine absolute pressure over the flow domain for the computation of density from the equation of state; only relative pressure is determined from solution of the conservation equations. Except at the jet slots, the side walls are impermeable and therefore the no-slip conditions apply at the wall. A momentum wall function is used to bridge the near-wall boundary layer. When the jet velocity is specified at the jet inlet, the compressibility of jet inlet conditions affects the mass flow rate of the jet because pressure is no longer a free boundary condition, but rather needs to be determined from the flow solution in the interior. A procedure added to the computer code dynamically alters the computational grid to adjust the area of the jet during iteration toward the solution in such a way that the jet mass flow rate computed matches an input value [14]. Patankar's [12] locally one-way flow assumption is applied to the outflow boundary, eliminating the need to specify the values of flow variables at the outflow boundary. In this outflow boundary formulation, the stream-wise diffusion coefficients are taken to be zero at the outflow boundary.

To study the effects of asymmetric main flow inlet velocity profiles on mixing with jet injection, the computational grid covers a full-channel depth. A previously employed alternating direction, line-by-line sweep solution method [12] was observed not to preserve symmetry in the solution when symmetrical inlet profiles were employed as boundary conditions and the initial 
guess of flow field variables was symmetrical. Numerical experiments and analysis of the methodology revealed that line-by-line sweeps across the plane of symmetry destroyed symmetry in the intermediate results, and that the asymmetry introduced became self preserving in the iterative procedure in cases of strong jet injection from the side walls. Symmetry is achieved in the solution, however, when the line-by-line sweep is performed only in the primary flow direction. This change in the computer code was made to provide a smooth transition between symmetrical and asymmetric cases and to help ensure that asymmetries in the computed solutions are a physical consequence of asymmetric boundary conditions.

The SIMPLER algorithm used in the ICOMFLO code solves the governing equations in a staggered grid system, numerically and iteratively. In principle, the iteration routine, if it converges, should yield a solution which satisfies all governing equations simultaneously to an arbitrary degree of accuracy for a sufficiently refined grid. However, the discrete nature of the computation in a digital computer, as well as approximations made in the numerical scheme (e.g., dropping of higher-order terms of small numbers, etc.), make reduction of numerical error in the solution below a certain level through continued iteration impossible to achieve. Instead, a cutoff point for the iteration scheme is defined, so that calculations do not proceed unnecessarily when the iteration has reached a level of acceptable accuracy. At this level, the computation is considered to be converged, and the conditions which must be satisfied to make this determination are the convergence criteria.

The convergence criterion used in the ICOMFLO code is based primarily on the computed mass residual. In each iteration cycle, velocities obtained from solving the momentum equations no longer satisfy the continuity equation in an unconverged solution. The mass residual is calculated from the velocities obtained by solving the momentum equations by summing mass fluxes across each face of each cell in the computational domain. This sum should be zero for all points in the domain when the continuity equation is satisfied by the current pressure, velocity, and density fields simultaneously. In a typical computation, the maximum mass residual is compared to the convergence criteria after each iteration over the system of governing equations. When the mass residual becomes smaller than the convergence criteria, the solution is considered converged and the iteration routine completes processing and outputs results.

Calculations were performed on ANL's CRAY/X-MP supercomputer to predict the mixing patterns of the oxidizer jet and the inlet coal-gas streams in the MHD second-stage combustor. For a typical calculation, the maximum mass residual decreases to $10^{-5}$ in 50 iterations and to $10^{-15}$ in slightly more than 1000 iterations. After that, the floating point precision of the CRAY supercomputer prevents any further improvements in the convergence. Each iteration takes approximately one second of computing time. The choice of the preset convergence criterion is driven by the desire to see no further development of the computed flow field if iteration were to continue. No discernible difference exists in plotted results when iteration is terminated at a residual of $10^{-9}$ or is allowed to continue to a residual of $10^{-15}$. Given the negligible difference between the solutions iterated to a mass residual of $10^{-9}$ and $10^{-15}$, the additional computer time spent in pushing the residual closer to zero is unnecessary. Therefore, a convergence criterion of 
maximum mass residual of $10^{-9}$ was chosen for parametric studies. A typical computational run needs about 300 seconds of supercomputing time to meet the convergence criterion.

Two fluids (jet and coal gas) are mixed in the MHD second-stage combustor. The crosssectional average of the jet concentration $\mathrm{C}_{\mathrm{av}}$ and its normalized standard deviation $\sigma_{\mathrm{c}}$ are used to show the degree of fluid mixing on a cross-sectional area $A . C_{a v}$ and $\sigma_{c}$ are defined as follows:

$$
\begin{aligned}
& C_{a v}(x)=\frac{1}{A} \iint C d A \\
& \sigma_{c}(x)=\frac{1}{C_{a v}}\left[\frac{1}{A} \iint\left(C-C_{a v}\right)^{2} d A\right]^{1 / 2}
\end{aligned}
$$

$\sigma_{c}$ is referred to as the stratification factor in the following discussion. This factor is equal to zero if the two gases are homogeneously mixed. In a completely stratified flow, it is equal to one if the jet velocity is the same as the main velocity and higher than one if the jet velocity is faster than the main velocity, and lower than one if the jet velocity is slower than the main velocity. In general, the stratification factor monotonically decreases downstream of the plane of jet injection as the mixing process proceeds along the main flow direction.

\subsection{Effects of Operating Conditions on Fluid Mixing}

An initial step in performing detailed CFD analysis of the second-stage combustor of the MHD power train was to set up the computer code to compute using a rectangular coordinate geometry rather than a cylindrical coordinate geometry (the CFD code is capable of doing either), and to benchmark-test it using an analytically known laminar flow solution corresponding to the geometry of the second-stage combustor without any jet injection; a baseline case for nonreacting flow was then set up. This work proceeded in parallel with nonreacting flow jet injection experimental studies on the Plexiglas mock up of the second-stage combustor at TRW $[15,16]$. The baseline-case conditions chosen correspond to those frequently employed in the TRW experimental studies and provide a fixed basis for Argonne's CFD parametric studies of jet mixing in the second-stage combustor under isothermal conditions without reaction. Parametric studies were conducted to investigate the effects of jet angle, jet momentum, and inlet gas-flow symmetry on the mixing pattern of the jet and the inlet gas flows $[13,17-20]$.

The simulation conditions for the two-dimensional baseline calculations are listed in Table 4. These conditions are the ones used in the parametric sweeps discussed in the following sections, except for the variation of the particular parameter under study.

The results of the baseline calculations are displayed in Fig. 17. Significant-sized vortices can be seen downstream of the injectors, extending to $x / D=2.0$. Figure $17 \mathrm{c}$ shows contours of the $\log$ of the turbulence macro length scale or eddy size calculated from Eq. 3.6. These 
logarithms are all negative, so the larger the absolute value of the contour label in Fig. $17 \mathrm{c}$, the smaller the eddy size. In the recirculation zones, turbulent eddy size is noticeably smaller, indicating greater dissipation of turbulent kinetic energy. Figure $17 \mathrm{~b}$ shows jet mass concentration contours. Concentration gradients are clearly high in the vicinity of the jets and decrease rapidly in the downstream as flow sweeps past the recirculation zones. Although not shown in Fig. $17 \mathrm{~b}$, jet mass concentration is 1.0 at the jet inlet. The highest concentration contour of significant extent is that for a jet mass concentration of 0.5 , which extends only a short distance downstream of the jet injectors. This picture, therefore, indicates that a large amount of mixing occurs in the near vicinity of the jet injectors for this case. By the time the flow reaches the exit plane, the jet fluid is fairly well mixed, which is indicated by a low density of concentration contours in that region.

\section{Table 4 Baseline Conditions of Nonreacting Flow in the MHD Second-Stage Combustor}

\begin{tabular}{|l|c|}
\hline Bulk Jet Concentration & 0.1556 \\
\hline Inlet Reynolds Number & $1.1 \times 10^{5}$ \\
\hline Jet Velocity $\left(\mathrm{V}_{\mathrm{i}} / \mathrm{U}_{0}\right)$ & 13.7 \\
\hline Jet Angle (degree) & 130 \\
\hline Pressure (atm) & 1 \\
\hline Combustor Aspect Ratio (L/D) & 3.84 \\
\hline
\end{tabular}

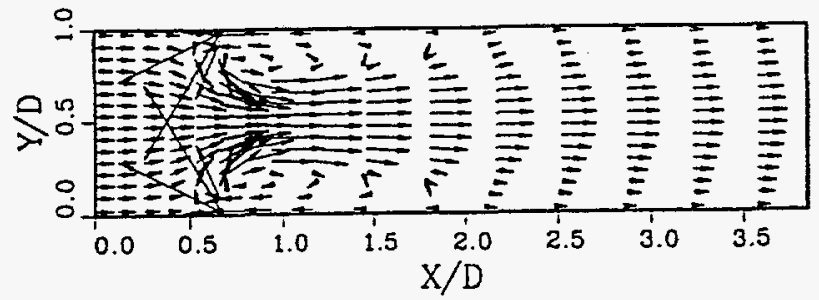

(a) Velocity Vectors

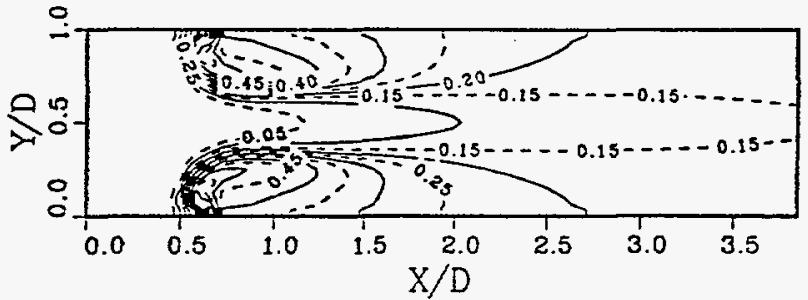

(b) Jet Mass Concentration

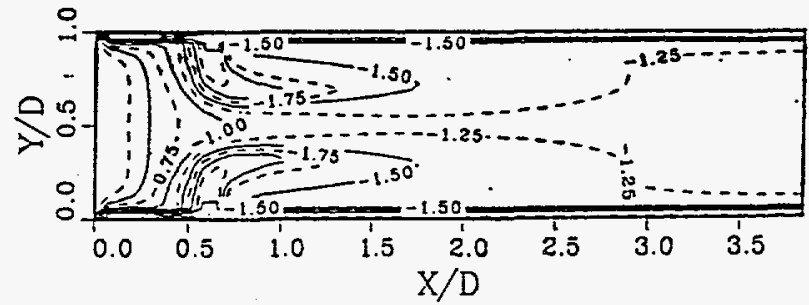

(c) Eddy Size

Figure 17 Jet Fluid Mixing Patterns and Turbulent Eddy Size - Baseline Case 


\subsubsection{Effects of Jet Angle}

The jet angle is defined as the angle between the jet velocity vector and the positive $\mathrm{x}$-axis. Computer runs for jet angles ranging from 50 to 150 degrees were performed to simulate the mixing pattern of gas and jet flows in an MHD second-stage combustor. The flow conditions for these runs were the same as the baseline flow conditions, except for the jet angle. When the jet has a velocity component in the positive $\mathrm{x}$-direction (jet angles less than 90 degrees) the jet configuration is called co-flow injection; when the jet has a velocity component in the negative $\mathrm{x}$-direction (jet angles greater than 90 degrees) the jet configuration is called counterflow injection. The co-flow and counterflow jet configurations were found to produce markedly different flow and mixing patterns in the interior of the duct.

The computed flow and mixing patterns for the two of 50-, 70-, 90-, and 130-degree jet angles are shown in Figs. 18 and 19, respectively. For each case, Fig. 18 shows velocity vectors and Fig. 19 shows contours of jet concentration.

For the 50-degree jet angle (co-flow injection) case, jet penetration is shallow, and consequently mixing over the chamber length is poor. In the region from the jet opening to the exit, jet flows are pushed toward the walls by the main stream gas flow. Consequently, flow velocity is higher in the outer regions and lower in the central region, and the jets are confined in a thick layer near the walls, as shown in Figs. 18a and 19a. After the jets have been turned downstream by the main flow, the shear layer between the high-speed jet flow and the low-speed gas flow is responsible for the further mixing of the two flows by turbulent and viscous momentum transport. Because the chamber length downstream of the jet ports is less than four chamber widths, the shear layer does not fully develop in the combustor. This incomplete development can be seen in Fig. 18a where the chamber center velocity is still low near the exit plane. In this case the velocity humps caused by the jets have not diffused to the center by the time the flow reaches the exit. This lack of development and shallow initial penetration of the jets leads to overall poor mixing. The $5 \%$ jet concentration contour does not reach the center line of the combustor for the case of a 50-degree jet.

The 70- and 90-degree jet angle cases in Figs. 18b, 18c, 19b, and 19c show intermediate states between the very different flow patterns of the 50- and 130-degree jet angle cases. At 70 degrees, small recirculation zones appear just downstream of the jets, but the jets are still turned rapidly by the main flow, keeping both the jet momentum and mass from penetrating effectively to the centerline. In the 70-degree jet angle case, the $5 \%$ jet concentration contour still does not reach the centerline before the chamber exit (Fig. 19b). For the 90-degree jet angle case, the recirculation zones downstream of the jets have grown, jet penetration into the main stream is much deeper, causing the flow to transition to a velocity profile with the velocity maximum in the chamber center by the time the chamber exit is reached (Fig. 18c), and mixing is much improved, with the $5 \%$ jet concentration contour reaching the chamber centerline well before the chamber exit at about $\mathrm{x} / \mathrm{D}=2.5$ (Fig. 19c). 


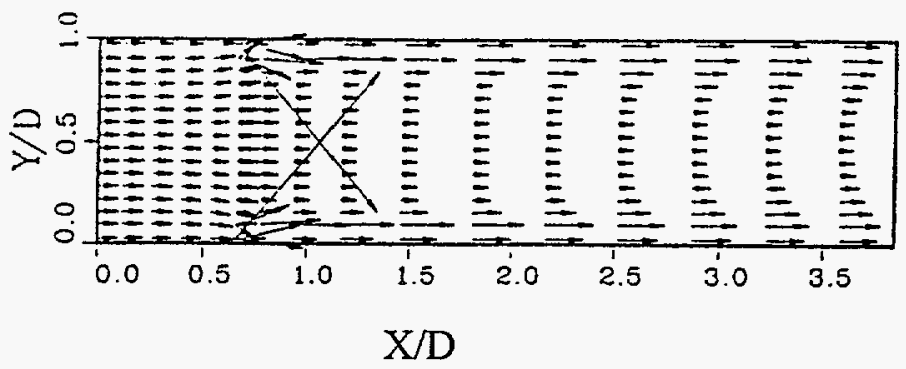

(a) Jet angle $=50$ degrees

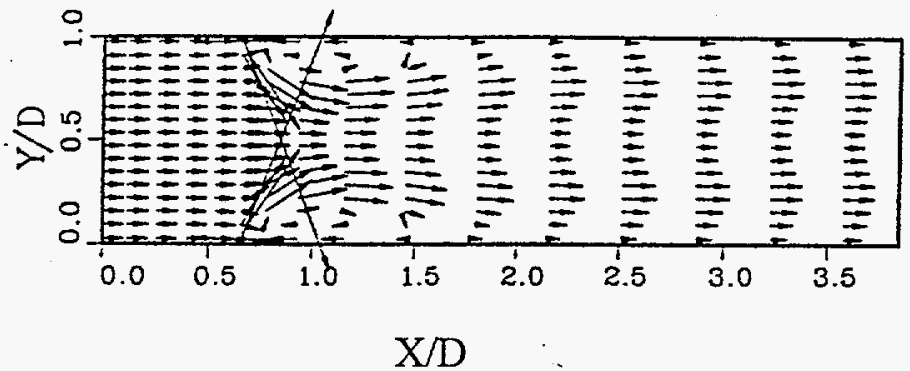

(b) Jet angle $=70$ degrees

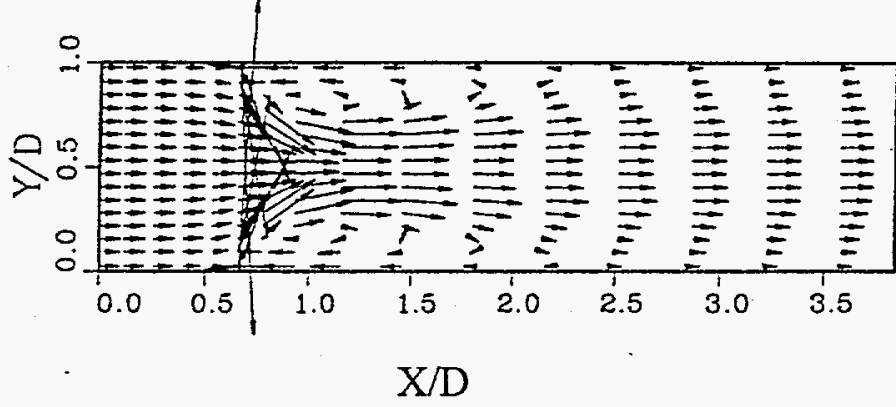

(c) Jet angle $=90$ degrees

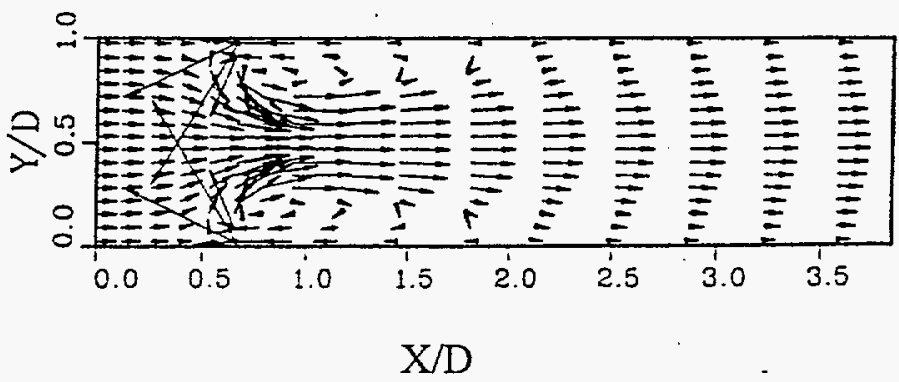

(d) Jet angle $=130$ degrees 


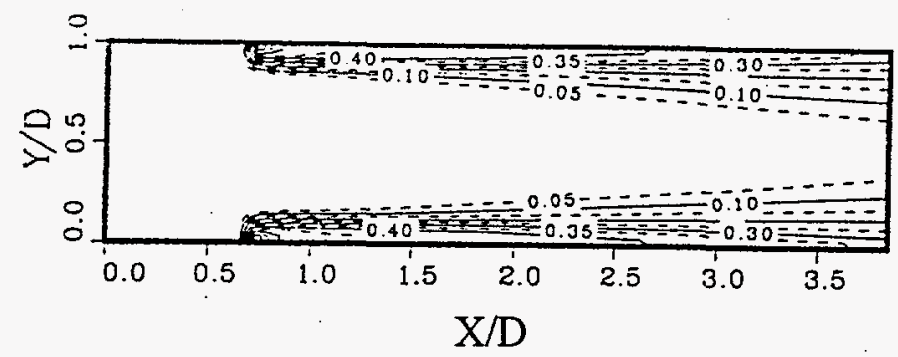

(a) Jet angle $=50$ degrees

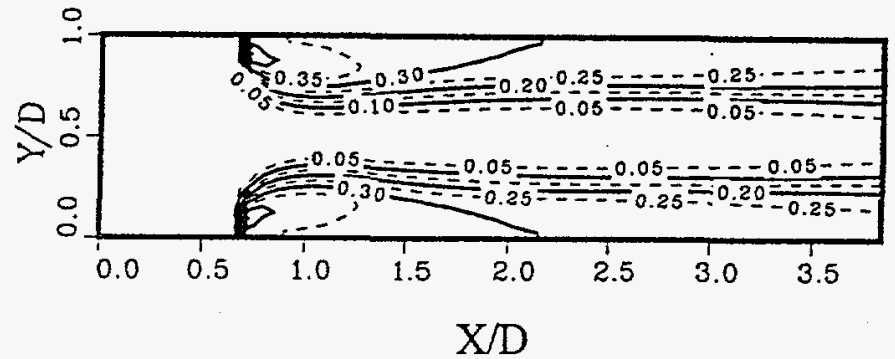

(b) Jet angle $=70$ degrees

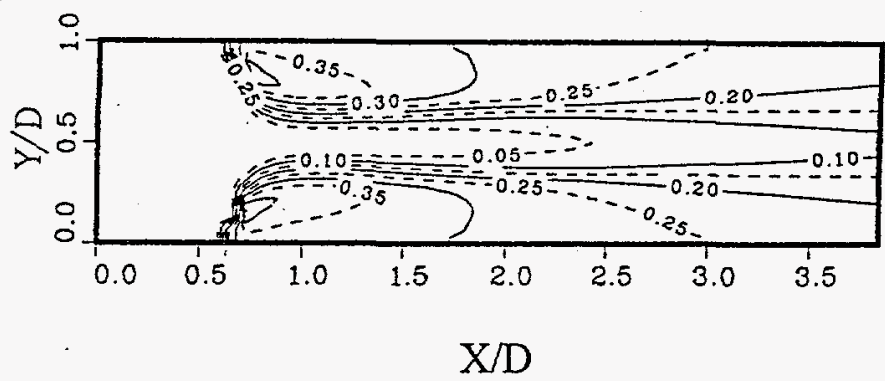

(c) Jet angle $=90$ degrees

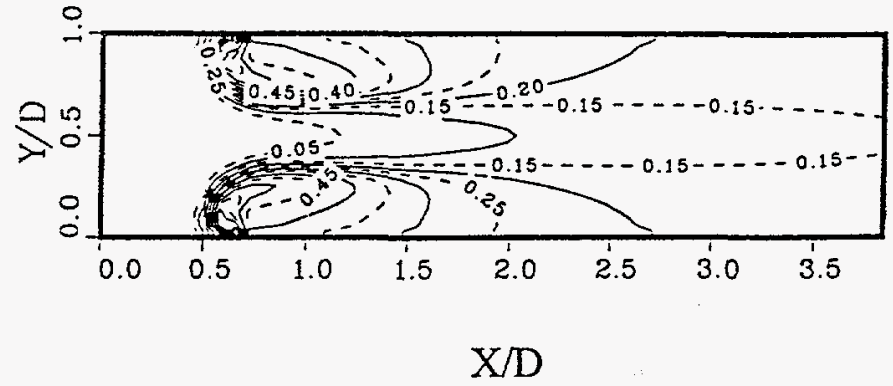

(d) Jet angle $=130$ degrees

Figure 19 Jet Fluid Concentration of Nonreacting Flow for Various Injection Angles 
For the 130-degree jet angle (counterflow injection) case, the jet penetration is much deeper and mixing is much better when compared with the co-flow injection cases. Convective jet penetration is the dominant process responsible for jet/main flow mixing. Large vortices are found extending from upstream of the jet ports, due to the counterflow injection to well downstream of the jet ports as shown in Fig. 18d. The 5\% jet concentration contour reaches the center line of the combustor at about $\mathrm{x} / \mathrm{D}=1.2$ as shown in Fig. 19d, much farther from the exit plane than in the other cases. In Fig. 19d, the exit axial velocity profile is highest in the middle. This high center velocity is not caused by wall viscous effects through characteristic confined flow development, but rather the high center velocity at the exit is a consequence of deeper jet penetration that forces the main flow to accelerate through the center of the chamber to move past the jets.

As defined in Section 2.2.4, the normalized standard deviation of jet mass concentration over a chamber cross section was chosen as a measure of mixedness, which decreases to zero as the main flow and jet fluids become completely mixed. Figure 20 shows the axial development of the jet/main flow mixing for four cases (50-, 70-, 90-, and 130-degree jet angles) using the jet concentration standard deviation as a measure of mixedness. Figure 20a plots the mean jet mass concentration versus the displacement along combustor length (or x-axis) and Fig. $20 \mathrm{~b}$ plots the normalized jet concentration standard deviation versus this displacement. A reference jet concentration is defined as the jet concentration of a uniform mixture of jet and gas. The reference jet concentration is 0.155 .

For the 50-degree jet angle case, mean jet concentration increases along the $\mathrm{x}$-axis and reaches about 0.14 at the exit, as shown in Fig. 20a. The exit mean jet concentration is lower than the reference jet concentration because the high jet concentration zones match the high velocity zones, due to poor initial jet penetration, as shown in Figs. 18a and 19a. In Fig. 20b, the normalized jet concentration standard deviation for 50-degree injection decreases from about a value exceeding 2.5 near the injection location to about 0.8 at the exit. The steep slope of the standard deviation curves near the jet ports is a consequence of rapid convective mixing due to jet penetration into the cross stream. This convective mixing is followed by a much slower diffusive mixing zone after the jets are turned by cross flow into the downstream (positive $\mathrm{X}$ ) direction.

For angles of 70 and above, the mean jet concentration has a maximum value near $\mathrm{x} / \mathrm{D}=1$, as shown in Fig. 20a. The exit mean jet concentration is higher than 0.155 , indicating lower jet concentration in higher velocity zones. The normalized jet concentration standard deviation at the exit plane decreases as injection angle is increased. With better jet penetration (for jet angles of 70 and above) the mixing is much more complete at the end of the convective mixing zone than in the 50-degree jet angle case.

For the 130-degree jet angle case, the jet has a large velocity component counter to the main flow direction at the injector location. The injected jet mass penetrates the upstream, is turned, and brought back downstream by interaction with the main flow. This flow configuration 
produces the sharp peak in mean jet concentration just upstream of the injector location (Fig. 20a). The cross-section mean concentration peak occurs because a large amount of jet fluid is flowing in both directions in that region while the total cross-section jet mass flow rate with downstream taken as positive must remain constant and equal to the mass flow rate through the injectors. Much of the mixing in this case occurs upstream of the injector plane. Figure $20 \mathrm{~b}$ shows that the normalized standard deviation of jet mass concentration is about 0.7 at the injection plane for 130-degree injection; the value for the other cases is much greater.

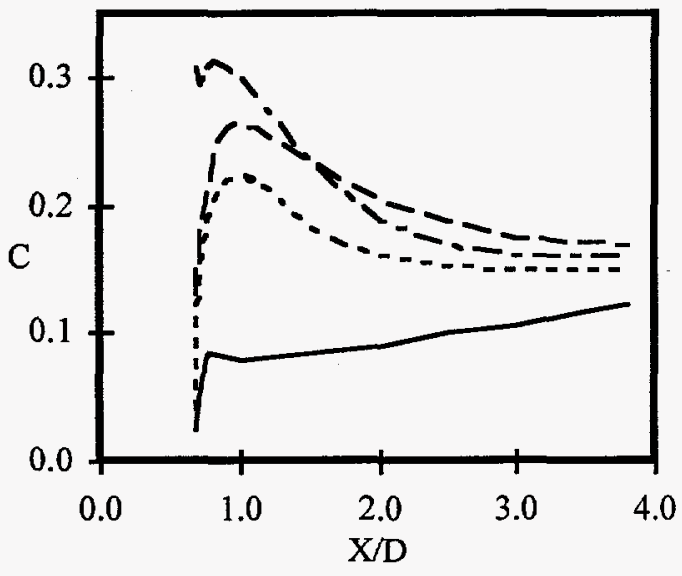

(a) Mean Concentration

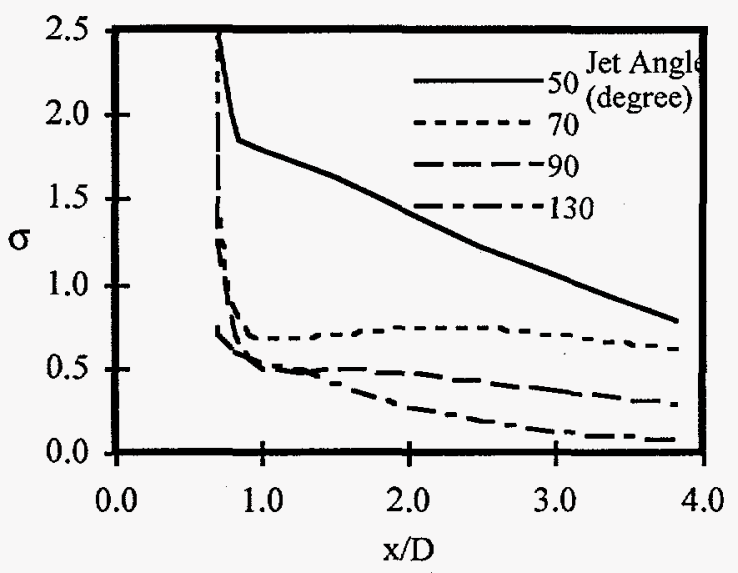

(b) Normalized Deviation

Figure 20 Effect of Injection Angle on Jet Fluid Mixedness in the Combustor

For comparison, the exit velocity and jet concentration of these four cases are plotted in Fig. 21. The exit velocity of 50- and 70-degree cases has double humps near the walls and the exit velocities of the other two cases have their maximum velocities at center, as shown in Fig. 21a. The exit jet concentration profiles become progressively more uniform as the jet injection angle is increased (Fig. 21b), and this increasing uniformity means the jet fluid is more completely mixed with the fluid of the main flow.

Results of a parametric sweep over jet angles are shown in Fig. 22. The normalized standard deviation of jet concentration at $\mathrm{x} / \mathrm{D}=3.8$ versus jet angle is plotted in this figure. Several characteristics of the mixing dependence on injection jet angle are apparent in Fig. 22. Jet mixing is better for jet angles larger than 90 degrees. Jet/main flow mixing depends strongly on jet injection angle for angles in the range 40 to 90 degrees, and the mixing rate increases with increasing injection angle. Above 90 degrees, the mixing rate is not as strongly dependent on injection angle. Counterflow injection angles in the range 130 to 140 degrees appear to be near the optimum for mixing. 


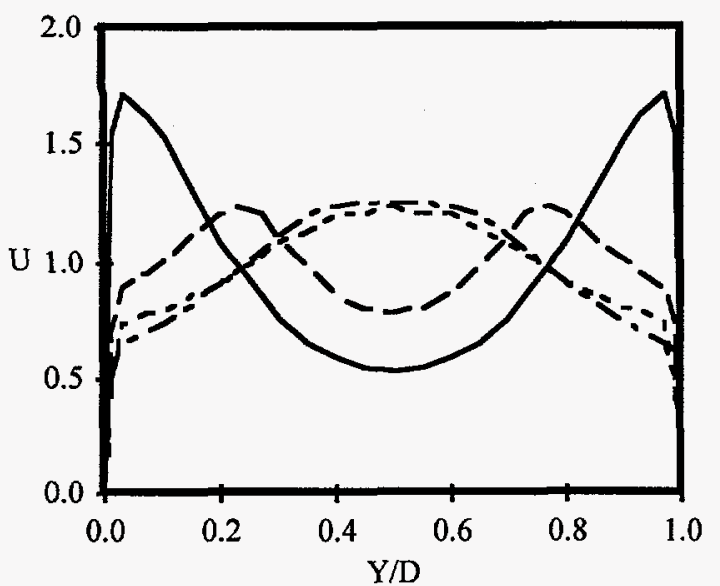

(a) Exit Axial Velocity

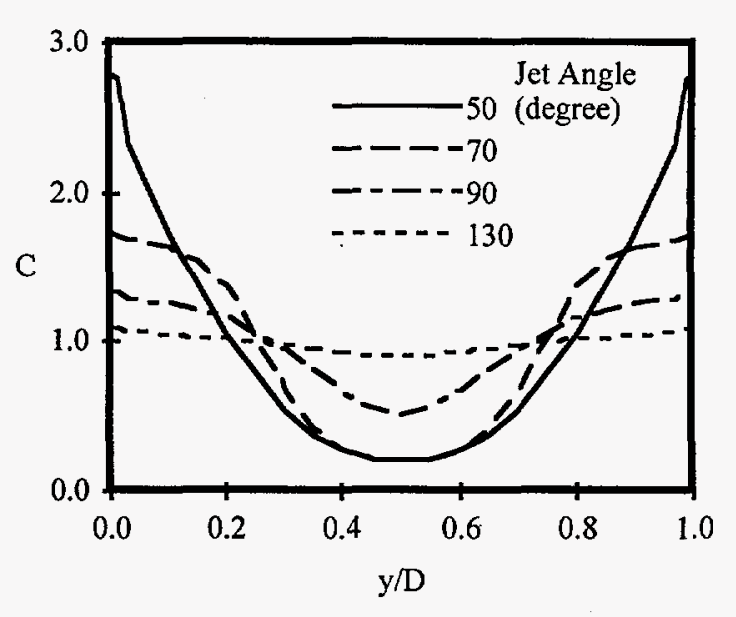

(b) Exit Jet Fluid Concentration

Figure 21 Effect of Injection Angle on Velocity and Concentration Profiles at the Combustor Exit

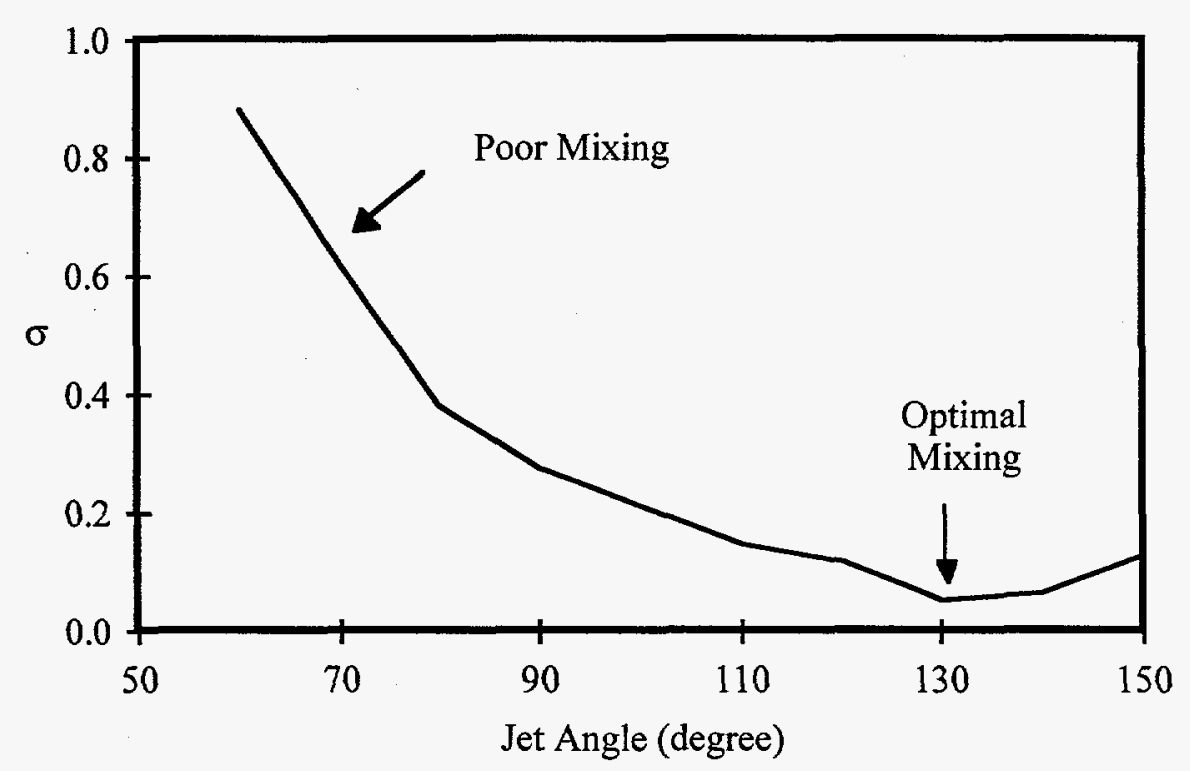

Figure 22 Effect of Injection Angle on Jet Fluid Mixedness at the Combustor Exit

\subsubsection{Effects of Jet Momentum}

Jet momentum plays a major role in the mixing development in the chamber. As jet momentum is increased, the jets penetrate deeper into the cross stream flow, and mixing between the jet fluid and cross stream fluid proceeds more rapidly. 
The effect of increasing jet momentum on the flow configuration that develops in the chamber is shown in Fig. 23. This figure shows the velocity vector field for four cases in which the jet velocity was varied from $30 \mathrm{~m} / \mathrm{s}$ to $300 \mathrm{~m} / \mathrm{s}$. Other conditions for these cases were the same as the baseline case (in particular, the injection angle was 130 degrees). For the case with $30 \mathrm{~m} / \mathrm{s}$ jets, Fig. 23a shows that the jets have little effect on the main flow. When the jet velocity is increased to $60 \mathrm{~m} / \mathrm{s}$, Fig. 23b, small vortices form downstream of the jets, but the effect of the jets on the overall flow field is still relatively small and occurs primarily in the region around the jets. When the jet velocity is increased to $150 \mathrm{~m} / \mathrm{s}$, the vortices increase significantly in size and the main flow is forced to accelerate through the chamber center to move past them (Fig. 23c). At $300 \mathrm{~m} / \mathrm{s}$ (Fig. 23d) the jets penetrate deeply into the chamber, creating a screen which produces a large acceleration of the main flow through the chamber center. In this case, the vortices formed behind the jets extend far into the downstream of the chamber (to near $x / D=$ 2.0).

The effects of these different flow patterns on the distribution of jet fluid in the chamber is shown in jet concentration contours plotted in Fig. 24, for the same four jet velocities, 30, 60, 150 , and $300 \mathrm{~m} / \mathrm{s}$. At low speed $(30 \mathrm{~m} / \mathrm{s})$, even with a counterflow injection angle of 130 degrees, the jets are turned rapidly toward the downstream of the main flow, and the jet fluid is primarily confined to a layer near the walls (Fig. 24a). When the jets do not penetrate effectively into the main stream, mixing downstream of the jets occurs primarily due to cross stream turbulent transport, and the turbulent transport mixing process is not fast enough to thoroughly mix the main and jet fluid streams within the short length of the chamber. The concentration distribution in this case of low-speed counterflow jets is similar to that of the case of high-speed co-flow jets (Fig. 19a). Both cases have relatively poor mixing with jet fluid confined in a region near the walls.

At a jet velocity of $60 \mathrm{~m} / \mathrm{s}$ (Fig. 24b) the layer near the walls of high jet fluid concentration is thicker than in the $30 \mathrm{~m} / \mathrm{s}$ case, indicating improved mixing, but there is still very little jet fluid reaching the chamber center. When the jet velocity is raised to $150 \mathrm{~m} / \mathrm{s}$, a significant amount of jet fluid reaches the chamber center before the chamber exit, but the concentration gradient of jet fluid across the exit, indicated by the number of contour lines, is still fairly large (mixing is still far from complete). The final plot of jet concentration contours for an injection velocity of $300 \mathrm{~m} / \mathrm{s}$ (Fig. 24d) shows jet fluid has reached the chamber center in the near downstream region beyond the jet ports. Further mixing over the remainder of the chamber length in the $300 \mathrm{~m} / \mathrm{s}$ case produces a fairly uniform mixture of jet and main flow fluid by the chamber exit. 


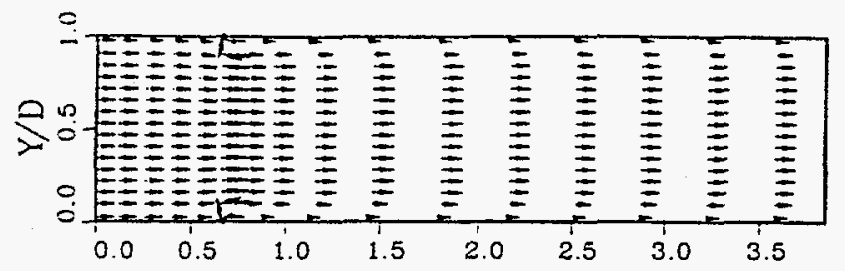

$\mathrm{XND}$

(a) Jet velocity $=30 \mathrm{~m} / \mathrm{s}$

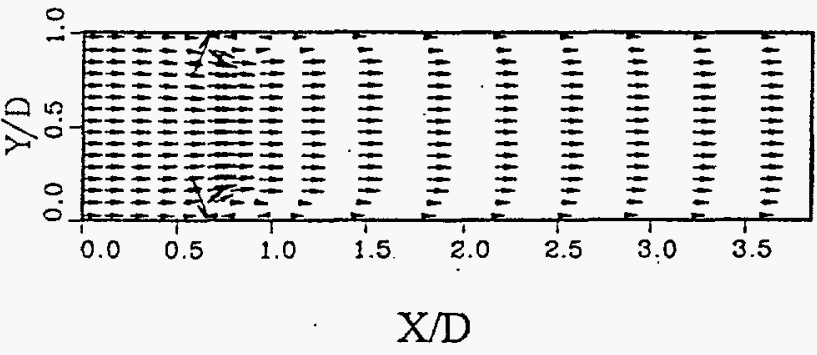

(b) Jet velocity $=60 \mathrm{~m} / \mathrm{s}$

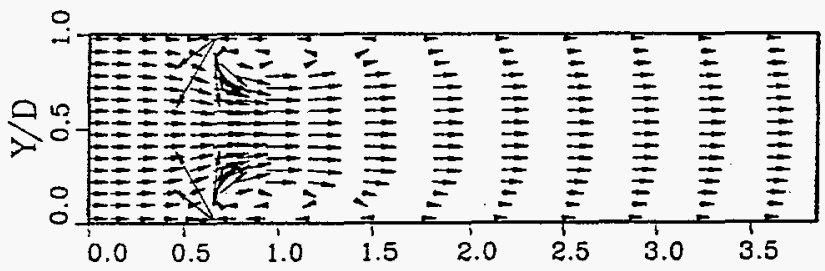

$\mathrm{X} / \mathrm{D}$

(c) Jet velocity $=150 \mathrm{~m} / \mathrm{s}$

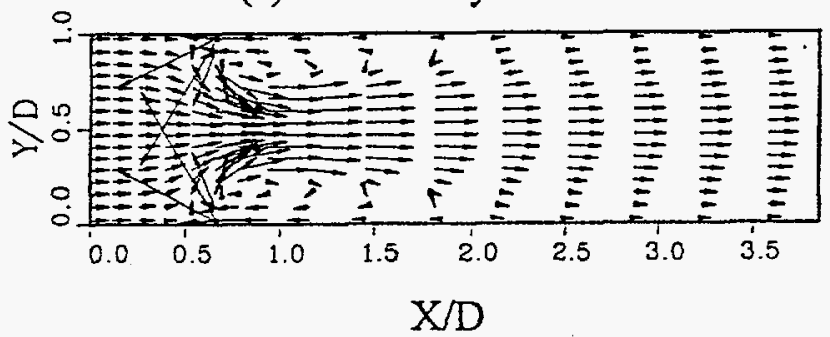

(d) Jet velocity $=300 \mathrm{~m} / \mathrm{s}$

Figure 23 Effect of Injection Velocity on Velocity.Field in the Combustor 


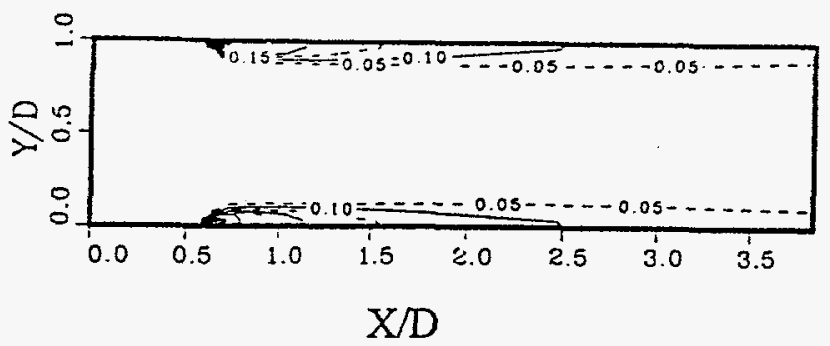

(a) Jet velocity $=30 \mathrm{~m} / \mathrm{s}$

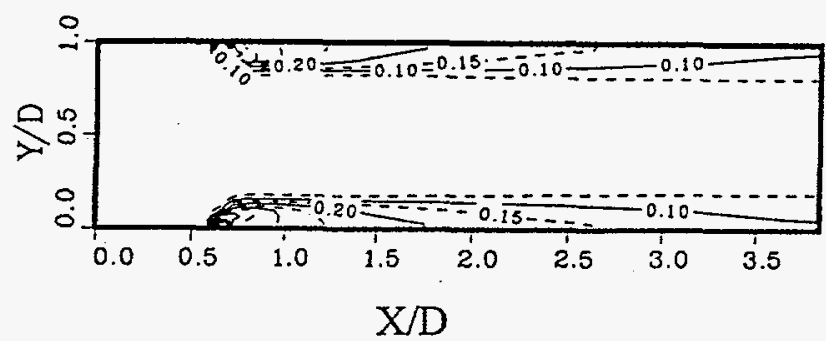

(b) Jet velocity $=60 \mathrm{~m} / \mathrm{s}$

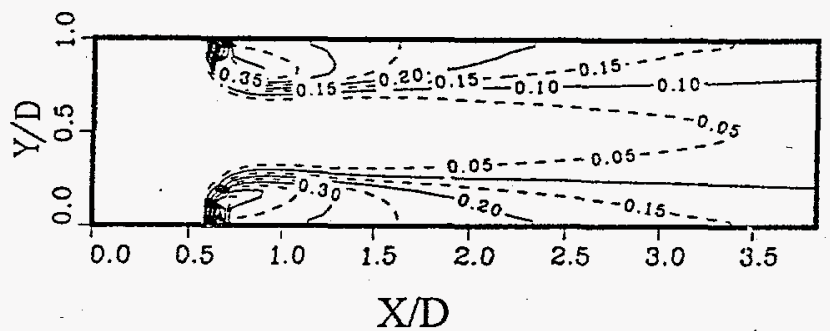

(c) Jet velocity $=150 \mathrm{~m} / \mathrm{s}$

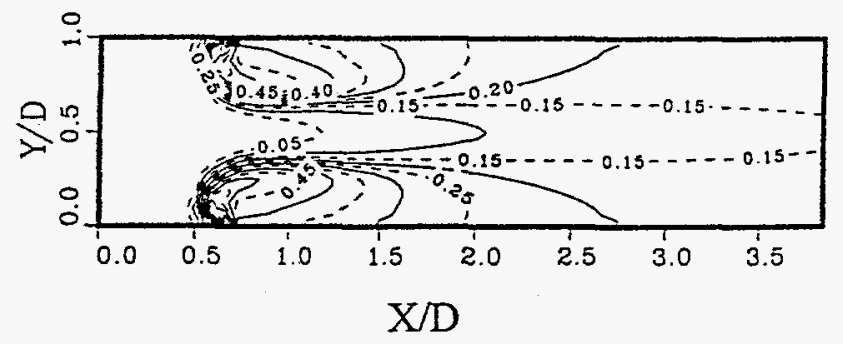

(d) Jet velocity $=300 \mathrm{~m} / \mathrm{s}$

Figure 24 Effect of Injection Velocity on Jet Penetration in the Combustor 
The overall mixing dependence on jet velocity can be seen most readily in plots of the normalized standard deviation of jet concentration over a chamber cross section (a measure of mixedness which becomes zero for completely mixed fluids). Figure 25 shows this mixedness measure plotted against the downstream distance in the chamber for four injection velocities (30, $60,150,300 \mathrm{~m} / \mathrm{s})$. These curves show the same kind of mixing development as those of Fig. $20 \mathrm{~b}$ : the region near the injector plane has a very high rate of mixing due to jet penetration into the main flow. Downstream of the injector plane, mixing occurs due to the much slower diffusive and turbulent transport processes. The importance to mixing of high speed, deeply penetrating jets is clearly apparent when comparing the curves of Fig. 25. Much of the mixing occurs in the vicinity of the jet ports and even in the region upstream of the ports in these cases of counterflow injection. In comparing the mixing curves of Fig. 25, the case with $300 \mathrm{~m} / \mathrm{s}$ is seen to achieve good mixedness by the exit primarily because mixing is very far advanced near the jet ports at $\mathrm{x} / \mathrm{D}=0.7$.

Results of a parameter sweep over cases of jet velocity ranging from $30 \mathrm{~m} / \mathrm{s}$ up to $300 \mathrm{~m} / \mathrm{s}$ are plotted in Fig. 26 for cases of counterflow injection at an injection angle of 130 degrees. This graph shows the normalized standard deviation of jet concentration near the exit plane at $\mathrm{x} / \mathrm{D}=3.8$, plotted using a logarithmic scale versus the jet injection velocity. The results indicate that the amount of mixing between jet fluid and main flow is strongly dependent on jet injection velocity, and that the higher the injection velocity, the better the mixing. The near linear curve on the semilog plot of Fig. 26 indicates that the data can be well correlated by an exponential function. A best fit of the data yields the relation:

$$
\sigma=1.54 \mathrm{e}^{-0.0108 \mathrm{v}_{\mathrm{j}}}
$$

This equation gives an approximate form of the relation between exit plane mixedness and jet injection velocity for base case conditions with an injection angle of 130 degrees.

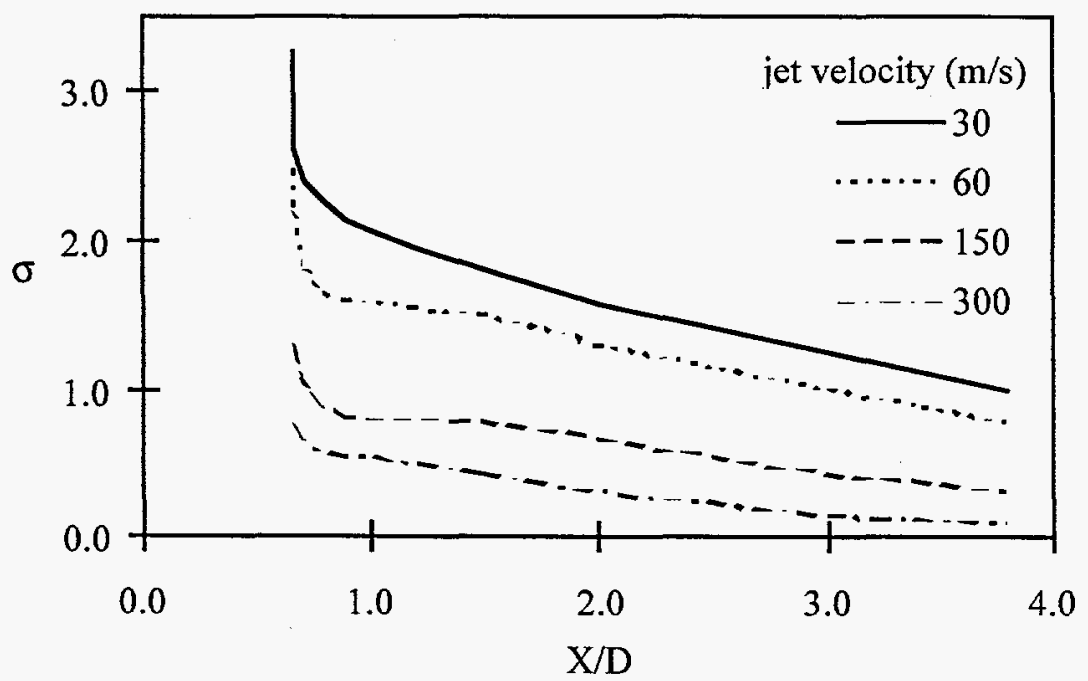

Figure 25 Effect of Injection Velocity on Jet Fluid Mixedness in the Combustor 


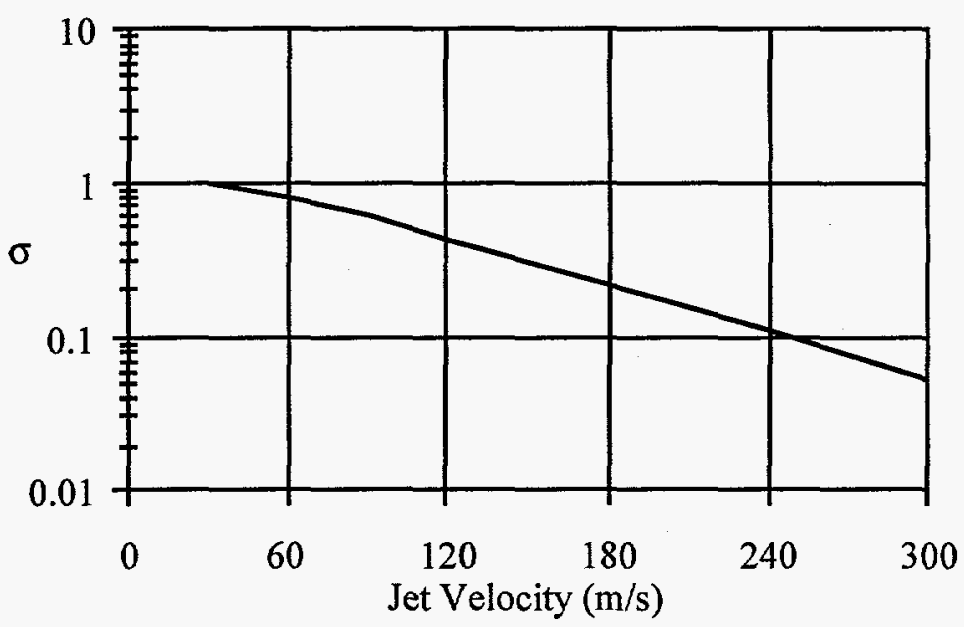

Figure 26 Effect of Injection Velocity on Jet Fluid Mixedness at the Combustor Exit

\subsubsection{Effects of Asymmetric Inlet Velocity Distribution}

In general, the inlet gas-flow velocity is asymmetric about the centerline because of the non-ideal deswirl process before entering the second-stage MHD combustor. Figure 27 shows the velocity vectors and jet concentration contour plots for a 130-degree case, where the inlet axial velocity changes linearly from $0.9 \mathrm{U}_{0}$ near $\mathrm{Y} / \mathrm{D}=0$ to $1.1 \mathrm{U}_{0}$ near $\mathrm{Y} / \mathrm{D}=1$, with other conditions remaining the same as in Table 4. Comparing Figs. 27 and 17 reveals that for the asymmetric inlet flow case, (1) the vortex near the bottom wall, Y/D $=0$, becomes larger, and (2) both maximum exit velocity and minimum exit jet-concentration shift to the upper half of the chamber. In the entrance region from the inlet to approximately $x / D=1$, the resultant momentum of both the inlet and the jet flows in the lower half of the chamber yields a higher pressure field compared to the upper-half flows, because of higher mass flow rate and velocity. The higher pressure field makes the main flow turn toward the upper-half chamber after the jet opening. Thus, a larger vortex is formed in the lower-half chamber and velocity peak shifts to the upper-half chamber.

The effect of this flow pattern on exit-plane velocity and jet-concentration profiles is to skew them slightly about the center; they are slightly higher for values of Y/D less than 0.5 , and corresponding for $\mathrm{Y} / \mathrm{D}$ values greater than 0.5 . For jet-mass mixing, the asymmetric case has a more uniform profile in the lower-half chamber and a less uniform profile in the upper-half chamber. These competing conditions yield an overall degree of jet-mass mixing with the main flow that is about the same in the symmetric and asymmetric cases. A comparison of the mixing development of these two cases along the chamber length shows that the diffusive and turbulent transport driven mixing of the asymmetric case is somewhat slower in the upstream and somewhat faster in the downstream in comparison to the symmetric case, yielding approximately the same amount of mixing over the chamber length. 


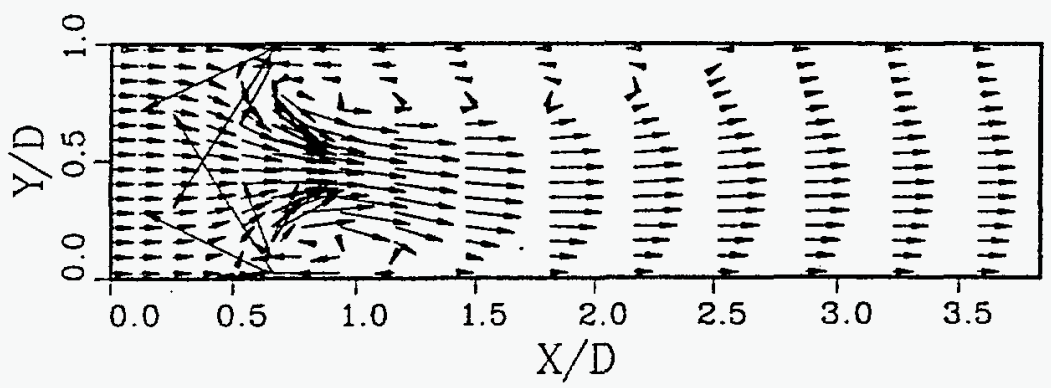

(a) Velocity vectors

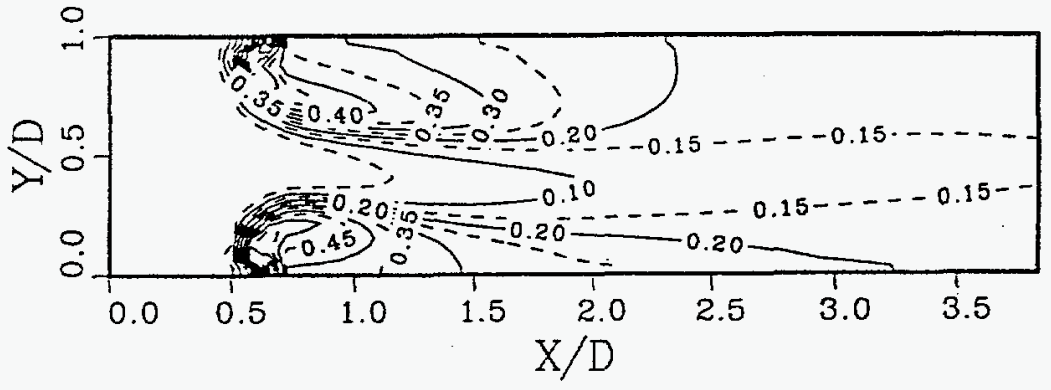

(b) Jet mass concentration

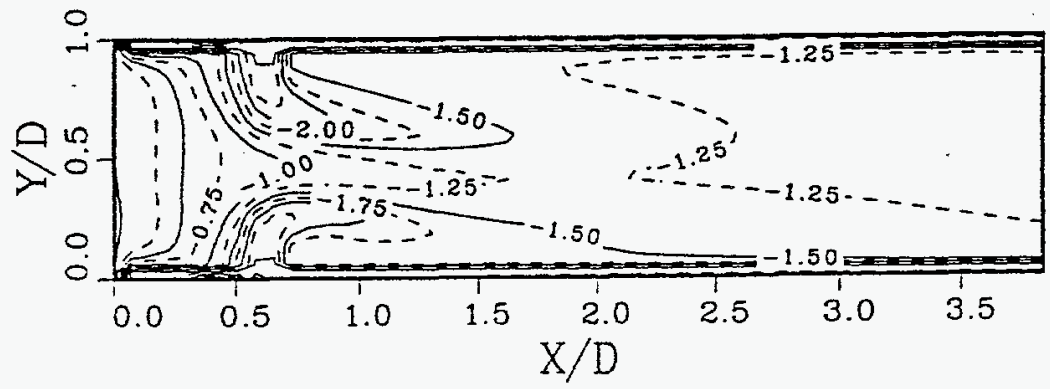

(c) Eddy size

Figure 27 Effect of Asymmetric Inlet Velocity on Nonreacting Flow Patterns in the Combustor 
A parametric sweep was performed, where the degree of velocity asymmetry at the chamber inlet ranged from symmetric to an inlet velocity profile varying $30 \%$ above and below the mean. While some variation of the mixedness measure was apparent, the variation of mixedness is small when compared to the variation caused by either the jet injection angle or the jet velocity parameters. Therefore, effects of inlet flow asymmetry within a range of $\pm 30 \%$ velocity variation appear to have only a secondary effect on the mixing of jet and main flow fluids over the length of the chamber.

Results of the parametric studies indicated (1) jet/main flow mixing strongly depends on jet angle in the jet angle range of 40-90 degrees, (2) counterflow jets have better jet/main flow mixing and mixing is relatively insensitive to jet angle (angle $>90$ degrees), (3) mixing increases as jet momentum increases, and (4) asymmetry of the inlet gas flow affects the mixing pattern but has a relatively small effect on the extent of jet/main flow mixing over the chamber length.

\subsection{Comparison with Experiment}

Experimental cold flow studies of the flow and mixing patterns in the second-stage of an MHD coal-fired combustor have been conducted by TRW $[15,16]$. A one-third scale transparent model of the 50-MWt combustor was used. In order to determine the degree to which the secondary oxidizer mixes with the combustion products from the first-stage, injector concentration measurements were taken throughout the flow cross-section at several axial stations downstream of the injector frame.

The greatest problem in matching the results of experiments was to simulate the correct jet penetration depth and mixing rate. If the simulated jet mass flow rate equals the measured jet mass flow rate and the simulated jet velocities equal the measured jet velocities, then the simulated jet penetration depth is much too shallow and mixing is much too slow. This shortfall may be attributed to the fact that distributing the round jets as a simulated slot increases the jet perimeter by a factor of 2.8 and decreases the jet width by a factor of 0.04 . These two factors allow cross-stream momentum from the main flow to penetrate the simulated jet slot much too quickly.

Table 5 shows the relation between jet mass flow rate and the ratio of three-dimensional round jet Reynolds number and simulated two-dimensional model slot jet Reynolds number. The mass flow rate of $0.01 \mathrm{~kg} / \mathrm{s}$ matches the TRW experimental conditions. In this case, the two-dimensional slot idealization of the round jets for purposes of two-dimensional computational simulation yields a large jet Reynolds number mismatch. To determine the effect that this Reynolds number mismatch has on the simulated results in comparison with experimental results, the Reynolds number ratio was reduced by tripling and then doubling the jet mass flow rate. Figure 28 shows exit jet mass concentration profiles for the case where the round jet and slot Reynolds numbers approximately match compared with TRW experimental data averaged over chamber depth to eliminate the dimension not modeled in the twodimensional computations. The figure shows that when jet Reynolds number similarity is 
preserved, ANL's two-dimensional simulation results come very close to TRW's experimental results. Except where Reynolds number related parameters were being investigated (such as injection velocity), Reynolds number similarity was preserved in the parametric studies of second combustor mixing presented previously in this chapter, and those which will be presented in following chapters.

Table 5 Relationship Between Jet Mass Flow Rate and Reynolds Number

\begin{tabular}{|c|c|c|c|}
\hline $\mathrm{m}_{\mathrm{i}}$ & 0.01 & 0.03 & 0.06 \\
\hline $\mathrm{Re}_{\mathrm{r} j} / \mathrm{Re}_{\mathrm{si}}$ & 5.0 & 1.7 & 0.81 \\
\hline
\end{tabular}

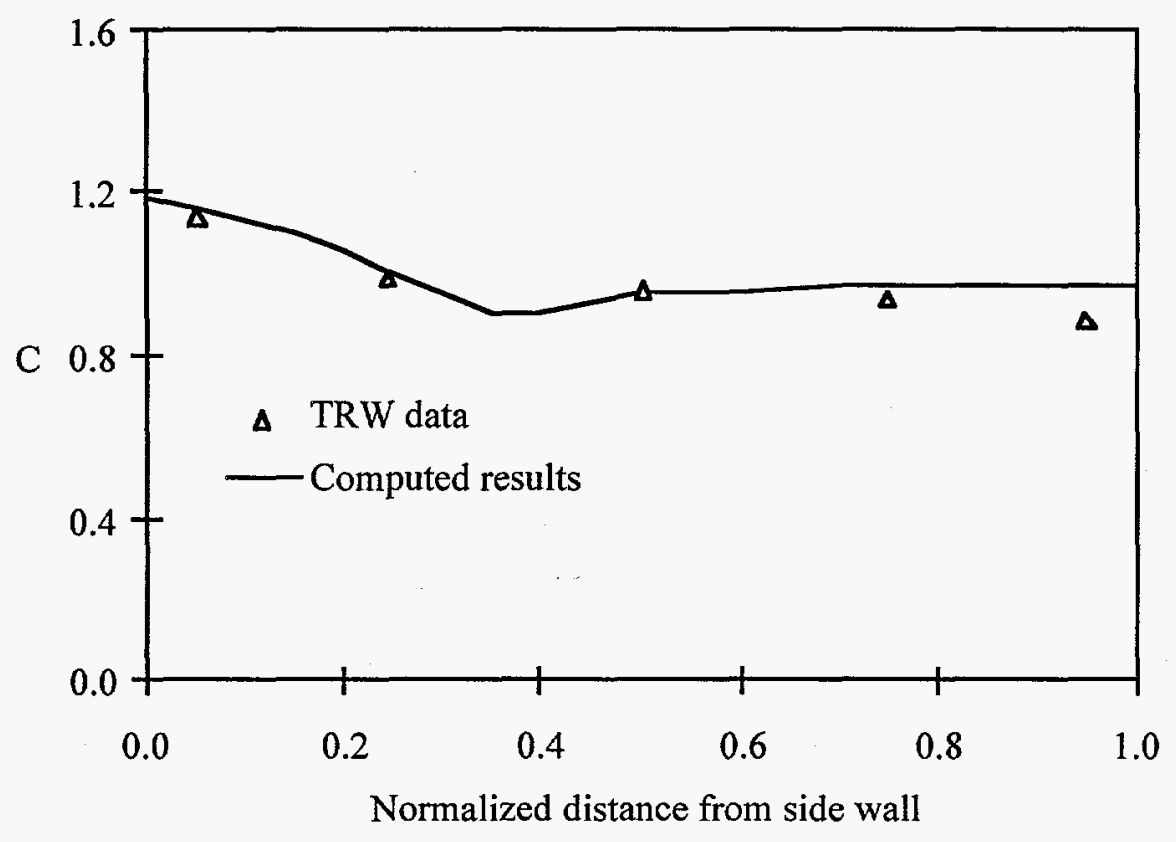

Figure 28 Effect of Injection Flow Rate on Jet Fluid Mixedness at the Combustor Exit 


\section{REACTING FLOW IN THE SECOND-STAGE COMBUSTOR}

Streams of oxidizer (pure oxygen) and coal-gas mix and burn in the MHD second-stage combustor. The completeness of combustion has a great impact on the performance of the MHD power generation system. The nonreacting flow ICOMFLO computer code was expanded by incorporating a new lumped integral combustion submodel. The incorporation of the new reaction submodel makes numerical calculations of a reacting flow more efficient and more stable while still preserving the major physical effects of the complex combustion processes. The modification of the reacting flow code (now called ICOMFLO) for the simulation of the MHD second-stage combustion is described in more detail in the literature [21,22]. A parametric study was conducted to investigate the effects of operating conditions, e.g., injection angle, on the completeness of combustion. Results of the parametric study indicate (1) fluid mixing is mainly responsible for combustion performance and (2) counter-flow injection, with an injection angle in the range of 120 to 140 degrees, yields the best mixing and combustion performance. These were confirmed by test results collected at the CDIF.

\subsection{Reacting Flow System}

Figure 29 shows the 2-D computational domain whose boundaries include an inlet (left dashed line), an exit (right dashed line), and two injector slots (arrows) on the top and the bottom side walls. An injection angle $\theta$ is defined as the angle between the bottom wall in the main flow direction and the bottom jet vector. The inlet stream consists of coal gas and inert gas. The jet streams are oxidizer. Coal gas and oxidizer burn in the combustor and generate product.

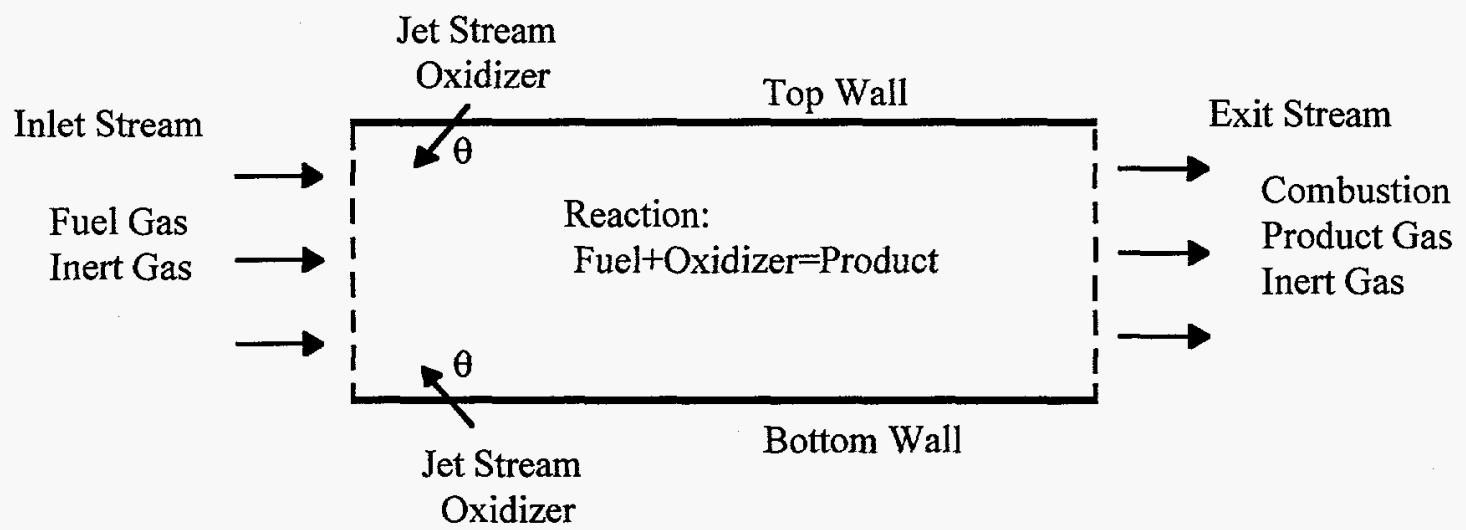

Figure 29 Computational Domain of 2-D Combustion Flow in the MHD Second-stage Combustor

\subsection{Lumped Integral Combustion Submodel}

The ICOMFLO computer code described in Chapter 3 was developed primarily for nonreacting flows, although it contained a poorly functioning Arrhenius reaction submodel. A new lumped combustion submodel was added to the computer code so it could be used to simulate the 
reacting flow in the second-stage combustor. Calculations of a combustion computer code have long been troubled by numerical convergence and instability problems. In a combustion flow, the reaction time scale is generally much smaller than a flow time scale. The difference of the reaction and flow time scales is believed to cause the numerical problems. A conventional combustion flow computer code uses an Arrhenius equation to represent the reaction rate. The Arrhenius reaction rate is expressed in a differential form. When the reaction time scale is much smaller than the flow time scale, the Arrhenius reaction rate becomes extremely large in a combustion flow calculation; the Arrhenius reaction rate cannot take into account the changes (reaction) that occur as the flow crosses a computational cell, since the cell size and machine precision determine the degree of property and process resolution in the computational domain. Using hundreds of thousands of very small cells cannot solve this scale difference problem because machine precision limits the accuracy to which gradients can be calculated on a very fine grid (hydrodynamic gradients may be lost in the noise of round off error on a doubleprecision, 14-digit, machine with extremely fine grid). Using a grid that is optimal for hydrodynamic calculations, however, causes the Arrhenius rate formula to greatly overpredict the mean reaction rate over the flow residence time for a computational cell with a high reactant influx rate, and this overestimation leads to numerical instability.

A new lumped integral combustion submodel was developed to replace the old Arrheniustype differential reaction rate submodel. The integral submodel assumes that the overall reaction progress and its physical effects can be expressed by empirical correlations or tabulated data relating the extent of reaction (or the fraction of fuel consumed) and the accumulated heat of combustion to a flow time scale. The correlations or data tables are derived from the results of detailed kinetics calculations, which provide data to tabulate or correlate an extent of reaction as a function of time. The detailed kinetics calculations can be carried out by a separate kinetics computer code, for example, NASA's General Chemical Kinetics Program [23]. An accumulated heat of combustion, which is the heat release rate integrated from the initial time to a specific lag time of the reaction, can also be obtained from the kinetics calculation.

The combustion processes of the MHD second-stage combustor may involve 14 species in 30 reactions [24]. Direct application of these reactions to a CFD code can cause severe numerical instability problems. A simple four-lump reaction (Eq. 4.1) is used to represent the combustion. Fuel, oxidizer, product, and inert are the four species lumps. Fuel and oxidizer react and generate product. The inert species is not involved in the reaction.

$$
\text { fuel }+ \text { oxidizer }+ \text { inert } \longrightarrow \text { product }+ \text { inert }
$$

Table 6 lists the inlet flow conditions of the MHD second-stage combustor. The inlet gas temperature is $1750 \mathrm{~K}$ and the pressure is $5.7 \mathrm{~atm}$. The inlet gas is a mixture of $\mathrm{CO}(27.2 \%), \mathrm{H}_{2}$ (8.1\%), $\mathrm{O}_{2}(17.7 \%), \mathrm{CO}_{2}(7.5 \%), \mathrm{H}_{2} \mathrm{O}(10 \%)$, and $\mathrm{N}_{2}(29.5 \%)$. The chemical formulae of the lumped species are derived from the inlet flow compositions. Fuel is defined as $\mathrm{CH}_{0.6} \mathrm{O}$, oxidizer is $\mathrm{O}_{1.3}$, product is $\mathrm{CH}_{0.6} \mathrm{O}_{2.3}$, and inert species is $\mathrm{N}_{2}$. The chemical formulae, molecular weights, and specific heats of the four-lumped species are listed in Table 7. 
Table 6 Inlet Flow Properties of the Second-stage Combustor

\begin{tabular}{|l|c|}
\hline \multicolumn{1}{|c|}{ Flow Properties } & Property Value \\
\hline Temperature $(\mathrm{K})$ & 1750 \\
\hline Pressure $(\mathrm{Pa})$ & $5.83 \times 10^{5}$ \\
\hline Gas Composition (mole fraction) & \\
\hline $\mathrm{CO}$ & 0.272 \\
\hline $\mathrm{H}_{2}$ & 0.081 \\
\hline $\mathrm{O}_{2}$ & 0.177 \\
\hline $\mathrm{CO}_{2}$ & 0.075 \\
\hline $\mathrm{H}_{2} \mathrm{O}$ & 0.100 \\
\hline $\mathrm{N}_{2}$ & 0.295 \\
\hline
\end{tabular}

Table 7 Molecular Weights and Specific Heats of the Lumped Species

\begin{tabular}{|l|c|c|}
\hline Lumped Species & $\mathrm{w}(\mathrm{kg} / \mathrm{kmol})$ & $\mathrm{Cp}(\mathrm{J} / \mathrm{kmol}-\mathrm{K})$ \\
\hline Fuel $\left(\mathrm{CH}_{0.6} \mathrm{O}\right)$ & 28.60 & 37,630 \\
\hline Oxidizer $\left(\mathrm{O}_{1.3}\right)$ & 20.76 & 17,560 \\
\hline Product $\left(\mathrm{CH}_{0.6} \mathrm{O}_{2.3}\right)$ & 49.36 & 55,200 \\
\hline Inert $\left(\mathrm{N}_{2}\right)$ & 28.00 & 29,110 \\
\hline
\end{tabular}

The extent of reaction $\zeta$ is defined as the mass ratio of the fuel consumed to the original amount of fuel. This parameter is particularly important in representing a combustion process because it decreases monotonically with the reaction time and is bounded below by zero. The extent of reaction for the lumped species reaction (Eq. 4.1) is defined as

$$
\zeta=-\frac{1}{Y_{f, o}} \int_{o}^{t} \frac{d Y_{f}}{d t} d t
$$

where $t$ is the reaction time and $Y_{\mathrm{f}, 0}$ is the initial fuel concentration.

Extent of reaction data for the detailed multi-step reaction mechanism of the MHD secondstage combustion is computed using NASA's kinetics computer code. The computed timedependent temperature (solid line) and extent of reaction (dashed line) are plotted in Fig. 30. The temperature value in the figure is normalized by an adiabatic flame temperature, $\mathrm{T}_{\mathrm{a}}=3000 \mathrm{~K}$. The results show that temperature increases from $1750 \mathrm{~K}$ to $2985 \mathrm{~K}$ in about $4 \mu \mathrm{s}$, and in the same time, the extent of reaction increases from 0 to 0.57 . At the end, $43 \%$ of the fuel remains, due to high-temperature dissociation. 


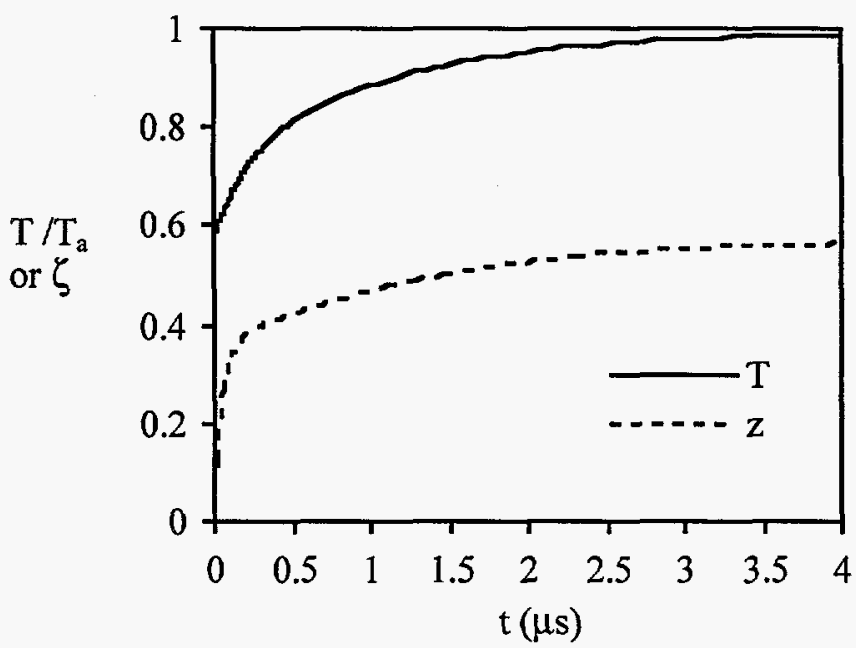

Figure 30 Progress of Reaction Derived from Kinetics Calculation

The computed extent of reaction can also be correlated as a function of reaction time.

$$
\begin{array}{lc}
\zeta=0.1495 \ln (80 t+1) & t \leq 0.1 \mu s \\
\zeta=1-\exp \left(-0.705 t^{0.25}\right) & 0.1<t \leq 0.25 \mu s \\
\zeta=0.57-0.1774 \exp \left[-0.78(t-0.25)^{1.04}\right] & t>0.25 \mu s
\end{array}
$$

in which reaction time is expressed in $\mu$ s. Similarly, accumulated heat of combustion can be computed from the results and correlated as a function of the extent of reaction. The correlation for accumulated heat of combustion is

$$
\begin{array}{ll}
H_{f}=0.001 \exp (9.52 \zeta) & \zeta \leq 0.393 \\
H_{f}=56.9-\exp (0.57-\zeta) & \zeta>0.393
\end{array}
$$

The reaction time scale, about $4 \mu \mathrm{s}$, is much smaller than the flow time scale on the hydrodynamic computational grid, which is on the order of milliseconds in this case. The big difference between these two time scales has caused severe numerical problems in the previously employed differential reaction submodels. To prevent this problem from occurring, the new reaction flow submodel expresses heat release and fuel consumption in a flow time scale by integrating over the reaction time scale. In the new computer code, the time-resolved extent of reaction is formulated as a sink term for the fuel species equation. As for the heat of combustion, the source term, including the empirical accumulated heat of combustion, is formulated for the enthalpy equation.

\subsection{Effects of Operating Conditions on Combustion}

The non reacting flow studies discussed in Chapter 3 showed that the flow configuration in the interior of the chamber depended strongly on jet injection angle. Two different flow regimes were identified, one for co-flow injection, where jet mass and momentum are turned sharply 
downstream, forming thick layers near the walls and yielding poor mixing with the main flow at chamber center, and one for counter-flow injection, where jets penetrate deeply, vortices are formed behind the jets, and much better mixing occurs between jet and main-flow fluids. Simulations were performed to determine flow configurations under reacting flow conditions and in particular to determine if the injection angle interval predicted as best for mixing under nonreacting flow conditions would correspond to the injection angle intervals that would be predicted as best for combustion. In evaluating the combustion, two desirable characteristics are considered: (1) the uniformity of the temperature profile at the exit plane, and (2) the completeness of combustion at the exit plane.

The common flow conditions for the two-dimensional reacting flow computations are summarized in Table 8. Some of the reacting flow results are compared to previous nonreacting flow jet mixing computation results.

Table 8 General Reacting Flow Conditions in the Second-Stage Combustor

\begin{tabular}{|l|c|}
\hline Inlet Temperature $(\mathrm{K})$ & 1974 \\
\hline Jet Temperature $(\mathrm{K})$ & 300 \\
\hline Jet Location $\left(\mathrm{L}_{\mathrm{j}} / \mathrm{D}\right)$ & 0.66 \\
\hline Jet Velocity/ $\mathrm{U}_{0}$ & 13.7 \\
\hline Pressure (atm) & 5.7 \\
\hline Overall Stoichiometric Ratio & 1.0 \\
\hline Inlet Fuel Mass Fraction & 0.38 \\
\hline Jet Oxidizer Mass Fraction & 1.0 \\
\hline Combustor Aspect Ratio (L/D) & 3.84 \\
\hline
\end{tabular}

\subsubsection{Effects of Jet Angle on Temperature Field}

The main characteristics of the reacting flow field are shown for 130-degree oxidizer jet injection in Fig. 31. The first three frames a, b, and c are contour plots of temperature, fuel mass fraction, and oxidizer mass fraction, respectively. Temperature is normalized by the inlet temperature of the main flow. The equilibrium temperature of the reaction under adiabatic conditions is about 1.5 times the main flow inlet temperature. The jet injection ports are located at $\mathrm{X} / \mathrm{D}=0.66$. With 130 -degree counter-flow injection, intense mixing occurs upstream of the injectors, and the flame is established in this intense mixing zone. Because the jets penetrate deeply into the main flow, large vortices form behind the jets (Fig. 31d), and the flame follows the vortex boundary from the upstream, where fuel and oxidizer first meet, around toward chamber center as the oxidizer jets are turned downstream. The flame then continues to develop as a diffusion flame in the mixing layer between fuel and oxidizer in the downstream. In Fig. 31, frames (b) and (c) show that much of the fuel and oxidizer are consumed by the exit plane, and frame (a) shows that the fluid temperature at the exit plane is relatively uniform and approaching the reaction equilibrium temperature. 
The characteristics of combustion with a 50-degree oxidizer jet injection angle contrast sharply with those of a 130-degree oxidizer jet injection angle, described above. Figure 32 shows plots corresponding to those of Fig. 31 for a case of combustion with a 50-degree oxidizer jet injection angle. As was the case in the nonreacting flow studies [14-17], for 50-degree injection the oxidizer jets do not penetrate significantly into the main flow, but rather are rapidly turned into the downstream forming a thick high gradient region near the walls. This flow configuration creates a nearly pure diffusion flame with a relatively low rate of combustion compared to the 130-degree injection case. As shown in Fig. 32, for 50-degree injection, oxidizer remains in a thick layer near the walls, and therefore the diffusion flame is also relatively nearer the walls, fuel is not depleted significantly from chamber center along its entire length, and heat released by combustion in the diffusion flame does not diffuse significantly to the chamber center either.

The dependence of exit-plane thermal mixedness on jet injection angle for reacting flow is compared to the dependence of jet mass mixedness for nonreacting flow in Fig. 33, which shows the normalized standard deviation of temperature (a), or jet mass concentration (b), at the exit plane plotted as a function of jet angle. The trends are similar. A large increase in exit plane mixedness (decrease in standard deviation) occurs when going from co-flow injection (angle $<90$ degrees) to counter-flow injection (angle $>90$ degrees). The angle range for most complete thermal mixedness in reacting flow (approximately 120 to 140 degrees) also matches the angle range for most complete jet mass mixedness in nonreacting flow.

In the reacting flow, a particularly large increase in exit thermal mixedness (decrease in standard deviation) occurs when the injection angle is increased from 70 to 80 degrees (Fig. 33a). Figure 34 shows that the injection angle range from 70 to 80 degrees includes the transition from the flow regime characteristic of co-flow injection, with small vortices behind the jets, a double humped velocity profile in the downstream, and relatively poor mixing into the central region, to the flow regime characteristic of counter-flow injection, with relatively large vortices behind the jets, a high velocity accelerating flow in the central region, and much better mixing into the central region. 

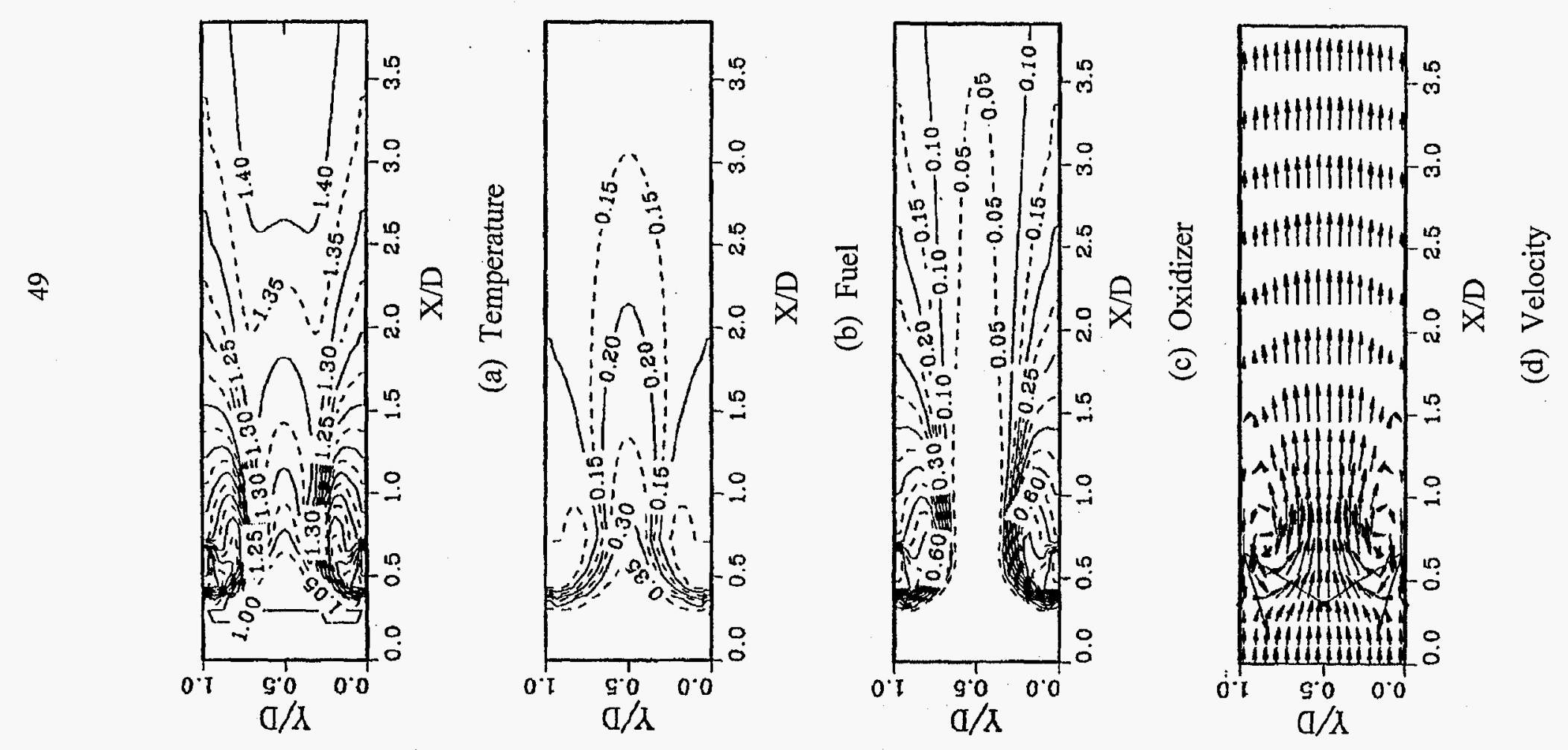

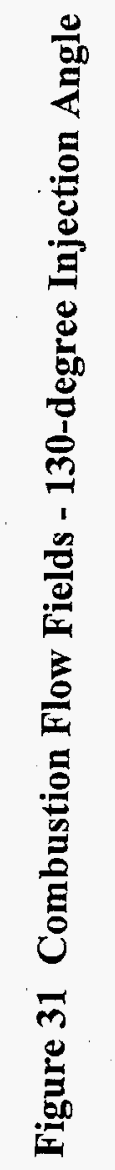




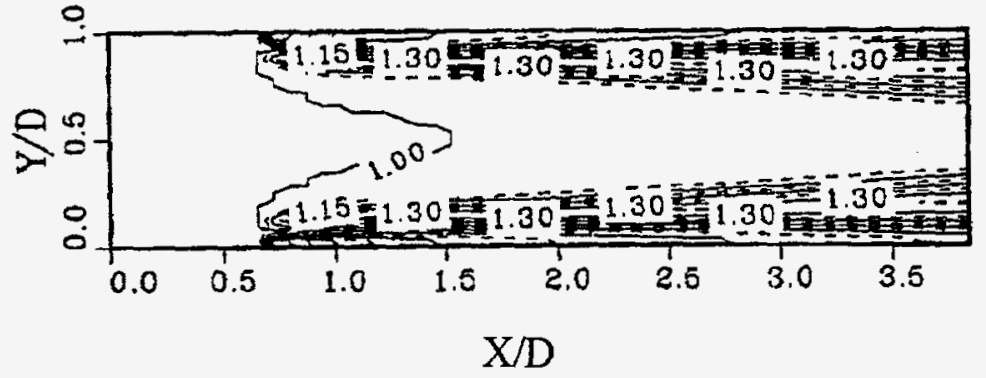

(a) Temperature

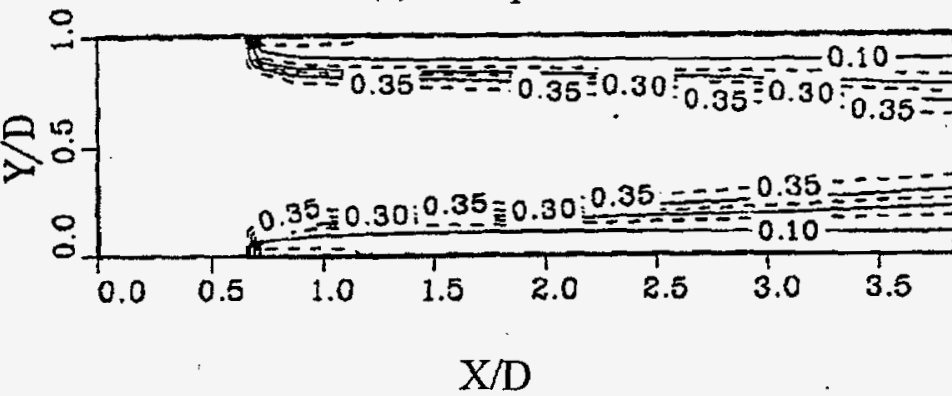

(b) Fuel

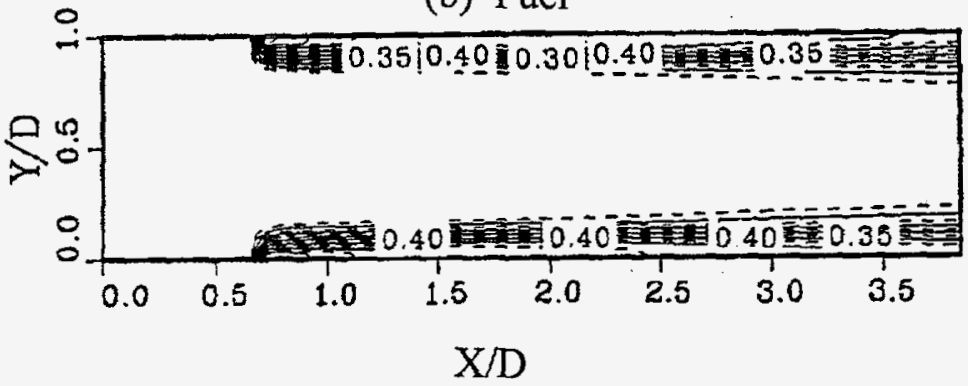

(c) Oxidizer

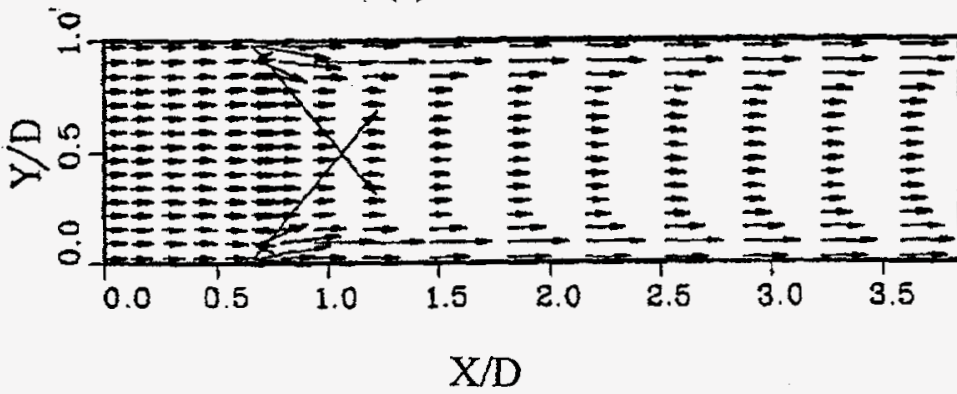

(d) Velocity

Figure 32 Combustion Flow Fields - 50-degree Injection Angle 


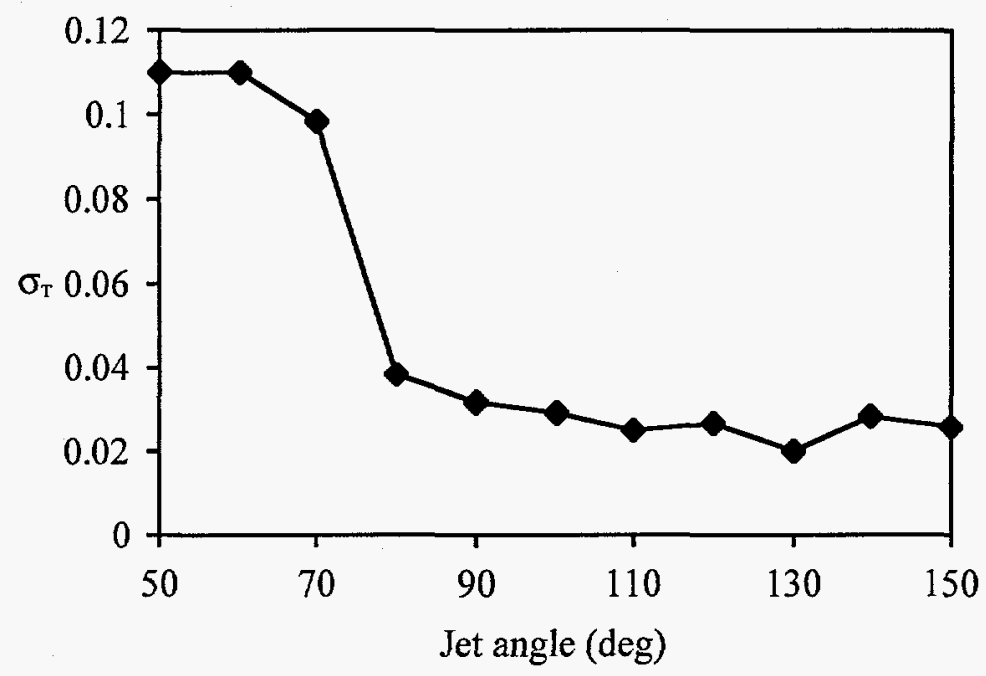

(a) Enthalpy Mixedness

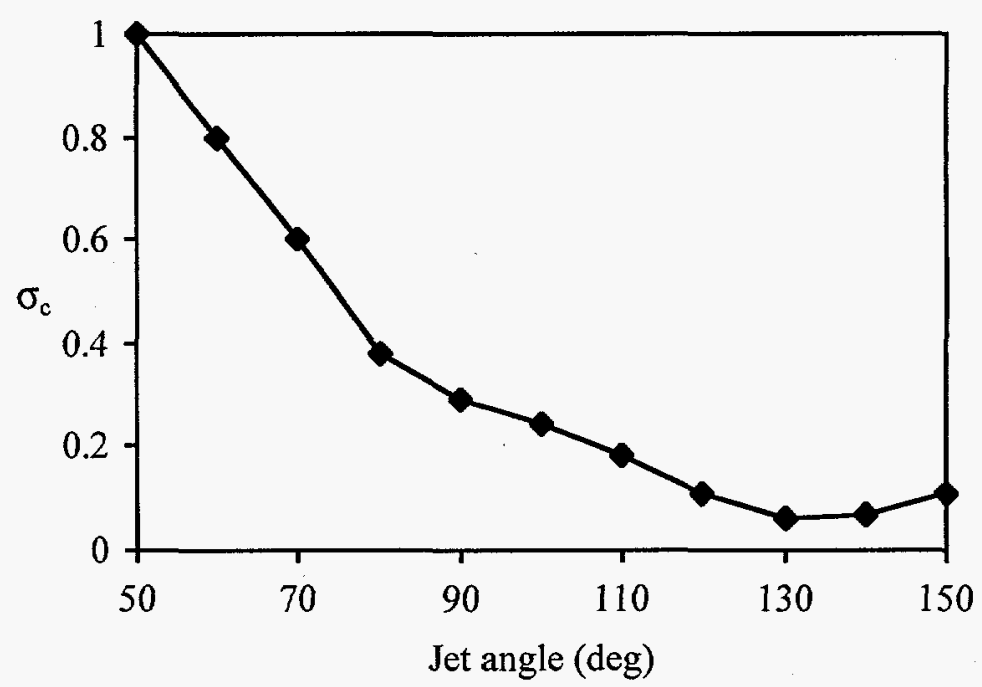

(b) Fluid Mixedness

Figure 33 Effect of Injection Angle on Enthalpy and Fluid Mixedness in the Combustor 

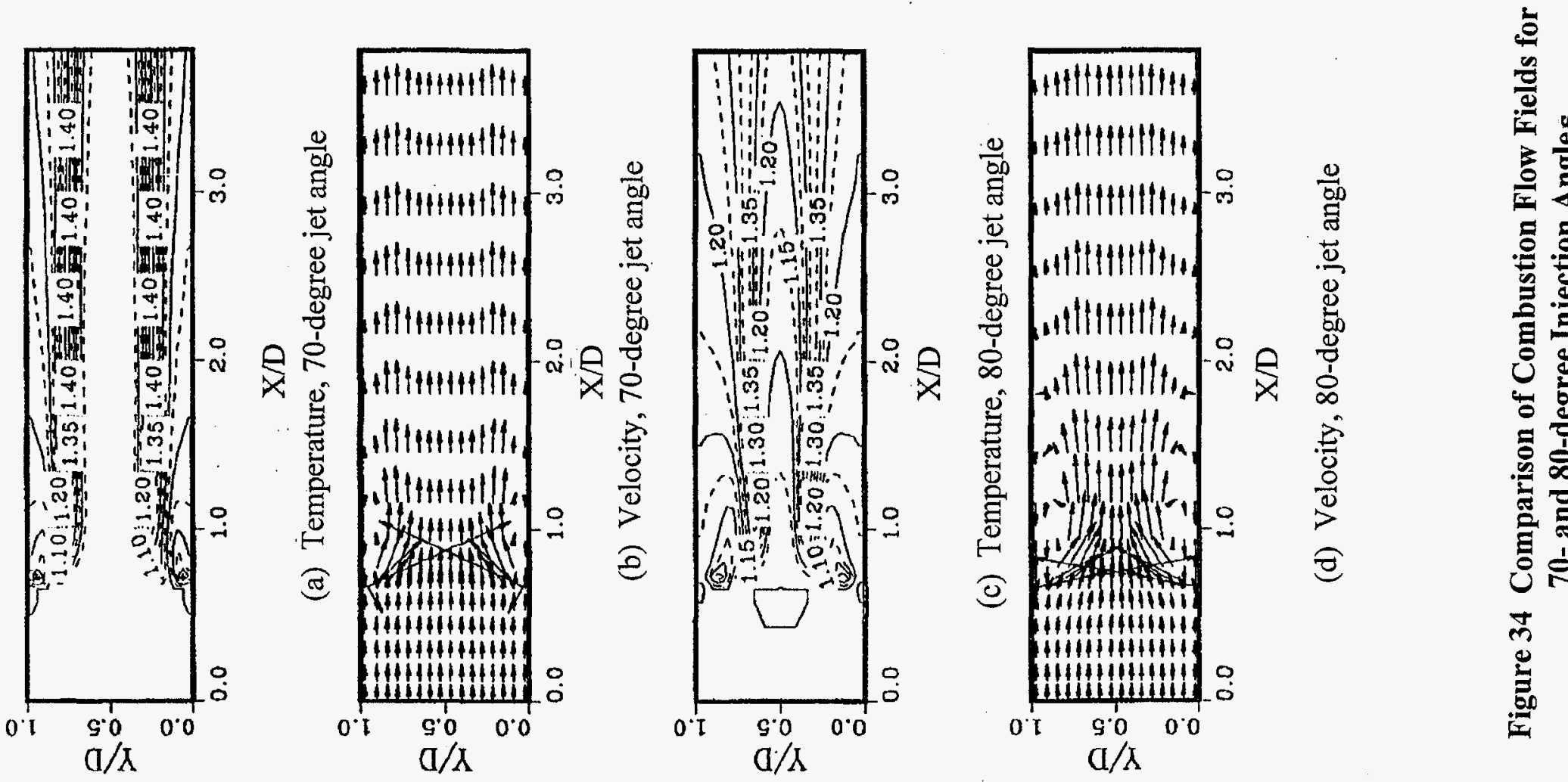
The development of the mean temperature and normalized standard deviation of temperature along the main flow or $\mathrm{x}$-direction for four different injection angles is shown in Fig. 35. These angles include the co-flow (50-degree) and counter-flow (130-degree) test angles of TRW, and the 70-and 80-degree injection angles, which approximately bound the transition of flow regimes from that characteristic of co-flow injection to that characteristic of counter-flow injection. The mean cross section temperature increases along the chamber length as combustion dumps heat into the fluid. In the angle range shown in Fig. 35a, higher injection angles yield better mixing overall and more complete combustion, and therefore higher mean temperatures.

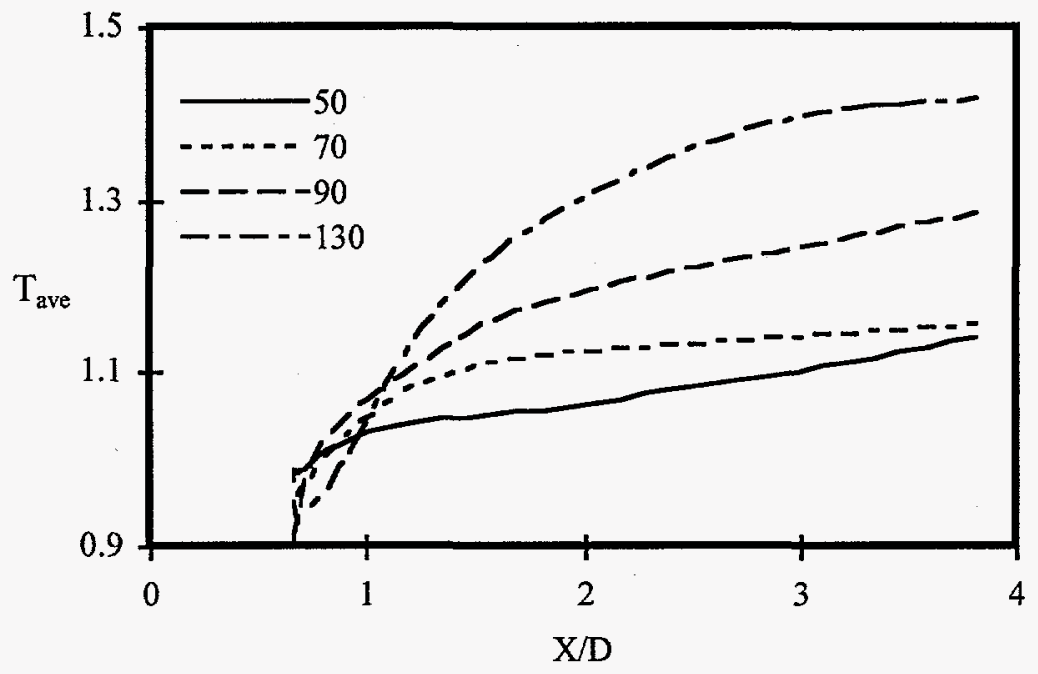

(a) Normalized Cross-section Mean Temperature

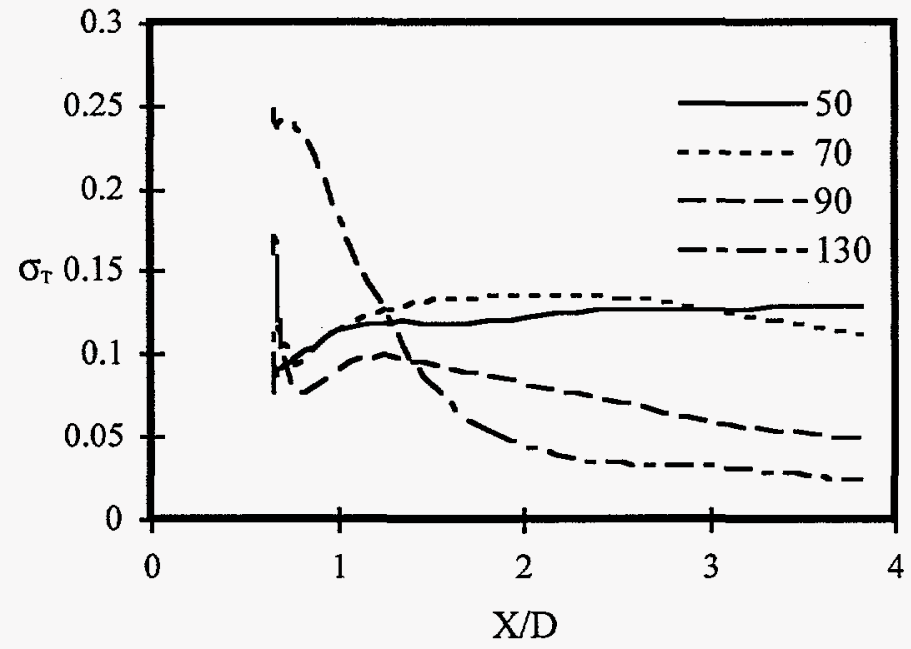

(b) Enthalpy Mixedness

Figure 35 Effect of Injection Angle on Axial Enthalpy Mixedness in the Combustor 
Because combustion is a thermal source in the flow field, thermal mixedness may decrease (standard deviation may increase) in the main flow direction as heat is added locally at the flame locations in a cross section. Combustion and mixing, then, are competing effects contributing to the normalized temperature deviation, $\sigma_{\mathrm{T}}$. For some cases shown in Fig. 35b, the standard deviation increases over some distance downstream of the injectors as combustion dumps heat into the fluid at the local flame zone faster than it is mixed; then the deviation decreases in the downstream as the combustion rate slows due to depletion of reactants and as mixing continues to smooth the temperature profile. The development of temperature standard deviation for the case of 130-degree injection in Fig. 35b is considerably different from the other cases shown. In the 130-degree injection case, the jets penetrate deeply into the chamber. This deep jet penetration pushes the diffusion flame much closer to the center than in the other cases, with a consequent greater localization of the combustion heat source near the chamber center. The recirculation zones behind the jets in this case become relatively cool pockets. As a result, the cross section temperature standard deviation for the 130-degree injection case is significantly higher than for the other cases in the region just downstream of the injectors. The rapid mixing induced by 130 -degree injection, however, is sufficient to bring the temperature standard deviation below those of the other cases by the time the flow reaches the exit plane.

The temperature standard deviations for the reacting flows of Fig. 35b can be compared with the jet fluid mass concentration standard deviations for nonreacting flows of Fig. 20. Because much of the mixing of jet fluid occurs upstream of the injectors for the 130-degree counter-flow, nonreacting flow case, the jet concentration deviation is already very low at the injector plane at $\mathrm{X} / \mathrm{D}=0.66$.

\subsubsection{Effects of Jet Angle on Combustion Efficiency}

The second desirable characteristic considered in evaluating the reacting flow regimes for different injection angles was the completeness of combustion. Figure 36 shows the main flow or X-direction development of combustion efficiency defined as the mass fraction of fuel consumed, $\eta$, from the combustor entry and a given cross section, $X / D$, for four different injection angles. For all three injection angles less than 90 degrees, the flame front develops from the injection ports at $\mathrm{X} / \mathrm{D}=0.66$ into the downstream, which contributes to a combustion completeness that lags the 130-degree injection case. In the 130-degree injection case, the counter-flow injection allows the formation of the flame front upstream of the injectors. More rapid mixing in the 130-degree injection case also appears to produce a sustained higher combustion rate in the diffusion flame downstream of the injectors, as indicated by the steeper slope of the combustion efficiency curve for the 130-degree case in the downstream region before the asymptotic approach to equilibrium conditions at about $\mathrm{X} / \mathrm{D}=3.0$. The cases for injection angles less than 90 degrees fall well short of approaching equilibrium combustion conditions at the chamber exit. 


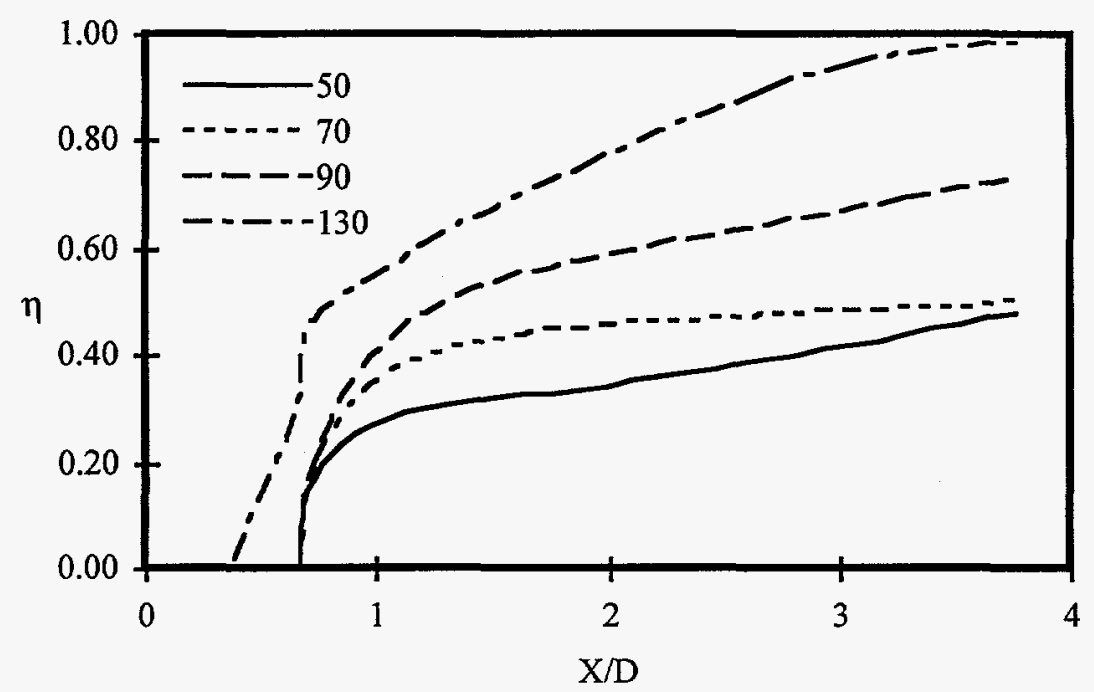

Figure 36 Effect of Injection Angle on Combustion Efficiency in the Combustor

Combustion efficiency at the chamber exit plane as a function of oxidizer injection angle is plotted in Fig. 37. In general counter-flow jet injection (angle $>90$ degrees) produces much higher exit plane combustion completeness than co-flow injection (angle $<90$ degrees). Both upstream penetration and penetration into chamber center serve to produce more complete mixing and combustion by the time the flow reaches the exit plane. Therefore, an optimum counter-flow injection angle range appears to exist. Counter-flow injection angles close to 90 degrees do not yield enough upstream penetration to be optimum, and angles beyond 150 degrees do not yield sufficient penetration into the chamber center to be optimum. The optimum injection angle range for combustion completeness appears to be in the interval from 120 to 140 degrees. As discussed previously, this range is also the injection angle range that produces the most uniform exit-temperature profile.

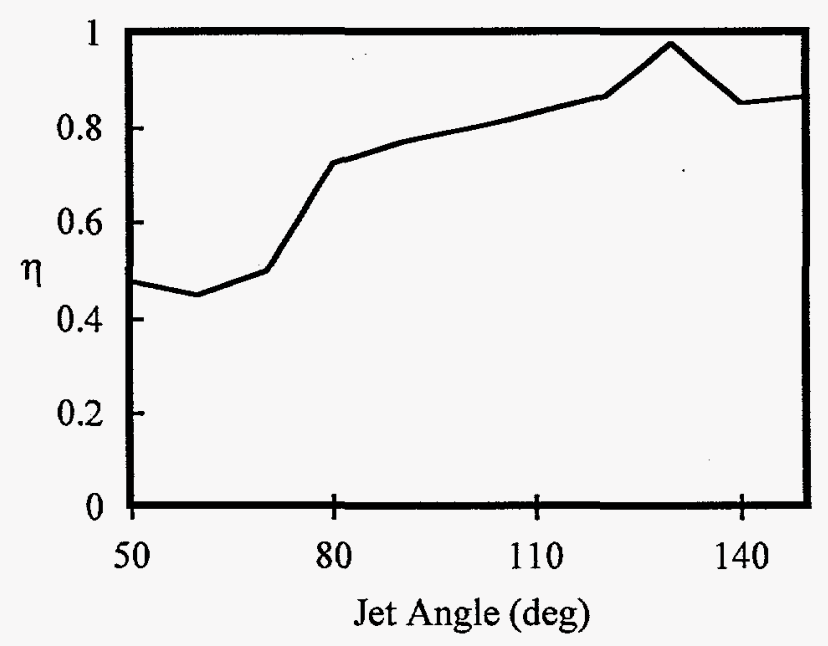

Figure 37 Effect of Injection Angle on Combustion Efficiency at the Combustor Exit 


\subsection{Numerical Convergence and Stability}

In a typical computation, the mass residual of each cell in the computational domain is checked after each iteration solving the governing equation set. The mass residual is required to be smaller than a preset convergence criteria before stopping the iteration. If the process converges, the general trend is for the mass residual to become smaller as the iteration goes on. Figure 38 shows the convergence behavior of typical computations comparing performance using a previous differential combustion model to the newly developed integral combustion model. The differential model runs into a problem of iteratively oscillating between over- and under-prediction of combustion rates in cells enclosing zones of intense combustion. This problem in the differential model prevents convergence to an acceptable level, about $10^{-9}$, when using the differential model. Recall from the discussion on convergence criteria in Section 3.2 that a gas-phase level of convergence of about $10^{-9}$ is required to produce results that appear identical, when plotted, to results obtained from more highly converged computational runs. Figure 37 shows the clear superiority of the integral combustion model in allowing convergence based on cell mass residual to drop below the limit encountered with the differential model and continue to decrease toward the precision limit of the machine. In these cases computation was performed on a CRAY supercomputer in single precision, which provides between 14 and 15 significant figures. Each iteration takes approximately 0.66 second of computing time and a typical convergent case needs about 400 seconds of supercomputing time to reduce the average mass residual to less than $10^{-9}$. 


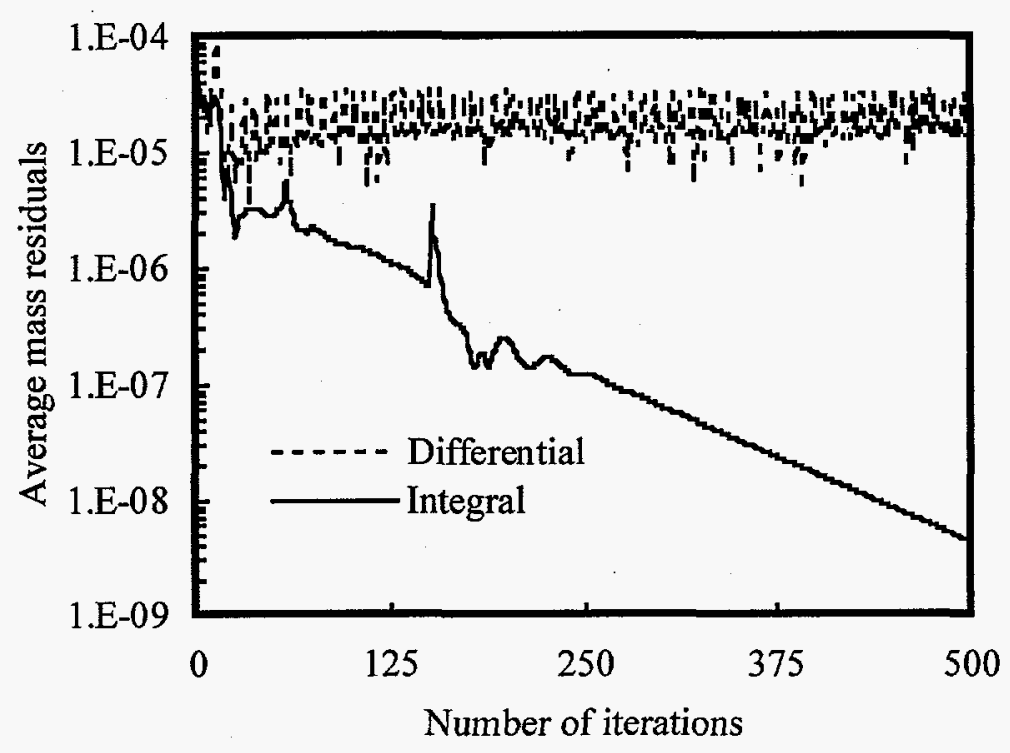

(a) Average

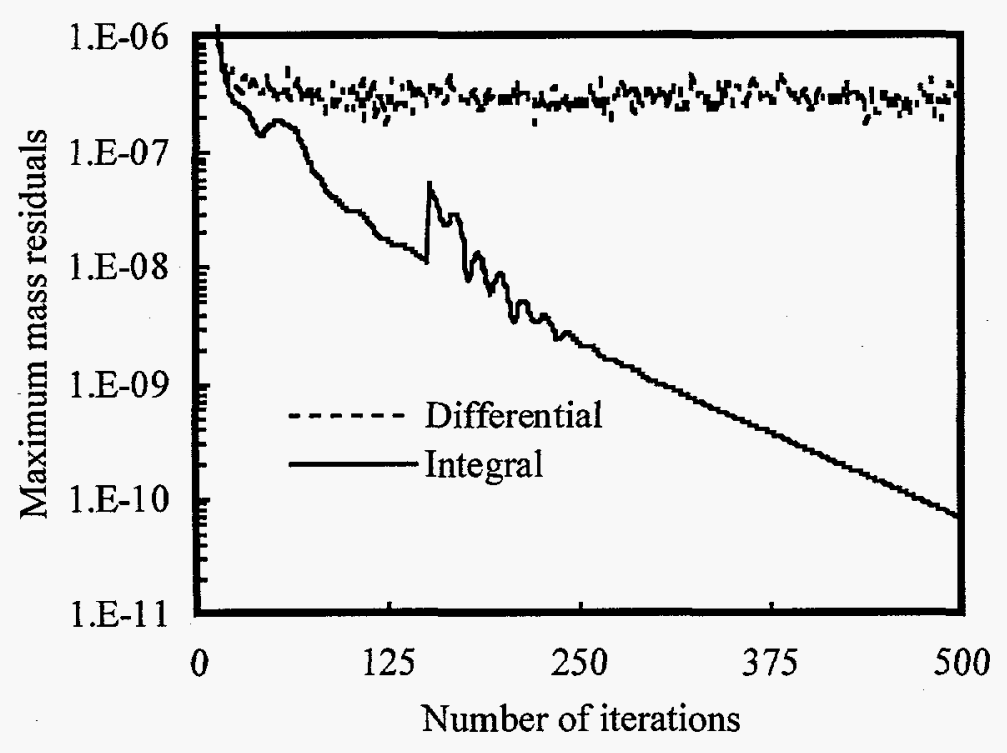

(b) Maximum

Figure 38 Numerical Convergence of Integral and Differential Reaction-Rate Approaches 


\section{IONIZATION OF SEED MATERIAL IN THE SECOND-STAGE COMBUSTOR}

Seed (potassium) particles are injected into the coal gas flow in the deswirl section before entering the second-stage combustor. The seed material evolves in the combustor from particles, to vapor, to ionized atoms. Particle melting/evaporation, vapor ionization, and dispersion of ionized seed vapor are among the important processes that determine the performance of the overall MHD power train system. Incomplete mixing between the seed material and the combustion flow in the combustor makes ionized seed vapor profiles nonuniform before entering an MHD channel. The distortion of the ionized seed vapor profiles can significantly lower the electric conductivity of the gas and subsequently the MHD channel power output. The ICOMFLO computer code was used to investigate the effects of operating conditions on the distortion of the seed material profiles in the second-stage combustor $[25,26]$.

\subsection{Multiphase Reacting Flow System}

Figure 39 shows a 2-D computational domain for the seed/gas flow in the second-stage combustor. The computational domain consists of an inlet (left dashed line), an exit (right dashed line), and two injector slots (arrows) on the top and the bottom side walls. An injection angle $\theta$ is defined as the angle between the bottom wall in the main flow direction and the bottom jet vector. The inlet stream consists of fuel and inert gases and seed particles. Top and bottom jets are oxidizer streams. Both combustion and seed ionization processes take place in the combustor. The combustion process converts fuel and oxidizer to product. The seed ionization process includes particle melting/evaporation and vapor ionization. The exit stream consists of combustion products and ionized seed vapor.

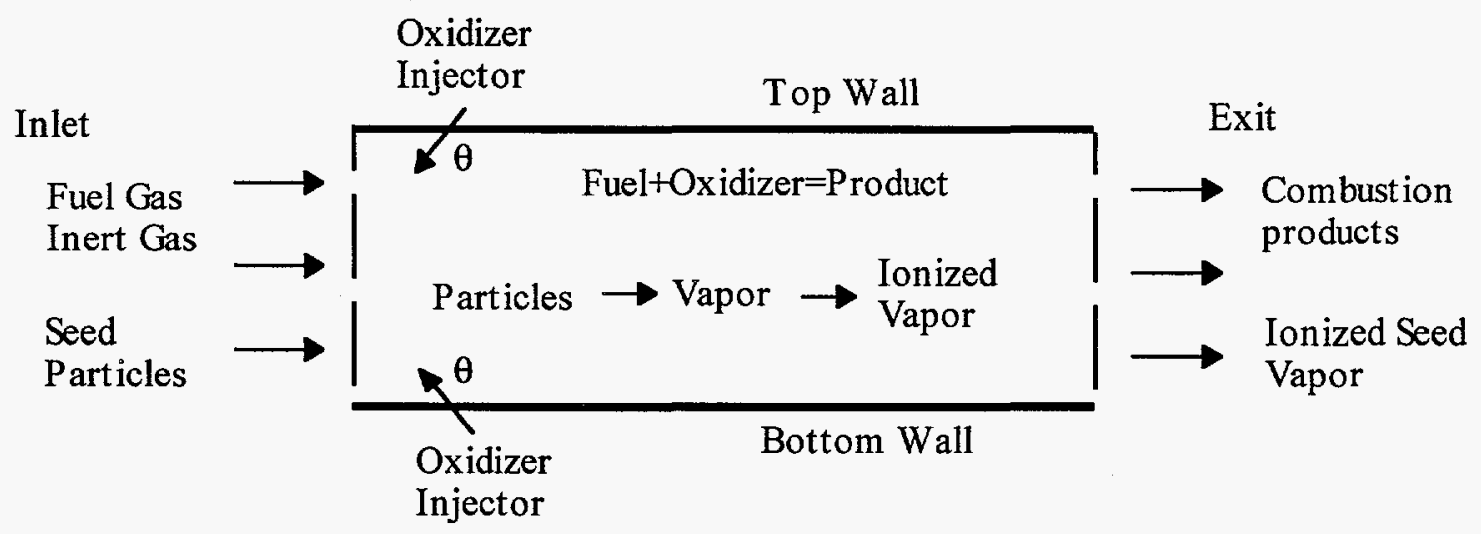

Figure 39 Computational Domain for the Seed/Gas Flow in the Second-Stage Combustor 


\subsection{Phenomenological Submodels for Multiphase Ionization Flows}

The ICOMFLO computer code described in Chapter 4 was enhanced with the integral reaction model and validated for single-phase reacting flows. Although it contained droplet phase submodels, they were not used. The droplet submodels were modified and enhanced so the code could use either droplets or particles as the condensed phase, and the droplet evaporation submodel was enhanced to handle particle melting/evaporation. The enhancements were added to the computer code so it could be used to simulate the multiphase flow in the second-stage combustor, including the following processes: turbulent dispersion of the condensed phase, interfacial momentum and heat transfer, particle melting/evaporation, and ionization of seed vapor.

\subsubsection{Multiphase k- $\varepsilon$ Turbulent Submodel}

The turbulent $\mathrm{k}-\varepsilon$ submodel was modified for multiphase flows to account for turbulent dispersion of particles. The multiphase turbulent transport equations are derived from the singlephase turbulent transport equations by multiplying density terms by void fraction and adding a sink term in the k-equation. The additional sink term in the k-equation accounts for the loss rate of turbulent kinetic energy in the gas phase as this energy is transferred to particles in the turbulent dispersion process. The loss of the turbulent kinetic energy from the gas phase depends on particle size. If the particle size is in a submicron range, the particles can dissipate the turbulent kinetic energy as effectively as the gas and the loss rate is set to the gas-phase kinetic energy dissipation rate. If the particle size is larger than $100 \mu \mathrm{m}$, the loss becomes negligible.

Particle diffusivity also depends on particle size. For the particles larger than $100 \mu \mathrm{m}$, the turbulent fluctuations have little effect, and they can only be transported by their own momentum and interphase transfer of momentum via drag (calculated from the mean slip velocity). If the particle size is in the submicron range, the particles move along with gas flow and have approximately the same turbulent diffusion rate as other gas-phase species in the gas phase. For intermediate particle sizes (from submicron to $100 \mu \mathrm{m}$ ), the turbulent diffusivity of the particle number density equation is defined as,

$$
\Gamma_{\mathrm{ni}}=\mu_{\mathrm{t}} /(0.9 \varphi \rho)
$$

An empirical particle dispersion function $\varphi$ is defined as

$$
\varphi(r)=1+2 r+0.06 r^{2}
$$

where $\mathrm{r}$ is the particle radius.

\subsubsection{Interfacial Drag and Heat Transfer Submodel}

A gas flow is generally driven by a pressure gradient and a particle flow is driven by the drag force from the gas flow. The drag force is caused by the velocity difference between the gas and solid phases (called slip velocity). A slip velocity $u_{\delta}$ is defined as 


$$
u_{\delta}=u-u_{s}
$$

Empirical equations are commonly used to correlate the drag force with the slip velocity. Drag force is a vector defined by two orthogonal components: one in the x-direction and the other in the y-direction. Since the derivation of the two components is the same, only the derivation of the $\mathrm{x}$-component will be presented in the following. For a single particle in the gas, the $\mathrm{x}$-direction drag force $\left(\mathrm{F}_{\mathrm{d}, \mathrm{x}}\right)$ of gas exerted on the particle can be expressed as a function of the slip velocity kinetic energy $\rho u_{\delta}{ }^{2} / 2$, particle cross-sectional area $\pi r^{2}$, and an empirical coefficient $C_{d}$ as shown in Eq. 5.4. The particle drag force is in the same direction as the slip velocity. When the slip velocity is positive, the drag force is positive. When the slip velocity is negative, the drag force is negative.

$$
F_{\mathrm{d}, \mathrm{x}}=\frac{1}{2} \rho\left|\mathrm{u}_{\delta}\right| \mathrm{u}_{\delta} \pi \mathrm{r}^{2} \mathrm{C}_{\mathrm{d}}
$$

Equation 5.5 is a common empirical expression used to define the drag coefficient $\mathrm{C}_{\mathrm{d}}$. The coefficient includes two major effects on the drag force: one is the viscous (or diffusive) effect, represented by a Reynolds number $\mathrm{Re}_{\mathrm{s}}$, Eq. 5.6; the other is the evaporation effect, represented by a transfer number B, Eq. 5.7. In Eq. 5.7, $\mathrm{L}$ is the latent heat of a particle (includes heat necessary to melt and vaporize the particle) and $T_{b}$ is the boiling temperature of a particle after melting. When the particle temperature is less than its boiling temperature, there is no evaporation, the transfer number is zero, and the drag force depends on the viscous effect alone:

$$
C_{d}=\frac{24}{\operatorname{Re}_{s}} \frac{\left(1+0.15 \operatorname{Re}_{s}^{0.687}\right)}{1+B}
$$

where

$$
\begin{aligned}
& \operatorname{Re}_{s}=\frac{2 p\left|u_{\delta}\right| \mathrm{r}}{\mu} \\
& B=\left\{\begin{array}{cc}
\frac{C_{p}\left(T-T_{b}\right)}{L} & \text { if } T>T_{b} \\
0 & \text { if } T \leq T_{b}
\end{array}\right.
\end{aligned}
$$

Similar empirical equations are used to correlate the interfacial heat transfer with the slip velocity. For a single particle in the gas, the heat transfer between the gas and the particle is expressed as

$$
\mathrm{q}_{\mathrm{s}}=2 \pi \mathrm{r} \lambda \mathrm{Nu}_{\mathrm{s}}\left(\mathrm{T}-\mathrm{T}_{\mathrm{s}}\right) \delta\left(\mathrm{T}_{\mathrm{b}}\right)
$$

In Eq. 5.8, $\lambda$ is thermal conductivity of the gas; $\mathrm{Nu}_{\mathrm{s}}$ is a Nusselt number given by an empirical formula, Eq. 5.9; and $\delta\left(T_{b}\right)$ is a step function equal to one for $T_{s}<T_{b}$ and zero otherwise. The Nusselt formula, Eq. 5.8, includes two major effects of the interfacial heat exchange: one is the momentum effect, represented by the Reynolds number $\mathrm{Re}_{\mathrm{s}}$, Eq. 5.6; the other is the diffusivity effect, represented by the Schmidt number Sc, Eq. 5.10. In Eq. 5.10, D is the mass diffusivity of a species. After the particle temperature reaches the boiling temperature, 
further heat transfer from the gas to particles goes into particle evaporation. In this case, the step function $\delta\left(T_{b}\right)$ makes $q_{s}$ zero for $T_{s}$ equal to $T_{b}$.

$$
\begin{aligned}
& \mathrm{Nu}_{\mathrm{s}}=1+0.276 \mathrm{Re}_{\mathrm{s}}^{1 / 2} \mathrm{Sc}^{1 / 3} \\
& \mathrm{Sc}=\frac{\mu}{\rho \mathrm{D}}
\end{aligned}
$$

\subsubsection{Particle Evaporation Submodel}

Particles melt and vaporize in a combustion flow with a temperature higher than the particle boiling point. When heat is received from the surrounding gas, particle temperature increases. When particle temperature reaches the boiling point, particles start to vaporize. The amount of vapor generated in the evaporation process depends on the amount of heat transfer from gas to the particles. The evaporation rate of the particles per unit volume is a source term of the gas continuity equation. Neglecting the interactions between the particles, the evaporation rate can be derived from a single-particle evaporation theory. By adding a Nusselt number $\mathrm{Nu}_{\mathrm{s}}$ to the evaporation correlation, an empirical correlation for vaporization in a flow is derived as

$$
\left(\frac{\mathrm{dm}}{\mathrm{dt}}\right)_{\text {conv }}=4 \pi \mathrm{r}\left(\lambda / \mathrm{C}_{\mathrm{p}}\right) \ln (1+\mathrm{B}) \mathrm{Nu}_{\mathrm{s}}
$$

The total evaporation rate at a point per unit volume of physical space is determined by integrating the product of single-particle evaporation rate and the spray distribution function over particle size space. In addition to mass, evaporation also adds momentum to the gas flow. Also, evaporation reduces particle size and consequently shifts the particle number density distribution toward the small end of the spectrum.

\subsubsection{Gas Ionization Submodel}

An ionized gas can conduct electricity. For MHD power generation, gas ionization is generally acheived by heating the gas in a combustor. The heat of ionization is called the ionization potential. Most combustion gases have a high ionization potential and hence do not ionize thermally in the combustion temperature range of 2000 to $3000 \mathrm{~K}$. However, if an alkali metal such as potassium, which has a lower ionization potential, is added to the gas in the amount of about $1 \%$ by mass, sufficient thermal ionization can be obtained for MHD power generation. The magnitude of the conductivity required for MHD applications is about $10 \mathrm{mho} / \mathrm{m}$.

Thermal ionization of a combustion product gas seeded with potassium, $\mathrm{K}$, in the temperature range of 2000 to $3000 \mathrm{~K}$ proceeds according to

$$
\mathrm{A}+\mathrm{K} \longrightarrow \mathrm{A}+\mathrm{K}^{+}+\mathrm{e}^{-}
$$


where $\mathrm{A}$ is a mixture of combustion gases that do not ionize in the temperature range, $\mathrm{K}^{+}$is the potassium ion, and $\mathrm{e}^{-}$represents the electron. Thermal ionization follows a mass action law like any chemical reaction. The mass action law for thermal ionization is

$$
\frac{\mathrm{n}_{\mathrm{e}} \mathrm{n}_{\mathrm{i}}}{\mathrm{n}_{\mathrm{K}}}=\frac{\left(2 \pi \mathrm{m}_{\mathrm{e}} \mathrm{kT}\right)^{3 / 2}}{\mathrm{~h}^{3}} \exp \left(-\theta_{\mathrm{i}} / \mathrm{T}\right)
$$

where $n_{K}, n_{i}$, and $n_{e}$ are concentrations of potassium atom, ion, and electron, respectively; $m_{e}$ is the electron mass, $9.11 \times 10^{-31} \mathrm{~kg}$; $\mathrm{k}$ is Boltzmann's constant, $1.38 \times 10^{-23} \mathrm{~J} / \mathrm{K} ; \mathrm{h}$ is Planck's constant, $6.62 \times 10^{-34} \mathrm{~J}-\mathrm{s}$; and $\theta_{\mathrm{i}}$ is the characteristic ionization temperature for potassium, $50,200 \mathrm{~K}$. For given conditions of potassium concentration, temperature, and pressure, the electron and ion concentrations can be determined from the mass action law, Eq. 5.13, and the ideal gas law. Eqs. 5.14 and 5.15 state the ideal gas law before and after ionization.

$$
\begin{aligned}
& P V^{\prime}=\left(N_{A}+N_{K}^{\prime}\right) k T \\
& P V=\left(N_{A}+N_{K}+N_{i}+N_{e}\right) k T
\end{aligned}
$$

where $\mathrm{V}^{\prime}$ and $\mathrm{V}$ are volumes before and after ionization and $\mathrm{N}$ is the number of molecules, atoms, or electrons. Note that concentrations after ionization are affected by the volume change of the assumed constant pressure reaction. By defining an electron fraction,

$$
\alpha=\frac{N_{e}}{N_{A}+N_{K}^{\prime}}
$$

and a potassium fraction,

$$
\beta=\frac{N_{K}^{\prime}}{N_{A}+N_{K}^{\prime}}
$$

the ionization mass action law becomes

$$
\frac{\alpha^{2}}{(1+\alpha)(\beta-\alpha)}=\frac{\left(2 \pi \mathrm{m}_{\mathrm{e}} \mathrm{kT}\right)^{3 / 2}}{\mathrm{~h}^{3}} \exp \left(-\theta_{\mathrm{i}} / \mathrm{T}\right)
$$

where $\beta$ is determined from combustion gas composition and potassium seeding. After solving for the electron fraction from Eq. 5.18, combustion gas, potassium atom, ion, and electron concentrations needed for calculating electric conductivity are obtained.

$$
\begin{aligned}
& \mathrm{n}_{\mathrm{A}}=\frac{(1-\beta) \mathrm{P}}{(1+\alpha) \mathrm{kT}} \\
& \mathrm{n}_{\mathrm{K}}=\frac{(\beta-\alpha) \mathrm{P}}{(1+\alpha) \mathrm{kT}} \\
& \mathrm{n}_{\mathrm{e}}=\mathrm{n}_{\mathrm{i}}=\frac{\alpha \mathrm{P}}{(1+\alpha) \mathrm{kT}}
\end{aligned}
$$


Figure 40 shows electron concentrations predicted by Eq. 5.21 for some typical MHD operating conditions: temperature from 2000 to $3000 \mathrm{~K}$; pressure from 1 to $10 \mathrm{~atm}$; and potassium mass fraction from 0 to $2 \%$. In the figure, the solid line represents electron concentration versus temperature calculated at a pressure of $5.7 \mathrm{~atm}$ and a potassium fraction of $1 \%$; the dashed line represents electron concentration versus pressure calculated at a temperature of $2700 \mathrm{~K}$ and a potassium fraction of $1 \%$; and the chain-dot line represents the electron concentration versus potassium fraction calculated at a temperature of $2700 \mathrm{~K}$ and a pressure of $5.7 \mathrm{~atm}$. Predicted electron concentrations show an exponential dependence on temperature, and a linear dependence on the square root of pressure and potassium mass fraction. To validate the gas ionization formula, the predicted electron concentrations were compared against limited experimental data for both potassium and cesium seedings [27-28]. The comparisons showed good agreement. For the temperature, pressure, and potassium seeding conditions listed in Table-9, the predicted electron concentrations are $7.03,4.08$, and $7.70 \times 10^{19} / \mathrm{m}^{3}$, compared against the measurements 7,4 , and $8 \times 10^{19} / \mathrm{m}^{3}$, respectively.

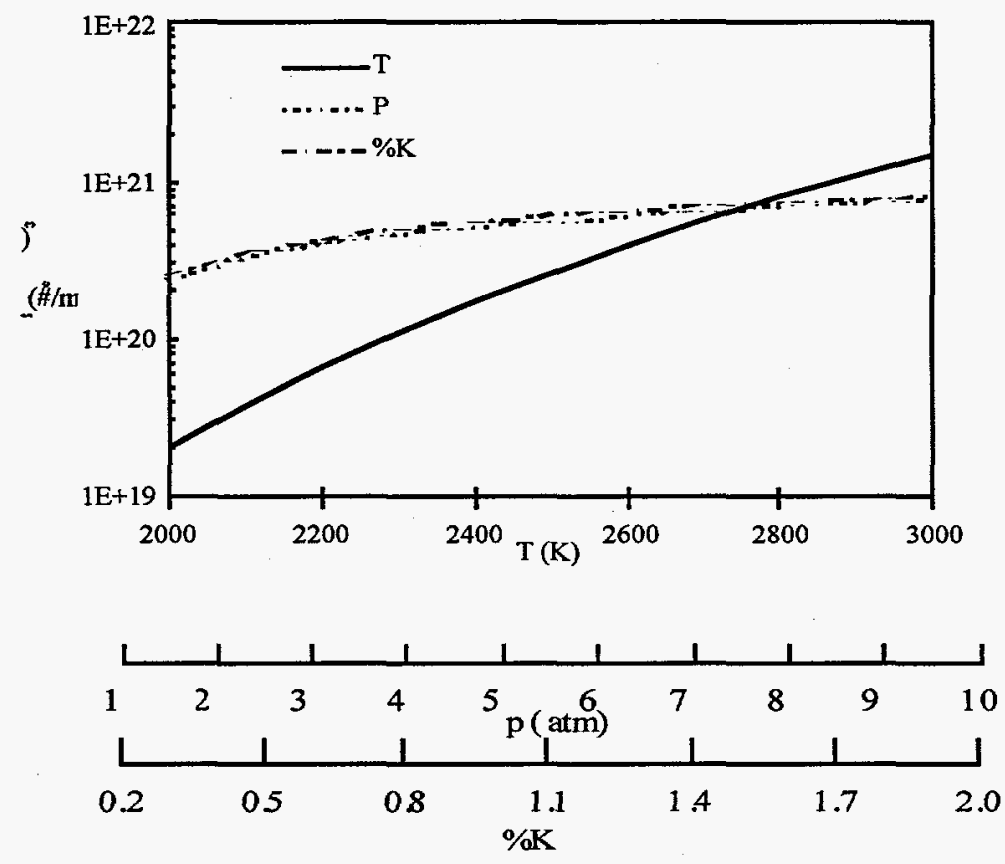

Figure 40 Electron Concentration versus Temperature, Pressure, and Seed Mass Fraction

In combustion product gases the prediction of electron concentration may be complicated by the possibility of the contribution from various radicals. Table 10 summarizes the electron contributions by the species commonly found in coal-fired combustion and some alkali species. The electron concentrations are computed assuming a temperature of $2700 \mathrm{~K}$, a pressure of 5.7 atm, and a species mass fraction of $1 \%$. For alkali species, the electron contributions are 1.26, 5.63 , and $7.65 \times 10^{20}$ electrons $/ \mathrm{m}^{3}$ for $\mathrm{Na}, \mathrm{K}$, and $\mathrm{Cs}$, respectively. For combustion gases, NO is most likely to contribute at about $10^{17}$ electrons $/ \mathrm{m}^{3}$, and other combustion gases can contribute no more than $10^{14}$ electrons $/ \mathrm{m}^{3}$. Under these conditions, only alkali species are important in computing electron concentration for the purpose of calculating gas conductivity. 
Table 9 Comparison of Predicted and Measured Potassium Gas Ionization

\begin{tabular}{|l|r|r|r|}
\hline Temperature $(\mathrm{K})$ & 2780 & 2700 & 2350 \\
\hline Pressure $(\mathrm{atm})$ & 1 & 1 & 1 \\
\hline Potassium concentration $\left(\times 10^{20} / \mathrm{m}^{3}\right)$ & & & \\
\hline measured & 10.5 & 6.33 & 400 \\
\hline predicted & 10.4 & 6.23 & 404 \\
\hline Electron concentration $\left(\times 10^{19} / \mathrm{m}^{3}\right)$ & & & \\
\hline measured & 7.0 & 4.0 & 8.0 \\
\hline predicted & 7.03 & 4.08 & 7.70 \\
\hline Electron collision frequency $\left(\times 10^{11} / \mathrm{s}\right)$ & & & \\
\hline measured & - & 2.03 & - \\
\hline predicted & 2.05 & 2.07 & 2.74 \\
\hline Electric conductivity $(\mathrm{mho} / \mathrm{m})$ & & & \\
\hline measured & - & 5.6 & - \\
\hline predicted & 9.68 & 5.57 & 7.89 \\
\hline
\end{tabular}

Table 10 Electrons Contributed by Species in Flames $(\mathrm{T}=2700 \mathrm{~K}, \mathrm{P}=5.7 \mathrm{~atm}$, species mass fraction $=1 \%)$

\begin{tabular}{|c|c|c|}
\hline Species & $\theta_{\mathrm{i}}(\mathrm{K})$ & $\mathrm{n}_{\mathrm{e}}\left(\# / \mathrm{m}^{3}\right)$ \\
\hline $\mathrm{Cs}$ & 45,200 & $7.65 \times 10^{20}$ \\
\hline $\mathrm{K}$ & 50,200 & $5.63 \times 10^{20}$ \\
\hline $\mathrm{Na}$ & 59,700 & $1.26 \times 10^{20}$ \\
\hline $\mathrm{NO}$ & 107,000 & $9.00 \times 10^{16}$ \\
\hline $\mathrm{O}_{2}$ & 140,000 & $6.10 \times 10^{13}$ \\
\hline $\mathrm{H}_{2} \mathrm{O}$ & 146,000 & $3.27 \times 10^{13}$ \\
\hline $\mathrm{OH}$ & 160,000 & $2.51 \times 10^{12}$ \\
\hline $\mathrm{O}$ & 158,000 & $1.77 \times 10^{12}$ \\
\hline $\mathrm{CO}$ & 164,000 & $9.37 \times 10^{11}$ \\
\hline $\mathrm{CO}_{2}$ & 167,000 & $4.30 \times 10^{11}$ \\
\hline $\mathrm{H}_{2}$ & 181,000 & $1.42 \times 10^{11}$ \\
\hline $\mathrm{N}_{2}$ & 181,000 & $4.02 \times 10^{10}$ \\
\hline
\end{tabular}


The electric conductivity of a gas, like that of a metal, depends on both the number density of free charge carriers and their mobility. The formula for the conductivity has been derived as

$$
\sigma=\frac{\mathrm{n}_{\mathrm{e}} \mathrm{e}^{2}}{\mathrm{~m}_{\mathrm{e}} \mathrm{f}}
$$

where the electron charge e is $1.602 \times 10^{-19} \mathrm{C}$, electron mass $\mathrm{m}_{\mathrm{e}}$ is $9.1 \times 10^{-31} \mathrm{~kg}$, and $\mathrm{f}$ is collision frequency of electrons, which collide with the atoms, molecules, and ions due to random thermal motion. Between an electron and a species (atom, molecule, or ion), the collision frequency simply depends on the thermal velocity of the electron, effective collision cross-sectional area, and species concentration. Assuming combustion gases other than potassium atoms can be treated as one kind of species, collision frequency may be expressed as

$$
\mathrm{f}=\left(\mathrm{n}_{\mathrm{A}} \mathrm{Q}_{\mathrm{A}}+\mathrm{n}_{\mathrm{K}} \mathrm{Q}_{\mathrm{K}}+\mathrm{n}_{\mathrm{i}} \mathrm{Q}_{\mathrm{i}}\right) \mathrm{V}_{\mathrm{e}}
$$

where $\mathrm{Q}$ is effective collision area $\left(\mathrm{m}^{2}\right)$ and $\mathrm{V}_{\mathrm{e}}$ is thermal electron velocity. Assuming a Maxwell distribution of the electron velocity, the mean electron velocity becomes

$$
\mathrm{V}_{\mathrm{e}}=\sqrt{\frac{8 \mathrm{kT}}{\pi \mathrm{m}_{\mathrm{e}}}}
$$

In general, effective collision areas are functions of temperature. For electron-ion collisions, the effective area depends on a Debye length, $\mathrm{p}_{\mathrm{d}}$, and an impact distance, $\mathrm{p}_{\mathrm{i}}$. The Debye length is a measure of how far, on the average, the electrons in a gas will separate from the ions, and the impact distance is the distance of closest approach between two charged particles. The effective electron-ion collision area can be derived theoretically as

$$
\mathrm{Q}_{\mathrm{i}}=28 \pi \mathrm{p}_{\mathrm{i}}^{2} \ln \left(\mathrm{p}_{\mathrm{d}} / \mathrm{p}_{\mathrm{i}}\right)
$$

where

$$
p_{i}=\frac{e^{2}}{12 \pi \varepsilon_{0} k T}
$$

and

$$
p_{d}=\sqrt{\frac{\varepsilon_{0} k T}{n_{e} e^{2}}}
$$

in which $\varepsilon_{0}$ is the vacuum permittivity, $8.8544 \times 10^{-12} \mathrm{C}^{2} / \mathrm{N} / \mathrm{m}^{2}$.

In the temperature range of 2000 to $3000 \mathrm{~K}$, effective collision areas are weak functions of temperature and the simple summation rule of Eq. 5.23 is adequate. Average collision areas of some neutral atoms and molecules are available in the literature; for example, the effective collision areas of potassium, air, water vapor, and carbon dioxide are $400,8,75$, and $15 \times$ $10^{-20} \mathrm{~m}^{2}$, respectively. To simplify the calculation, the electron-potassium collision area is assumed to be constant, while an empirical correlation is developed for the electron-gas collision 
area. The $\mathrm{Q}_{\mathrm{A}}$ correlation is determined by matching collision frequencies calculated by Eq. 5.23 with those obtained by a more complicated but more accurate rule proposed by Frost [29]:

$$
\begin{aligned}
& \mathrm{Q}_{\mathrm{K}}=4 \times 10^{-18} \mathrm{~m}^{2} \\
& \mathrm{Q}_{\mathrm{A}}=23(2800 / \mathrm{T})^{0.15} \times 10^{-20} \mathrm{~m}^{2}
\end{aligned}
$$

In the temperature range of 2000 to $3000 \mathrm{~K}$, collision frequency consists primarily of electron-gas collision; only a few percent of this frequency is a contribution from electronpotassium and electron-ion collisions. The electron-ion collisions are important when the degree of ionization is high. The electron-potassium collisions are significant when the seeding is high.

Figure 41 shows collision frequency versus temperature, pressure, and seed mass fraction. Figure 42 shows electric conductivity versus temperature, pressure, and seed mass fraction. Reference ionization conditions for the calculations shown in these two figures are the temperature, $2700 \mathrm{~K}$, the pressure, $5.7 \mathrm{~atm}$; and the seed mass fraction, 1\%. For each of the curves plotted in the figure, one of these conditions is varied while the other two are held constant at the reference condition. Collision frequency is a strong function of pressure and temperature and a weak function of seed mass fraction. Collision frequency decreases as the temperature increases, and increases as the pressure and seed mass fraction increase. The conductivity depends strongly on these three parameters. In a rough approximation, it increases exponentially with temperature, decreases with the square root of pressure, and increases with the square root of seed mass fraction. At the reference ionization conditions, collision frequency is $1.3 \times 10^{12} \mathrm{~Hz}$ and electric conductivity is $12 \mathrm{mho} / \mathrm{m}$.

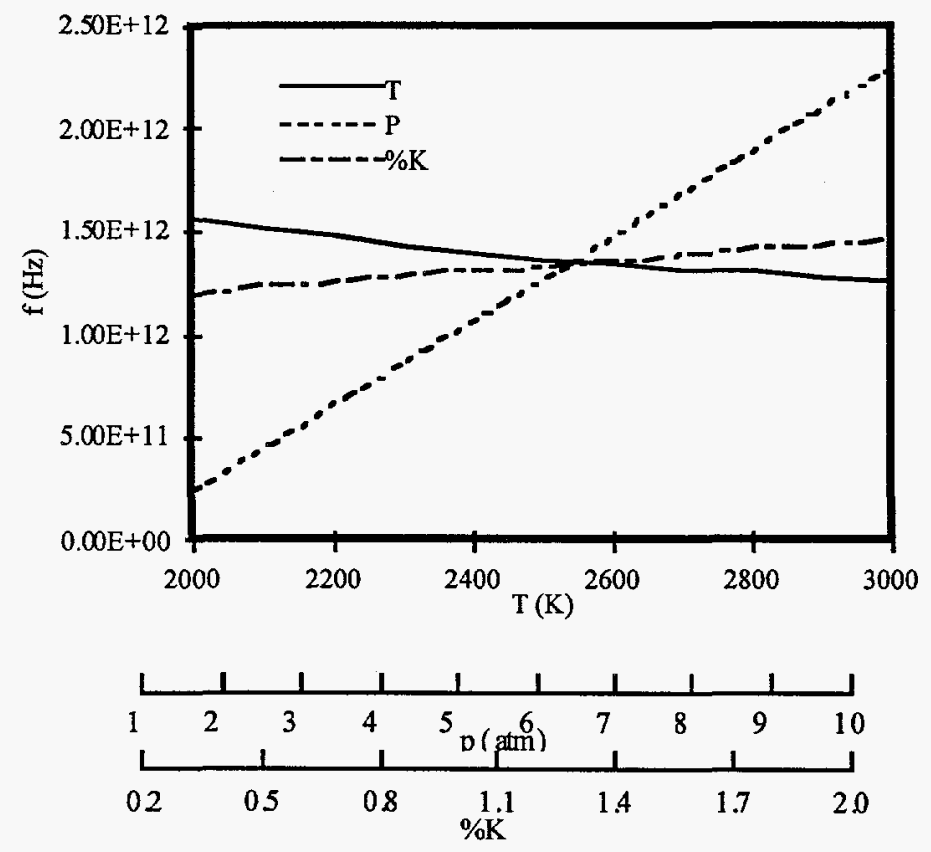

Figure 41 Collision Frequency versus Temperature, Pressure, and Seed Mass Fraction 


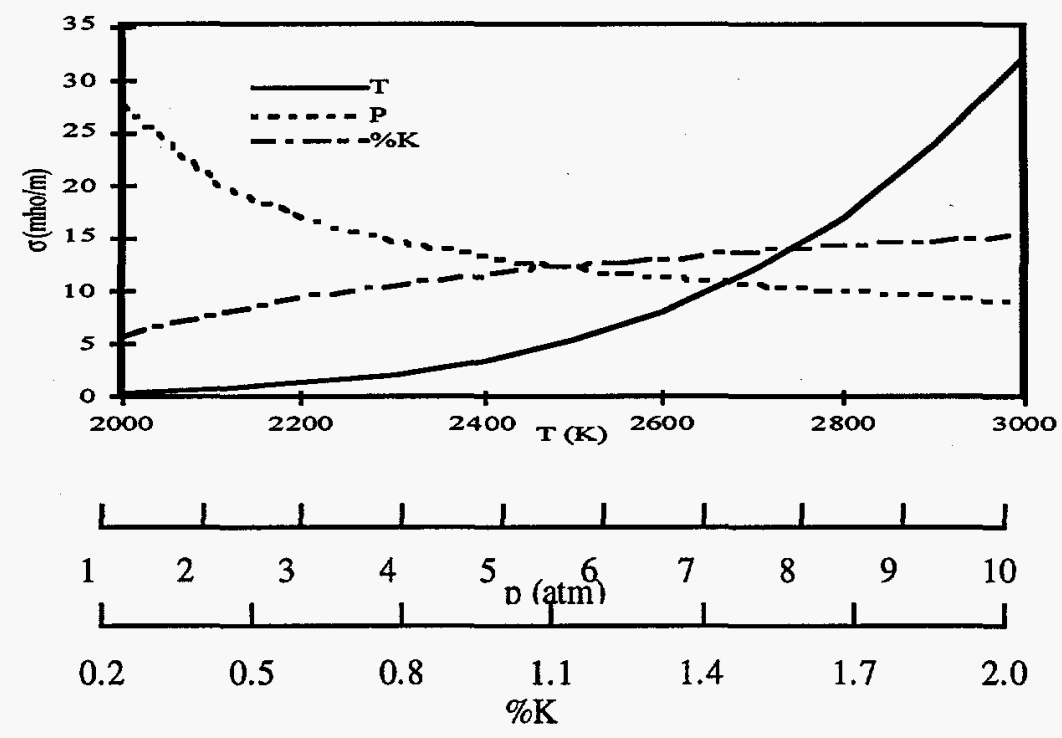

Figure 42 Electric Conductivity versus Temperature, Pressure, and Seed Mass Fraction

\subsection{Particle-Jet Interactions and Seed Vaporization}

A parametric study was performed to study particle-jet interactions in reacting flows, and to investigate the effect of potassium seed injection conditions on wall deposition and vapor dispersion. The study includes two parts: one assumes particles do not vaporize and the other assumes particles vaporize at a fixed temperature with a combined heat of fusion and vaporization. The motivation for studying nonvaporizing particles was that a percentage of slag particles do pass from the first stage combustor into the second-stage. The interest in slag particles arises because completion of combustion in the second-stage combustor requires counter flow injection at the oxidizer jets. This counter flow is of sufficient magnitude to stagnate through drag the streamwise momentum of a portion of particles which are not near the chamber center. Such stagnated particles may deposit on the combustor walls in front of the oxidizer jets, and if the deposition rate is too high, either the oxidizer jet nozzles could clog or the operating time before the combustor would have to be removed and cleaned out could be too short. Common flow conditions used for the study are summarized in Table 11.

\subsubsection{Flow Patterns of Nonvaporizing Particles}

For reacting flows laden with single-sized nonvaporizing particles, several cases were computed. Particle flow patterns are found to be different than those of the gas flow. Particles entering the combustor are pushed either to the combustor center to move around the vortices created by counter flow oxidizer jets or to the side walls, via interfacial drag. For very small particles, i.e., particle diameter less than $5 \mu \mathrm{m}$, wall deposition is negligible and most particles flow around the vortices to the combustor center. 
Table 11 General Multiphase Flow Conditions in the Second-Stage Combustor

\begin{tabular}{|c|c|}
\hline Combustor aspect ratio (L/D) & 3.84 \\
\hline Inlet Temperature $(\mathrm{K})$ & 1974 \\
\hline Jet Temperature (K) & 300 \\
\hline Jet Location $\left(\mathrm{L}_{\mathrm{i}} / \mathrm{D}\right)$ & 0.66 \\
\hline Jet velocity/ $\mathrm{U}_{0}$ & 13.7 \\
\hline Pressure (atm) & 5.7 \\
\hline Overall Stoichiometric ratio & 1.0 \\
\hline Inlet Fuel Mass Fraction & 0.38 \\
\hline Jet Oxidizer Mass Fraction & 1.0 \\
\hline Particle Boiling Temperature $(\mathrm{K})$ & 1594 \\
\hline Inlet Particle Velocity $(\mathrm{m} / \mathrm{s})$ & 25 \\
\hline Bulk Seed Mass Fraction (\%) & 1 \\
\hline
\end{tabular}

For larger particles, wall deposition can be significant. Figure 43 compares particle wall deposition rates for three particle diameters: 30,60 , and $120 \mu \mathrm{m}$. Most significant particle deposition is found near particle flow stagnation points, about half-way between the inlet and the jet location. Peak deposition rates at $\mathrm{X} / \mathrm{D}=0.3$ are 12,5 , and $2 \mathrm{~mm} / \mathrm{hr}$ for the $30-, 60-$, and $120-\mu \mathrm{m}$ cases, respectively, under the $1 \%$ particle mass loading condition. Note that at the combustor inlet, particles are assumed to flow parallel to the walls. Particle deposition rates downstream of the jets are small because particles are pushed to chamber center as the main flow passes the jets and dispersion back out from combustor center beyond the jets is relatively slow, being primarily a diffusive process.

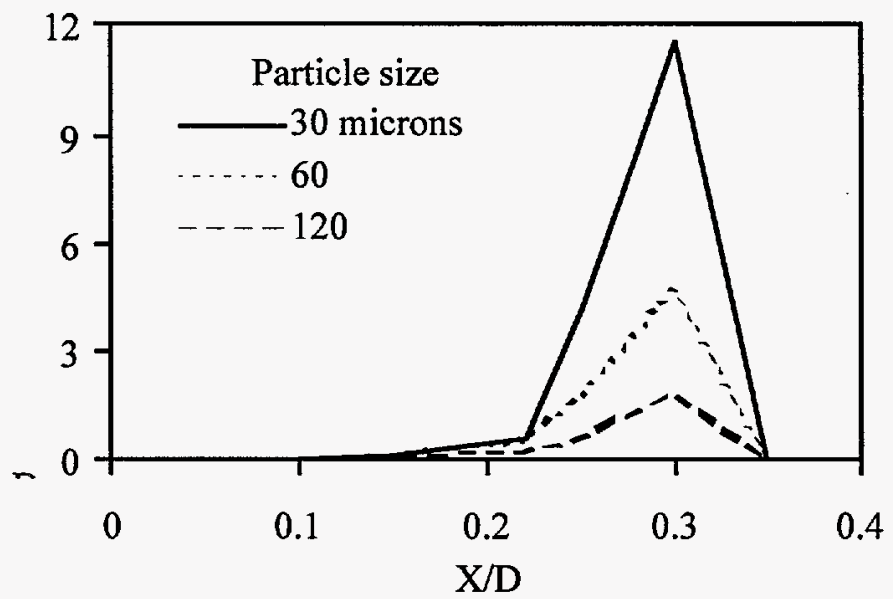

Figure 43 Particle Deposition Rate on Combustor Wall (No Evaporation) 
Figures 44 and 45 show flow patterns of multiple-sized particles in the combustor. This step is essential before simulating particle evaporation in reacting flows, because particle size changes during evaporation. For simple illustrations, three particle sizes of 20,60 , and $100 \mu \mathrm{m}$ are chosen and their inlet number densities are assumed to be $2.08 \times 10^{6}, 8.91 \times 10^{7}$, and $8.87 \times 10^{6}$ particles $/ \mathrm{m}^{3}$, respectively.

In Figure $44 a$, total number density of all particle sizes is shown. The normalized number density value is $10^{8}$ particles $/ \mathrm{m}^{3}$. Particles are pushed to the center portion of the combustor due to the screening effect of the oxidizer jets. Figure $44 \mathrm{~b}$ shows local normalized average particle size. The reference particle size used for normalization is the average inlet particle size, $34 \mu \mathrm{m}$. The variation of local average particle size over the interior of the combustor clearly indicates that particles of different sizes have different flow patterns. Figure 45 shows number density plots of each particle size group. Smaller particles follow the main gas flow more closely than larger particles. Thus, smaller particles turn more rapidly toward the center following the main gas flow as it accelerates into the center in order to pass the opposing jet flows. Further, once past the jets, smaller particles begin to spread back out over the chamber cross section more rapidly than larger particles. This more rapid spreading of smaller particles in the downstream is due both to a larger turbulent diffusion coefficient and to a more rapid drag response to the gas flow.

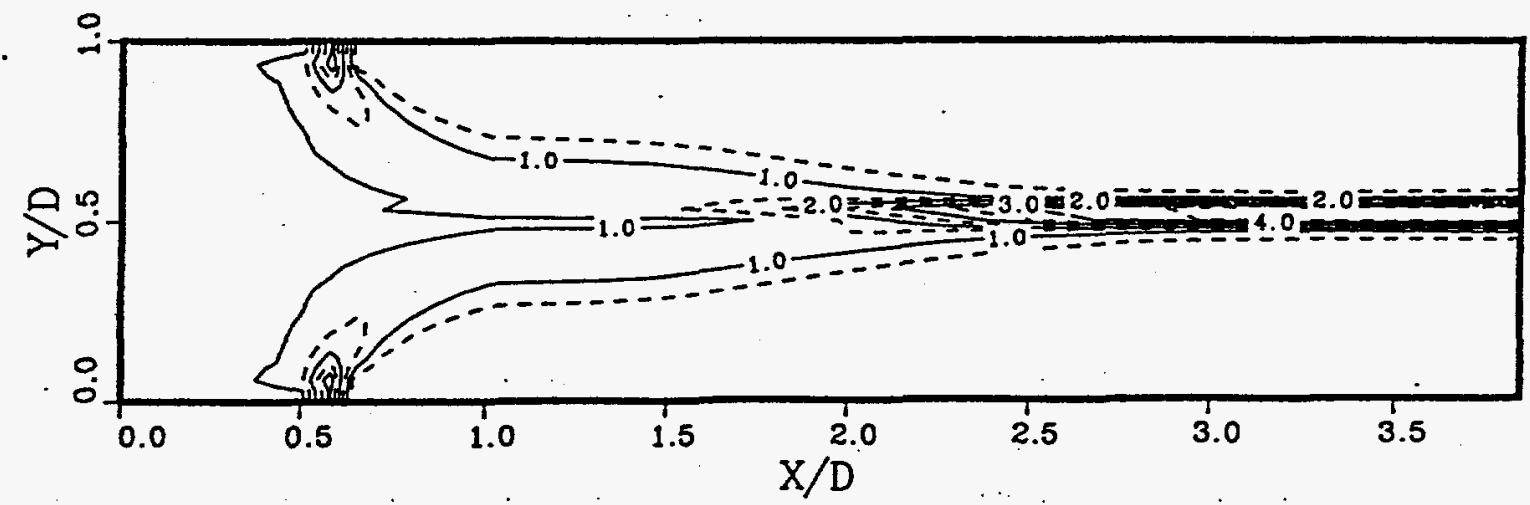

(a) Particle Number Density (All Sizes)

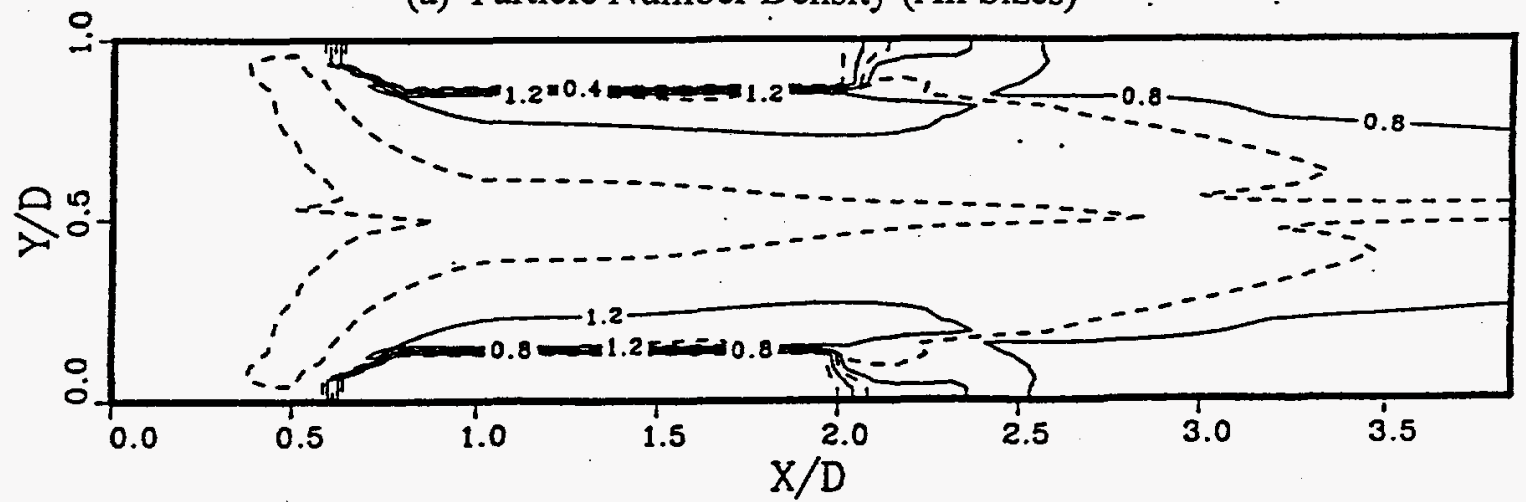

(b) Average Particle Size (Normalized)

Figure 44 Distribution of Nonvaporizing Particles in the Combustor 


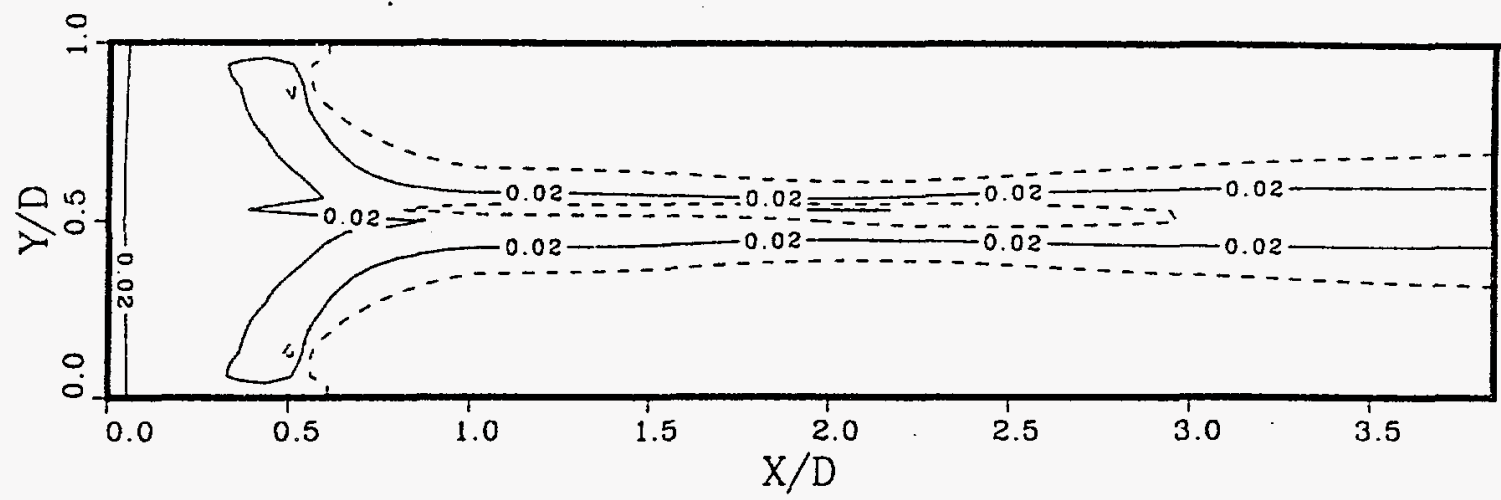

(a) $20 \mu \mathrm{m}$

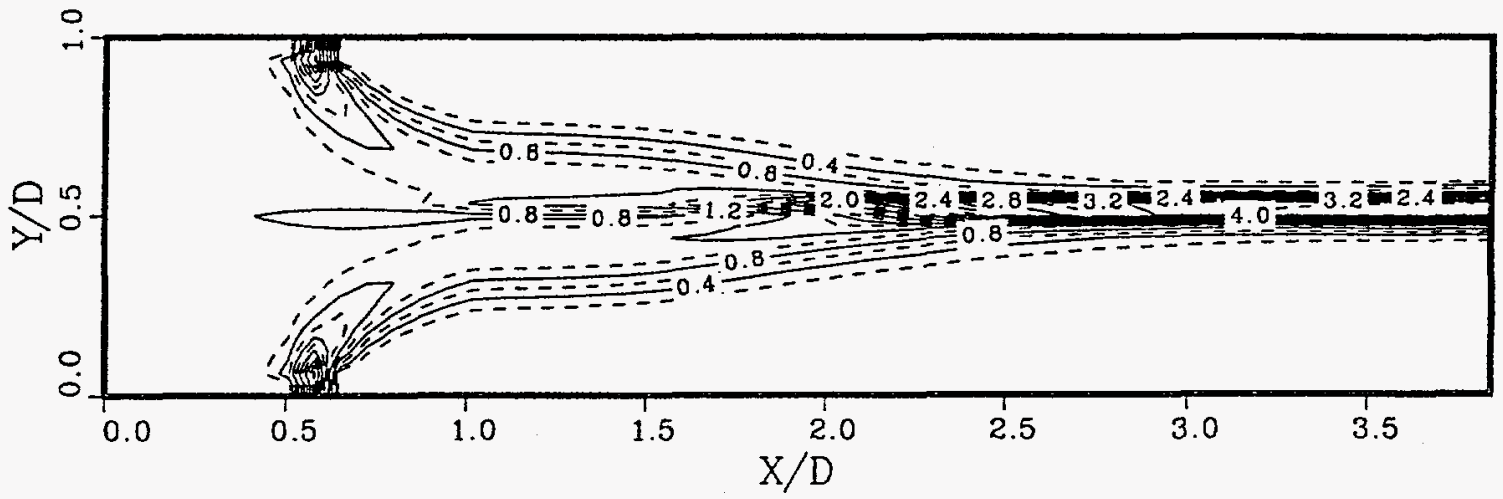

(b) $60 \mu \mathrm{m}$

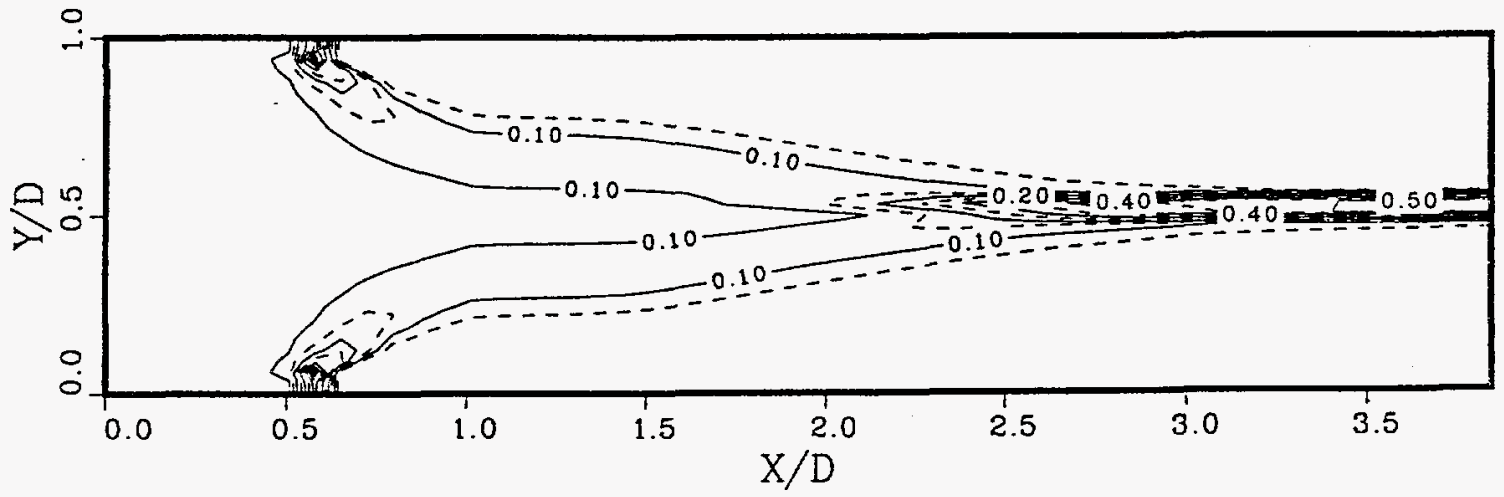

(c) $100 \mu \mathrm{m}$ 

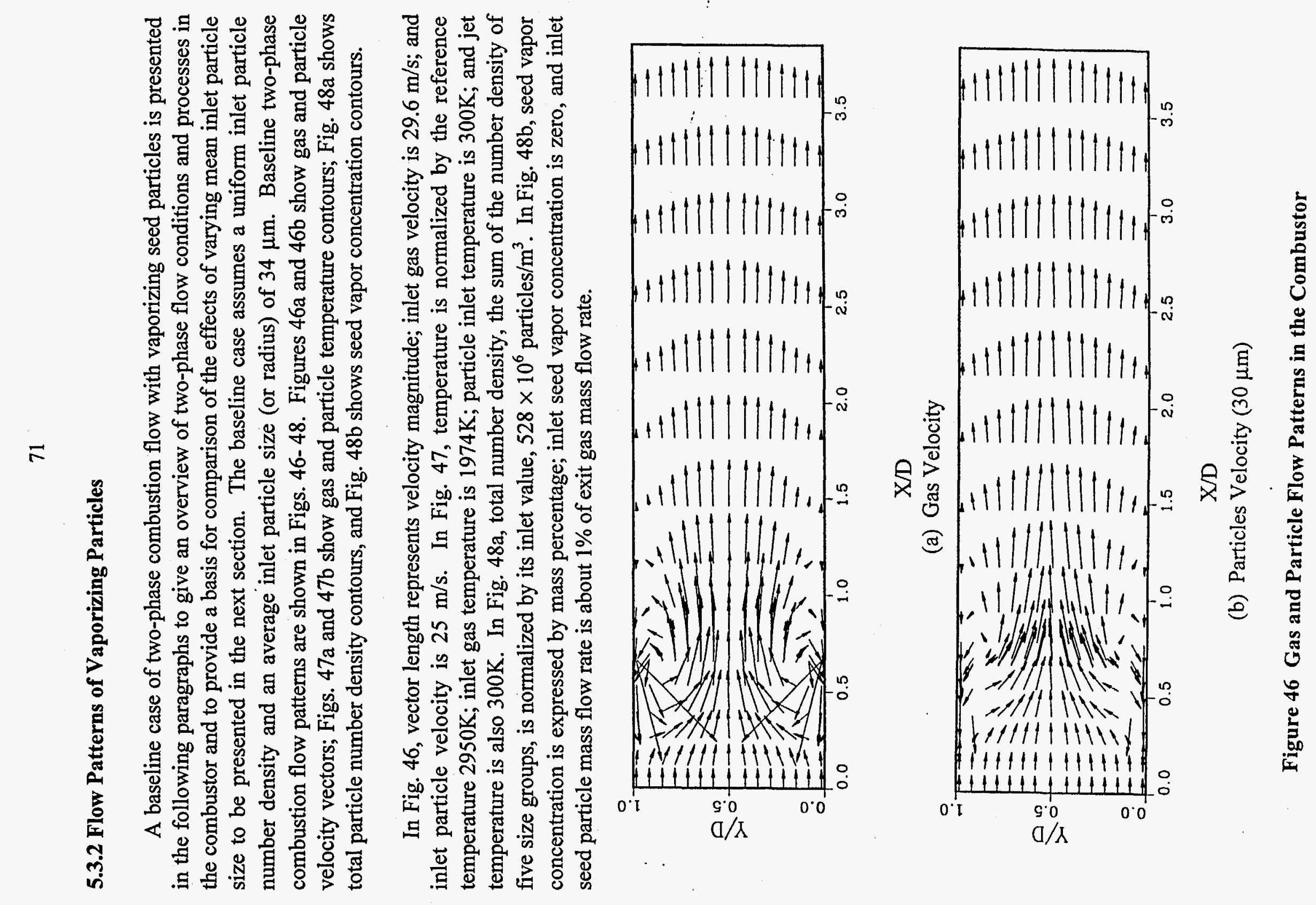
With 130-degree counter-flow injection, intense mixing occurs upstream of the injectors at $\mathrm{X} / \mathrm{D}=0.66$, and the flame is established in this intense mixing zone (Fig. 47a). Because the jets penetrate deeply into the main flow, large vortices form behind the jets (Fig. 46a), and the flame follows the vortex boundary from the upstream, where fuel and oxidizer first meet, toward the chamber center as the oxidizer jets are turned downstream. The flame then continues to develop as a diffusion flame in the mixing layer between fuel and oxidizer in the downstream. Computed results show that the fluid temperature, the fuel concentration, and the oxidizer concentration at the exit are approaching their respective equilibrium values.

In Fig. 46, particle and gas show different flow patterns because of slip velocities. Slip velocity between gas and particles diminishes as the gas flow develops and particles move downstream in the combustor. Because smaller particles react more quickly to the effects of drag, smaller particles have smaller slip velocities. For particles smaller than $5 \mu \mathrm{m}$, slip velocity is negligible. In Fig. 47, particle temperature lags gas temperature as particles are heated up by the gas. Slip temperature (or temperature difference) between gas and particles becomes smaller as particles move downstream in the combustor, until the particle temperature reaches boiling temperature. The smaller the particle, the faster the particle temperature reaches boiling temperature. For particles smaller than $5 \mu \mathrm{m}$, particle temperature reaches boiling temperature almost immediately. Figure 48a shows that some particles are pushed by the oxidizer jets to the combustor center before they are vaporized; some particles impinge on the wall upstream of the oxidizer jet openings; and some particles escape the combustor. At boiling temperature, $\mathrm{T}_{b}=$ $1595 \mathrm{~K}$, particles vaporize at a rate depending on the surrounding gas temperature and slip velocity. Figure $48 \mathrm{~b}$ shows that seed vapor is formed primarily near the combustor center and is gradually diffused to the side walls as the gas flows downstream.
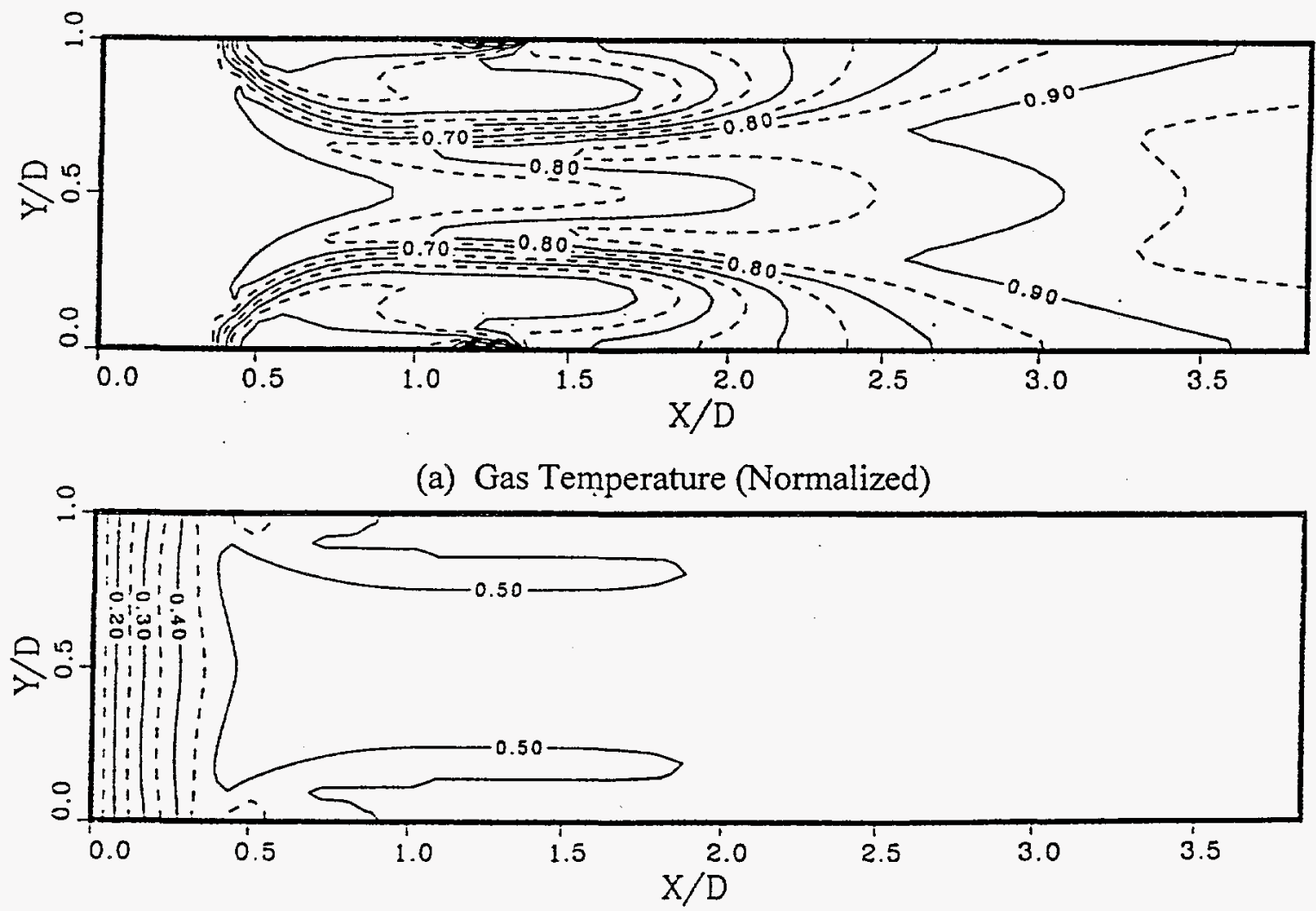

(b) Particle Temperature $(30 \mu \mathrm{m})$

Figure 47 Gas and Particle Temperature Distributions in the Combustor 
The predicted combustion flow patterns for various oxidizer jet angles have been computed and compared. Oxidizer jet angle is found to have a large effect on combustion performance as well as onparticle evaporation. Predicted flow patterns of a 50-degree oxidizer jet injection case contrast sharply with those of 130-degree oxidizer jet injection, described above. For 50-degree injection the oxidizer jets do not penetrate significantly into the main flow but rather are rapidly turned into the downstream, forming a thick high-gradient region near the walls. This flow configuration creates a nearly pure diffusion flame with a relatively low rate of mixing and combustion compared to the 130-degree injection case. Clearly, the change of combustion flow patterns affects the combustion performance, especially, the uniformity of temperature profile at the exit plane (or exit thermal mixedness), which has great influence on the particle vaporization and vapor dispersion. The dependence of exit seed vapor mixedness on jet angle for a two-phase combustion flow is found to be similar to those of thermal mixedness for gas combustion flow and fluid mixedness for nonreacting flow. A large increase in particle vaporization occurs when going from co-flow injection to counter-flow injection. The angle range for most effective particle vaporization and vapor dispersion is approximately centered around 130 degrees.

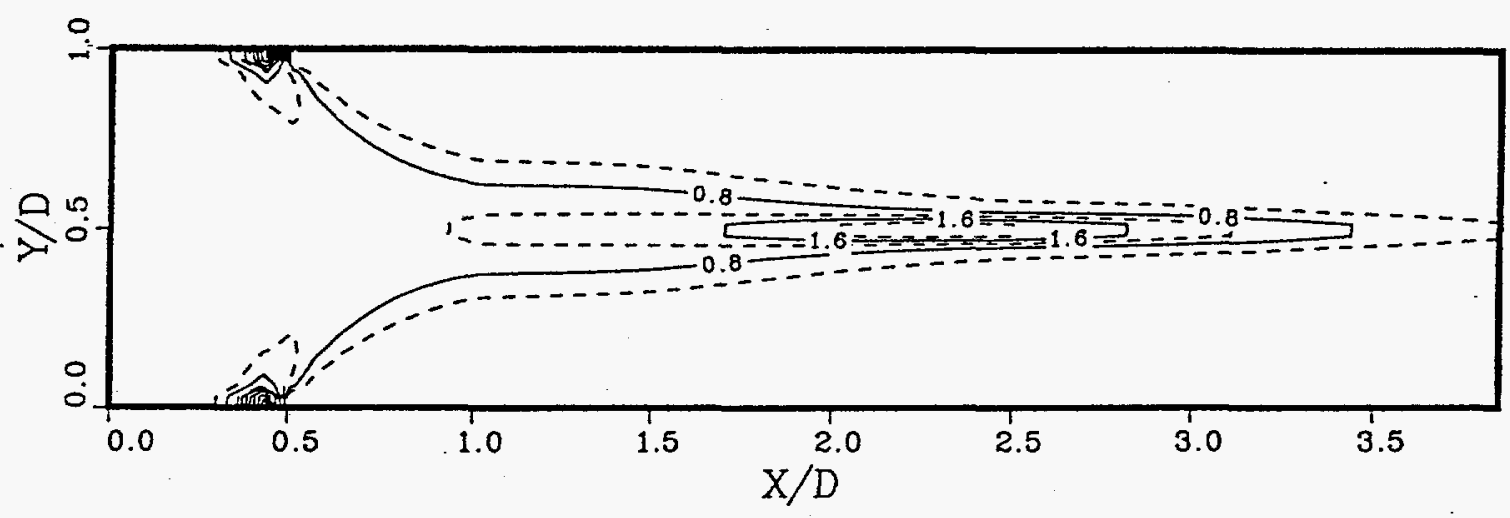

(a) Particle Number Density (all sizes)

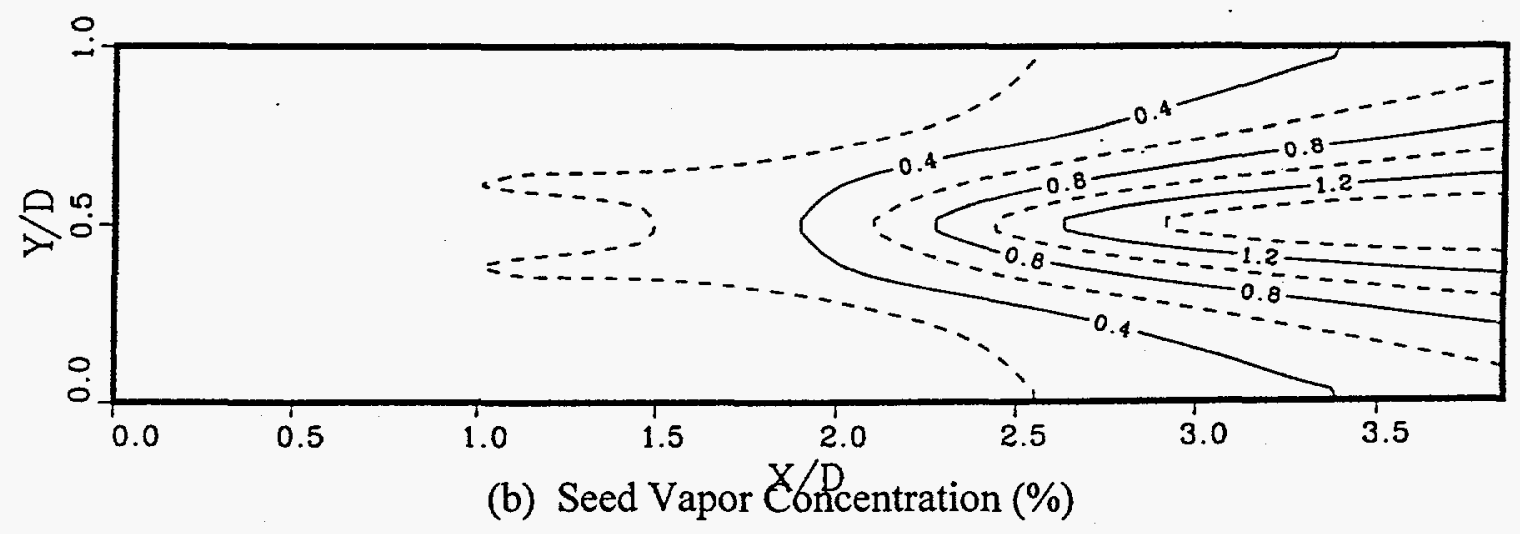

Figure 48 Distributions of Seed Particles and Vapor in the Combustor 


\subsubsection{Effect of Particle Size on Seed Vapor Dispersion}

Particle size has a significant effect on overall particle evaporation (larger particles take longer to vaporize) with consequent impact on seed vapor mixing and uniformity of the seed vapor profile at the combustor exit. Computations were performed for four different inlet mean particle radii in the range 8.5 to $68 \mu \mathrm{m}$. One set of computations assumed a uniform distribution of particles across the chamber inlet. A second set of computations assumed a normal distribution with the peak of the inlet particle number density occurring at the chamber midplane.

Results presented in the next few paragraphs are for cases of uniform inlet number density distribution. The change of total number density (the sum of number density over the particle size spectrum at a point) along a line of grid points adjacent to the chamber centerline, which is hereafter referred to as the centerline, is shown in Fig. 49 for various inlet mean particle radiuses. The injected particles are assumed to be cold relative to the incoming gas stream from the first stage combustor. Before vaporizing, the particles must be heated by the surrounding gas. This prevaporization heating takes longer for large particles than for small particles, and therefore number densities for cases with larger size particles remain higher in the downstream of the chamber. A second process affecting particle number density at chamber center is the screening effect of the jets. The main flow is forced toward chamber center in order to pass the sidewall oxidizer jets, and particles are pushed into the chamber center in this process through drag effects. For the smallest particle case (mean radius $8.5 \mu \mathrm{m}$ ) most of the vaporization takes place upstream of the jets (at $\mathrm{X} / \mathrm{D}=0.66$ ), and therefore, the total center line number density for this case drops rapidly and continuously due to vaporization as the particle flow moves downstream from the inlet. For the $34-\mu \mathrm{m}$ radius case, a large buildup of particle number density in the chamber center is seen in Fig. 49 due to the screening effect of the jets. Larger particles take significantly longer time to heat up and to vaporize than smaller particles. As shown in Fig. 49 for the $34-\mu \mathrm{m}$ radius case, centerline number density remains above $40 \%$ of the inlet value all the way to the exit plane. Results show that for the larger inlet mean particle radius cases $(34 \mu \mathrm{m}$ and above), significant numbers of particles escape the chamber before vaporization has been completed near the chamber center.

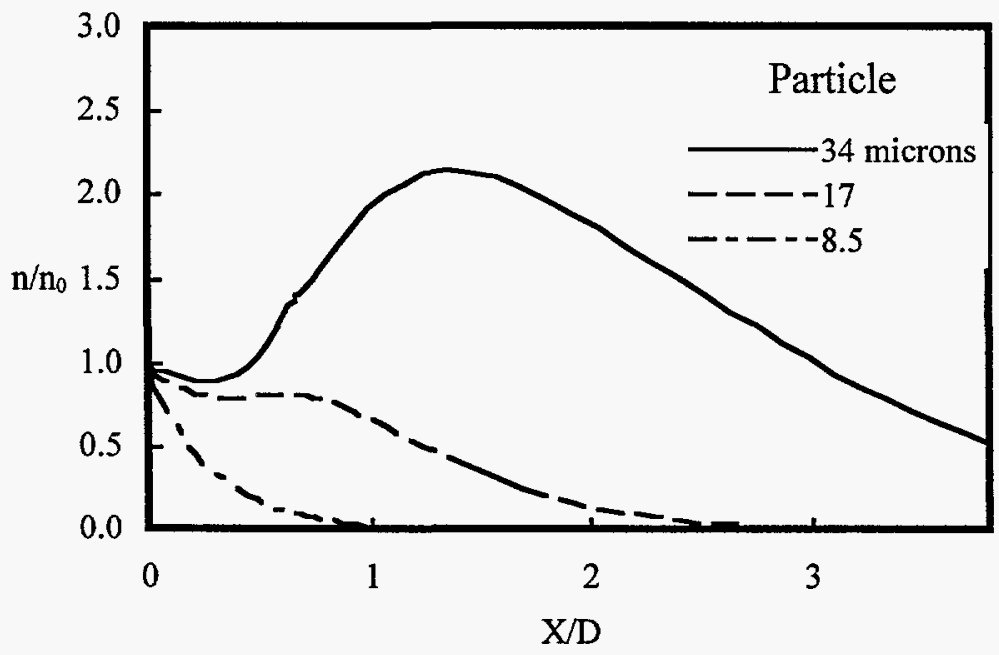

Figure 49 Particle Number Densities of Various Size Groups in the Combustor $(\mathrm{y} / \mathrm{D}=\mathbf{0 . 5})$ 
Of course, number density is not a measure of the mass fraction of the seed particles that has vaporized at the chamber exit plane; as particles vaporize they decrease in size, but they still exist and contribute to the total number density until they have vaporized completely and their radius becomes zero. The fraction of injected particle mass vaporized at the combustor exit for the $34-\mu \mathrm{m}$ case is about $90 \%$; for the cases of smaller particles $(17-$ and $8.5-\mu \mathrm{m}$ mean inlet radius), over $98 \%$ was vaporized by chamber exit, while for the largest mean inlet radius case tested $(68 \mu \mathrm{m})$, the fraction of inlet particle mass vaporized dropped to about $40 \%$.

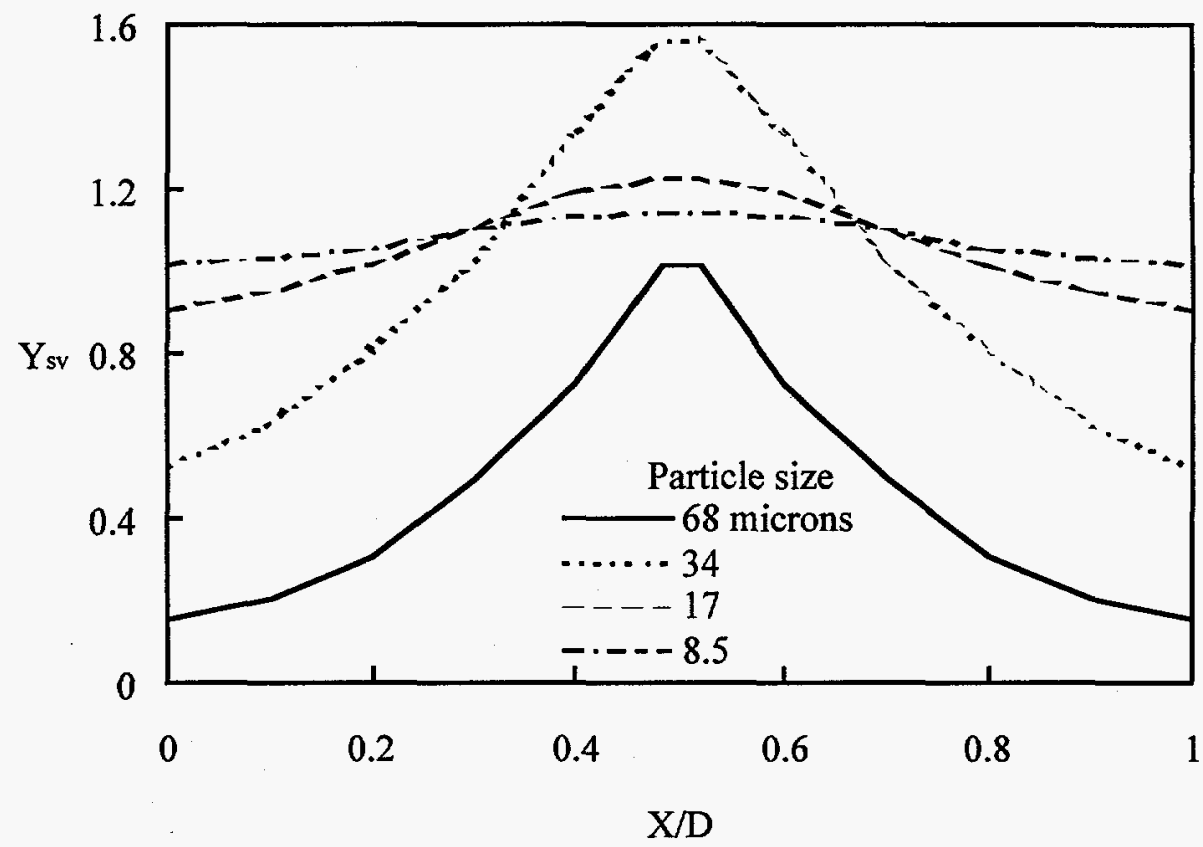

Figure 50 Seed Vapor Concentration Profiles at the Combustor Exit for Various Mean Particle Sizes

Both the extent of vaporization and the location of vaporization vary with mean inlet particle size over the range of particle sizes tested. As previously noted, particles that have not vaporized upstream of the jets are pushed via interfacial drag to the chamber center. Due to the presence of recirculation zones behind the jets, the spreading of the main flow back across the chamber in the downstream of the jet location is gradual. As a consequence, the component of particle drag away from the center line in the downstream of the jets is very small, and particles tend to remain concentrated near the chamber centerline in the region downstream of the jets. Therefore, when vaporization of larger particles occurs downstream of the jets due to prevaporization heating delay and longer vaporization time, seed vapor is primarily deposited near the chamber center in that downstream region. In the MHD application, a uniform distribution of seed vapor is desirable, and delayed vaporization for larger seed particle sizes makes the exit distribution of seed vapor much less uniform. This result is shown is Fig. 50, where exit seed vapor concentration profiles are plotted. For the cases with mean inlet particle radii of 8.5 and $17 \mu \mathrm{m}$, the seed vapor distribution at the exit is fairly uniform. For the $8.5-\mu \mathrm{m}$ case, much of the vaporization occurs upstream of the jets. In the $17-\mu \mathrm{m}$ case, a large portion of 
the vaporization occurs downstream of the jet inlet plane, and therefore near the chamber center; however, most of the vaporization still occurs far enough upstream of the chamber exit for the vapor deposited near the chamber center to spread out across the chamber via diffusion and turbulent transport, giving a relatively uniform seed vapor distribution at the chamber exit plane. For the case of 34- $\mu \mathrm{m}$ mean inlet particle radius, much of the vaporization occurs too far into the downstream of the chamber for the spreading effects of diffusion and turbulence to have much impact by the time the flow reaches the chamber exit, and therefore the seed vapor distribution for the $34-\mu \mathrm{m}$ inlet mean particle size case has a pronounced peak in the chamber center $(\mathrm{Y} / \mathrm{D}=0.5)$, Fig. 50 .

The presence of vaporizing particles in the flow may affect the combustion progress through heat removed from the gas phase for vaporization, dilution of reactants with seed vapor, slight changes in hydrodynamics due to momentum transfer via interfacial drag, and turbulent kinetic energy removed from the gas phase also due to interfacial drag. In the cases tested, which all used a particle loading of approximately $1 \%$ by mass, the effect of inlet mean particle size on combustion progress, measured by the change of center line fuel concentration, was small. The peak gas temperature (near $3000 \mathrm{~K}$ ) drops about $50 \mathrm{~K}$ due to the presence of particles.

A second set of cases was computed with a normal distribution of inlet number density. This distribution concentrates particles in the chamber center at the inlet and simulates an injector source of particles in the near upstream of the inlet plane. As noted in the discussion of cases with a uniform particle distribution at the inlet, the screening effect of the oxidizer jets at $\mathrm{X} / \mathrm{D}=0.66$ tends to push the particle flow into the chamber center. The difference between a uniform inlet distribution of particles and a normal distribution with number density peak in the center is primarily a consequence of processes upstream of the jets. The main difference in vaporization and seed vapor distribution occurring between the uniform and normal distribution of particles at the inlet is an increase in the portion of vaporization occurring near the centerline, primarily for the cases of smaller inlet mean particle size. Because this increase in near center deposition of seed vapor occurs primarily upstream of the jets, sufficient length of chamber remains for much of this vapor to spread out over the chamber cross section as the flow proceeds downstream. Consequently, seed vapor distributions are slightly, but not dramatically, less uniform at the chamber exit for the normal inlet distribution cases than for the uniform inlet distribution cases, and the effect is more pronounced for the smaller inlet mean particle sizes.

The computation of each case also uses a normal distribution of particle sizes about the inlet mean particle size to represent the inlet particle size spectrum. For purposes of discrete computation on a digital computer, the size spectrum is divided into a number of particle size bands or groups. The change of particle number density along the center line for the case with an inlet mean particle radius of $34 \mu \mathrm{m}$ is shown in Fig. 51 for several of the individual size 
groups. The vaporization process in a particular size band decreases the number density in that size group and increases the number density in the next smaller size group. The screening effect of the jets also tends to increase number density in all size groups as particles are pushed to the center.

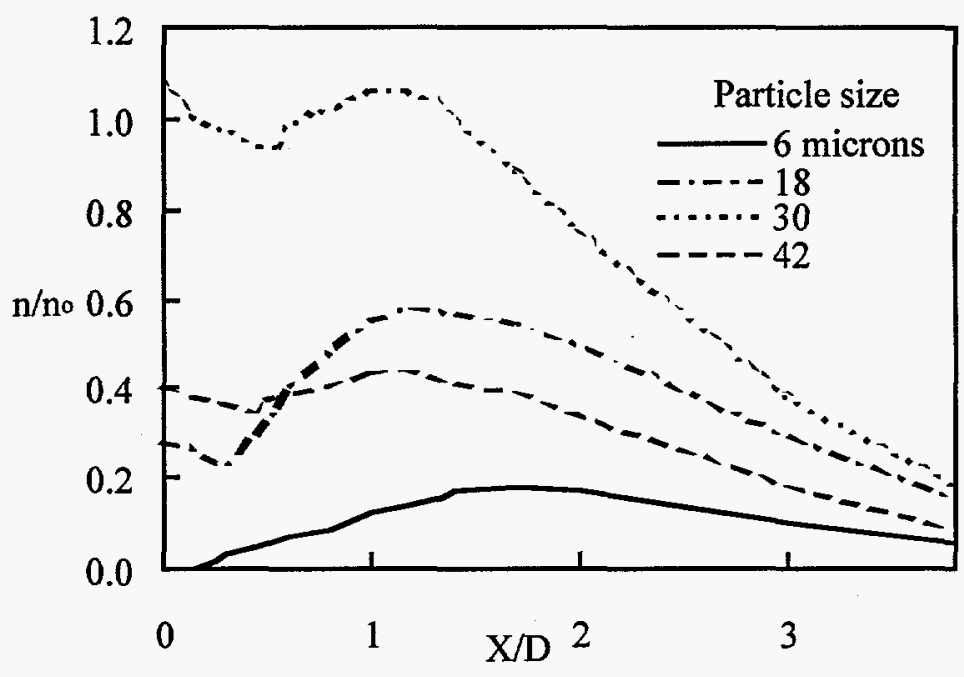

Figure 51 Centerline Particle Number Densities in the Combustor for Each Size Group

\subsubsection{Effects of Seed Particle Inlet Location on Seed Vapor Dispersion}

To investigate the effect of the seed vapor injection location on the degree of seed vapor mixedness, a series of combustion flows were computed with seed vapor introduced at a single computational cell at the inlet plane. Sixteen cases were computed for various single-cell seed vapor injection locations. For all these cases, flow and combustion patterns in the combustor remained relatively the same. Inlet coal-gas flow impinges with oxidizer jets, which are injected in a counter-flow direction. The impingement causes vortices to form right after the oxidizer jet slots, and flow accelerates through a necking area between the vortices. Combustion takes place where coal-gas and oxidizer mix, and the mixing takes place mainly around the vortices. Gas temperature increases along the main flow direction as combustion continues to take place and reaches a final flame temperature near the combustor exit. However, gas is cool inside the vortices, where it is mainly oxidizer. Figure 52 shows computed dispersion patterns of seed vapor in the combustor for a case in which the seed vapor injection location is next to the wall, $y_{j} / D=0.03$. The contour numbers in the figure represent normalized seed vapor concentration, $\mathrm{Y}_{\mathrm{sv}} / \mathrm{Y}_{0}$, and the reference concentration $\mathrm{Y}_{\mathrm{o}}$ is an arbitrarily selected value, 0.001 . Seed vapor concentration at the single injection cell is 0.02 . It appears that the dispersion of seed vapor is greatly affected by the gas flow patterns and the vortices enhance the dispersion. The seed vapor flows around one of the vortices, through the necking area, then, diffuses across the chamber. Some seed vapor trapped in the cool vortices near the wall is likely to condense on the wall because of the low temperature. 


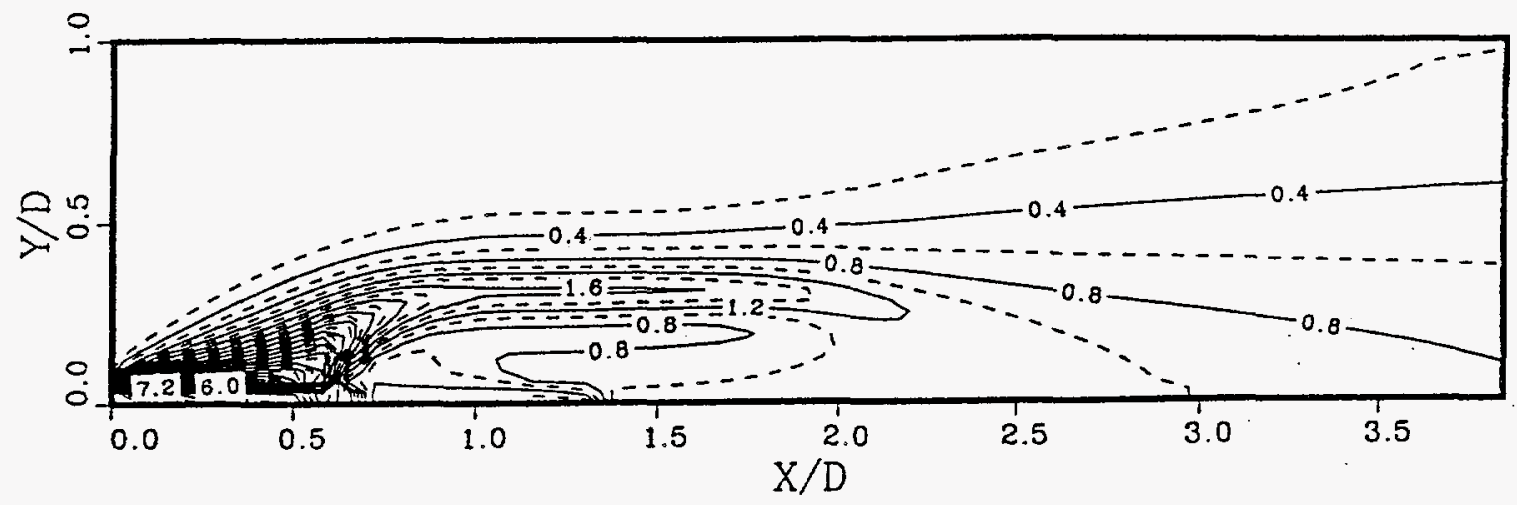

Figure 52 Seed Vapor Distribution in the Combustor for a Single-Cell Particle Injection at $\mathrm{y}_{\mathbf{j}} / \mathrm{D}=\mathbf{0 . 0 3}$

Figure 53a shows cross-sectional profiles of seed vapor concentration at four axial locations, $x / D=0,0.66,1.00$, and 3.81, for the case, $y_{j} / D=0.03$. Figures $53 \mathrm{~b}$ and $53 \mathrm{c}$ show similar seed vapor profiles for two other single-cell, seed-vapor-injection locations, $y_{j} / D=0.23$ and 0.5 , respectively. In general, seed vapor diffuses from injection location to the walls as the gas flows downstream. At the oxidizer jet slot location, $x / D=0.66$, seed vapor is apparently pushed toward the combustor center by the jets, and a significant amount of seed vapor is present near the oxidizer jet slots where gas temperature is low and axial velocity is negative. In this region, seed vapor may be trapped and condense on the walls. At the exit, $x / D=3.81$, seed vapor appears to have been spread across the channel. For the symmetric injection case (Fig. 53c), the dispersion of the seed vapor is very effective, the mixing is nearly complete, and the exit seed vapor concentration is relatively uniform. For the asymmetric injection cases (Figs. 53a and 53b), the mixing is not yet complete, but a considerable amount of seed vapor has penetrated into the far side (away from the injection side) of the combustor.

Figure 54 shows some interesting seed vapor dispersion patterns in the combustor. To show the degree of seed vapor mixing in the combustor, a seed mixedness measure is defined as the standard deviation of seed vapor concentration divided by its mean in a cross-sectional area. The smaller the mixedness measure, the higher or more complete the mixing. Exit seed mixedness increases (mixedness measure decreases) as the seed injection location moves toward the midpoint of the combustor, $y_{j} / D=0.5$, as shown in Fig. 54. The trapped seed vapor near the oxidizer jet slots, $x / D=0.66$, increases as the seed injection moves toward the combustor walls. In other words, seed-vapor injection near the combustor wall is more likely to condense on the walls, and seed vapor injected near the combustor center may have better mixing and less condensation. 


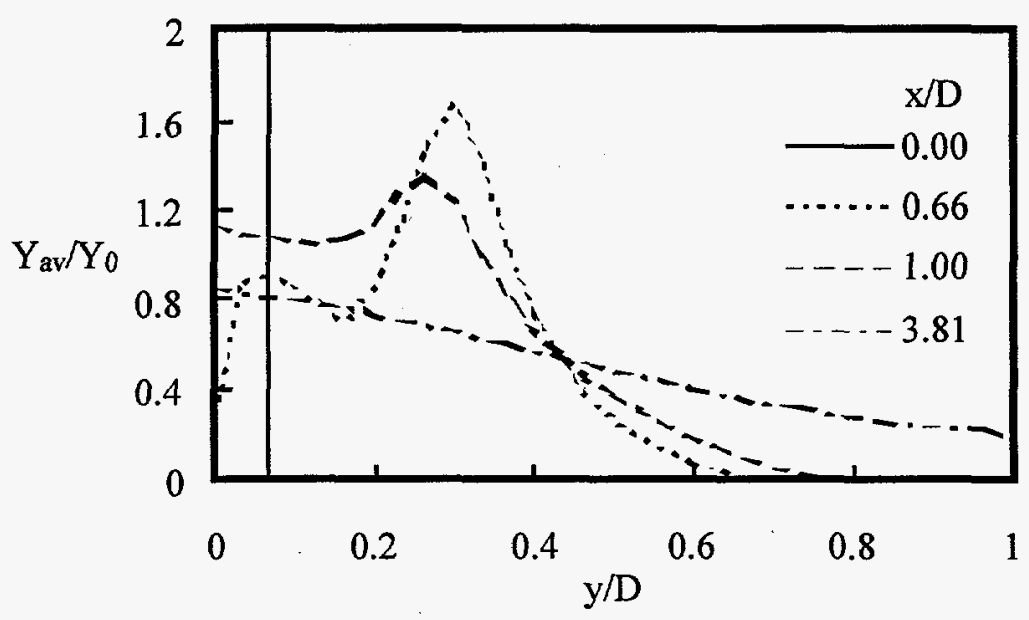

(a) $y_{j} / D=0.03$

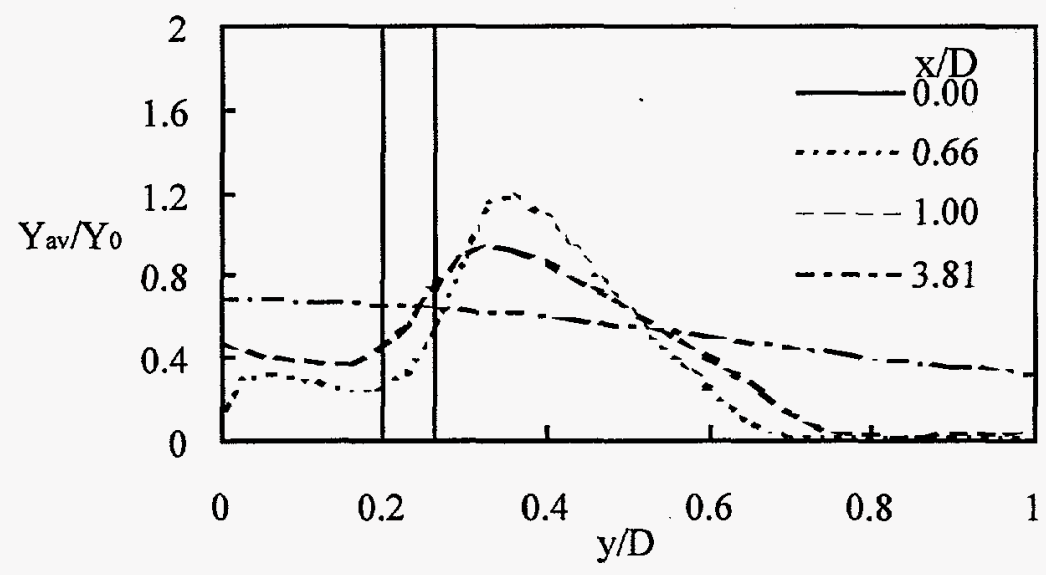

(b) $y_{j} / D=0.23$

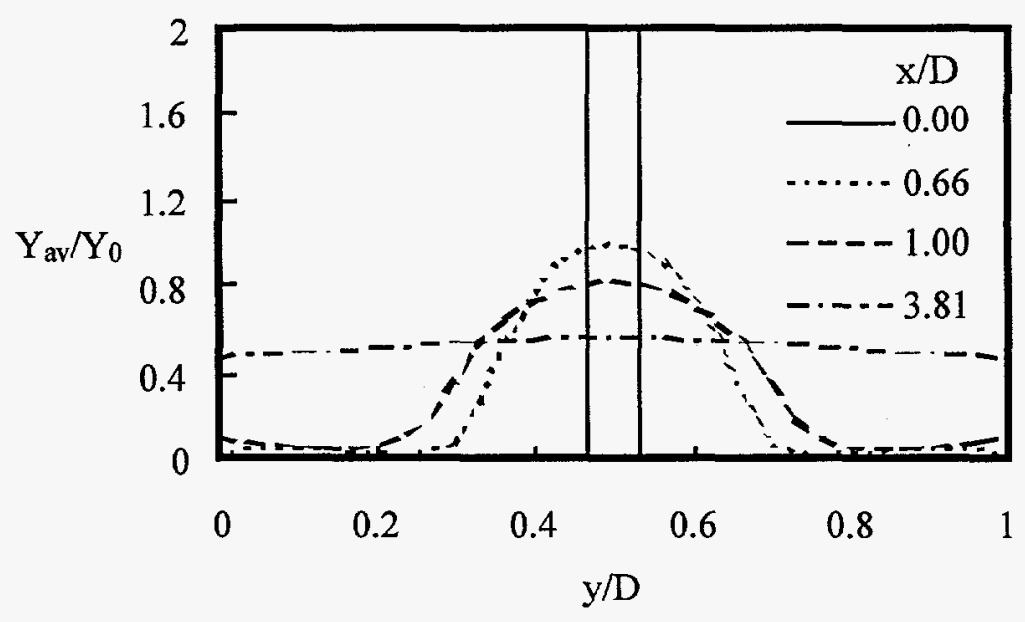

(c) $y_{j} / D=0.5$

Figure 53 Seed-Vapor Profiles in the Combustor for Various Single-Cell Injection Locations 


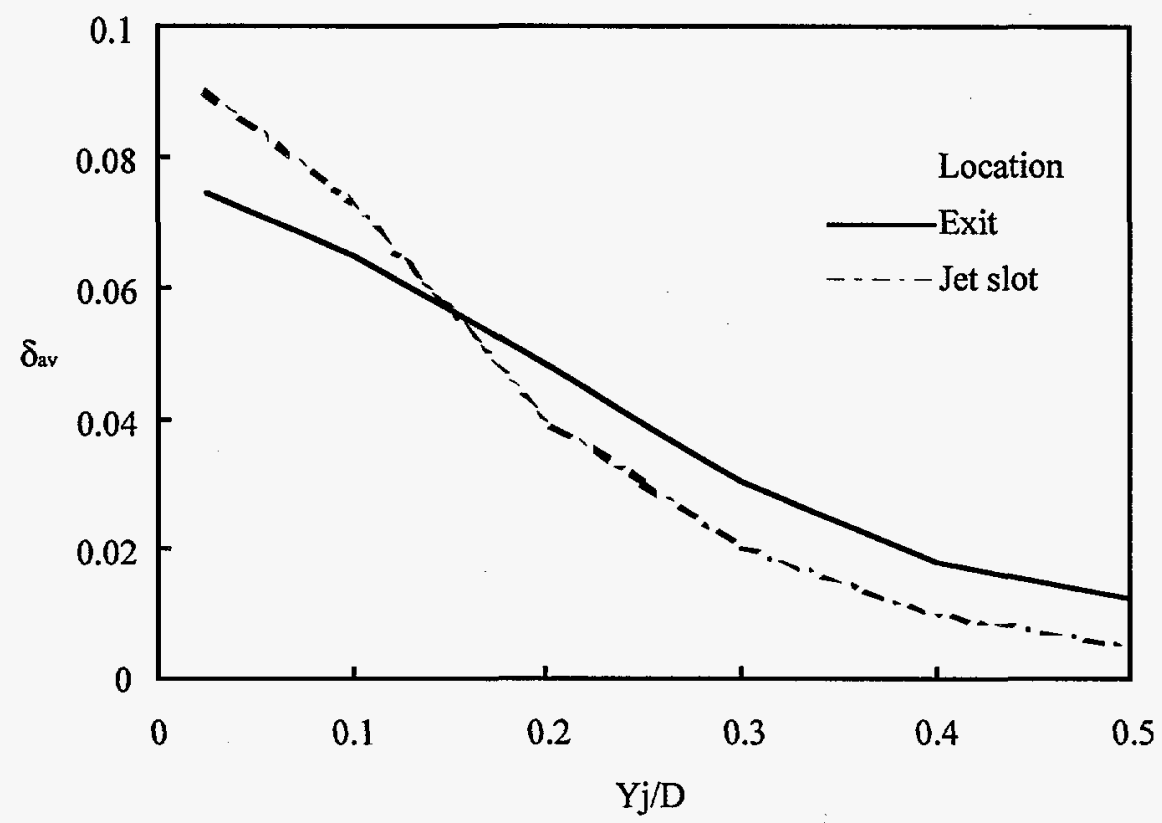

Figure 54 Seed-Vapor Mixedness at the Combustor Exit for Various Single-Cell Injection Locations

\subsection{Seed Ionization and Development of Electrical Conductivity Patterns in the Plasma}

The combustion code with the gas ionization submodel was used to study the processes of seed particle vaporization and gas ionization in the combustor. The study investigated the change of seed particle size, the dispersion of seed vapor, and the ionization of seed vapor under common reacting flow conditions, as was shown in Table 11. Computations were performed for a uniform distribution of seed particles across the combustor inlet. The mean inlet particle size was $34 \mu \mathrm{m}$. The particle loading was $1 \%$ of seed material in mass.

A combustion computation including seed particle dynamics and vaporization over the combustion chamber was performed. Point-by-point results of the computation were used by the gas ionization submodel to determine electron and ion number densities over the interior of the combustor as well as the development of gas conductivity within the combustor. Results of a sample baseline computation for electron number density and gas conductivity are shown in Fig. 55. Contours of the log base 10 of electron number density are shown in Fig. 55a. Near the chamber entry at $\mathrm{x} / \mathrm{D}=0$ with the oxidizer jets at $\mathrm{x} / \mathrm{D}=0.66$, electron number density is low and conductivity is negligible. As seed particles vaporize and the gas temperature becomes elevated due to combustion, the extent of ionization increases downstream to the point where an electron number density on the order of $10^{21}$ electrons $/ \mathrm{m}^{3}$ and conductivities on the order on $10 \mathrm{mho} / \mathrm{m}$ are seen (Fig. 55b). 


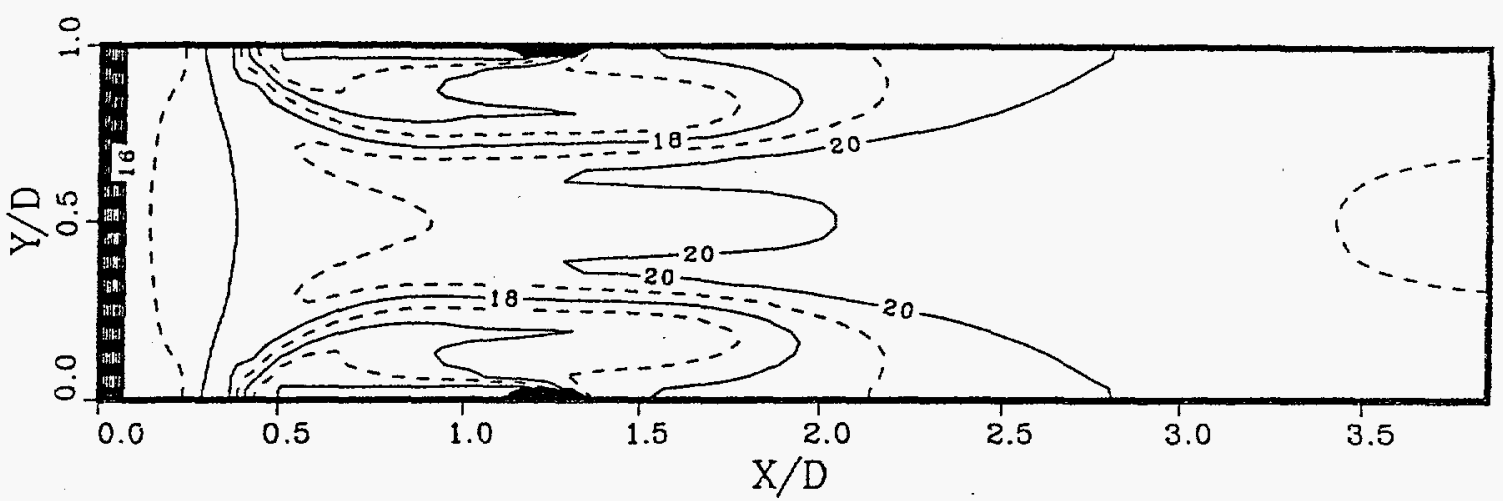

(a) electron number density

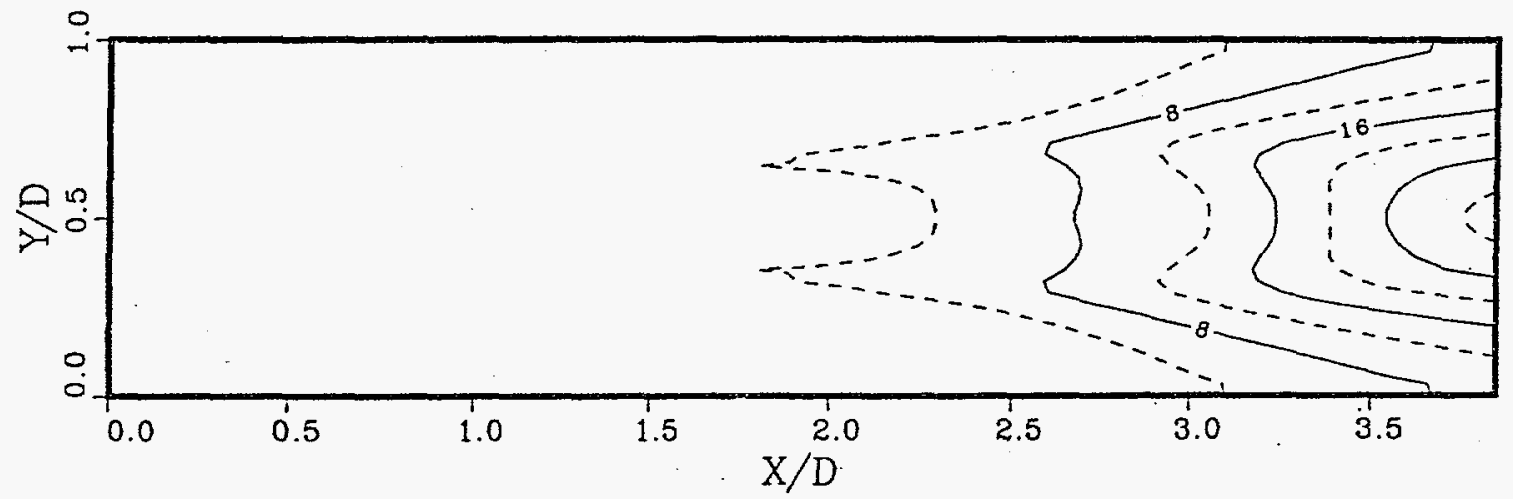

(b) electric conductivity

Figure 55 Gas Ionization Patterns in the Combustor 


\section{MULTIPHASE FLOW IN THE CONVERGING NOZZLE}

Two types of studies were done for the converging nozzle. The first study focused on nozzle geometry and the continuation of the two-phase reacting and ionizing flow from the second-stage combustor as it accelerated through the nozzle. Uniformity of flow property development, seed and slag particle impact on the nozzle walls, and heat loss from the nozzle were studied for three nozzle geometries. The second study focused on iron oxide injection in the nozzle. Iron oxide was injected into the converging nozzle section of the CDIF MHD power train testing facility. The injection was intended to cover the cathode wall (the bottom wall) of the MHD channel uniformly with iron oxide to protect the cathode wall from erosion. When this process was not successful at CDIF, ANL undertook a CFD study of the process to better understand the mechanisms in the nozzle flow that could lead to problems in achieving the desired result. ANL adapted the ICOMFLO computer code to simulate the flow of iron-oxide particles in the converging nozzle leading to the MHD channel [30]. The specific objective of the simulation was to investigate the effects of particle size and injection velocity on the spreading (or distribution) of iron oxide in a plane parallel to and slightly above the bottom nozzle wall, which is the cathode wall in the MHD channel. An additional objective was to investigate the effect of particle size on heating delay to determine if residence time in the nozzle was sufficient to melt the iron-oxide particles over the size range of interest.

\subsection{The Converging Nozzle}

Figure 56 shows the simulated volume in the three planes of the nozzle geometry: the y-z plane, which is perpendicular to the primary flow direction, with y running from one side wall to the other, and $\mathrm{z}$ running from the nozzle bottom to the top wall; the $\mathrm{z}-\mathrm{x}$ plane, in which $\mathrm{x}$ runs along the nozzle length in the primary flow direction; and the $x-y$ plane, which constitutes the computational domain of the two-dimensional simulation. The spreading of the iron-oxide jets in the z-direction is assumed to be relatively slow compared to spreading, dispersion, and turning in the $\mathrm{x}$-direction, because the main gas flow from the nozzle inlet is nearly parallel in the $\mathrm{x}$-direction and of relatively high velocity (approximately in the range $100-900 \mathrm{~m} / \mathrm{s}$ over the nozzle length). Nearly complete vaporization of the slurry oil is assumed to occur near the injectors, leaving a spray of iron oxide particles to be turned and dispersed into the downstream by the main nozzle gas flow. To account for spreading in the z-direction, the simulated volume is assumed to be approximately twice the iron-oxide jet diameter. The two-dimensional computational domain is an $x-y$ plane slice taken to be approximately halfway up through the $\mathrm{z}$-direction of the computational volume.

Although the problem has some three-dimensional aspects, the cost, development time, and data processing time for a full three-dimensional simulation made such an approach to the problem not feasible. The ICOMFLO computer code was already set up to simulate the flow of a mixture of gas and condensed phase (either particles or droplets) in the current TRW converging nozzle configuration. By making some simplifying assumptions and modifying the subroutine code to provide iron-oxide spray jet injection from the side walls of the nozzle, a two- 
dimensional computational approach to the problem was formulated that provides a physically reasonable characterization of the parametric trends and sensitivity of the iron-oxide jet injection. However, due to the simplifying assumptions, the results are intended to predict mainly qualitative trends of the flow patterns.

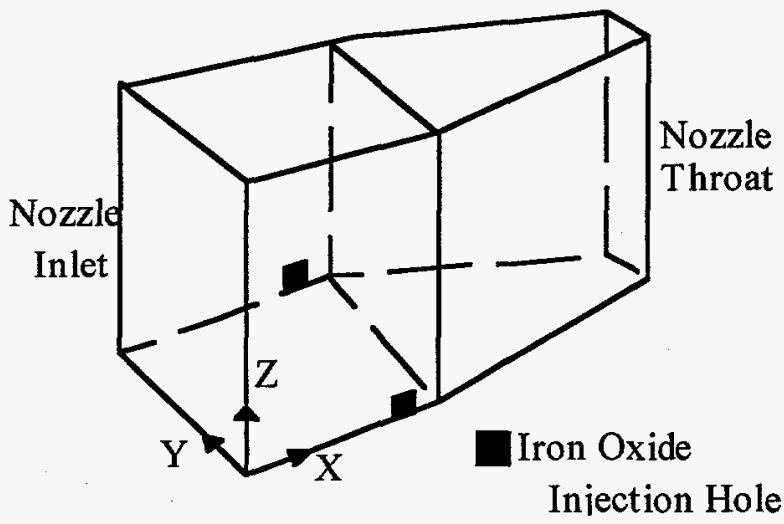

(a) Geometry of Nozzle \#1

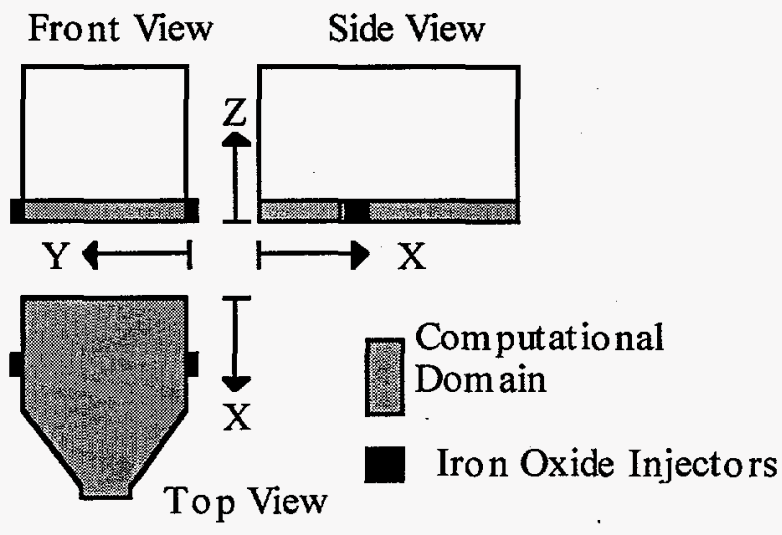

(b) 2-D Computational Domain

Figure 56 Geometry of Nozzle \#1 and its Computational Domain

\subsection{Flow Patterns in the Converging Nozzle (No Iron Injection)}

Nozzle flow calculations were made for three nozzle geometries suggested by TRW. The nozzle geometries have a rectangular cross-sectional area with a fixed width and variable height along the axial direction. The contours of the nozzle height are shown in Fig. 57. The first one is the present contour (or contour \#1), which has a 27-degree slope near the nozzle throat; the second one is alternative contour \#4, which has a 27-degree slope near the nozzle inlet, followed by a gradually decreasing nozzle height with 10-, 5-, and 3-degree slopes; and the third one is alternative contour \#5, which has a 14-degree slope at the nozzle inlet. For all three nozzle geometries, the length is $0.41 \mathrm{~m}$, the width is $0.29 \mathrm{~m}$, the inlet height is $0.21 \mathrm{~m}$, and the exit height is $0.0681 \mathrm{~m}$.

Table 12 shows the common nozzle inlet flow conditions that were used in the parametric calculations and general nozzle dimensions. The inlet gas pressure is $5.7 \mathrm{~atm}$; the inlet gas temperature is $2800 \mathrm{~K}$; the average particle size is $60 \mu \mathrm{m}$; the mass fraction of particles in the flow is $1 \%$; and Mach number at the nozzle throat is 1 . In these calculations, the primary convergence criteria are set by using the mass residuals of both the gas and particle phases. The cases presented below were considered converged when the average of mass residuals for both phases was below $10^{-9}$. The grid system for the \#1 nozzle uses 46 by 32 cells, and the grid systems for the $\# 4$ and $\# 5$ nozzles use 53 by 42 cells, including blocked cells. Finer grid spacing is defined in the throat section, where the flow is choked and flow properties change rapidly with 
the area change. Calculations were made to compare particle-wall deposition rates, gas residence times, and convective heat losses for the three nozzle geometries.

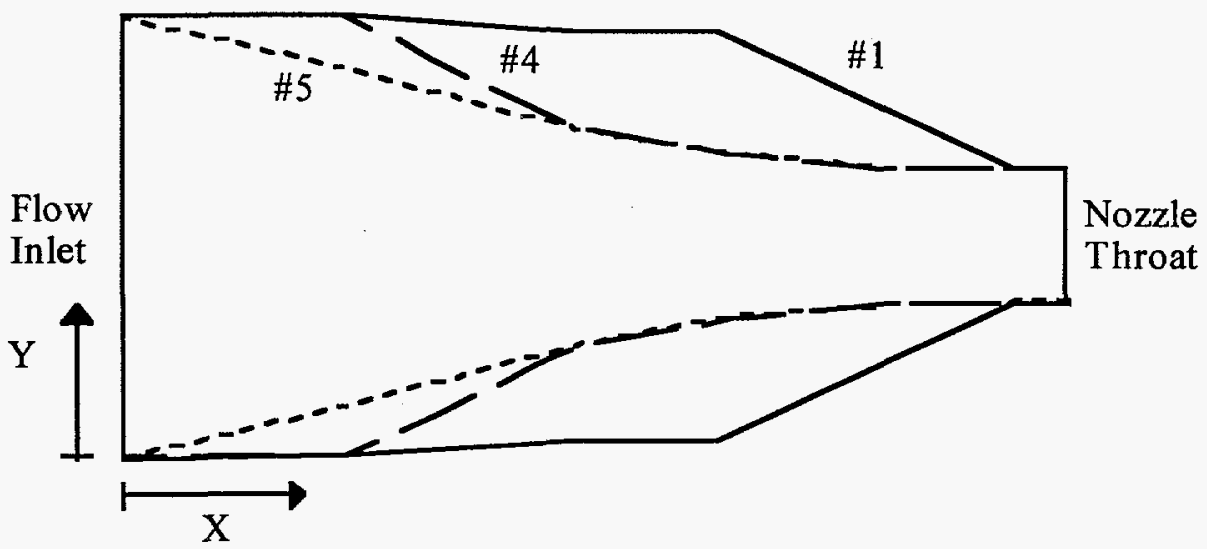

Figure 57 Geometric Contours of Nozzles \#1, \#4, and \#5

Table 12 Nozzle Flow Conditions and Dimensions

\begin{tabular}{|l|r|}
\hline Inlet Pressure $(\mathrm{atm})$ & 5.7 \\
\hline Inlet Gas Temperature $(\mathrm{K})$ & 2800 \\
\hline Mach Number at the Throat & 1 \\
\hline Particle Diameter $(\mu \mathrm{m})$ & 60 \\
\hline Inlet Particle Velocity $(\mathrm{m} / \mathrm{s})$ & 160 \\
\hline Bulk Particle Mass Fraction $(\%)$ & 1 \\
\hline Specific Heat Ratio & 1.12 \\
\hline Throat Area/Inlet Area & 0.33 \\
\hline Nozzle Width $(\mathrm{m})$ & 0.29 \\
\hline
\end{tabular}

\subsubsection{Effect of Nozzle Geometries on Flow Development}

Nozzle geometry has a decisive effect on the development of gas pressure, gas temperature, and Mach number in the nozzle. Calculations were made to predict gas/particle flow patterns and particle-wall deposition rates for various nozzle contours. Gas pressure, temperature drop, Mach number, particle-number density, gas velocity, and particle velocity in the three nozzle geometries were computed for comparison.

Figure 58 compares the development of pressure in the nozzles. In all three nozzle geometries, gas pressure decreases from $5.7 \mathrm{~atm}$ at the inlet to about $3.5 \mathrm{~atm}$ at the throat. The results indicate that the overall flow development for nozzle contours \#4 and \#5 is much more 
uniform over the length of the nozzle than that for nozzle contour \#1. Similar trends were found for the development of gas temperature and Mach number. Calculations predicted that gas temperature will drop about $120 \mathrm{~K}$ from the inlet temperature by the time the flow reaches the nozzle throat, and flow Mach number will increase from 0.17 at the inlet to 1 at the throat. Among the three nozzles, the \#5 nozzle has the smoothest flow development in the nozzle.

Figure 59 compares gas flow patterns in the three nozzle geometries. In all three nozzles, the gas velocity increases from about $180 \mathrm{~m} / \mathrm{s}$ at the inlet to about $900 \mathrm{~m} / \mathrm{s}$ at the throat. Flow patterns in the \#1 nozzle show significant differences compared to those in the \#4 and \#5 nozzles, especially near the nozzle throat. In the \#1 nozzle, gas tends to flow toward the centerline at the throat due to the sharp flow angle and high pressure gradient, which effectively reduces the nozzle throat area. Inlet gas velocity, which is determined by satisfying the choking condition $(M=1)$ at the throat, changes slightly for various nozzle contours because of the change of the effective throat area. The computed inlet gas velocity was $175 \mathrm{~m} / \mathrm{s}$ for the $\# 1$ nozzle and $182 \mathrm{~m} / \mathrm{s}$ for the \#4 and \#5 nozzles.

The simulation showed that particle flow patterns were different from gas flow patterns. Particles generally maintain a parallel flow direction throughout the nozzle length, and a large fraction of particles near a wall collide with and deposit on the wall. Figure 60 compares the accumulated particle deposition on the nozzle walls for the three configurations. Among the three nozzles, the \#5 nozzle has the lowest particle wall deposition rate, especially near the nozzle throat. 


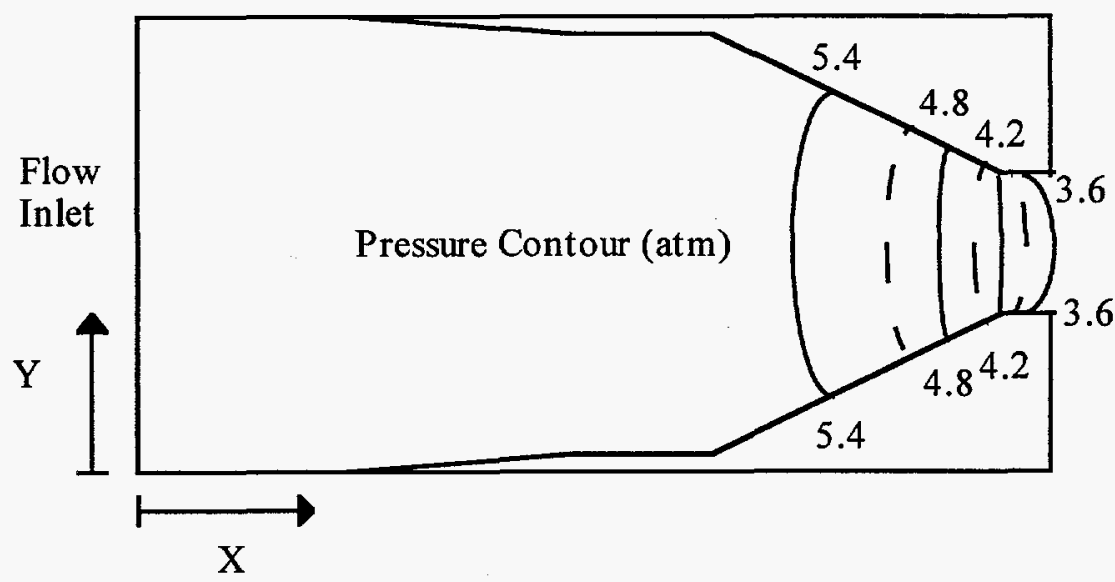

(a) Nozzle \#1

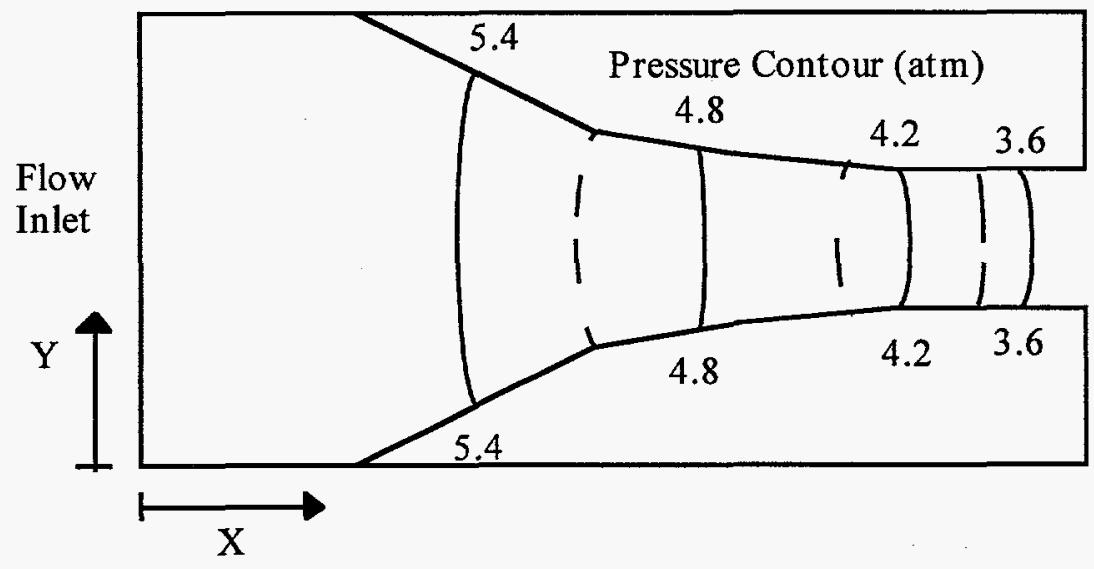

(b) Nozzle \#4

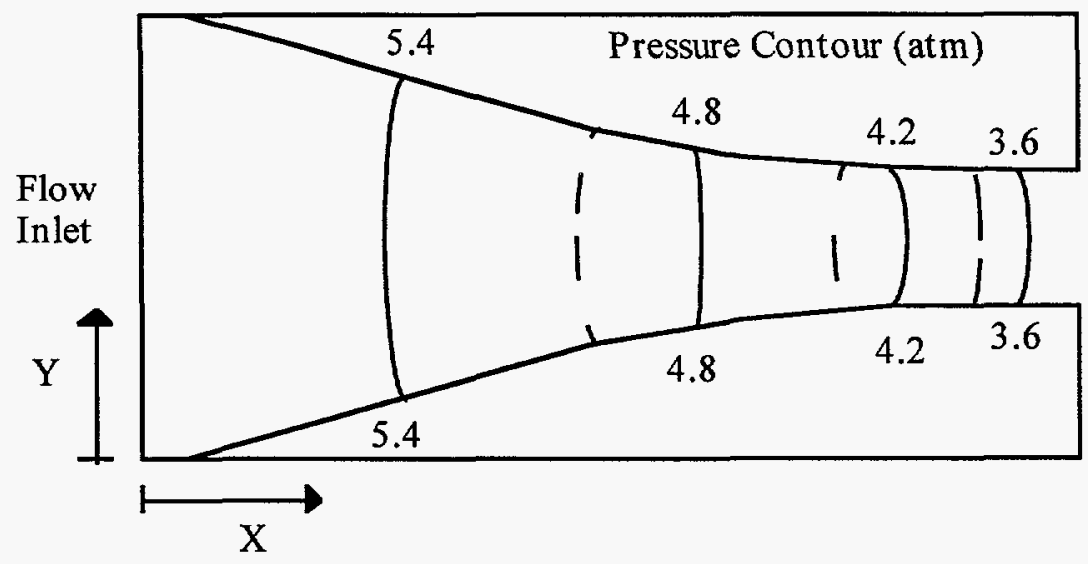

(c) Nozzle \#5

Figure 58 Development of Gas Pressure in Various Nozzles 


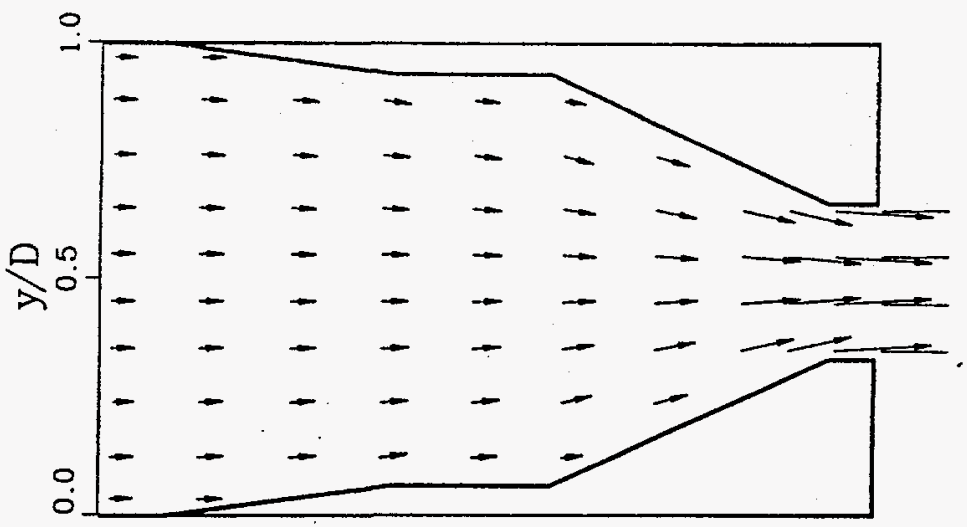

(a) nozzle \#1

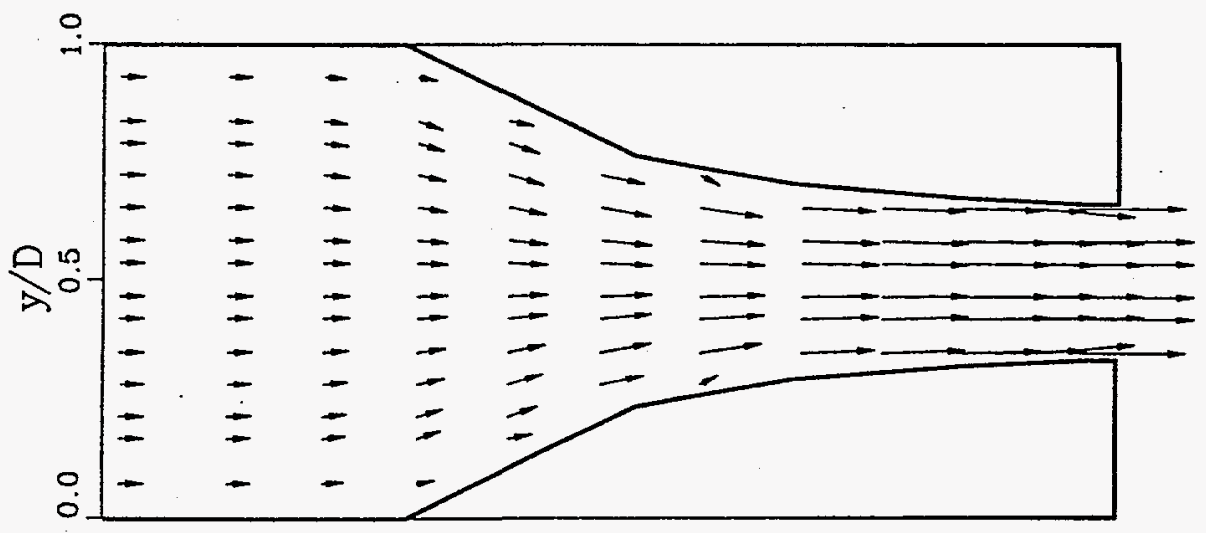

(b) nozzle \#4

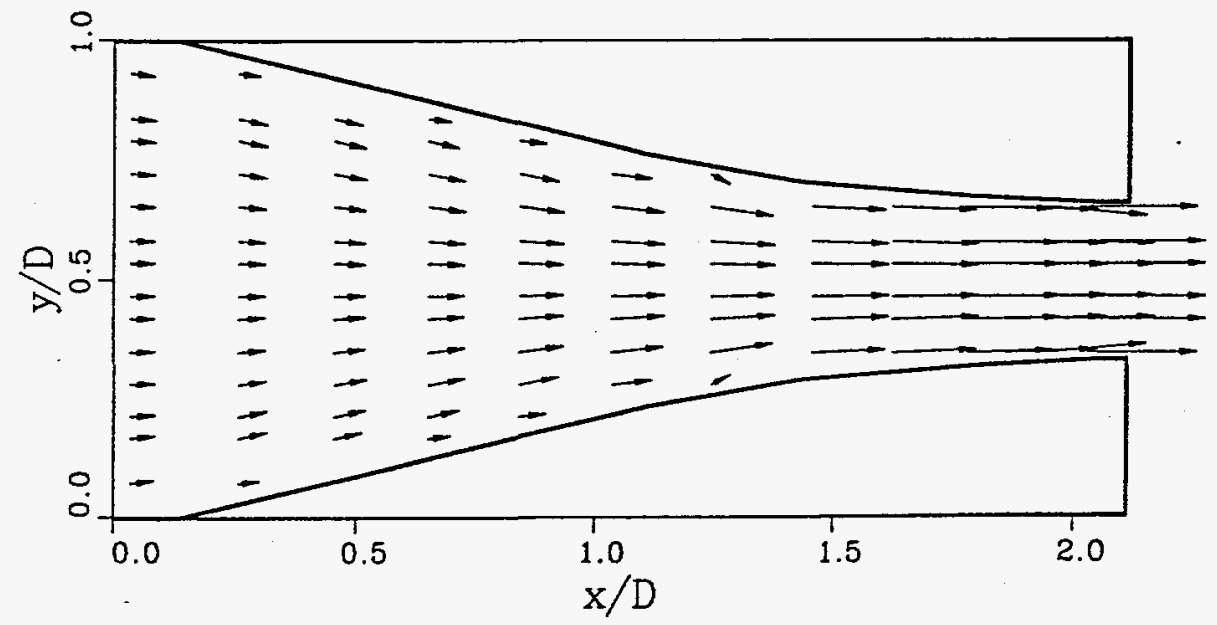

(c) nozzle \#5

Figure 59 Gas Flow Patterns in Various Nozzles 


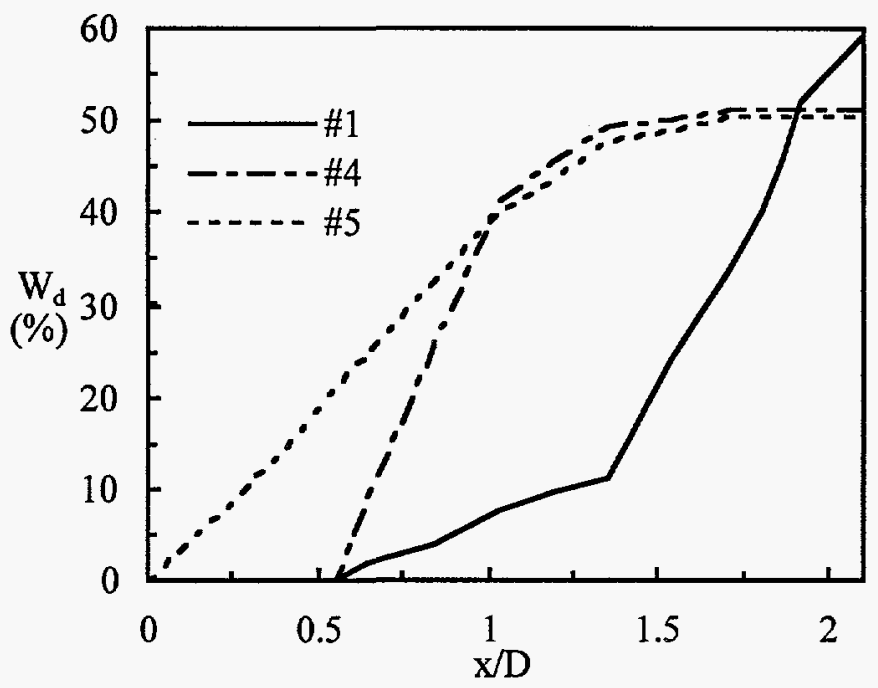

Figure 60 Particle Deposition in Various Nozzle Geometries

\subsubsection{Residence Time and Heat Loss}

Change of nozzle geometry also has some impact on residence time and heat loss in the nozzle. Figure 61 compares gas and particle residence times for the three nozzle contours. Plotted is the accumulated residence time for a representative gas species or particle as it travels through the nozzle at the centerline. These results were obtained from the detailed twodimensional flow simulation.

The effects of changing nozzle contour on wall heat transfer were estimated by using classical heat transfer theory combined with Nusselt correlations. For turbulent flow in tubes, the following equations are commonly used to calculate the surface heat transfer rate, $q$.

$$
\begin{aligned}
& q=h A\left(T-T_{w}\right) \\
& N u=h D / k \\
& N u=d \operatorname{Pr}^{0.6} \operatorname{Re}^{0.8} \\
& \operatorname{Pr}=\mu C p / k \\
& \operatorname{Re}=\rho U D / \mu
\end{aligned}
$$

In the above equations, $\mathrm{Nu}, \mathrm{Pr}$, and $\mathrm{Re}$ are Nusselt, Prandtl, and Reynolds numbers, respectively. The properties of air at a temperature of $2600 \mathrm{~K}$ are listed as follows: 


$$
\begin{aligned}
& \operatorname{Pr}=1 \\
& \mu=7.5 \times 10^{-5} \mathrm{~kg} / \mathrm{m}-\mathrm{s} \\
& \mathrm{k}=0.14 \mathrm{~W} / \mathrm{m}-\mathrm{K} \\
& \mathrm{Cp}=1700 \mathrm{~J} / \mathrm{kg}-\mathrm{K}
\end{aligned}
$$

Three nozzle geometries suggested by TRW each have an inlet area of $0.00597 \mathrm{~m}^{2}$ and a throat area of $0.00198 \mathrm{~m}^{2}$. Total surface areas of the $\# 1, \# 4$, and $\# 5$ nozzles are $0.3587,0.3289$, and $0.3191 \mathrm{~m}^{2}$, respectively. Average hydraulic diameters of the \#1, \#4, and \#5 nozzles are $0.2315,0.2122,0.206 \mathrm{~m}$, respectively. Calculations conducted in the previous section show that the inlet, average, and throat pressures are 5.7, 4.65, and $3.6 \mathrm{~atm}$, respectively; the inlet, average, and throat temperatures are 2800,2725 , and $2650 \mathrm{~K}$ respectively; the inlet gas density is $0.7 \mathrm{~kg} / \mathrm{m}^{3}$; and the average gas density is about $0.6 \mathrm{~kg} / \mathrm{m}^{3}$. Gas residence times for the \#1, \#4, and $\# 5$ nozzles are $1.95,1.33$, and $1.22 \mathrm{~ms}$, respectively. Average gas velocities in the \#1, \#4, and $\# 5$ nozzles are 223,328 , and $355 \mathrm{~m} / \mathrm{s}$, respectively. The inlet gas velocities for $\# 1$, \#4, and $\# 5$ nozzles are $175.5,182$, and $182 \mathrm{~m} / \mathrm{s}$. Mass flow rates in the \#1, \#4, and \#5 nozzles are 7.33, 7.61 , and $7.61 \mathrm{~kg} / \mathrm{s}$. Figure 61 compares gas and particle residence times along the nozzle length for three nozzle contours. Gas and particle residence times in the \#1 nozzle are longer than those in the \#4 and \#5 nozzles.

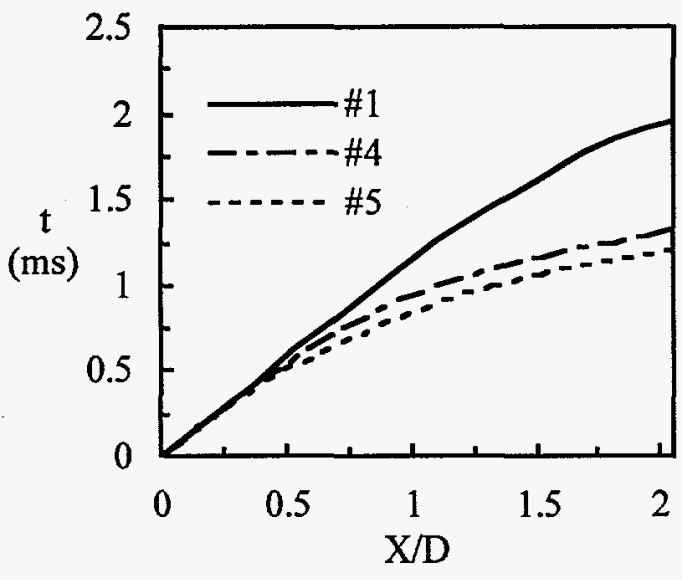

(a) Gas

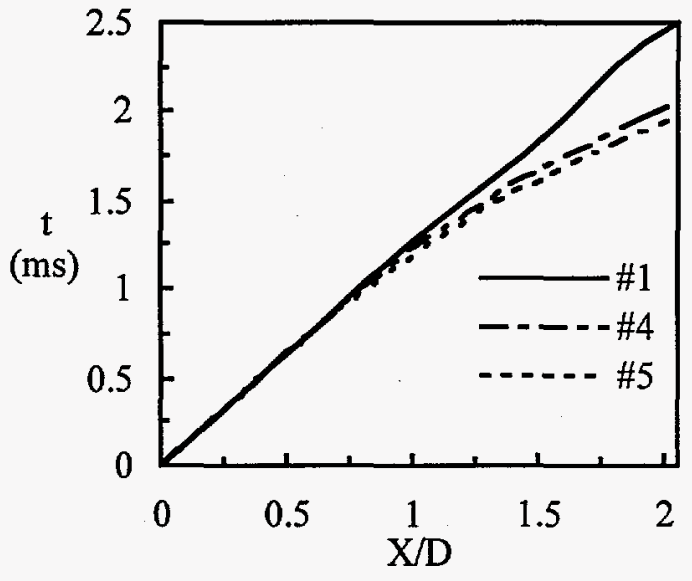

(b) Particle

Figure 61 Gas and Particle Residence Times in the Nozzles

CDIF measured a $0.43 \mathrm{MW}$ heat loss in the present nozzle \#1. From Eq. 6.3, knowing the heat loss $\mathrm{q}$ and assuming an average temperature difference of $1700 \mathrm{~K}$ between the gas and nozzle walls, the convection conductance $h$ is calculated as $700 \mathrm{~W} / \mathrm{m}^{2}-K$. Substituting $h, D$, and $\mathrm{k}$ into Eq. 6.4, the Nusselt number in the \#1 nozzle is computed as 1157 . From Eq. 6.5, the Reynolds number is calculated as $4.13 \times 10^{5}$. Now, substituting $\mathrm{Nu}, \mathrm{Pr}$, and $\mathrm{Re}$ into Eq. 6.3, an empirical constant $d=0.037$ is obtained. For the \#4 nozzle, the Reynolds number is $5.57 \times 10^{5}$, the Nusselt number is 1462 , the convection conductance is $964 \mathrm{~W} / \mathrm{m}^{2}-\mathrm{K}$, and the estimated 
heat loss is $0.54 \mathrm{MW}$. For the \#5 nozzle, the Reynolds number is $5.85 \times 10^{5}$, the Nusselt number is 1520 , the convection conductance is $1033 \mathrm{w} / \mathrm{m}^{2}-\mathrm{K}$, and the estimated heat loss is $0.56 \mathrm{MW}$. The $0.43 \mathrm{MW}$ heat loss in the \#1 nozzle lowers the exit gas temperature by $33 \mathrm{~K}$. The $0.56 \mathrm{MW}$ heat loss in the $\# 5$ nozzle lowers the exit gas temperature by $43 \mathrm{~K}$. Table 13 summarizes the calculation results for the three nozzle contours.

Table 13 Comparison of Flow Properties for Various Nozzle Geometries

\begin{tabular}{|l|r|r|r|}
\hline Nozzle Contour & \multicolumn{1}{|c|}{$\# 1$} & \multicolumn{1}{|c|}{$\# 4$} & \multicolumn{1}{|c|}{$\# 5$} \\
\hline Surface Area $\left(\mathrm{m}^{2}\right)$ & 0.3587 & 0.3289 & 0.3191 \\
\hline Diameter $(\mathrm{m})$ & 0.2315 & 0.2122 & 0.2060 \\
\hline Gas Residence Time $(\mathrm{ms})$ & 1.95 & 1.33 & 1.22 \\
\hline Average Velocity $(\mathrm{m} / \mathrm{s})$ & 223 & 328 & 355 \\
\hline Inlet Velocity $(\mathrm{m} / \mathrm{s})$ & 175.5 & 182 & 182 \\
\hline Mass Flow Rate $(\mathrm{kg} / \mathrm{s})$ & 7.33 & 7.61 & 7.61 \\
\hline Heat Loss $(\mathrm{MW})$ & 0.43 & 0.54 & 0.56 \\
\hline Reynolds Number $\left(\mathrm{x} 10^{5}\right)$ & 4.13 & 5.57 & 5.85 \\
\hline Nusselt Number & 1157 & 1462 & 1520 \\
\hline
\end{tabular}

\subsection{Iron Oxide Injection in the Converging Nozzle}

The information about the iron-oxide injection, including the injector configuration, injection location, and injection flow conditions, was provided by TRW. The injection location is shown in Fig. 56, and some major nozzle flow and iron-oxide jet flow properties are listed in Table 14. The iron-oxide mixture is injected at room temperature at a mass flow rate of $0.0691 \mathrm{~kg} / \mathrm{s}$, in which the particle flow rate is $0.0154 \mathrm{~kg} / \mathrm{s}$. In the jet, the gas velocity is $61.5 \mathrm{~m} / \mathrm{s}$, and the velocity of the suspended particles is in the range $5-30 \mathrm{~m} / \mathrm{s}$. The particle size range is 15-60 $\mu \mathrm{m}$. On the basis of flow conditions, calculations were made to assess the effects of particle size and injection velocity on the dispersion of the iron oxide particles in the \#1 nozzle.

This effect of particle size on the particle velocity field is illustrated in Fig. 62. Velocity vectors for the gas flow are shown in Fig. 62a. Velocity vector fields for tests with two different particle diameters are shown in Figs. $62 \mathrm{~b}$ and $62 \mathrm{c}$ for particles with a $15-$ and a $60-\mu \mathrm{m}$ diameter, respectively. The iron oxide particles enter the system with a velocity primarily directed toward the centerline of the nozzle. The main flow of hot gas through the nozzle will turn the particles into the downstream through the action of drag forces, as shown in Figs. $62 \mathrm{~b}$ and $62 \mathrm{c}$. However, because the main gas flow itself is also turning toward the nozzle centerline in the converging nozzle, as shown in Fig. 62a, no component of the drag force acts against the component of particle velocity in the direction of the nozzle centerline; particles will, therefore, continue to move toward the nozzle center as they are accelerated into the downstream by the interaction of 
drag with the main gas flow. The turning rate and acceleration of iron-oxide particles into the downstream are functions of particle size and injection velocity, with larger particles turning and accelerating more slowly than smaller particles.

Table 14 Summary of Two-Phase Flow Properties in the \#1 Nozzle

\begin{tabular}{|l|r|}
\hline Nozzle Contour & $\# 1$ \\
\hline Inlet Gas Velocity $(\mathrm{m} / \mathrm{s})$ & 157 \\
\hline Inlet Gas Temperature $(\mathrm{K})$ & 2800 \\
\hline Total Jet Mass Flow Rate $(\mathrm{kg} / \mathrm{s})$ & 0.0691 \\
\hline Particle Jet Mass Flow Rate $(\mathrm{kg} / \mathrm{s})$ & 0.0154 \\
\hline Jet Gas Velocity $(\mathrm{m} / \mathrm{s})$ & 61.5 \\
\hline Jet Particle Velocity $(\mathrm{m} / \mathrm{s})$ & $5-30$ \\
\hline Particle Size $(\mu \mathrm{m})$ & $15-60$ \\
\hline Jet Particle Temperature $(\mathrm{K})$ & 300 \\
\hline Jet Gas Temperature $(\mathrm{K})$ & 300 \\
\hline Particle Melting Temperature $(\mathrm{K})$ & 1810 \\
\hline
\end{tabular}

Figures $63 \mathrm{a}$ and $63 \mathrm{~b}$ show particle number density distributions in the nozzle for two particle diameters, 15 and $60 \mu \mathrm{m}$, respectively. The numbers appearing on the number density contour lines are normalized by the inlet number density at the jets. Again, these results are the consequence of the effect of drag forces on smaller particles. Smaller particles are turned and accelerated more rapidly into the downstream than larger particles. Thus, very small particles may not have sufficient residence time to travel inward to the region inline with the nozzle throat. Therefore, a portion of them will collide with and collect on the converging nozzle walls in the nozzle configuration tested. Large particles tend to move collectively toward the center, as shown in Fig. 63b. Because the jets are located near the bottom wall of the nozzle effects of gravity and spreading of the jets may cause iron oxide particles to collide with and collect on the bottom nozzle wall in patterns similar to those shown in Fig. 63, depending on particle size. 


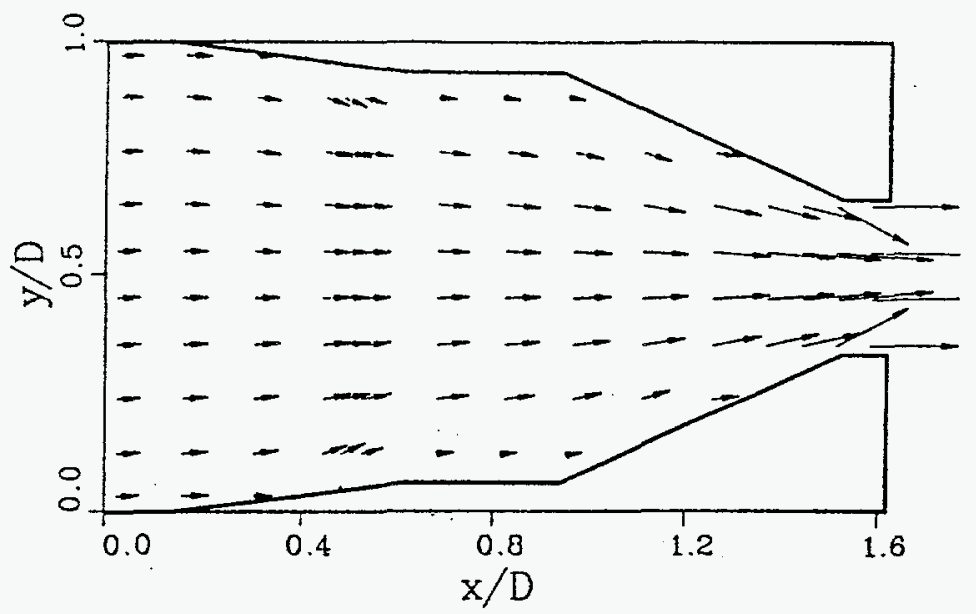

(a) gas velocity

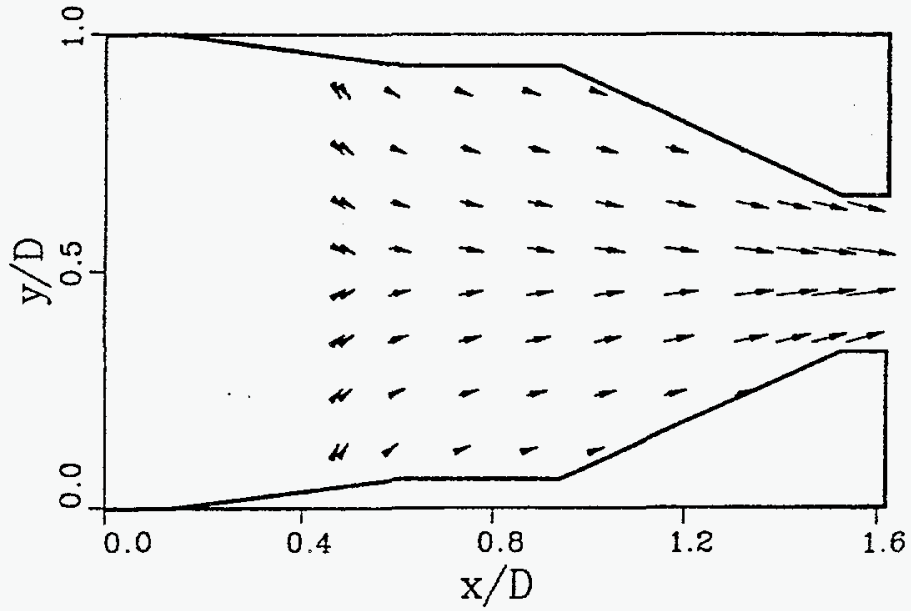

(b) particle velocity $(15 \mu \mathrm{m})$

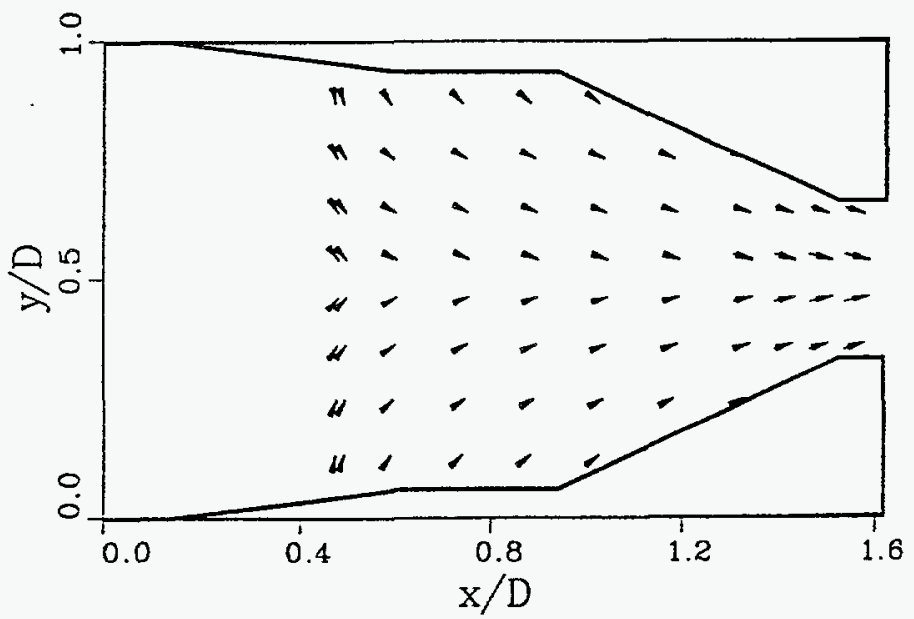

(c) particle velocity $(60 \mu \mathrm{m})$

Figure 62 Gas and Particle Flow Patterns in Nozzle \#1 


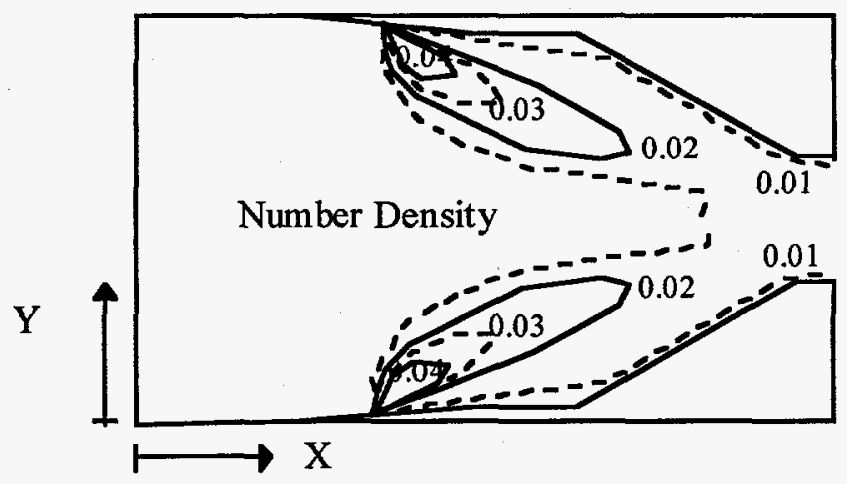

(a) $15-\mu \mathrm{m}$ particles

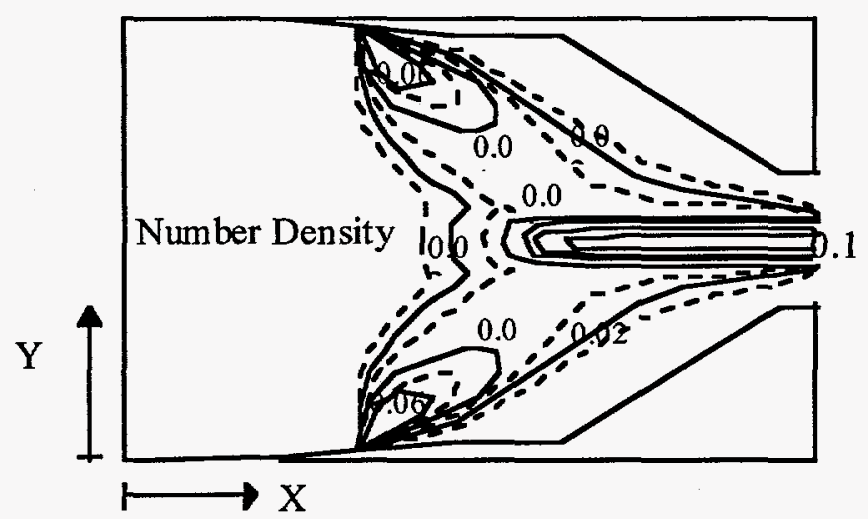

(b) $60-\mu \mathrm{m}$ particles

Figure 63 Dispersion of Particles in Nozzle \#1

Figures $64 \mathrm{a}$ and $64 \mathrm{~b}$ show particle-temperature distributions in the nozzle for two particle diameters, 15 and $60 \mu \mathrm{m}$, respectively. The numbers appearing on the temperature contour lines are normalized by the inlet gas temperature $(2800 \mathrm{~K})$. The iron oxide particles in the simulation are injected into the nozzle at room temperature and heated up by the surrounding hot gas through interfacial heat transfer, which depends largely on the temperature difference between two phases and surface area to volume ratio. Larger particles, having smaller surface-area-tovolume ratios, are heated more slowly than smaller particles. When particle temperature exceeds the melting temperature of iron oxide (normalized temperature 0.65 ), the particles transform from solid phase to liquid phase. Particles of $15-\mu \mathrm{m}$ diameter heat up rapidly in the nozzle and become liquid droplets in the nozzle as shown in Fig. 64a. On the other hand, particles of $60-\mu \mathrm{m}$ diameter remain solid particles in the nozzle as shown in Fig. 64b. The solid particles at the nozzle throat have a high velocity and therefore can cause erosion on the nozzle walls. 


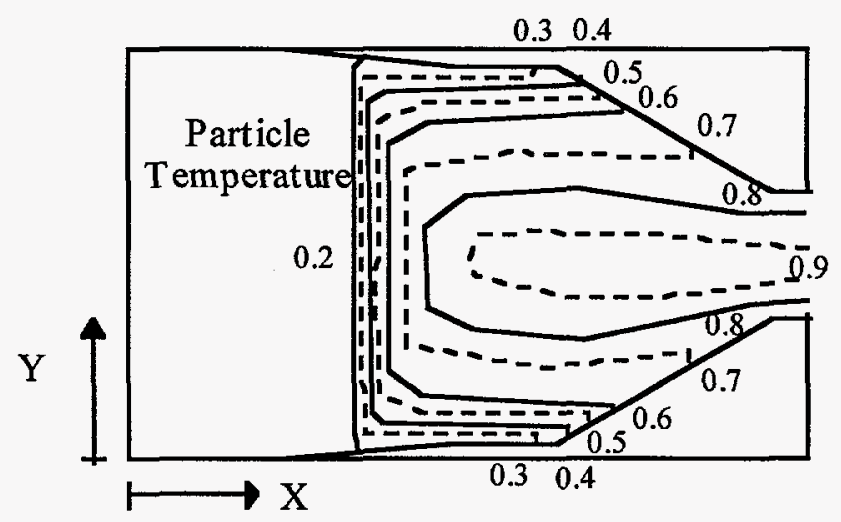

(a) $15-\mu \mathrm{m}$ droplets

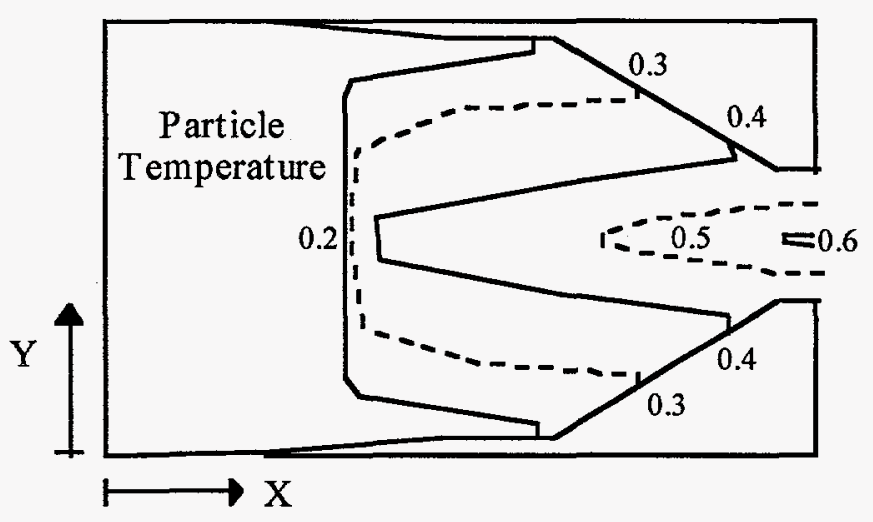

(b) $60-\mu \mathrm{m}$ droplets

\section{Figure 64 Distribution of Droplet Temperature in Nozzle \#1}

Figure 65 compares particle accumulation on the converging nozzle walls for three particle radii of 15,30 , and $60 \mu \mathrm{m}$, and two injection velocities of 30 and $5 \mathrm{~m} / \mathrm{s}$. At the $30-\mathrm{m} / \mathrm{s}$ injection velocity, most larger particles accumulate in the nozzle center (as shown in Fig. 63b) and few particles accumulate on the nozzle walls, but a significant amount of smaller particles (over $20 \%$ ) accumulate on the nozzle walls because they are more easily turned by the main stream toward the walls. Particles with a slower injection velocity may also accumulate on the nozzle walls to some extent (about 10\%).

Figure 66 compares the number density profiles of $60-\mu \mathrm{m}$ iron-oxide particles at three axial locations, $\mathrm{X} / \mathrm{D}=0.8,1.2$, and the nozzle throat. The number density in the figure is normalized by the iron oxide particle number density at the injection location. The comparison shows that iron oxide particles penetrate the crossflow by their own momentum in the $y$-direction at the injection jet, as they flow downstream in the nozzle. Most $60 \mu \mathrm{m}$ iron-oxide particles concentrate at the nozzle centerline as they flow through the nozzle throat. 


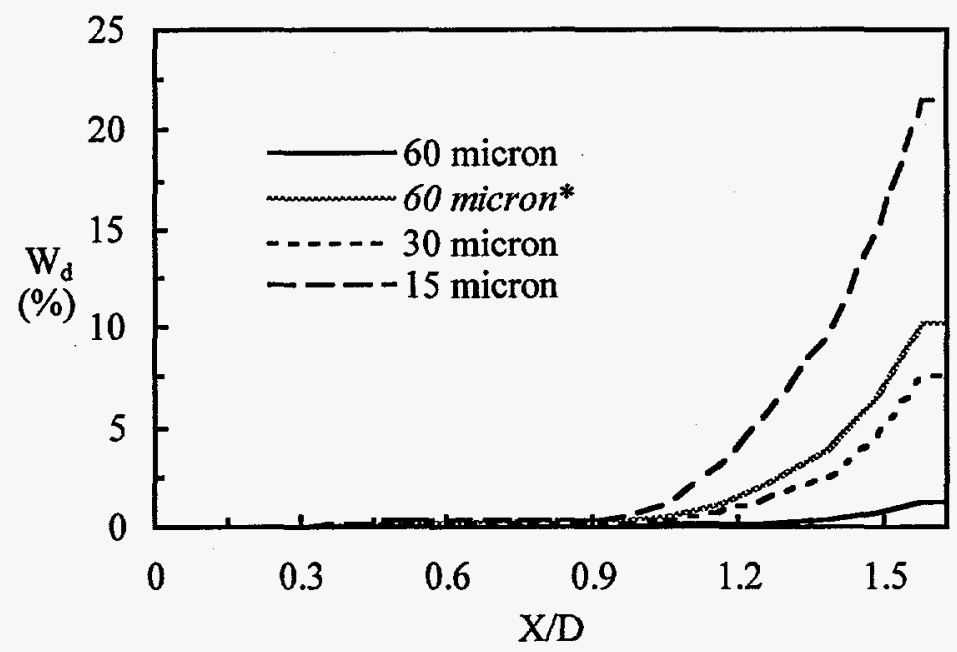

*Particle Injection Velocity $=5 \mathrm{~m} / \mathrm{s}$, all others $30 \mathrm{~m} / \mathrm{s}$

Figure 65 Iron-Oxide Particles Accumulation on Nozzle Side Walls
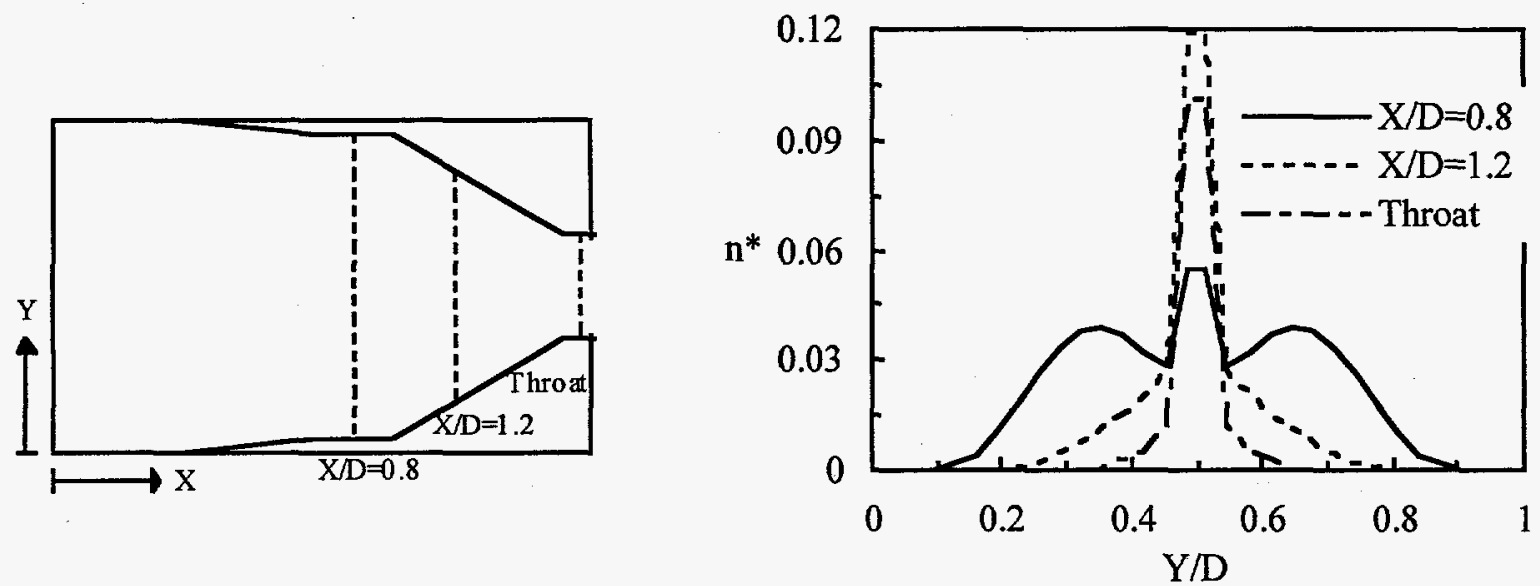

Figure 66 Development of Particle Number Density in Nozzle \#1

Figure 67 compares the number density profiles of iron-oxide particles at the nozzle throat for two different particle sizes (60 and $15 \mu \mathrm{m}$ ) and two different injection velocities (30 and $5 \mathrm{~m} / \mathrm{s}$ ). For easy comparison, the number density in the figure is normalized by the centerline number density. The reference particle size is $60 \mu \mathrm{m}$, and the reference injection velocity is $30 \mathrm{~m} / \mathrm{s}$. In Fig. 67, the solid line represents the reference result, the dotted line represents the $15-\mu \mathrm{m}$ and $30-\mathrm{m} / \mathrm{s}$ results, and the broken line represents the $60-\mu \mathrm{m}$ and $5-\mathrm{m} / \mathrm{s}$ results. As shown in the figure, particles with smaller diameter or lower injection velocity have better dispersion and a more uniform distribution at the nozzle throat. 


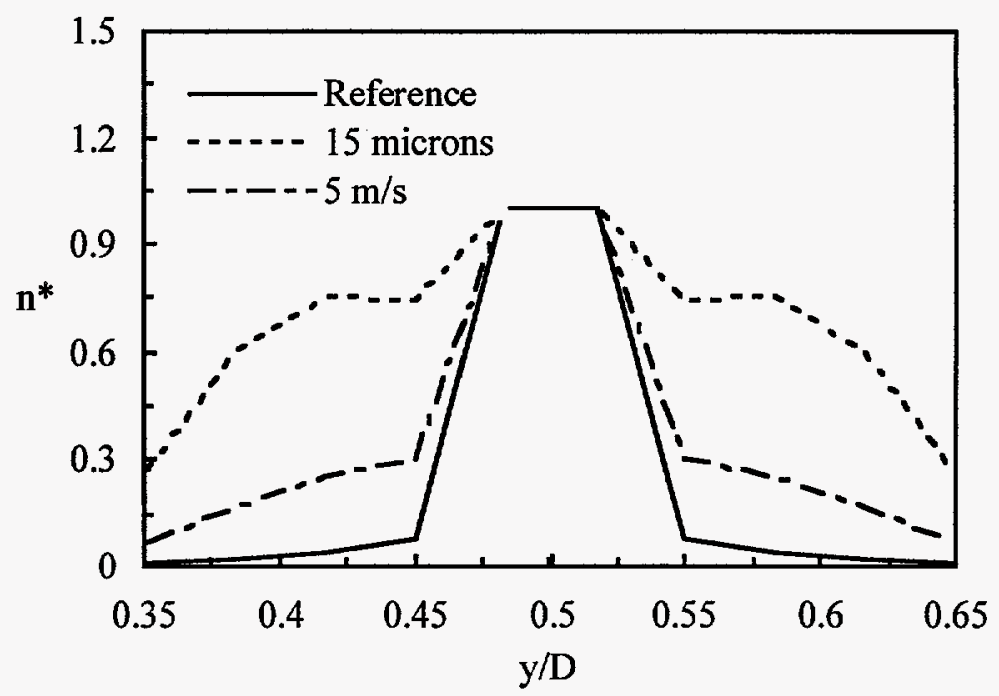

Figure 67 Effects of Particle Size and Injection Velocity on the Uniformity of the Particle Dispersion

Figure 68 compares the particle/droplet temperature profiles at the nozzle throat for two particle diameters, 30 and $60 \mu \mathrm{m}$. In the figure, the temperature is normalized by the inlet gas temperature. The solid line in the figure indicates the melting temperature $(0.65)$ for the iron oxide. Results indicate the $60-\mu \mathrm{m}$ particles are in solid state, and most $30-\mu \mathrm{m}$ particles are in liquid state.

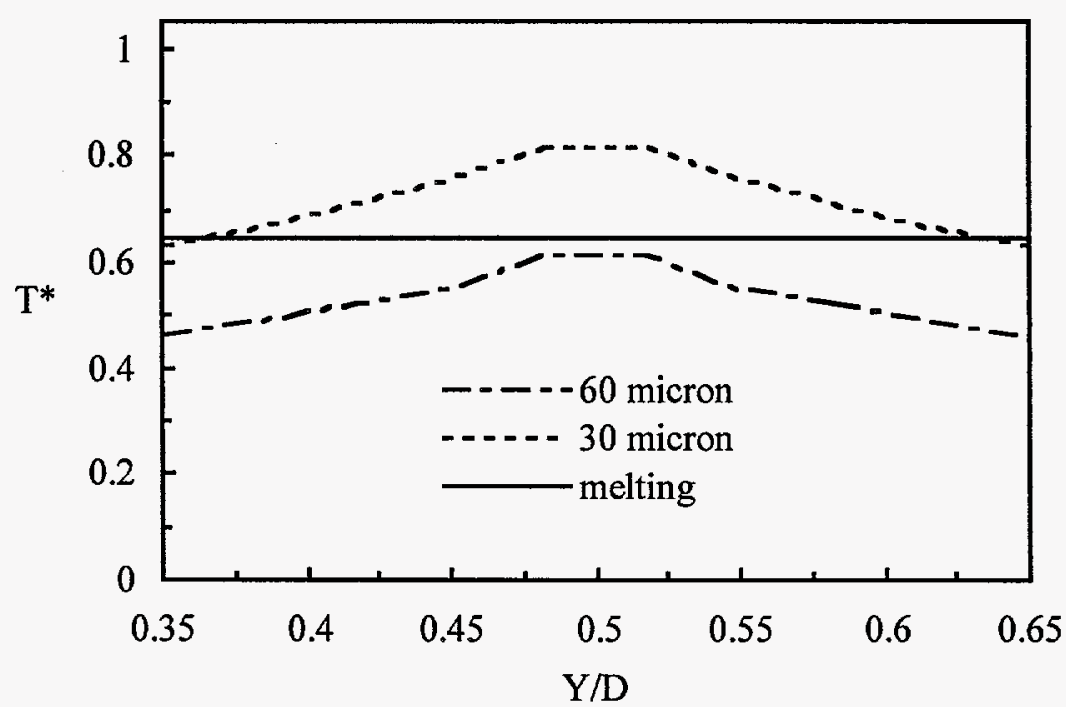

Figure 68 Effects of Particle Size on the Particle Temperature at the Nozzle Throat 


\section{ANALYSIS OF MHD CHANNEL OPERATION}

A high-temperature plasma flow is produced in the combustors (first and second stages). The plasma flow is accelerated in a nozzle before entering the MHD channel. The hightemperature, high-velocity plasma flows through the high magnetic field of the channel. The electromagnetic interaction generates electric power. ANL used a multigrid magnetohydrodynamic (MGMHD) computer code to simulate the plasma flow in the MHD channel [30]. The objective of the simulation was to investigate the effects of nonuniform flow patterns and slag leakage on the channel performance.

\subsection{Three-Dimensional Code (MGMHD) for MHD Generators and Diffusers}

The MGMHD computer code was developed at ANL in 1989 for the analyses of magnetohydrodynamic generators and diffusers [31]. This FORTRAN code solves the threedimensional partial differential equations for flow and electrical fields and incorporates an advanced multigrid solution algorithm. The magnetohydrodynamic equation set comprises the mass continuity equation, three momentum equations, the energy equation, two turbulence equations, and Maxwell's electrical equations. Turbulence is represented by a two-equation model in which partial differential equations are solved for the turbulent kinetic energy and its dissipation rate. Two unique features are provided in the MGMHD code: (1) a full approximation storage (FAS), block-implicit multigrid, finite-difference solution procedure for the cross-stream hydrodynamic equations, and (2) an FAS multigrid finite-difference solution procedure for the cross-steam electrical potential equation. Detailed description of the equations, solution procedures, options available to the user, and input instructions have been reported by Bouillard, et al. [31] Numerical solutions of three sample problems (two Faraday generators and a diagonally connected generator) were discussed. For two of these problems, the advantages of the multigrid algorithm were compared in terms of computational speed and solution accuracy.

The MGMHD code describes the interaction of a magnetic field with an electrically conducting gas in an MHD generator producing three-dimensional flow phenomena that manifest themselves in many forms [32-35]. In the boundary layers, velocity overshoots result directly from the cross-sectional nonuniformity of the axial component of the Lorentz force $\left(\mathrm{J}_{\mathrm{y}} \cdot \mathrm{B}\right)$. Flow asymmetries produced by secondary flows are generated by the nonuniformity in the magnetic field direction of the Lorentz force due to Hall current $\left(\mathrm{J}_{\mathbf{z}} \cdot \mathrm{B}\right)$. The threedimensional nature of electrical current and conductivity distributions introduces a nonuniform Joulean dissipation that distorts the flow and temperature fields. These distortions of the flow and temperature fields are further enhanced by the mutual coupling of these two fields with the electrical field. The extent and nature of these field distortions depend mainly on the channel electrical loading (Faraday or diagonal) and the channel type (conducting or insulating side walls). 
For single-grid solution methods of the Navier-Stokes and Maxwell's equations, the convergence rate becomes very slow because one grid is being used to resolve errors of various frequencies. Multigrid solution algorithms achieve rapid convergence by cycling between a fine grid and a series of coarser grids, resolving errors of different frequencies on those grids where the corresponding convergence rates are optimal. The MGMHD code can simulate supersonic and subsonic Faraday and diagonal generators for which the conductive plasma is generated by combustion of oil, gas, seed. Originally, slag effects on the channel walls were not considered in the MGMHD computer code. However, when attempts to simulate the MHD channel at CDIF in Butte, Montana, were made, it became clear that slag effects on the channel performance were critically important. It was, therefore, decided to incorporate a slag model into the MGMHD code to describe the influence of slag effects on the channel performance.

\subsection{Channel Slag Leakage Model}

Electrical power generated in the topping cycle tests at CDIF generally exhibited power levels well below the expected theoretical values. The nominal value of the power generated by the CDIF channel was expected to be about $1.5 \mathrm{MWe}$, while the power extracted during the latest tests performed in 1993 gave only 1.0-1.1 MWe for a whole range of loads.

Considerable disagreement exists within the MHD community as to the likely mechanisms of the power shortfall. Some argue for conductivity nonuniformity due to insufficient mixing length in combustor stages, while others claim evidence for current leakage through the slag layers on the channel wall. Power shortfall mechanisms are probably a combination of these two mechanisms. The combustor model described in Chapters 3, 4, and 5 of this report has addressed mixing nonuniformity in the second stage of the MHD combustor, which relates to the first part of the argument. In this section, the focus is on the second part of the argument, namely the effect of the slag layer on the performance of the generator.

As slag coats the cathodes, electrical shorting results, as shown in Fig. 69. In the figure, $\mathrm{N}_{\mathrm{s}}$ is the number of shorted electrodes; $P_{t}$ is the electrode pitch; $J_{x}$ and $J_{y}$ are $x$ - and $y$-current densities; and $\mathrm{H}$ is the height between anodes and cathodes. The shorted insulator gaps are caused by slag polarization, which can extend over several electrode pitches. This shorting across gaps results in the total Hall voltage being sustained by only a few electrode clusters, hence degrading the performance of the channel. To avoid massive electrode shorting, iron oxide is injected into the plasma flow in the converging nozzle to resegment the electrodes and eliminate shorted insulating gaps, but in practice a few clusters of shorted electrodes still exist. 


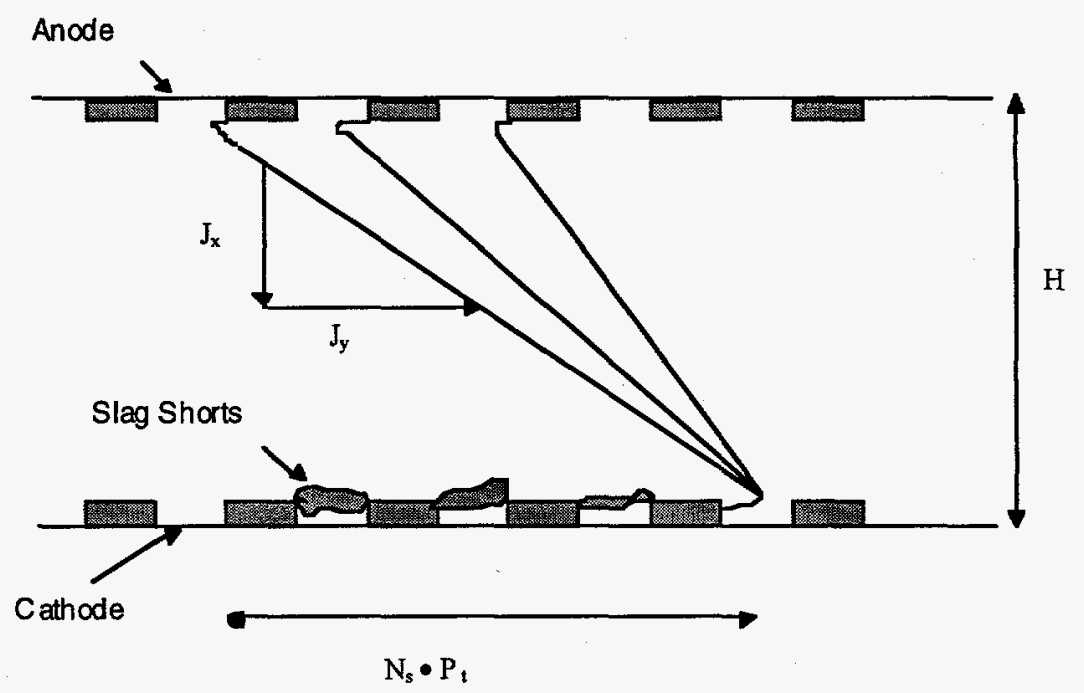

Figure 69 Finite Segmenting Effects by Shorted Cathode Gaps

Based on these observations, Rosa has identified [36-38] the following power shortfall mechanisms resulting from the slag layer:

(a) Slag resegmentation mechanism due to iron oxide (described above), and

(b) Resistive leak between electrode window frames.

For example, inferred leakage currents in the CDIF channel have been estimated to be about $100 \mathrm{~A}$. For a typical axial voltage of $6000 \mathrm{~V}$, this current leakage would represent an electrical power loss of $0.6 \mathrm{MWe}$, or a $40 \%$ power shortfall.

If the shorted intercathode gaps resegment into clusters of size $\mathrm{N}_{s} \mathrm{P}_{t}$, the resulting axial current density (i.e., Hall current) in the case of a Faraday connection can be modeled as

$$
\left\langle J_{z}\right\rangle=\frac{N_{s} P_{t}}{H g t}\left\langle J_{y}\right\rangle
$$

and for a diagonal connection as:

$$
\left\langle J_{z}\right\rangle=\frac{I_{l}}{A}+t\left\langle J_{y}\right\rangle+\frac{N_{s} P_{t}}{H g t}\left\langle J_{y}\right\rangle
$$

or

$$
\left\langle J_{z}\right\rangle=\frac{I_{l}}{A}+\left\langle J_{y}\right\rangle\left[t+\frac{N_{s} P_{t}}{H g t}\right]
$$

where $t$ is the tangent of the link overlap angle and $A$ is cross-sectional area. As can be seen, when the number of clustered gaps increases, the channel operates at an effective diagonalization angle that can be significantly different from the nominal one and lead to off-design regimes. 
In addition, the slag introduces some resistive links between the frames of the generator, and a value of about $1 \mathrm{ohm}$ has been identified by Pollina [39]. For a Faraday generator, an extra Hall current leakage results from these interframe resistances, which can be expressed as

$$
\left\langle J_{z}\right\rangle=\frac{-E_{z} P_{t}}{A \cdot R_{l k}}
$$

where $R_{1 k}$ is the interframe resistance due to slag (which needs to be measured). For a diagonal generator, the load current density, $\mathrm{J}_{1}$, can be expressed as

$$
J_{l}=\left\langle J_{z}\right\rangle-\left\langle J_{y}\right\rangle t-\frac{E_{z} P_{t}}{A \cdot R_{l k}}
$$

The current leakage due to the inter-electrode resistance is mainly a function of the Hall electric field in the diagonal model, Eq. 7.5, whereas the current leakage due to the cathode resegmentation is mainly a function of the Faraday current in the Faraday mode, Eq. 7.1. This trend was experimentally observed by Rosa et al. [36-38].

The slag and shorted cathodes have an effect to modify the Hall electric field. The Hall electric field for a Faraday channel when slag leakage is accounted for is given by

$$
E_{z}=\frac{\left\langle\sigma_{n \beta} \phi_{2, y}\right\rangle+\frac{N_{s} P_{t}}{H g t}\left\langle\sigma_{n} \phi_{2, y}\right\rangle+B\left[\frac{N_{s} P_{t}}{H g t}\left\langle\sigma_{n}(w-\beta u)+\left\langle\sigma_{n}(\beta w+u\rangle\right\rangle\right]\right.}{\left\langle\sigma_{n}\right\rangle+\left\langle\sigma_{n} \beta \beta_{\phi 1, y}\right\rangle-\frac{N_{s} P_{t}}{H g t}\left(\left\langle\beta \sigma_{n}\right\rangle-\left\langle\sigma_{n} \phi_{1, y}\right\rangle\right)+\frac{P_{t}}{A \cdot R_{\text {leak }}}}
$$

where $\mathrm{N}_{\mathrm{s}}$ is the number of shorted cathodes. (For $\mathrm{N}_{\mathrm{s}}=0$, the usual expression for $\mathrm{E}_{\mathrm{z}}$ results.) For a diagonal channel, the Hall electric field is given by:

$$
E_{z}=\frac{J_{1}-\left\langle\sigma_{n}\left(\beta+\cot (\gamma)+\frac{N_{s} P_{t}}{H g t}\right)\left(\phi_{2, y}+u B\right)\right\rangle-B\left\langle\sigma_{n}\left(1-\beta\left(\cos (\gamma)+\frac{N_{s} P_{t}}{H g t}\right)\right) \nu\right\rangle}{\left(1+\cot (\gamma)\left(\cos (\gamma)+\frac{N_{s} P_{t}}{H g t}\right)\right)\left\langle\sigma_{n}\right\rangle-\frac{N_{s} P_{t}}{H g t}\left\langle\beta \sigma_{n}\right\rangle+\left\langle\sigma_{n}\left(\beta+\cot (\gamma)+\frac{N_{s} P_{t}}{H g t}\right)\left(\phi_{1, y}\right)\right\rangle+\frac{P_{t}}{A \cdot R_{\text {leak }}}}
$$

where the total electric potential $\psi$ is expressed as

$$
\psi=-[z-y \bullet \cot (\gamma)] E_{z}+\phi(x, y)
$$

with

$$
\phi(u, y)=E_{z} \phi_{1}(x, y)+\phi_{2}(x, y)
$$


The shorted cathode groups increase the Hall parameter. This slag leakage model is very similar to that successfully used by Lineberry et al. [40].

\subsection{CDIF Channel Description}

The CDIF- $1 \mathrm{~A}_{4}$ channel was designed to have a nominal power output of $1.5 \mathrm{MWe}$ and a lifetime of 2000 hours for very harsh MHD operating environments. A description of the $1 \mathrm{~A}_{4}$ channel, nozzle, and diffuser as they sit in the magnet bore is given by S.W. Petty et al. [41], and details of the design and fabrication with drawings can be found in that reference. A brief description of the MHD channel geometry along with the features relevant to numerical modeling and simulation of channel processes is provided in the following paragraphs.

The $1 \mathrm{~A}_{4}$ channel is designed for supersonic flow and is basically integrated into the diverging section of a supersonic nozzle downstream from the combustor system. The MHD channel is $4.8 \mathrm{~m}$ long with a rectangular cross section. The top and bottom walls are parallel, and the side walls diverge linearly to allow supersonic expansion of the plasma flow in the channel at a short distance beyond the nozzle throat. A diagram of the MHD nozzle/channel system is shown in Fig. 70, which includes the MHD converging nozzle and diverging channel with their relative positions within the system. Both anodes and cathodes are water cooled, with the anodes being incorporated into the upper wall, and the cathodes into the lower wall, so the anode to cathode distance remains constant along the channel length. Details of the channel dimensions and operating conditions are given in Table 15.

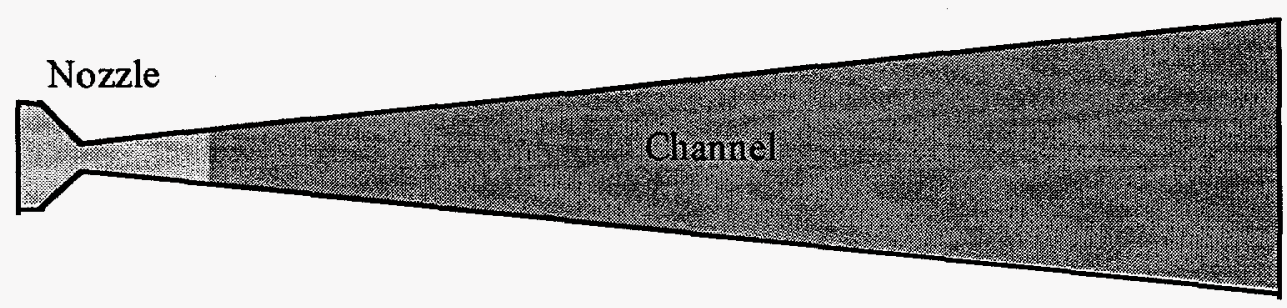

Top View

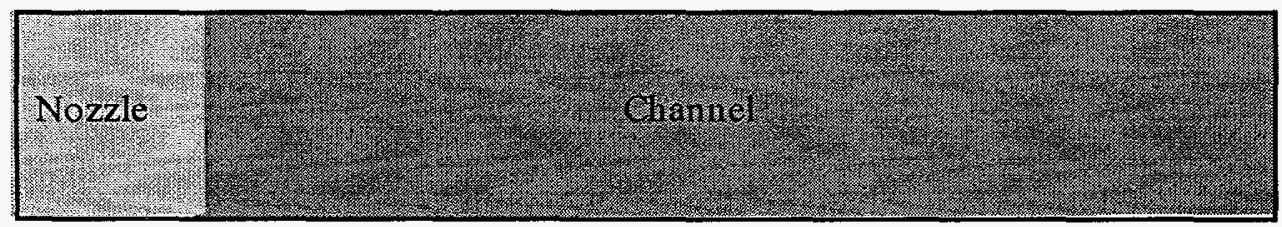

Side-View

Figure 70 Typical CDIF $1 \mathrm{~A}_{4}$ Nozzle and Channel Assembly 
Table 15 Typical $1 \mathrm{~A}_{4}$ Channel Parameters

\begin{tabular}{|l|c|}
\hline \multicolumn{1}{|c|}{ Channel Type } & $\begin{array}{c}\text { Supersonic with } \\
\text { Segmented Diagonal Wall }\end{array}$ \\
\hline Channel Loading & Diagonal \\
\hline Nominal Inlet Operating Pressure (atm) & 6 \\
\hline Number of Electrodes (pairs) & 245 \\
\hline Diagonal Connection Overlap & 12 \\
Electrodes & 36 \\
Degrees & $20-40$ \\
\hline Electrode Current Range (A) & $35-75$ \\
\hline Interelectrode Voltage Range (V) & 1.5 \\
\hline Power Output (MWe) & 6,200 \\
\hline Load Voltage (V) & 240 \\
\hline Load Current (A) & $0.23 \times 0.18$ \\
\hline Inlet Dimensions (nozzle) $(\mathrm{H} \mathrm{x} \mathrm{W)(m)}$ & $0.23 \times 0.068$ \\
\hline Throat Dimensions (nozzle) $(\mathrm{H} \mathrm{x} \mathrm{W})(\mathrm{m})$ & $0.23 \times 0.33$ \\
\hline Channel Outlet Dimensions $(\mathrm{H} \mathrm{x} \mathrm{W})(\mathrm{m})$ & 4.8 \\
\hline Length (nozzle and channel) $(\mathrm{m})$ & 4 \\
\hline Heat Loss (nozzle/channel) $(\mathrm{MW})$ & \\
\hline
\end{tabular}

Satisfactory performance of the anode wall depends on slag coverage on the gas-side surface. Therefore the anode was designed with grooves between adjacent anode caps that have been spaced and staggered to provide footholds for slag attachment.

The channel divergence is provided entirely by the top and bottom electrode walls. The side wall elements are made of segmented Z-bars. High voltage arcing gaps can develop on the cathode wall because of slag polarization. These high voltages also impact on the side walls, accelerating their electrochemical and arc erosion processes. Tungsten capping is requisite for durability of these elements in the presence of these high-voltage arcing gaps.

In the tests conducted to date, the iron-oxide injection onto the cathode wall has, to a large extent, mitigated these high-voltage arcing gaps. However, only limited testing with iron-oxide slurry injection has been completed. Computer modeling and analysis conducted at ANL to identify and help rectify problem areas to achieve a good iron-oxide coating have been discussed in Chapter 6. 
Table 161 A Generator Operating Conditions

\begin{tabular}{|l|r|}
\hline Inlet Mach No. & 1.5 \\
\hline Thermal throughput $(\mathrm{MW})$ & 50 \\
\hline Inlet conductivity $(\mathrm{S} / \mathrm{m})$ & $7-9$ \\
\hline Total mass flow $(\mathrm{kg} / \mathrm{s})$ & 10.26 \\
\hline Nominal iron oxide injection rate $(\mathrm{kg} / \mathrm{min})$ & $0.9-1.8$ \\
\hline
\end{tabular}

The generator was designed to operate at nominal electrode currents of about 20-40 A and nominal inter-electrode voltages of about 35-70 V. Due to local shorting of the electrodes, it was experimentally found that intercathode voltages could reach up to $120 \mathrm{~V}$. The typical operating conditions relevant to the MGMHD computer simulation are listed in Table 16, with the operating conditions for the fuel/reactant/seed listed in Table 17 for the CDIF-Test-93-DVT-06.

Table 17 Typical Fuel/Reactant/Seed Compositions at CDIF [42]

\begin{tabular}{|l|c|}
\hline Coal composition (dry basis) & Value \\
\hline$\% \mathrm{C}$ & 69.22 \\
\hline$\% \mathrm{H}$ & 4.61 \\
\hline$\% \mathrm{~S}$ & 0.79 \\
\hline$\% \mathrm{~N}$ & 1.16 \\
\hline$\% \mathrm{O}$ & 12.7 \\
\hline Coal moisture $(\%)$ & 11 \\
\hline Coal higher heating value $(\mathrm{cal} / \mathrm{g})$ & 6372 \\
\hline Fuel flow rate $(\mathrm{kg} / \mathrm{s})$ & 1.9 \\
\hline Oxygen flow rate $(\mathrm{kg} / \mathrm{s})$ & 3.82 \\
\hline Nitrogen flow rate $(\mathrm{kg} / \mathrm{s})$ & 4.27 \\
\hline Seed flow rate $\left(\mathrm{K}_{2} \mathrm{CO}{ }_{3}\right)(\mathrm{kg} / \mathrm{s})$ & 0.27 \\
\hline Total flow rate $(\mathrm{kg} / \mathrm{s})$ & 10.26 \\
\hline
\end{tabular}

To obtain commercially viable levels of electrical energy, powerful magnetic fields on the order of 2-6 $\mathrm{T}$ are required. At CDIF, the magnet used is a conventional (i.e., not superconducting) electromagnet, which has resistive windings on an iron core. It delivers peak fields on the order of $3 \mathrm{~T}$, with field axial distribution represented in Fig. 71. The active length of the magnet is about $4.2 \mathrm{~m}$, which is also about the longitudinal size of the channel. 


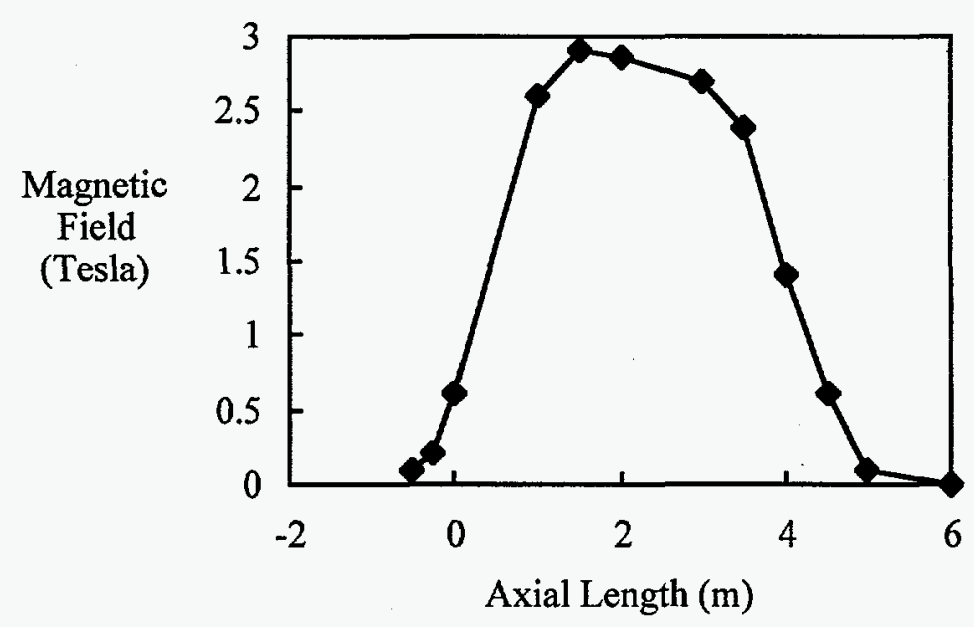

Figure 71 CDIF Axial Magnetic Field Distribution

\subsection{CDIF Slagging Diagonal Channel Simulation}

To illustrate the application of the MGMHD computer code to the analysis of a supersonic and slagging diagonal channel, two simulations were performed: (1) a simulation with no MHD loading, and (2) a simulation with MHD loading. These two simulations have been tested and compared with experimental data collected on the $1 \mathrm{~A}_{4} \mathrm{MHD}$ generator of the DOE-sponsored CDIF experimental facility located in Butte, Montana.

First, an unpowered channel configuration of the CDIF supersonic channel was simulated. An unpowered configuration means that the plasma is being fired by the combustor into the channel, but neither electric nor magnetic loadings are imposed on the channel to produce electricity. For this simulation, the plasma properties were computed with the coal/reactant/seed compositions summarized in Table 18 (test 93-DVT-06 at CDIF [42]).

A slag temperature of about $1700 \mathrm{~K}$ was assumed in the computations. The condensation temperature of the slag commonly ranged between 1600 and $1800 \mathrm{~K}$. The predicted heat loss through the channel walls was about $4 \mathrm{MW}$. CDIF reported a measured heat loss of about 3.9 MW for the test 93-DVT-27 [42], which is in close agreement with the predicted results.

The prediction of the axial distribution of the pressure along the channel length is represented in Fig. 72. The predicted pressure decreases from $3.5 \mathrm{~atm}$ to a subatmospheric value $(0.5 \mathrm{~atm})$ at the end of the channel. The computed axial pressure profile compares well with the experimental data. The pressure decreases because of the supersonic expansion of the plasma. Note that if the plasma were an incompressible fluid, the expansion would lead to a pressure increase and a velocity decrease. In supersonic regimes, the converse is true, with the velocity or Mach number increasing with the channel expansion as shown in Fig. 73. The velocity entering the channel is about $1000 \mathrm{~m} / \mathrm{s}$, which is the choking flow condition at the nozzle throat where the 
Mach number is one. Due to this supersonic expansion, the temperature of the plasma drops significantly along the channel length, as shown in Fig. 73. This figure shows the temperature of the plasma drops from $2700 \mathrm{~K}$ at the channel inlet to about $1850 \mathrm{~K}$ at the channel exit.

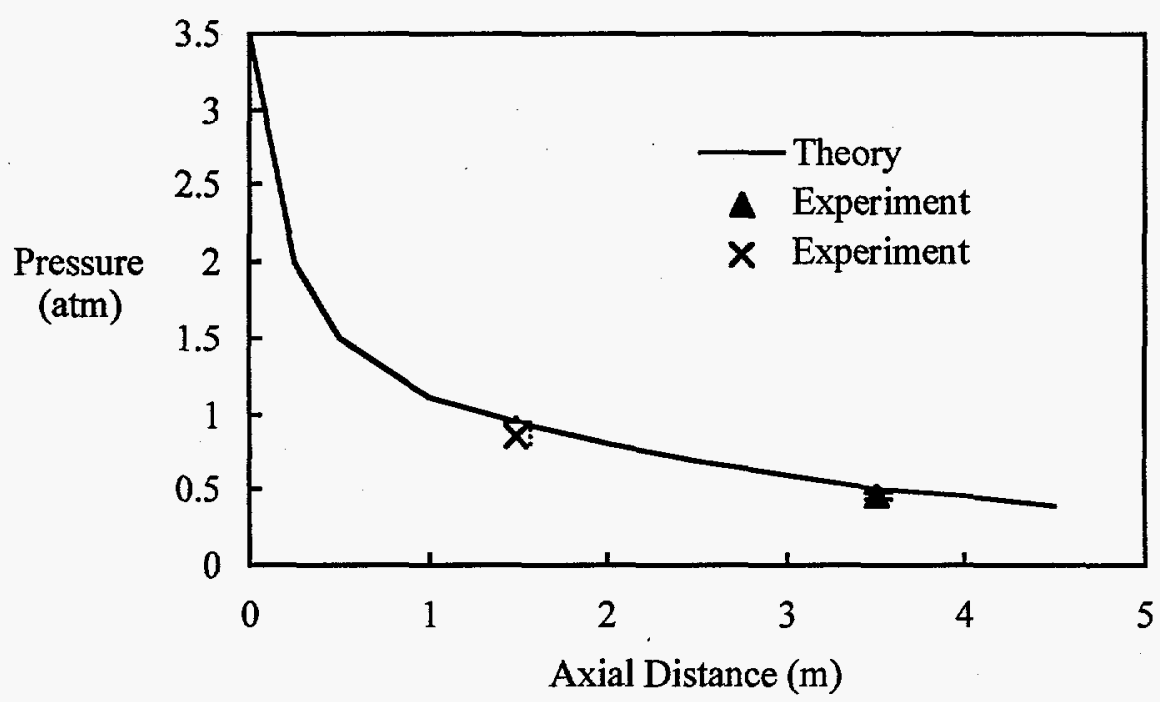

Figure 72 Computed and Measured Axial Pressure Distribution (Unpowered Case)

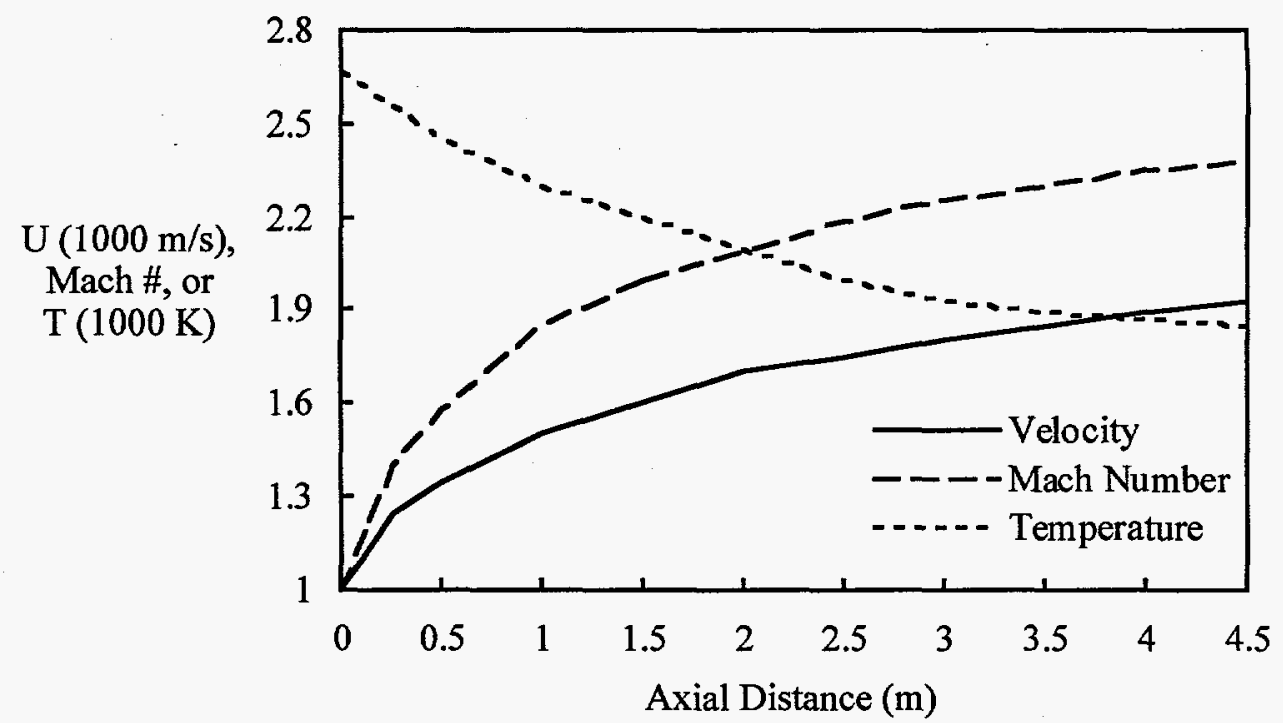

Figure 73 Calculated Axial Central Velocity, Temperature, and Mach Number 
At $3.5 \mathrm{~m}$ from the nozzle into the channel, a nonuniform cross-section temperature profile develops, as shown in Fig. 74. Such two-dimensional temperature plots clearly indicate the existence of a strong cooling phenomenon in the core area of the channel that is produced by the supersonic expansion. Also indicated is the excessive viscous heating of the plasma in the channel wall regions. Such a detailed channel flow behavior has not been reported previously, because no systematic three-dimensional analysis had been performed for this channel configuration. In the past, a turbulent temperature profile was usually assumed, with larger conductivity residing in the core area and lower conductivity near the wall. In fact, the reality is the opposite, with the highest conductivity near the wall and the lowest in the core, as shown in Fig. 75 . At $3.5 \mathrm{~m}$ from the nozzle throat into the channel, the conductivity peaks to about $13.4 \mathrm{~S} / \mathrm{m}$ in the corners of the side walls and the electrode walls of the channel, while the central value of the conductivity drops to almost zero. The central conductivity axial profile has been plotted in Fig. 76; the central axial conductvity decreases rapidly from $8 \mathrm{~S} / \mathrm{m}$ to less than $1 \mathrm{~S} / \mathrm{m}$ in the first $2 \mathrm{~m}$ of the channel.

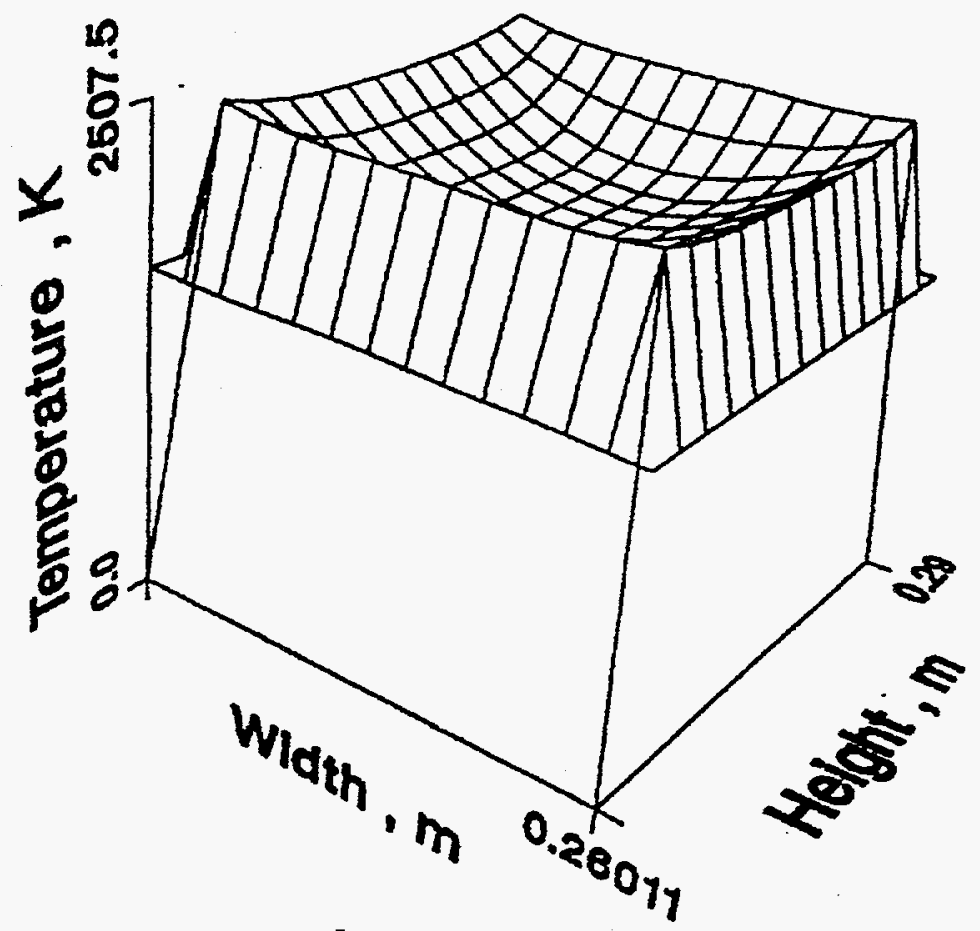

Figure 74 Temperature Profile on a Cross Section $3.5 \mathrm{~m}$ from the Channel Inlet 


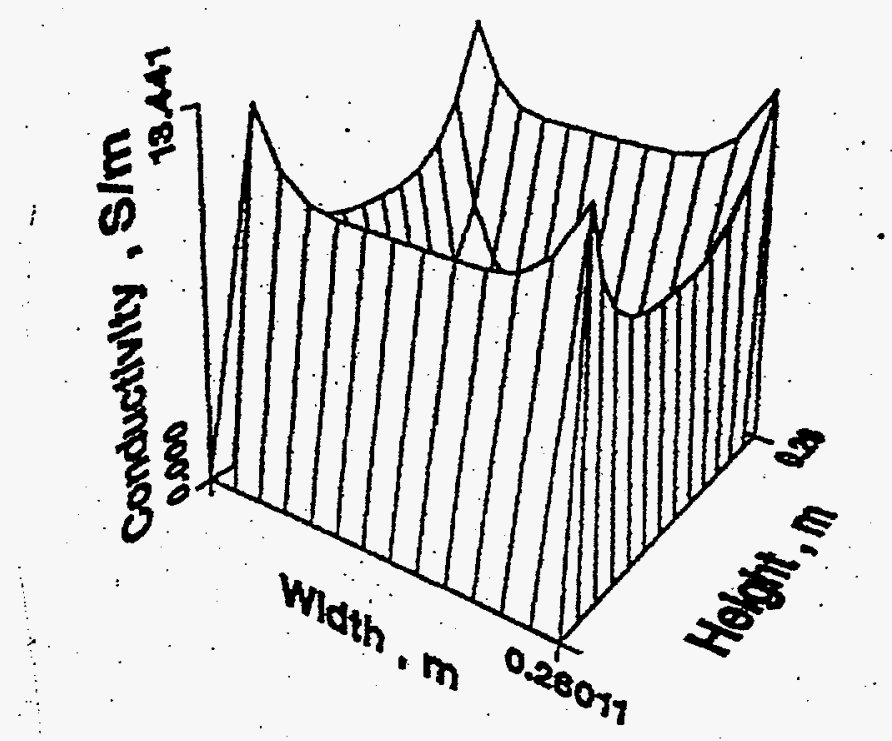

Figure 75 Conductivity Profile on a Cross Section $3.5 \mathrm{~m}$ from the Channel Inlet

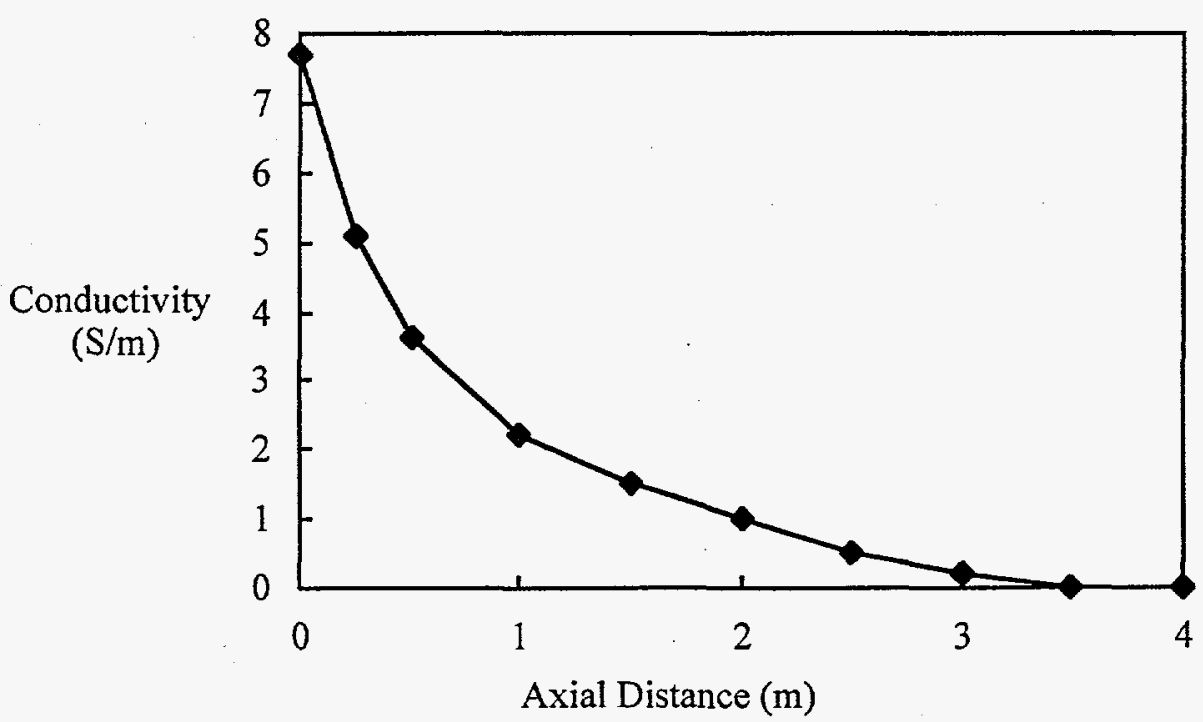

Figure 76 Computed Central Conductivity in the Axial Direction 
These predictions have three important implications for the operation and the design of the channel:

(1) About $50 \%$ of the channel length is not effectively utilized because of the strong plasma cooling by supersonic expansion. This would tend to reduce the nominal power output of the channel. Perhaps a subsonic or low-expansion ratio operating mode would be more beneficial.

(2) The nonuniformity of the conductivity creates larger current densities near the wall, where the conductivity is highest. This condition will potentially lead to serious erosion/corrosion damage to the electrodes and the side walls (Z-bars). This phenomenon has been experimentally observed during the operation of the $1 \mathrm{~A}_{4}$ channel.

(3) A scaled-up version of this channel would perform very poorly, since the major central part of the channel would be underutilized.

Clearly, three-dimensional effects have a significant impact on the performance of the MHD generator component of the MHD power generation system, and the three-dimensional CFD code used here provides the capability to qualitatively and quantitatively assess the relative magnitude and impact of these effects on the performance of the generator.

The CDIF slagging diagonal supersonic channel was also simulated for various electric loads. This simulation included the computation of the characteristic lines of the generator in slagging and nonslagging regimes.

The currents and voltages in the MHD generator are functions of the external resistive load of the channel. The external resistive load imposes the relation between the voltage and the current that develops across the machine. The generator itself has a set of performance characteristics that vary as a function of current and voltage but are independent of the external resistive loading. At the design operating conditions of a such a generator, the characteristic lines intersect with the load lines, as shown in Fig. 77. For the CDIF generator, the characteristic lines were predicted for both the nonslagging and slagging regimes using the MGMHD computer code.

Experimentally, the characteristic lines are determined by measuring the resulting voltages and currents while varying the load resistance. The predicted voltages and currents range between 0 and $11 \mathrm{kV}$ and 0 and $350 \mathrm{~A}$, respectively. At zero current load, the voltage obtained is called the open-circuit voltage, which is about $11 \mathrm{kV}$, while at zero voltage, the current that can be extracted from the MHD channel (about $350 \mathrm{~A}$ ) is called the short-circuit current. At these two conditions, the extracted electric power is zero. The predicted channel characteristics are similar for both the slagging and nonslagging cases, with the characteristic line shifting downward for the slagging regime. In fact, the electrical power that can be extracted from the 
channel operating in a slagging mode is lower than that from the nonslagging mode because of the loss of power through Joule electric heating in the slag layers. The computations indicate that the voltage and current can be reduced by as much as $1.5 \mathrm{kV}$ and $75-100 \mathrm{~A}$, respectively, by the presence of the slag, illustrating the profound impact of slag layers on channel performance.

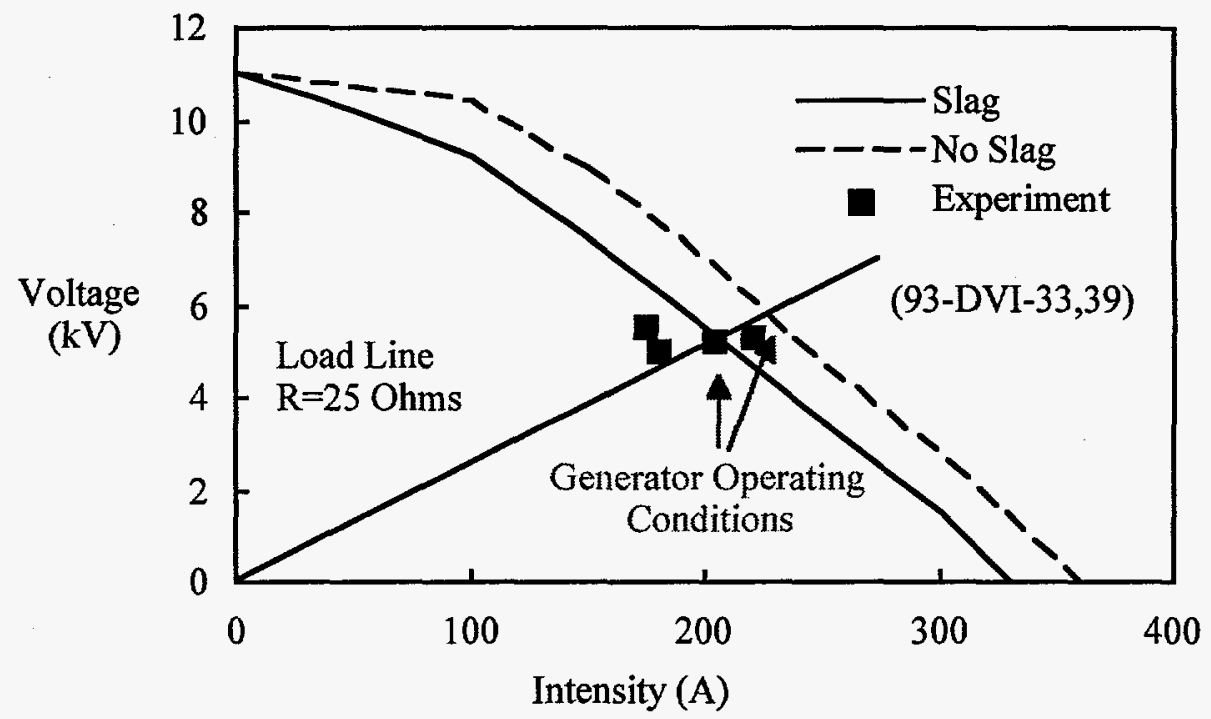

Figure 77 Slag Effect on the CDIF Generator Characteristics

These simulations also indicate that at low current $(<100 \mathrm{~A})$, the characteristics of the channel are nonlinear for both the slagging and nonslagging regimes. These effects are indicative of the development of strong nonlinear electric fields and current densities at current loads that may be directly related to the temperature and conductivity nonuniformity that develop in the channel as discussed in the previous paragraphs.

For illustration purposes, a resistive load line with $R=25$ ohms is plotted in Fig. 77. The product of the voltage and the current at the intersection point gives the electrical power output of the MHD channel. A few experimental values of voltages and currents of the channel run in slagging mode have been superimposed on this figure. Computed and measured voltages and currents agree very well, considering the fairly broad assumptions made in the model.

The CDIF test operation conditions revolve around a load current of about $200 \mathrm{~A}$ and a voltage of about $5.5 \mathrm{kV}$, which yield a power of about $1.1 \mathrm{MWe}$. The computed power output of the simulation is in very good agreement with the experimental data. The MHD channel was designed for $1.5 \mathrm{MWe}$ at nominal power, but the slagging effects reduced this power to $1.1 \mathrm{MWe}$, amounting to a $30 \%$ power shortfall. This power shortfall is illustrated in Fig. 78 as a function of the current load. Experiments performed at CDIF showed that the channel can deliver higher power outputs, namely $1.6 \mathrm{MWe}$, by boosting the oxygen ratio of the second-stage combustor, thus enhancing the plasma conductivity. However, results of the channel simulations 
show that even with a higher channel inlet temperature and consequently higher conductivity, the channel in its present configuration would still suffer from the large supersonic expansion and the resulting conductivity nonuniformity.

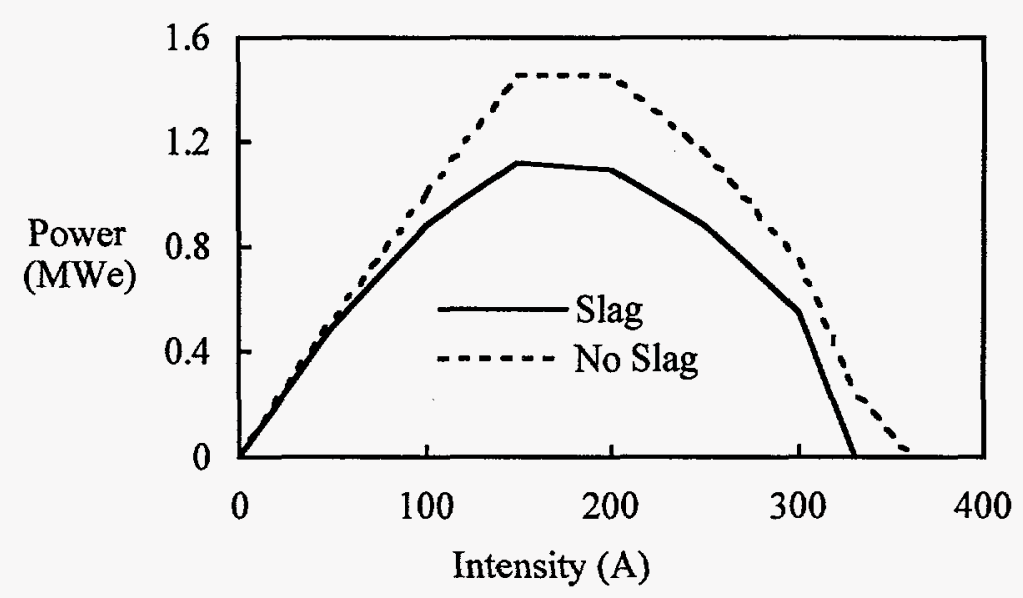

Figure 78 Slag Effect on the Electrical Power Output of the CDIF Generator

Data reduction of these simulations included the computation of extracted electric power and generator efficiency versus current loadings. Figure 78 shows the predicted power output of the CDIF supersonic channel for the slagging and nonslagging regimes over the whole range of current loads, while Fig. 79 displays the generator efficiency at the same operating conditions. At zero current and at short circuit current $(350 \mathrm{~A})$, the extracted electrical power is nearly zero. In the diagonal configuration of the channel (with diagonal angle of 36 degrees), the extracted power is maximal at about $200 \mathrm{~A}$, while the maximum efficiency is obtained at about $250 \mathrm{~A}$ (Fig. 79), where the extracted power is computed to be $1.13 \mathrm{MWe}$ and $0.97 \mathrm{MWe}$, respectively, for the nonslagging and slagging regimes. Note that these maxima occur roughly at the same load conditions for both the slagging and nonslagging channels and that the power extracted at the most efficient operating point (i.e., $250 \mathrm{~A}$ ) represents about $88 \%$ of the maximum power output attainable (i.e., at $200 \mathrm{~A}$ ). Hence, the most efficient operation of this machine is not at maximum power condition. 


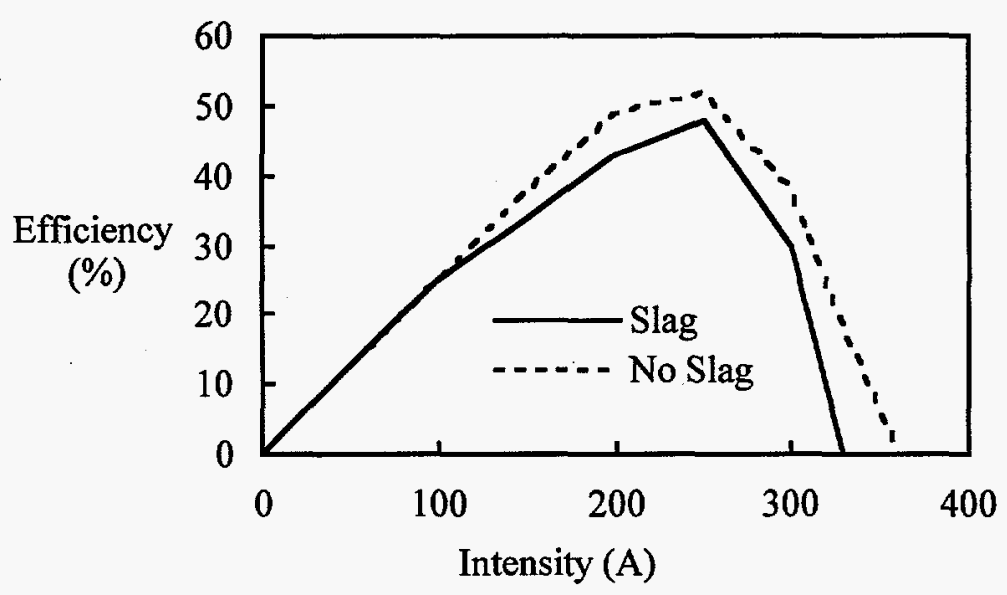

Figure 79 Slag Effect on the Electrical Efficiency of the CDIF Generator

To investigate the three-dimensional effects in more detail, three-dimensional current densities and cross-sectional velocity patterns were plotted and analyzed. Figures 80a and 80b show a two-dimensional current distribution in the central plane of the generator and in the boundary layer near the side walls, respectively, plotted along the channel length. These figures show large current densities near the side walls and low current densities in the channel core. This effect is the direct consequence of the nonuniform conductivity discussed in the previous sections. Hence, the bulk of the MHD phenomena take place near the walls. A minor MHD mixing effect occurs in the central portion of the channel, $2.2-\mathrm{m}$ downstream from the nozzle throat, as indicated in Fig. 81. MHD mixing by secondary flows is small because the Hall currents are small in the central core of the channel; MHD mixing is not strong enough to eliminate the significant temperature and conductivity nonuniformity present in the wall regions, as previously discussed. 


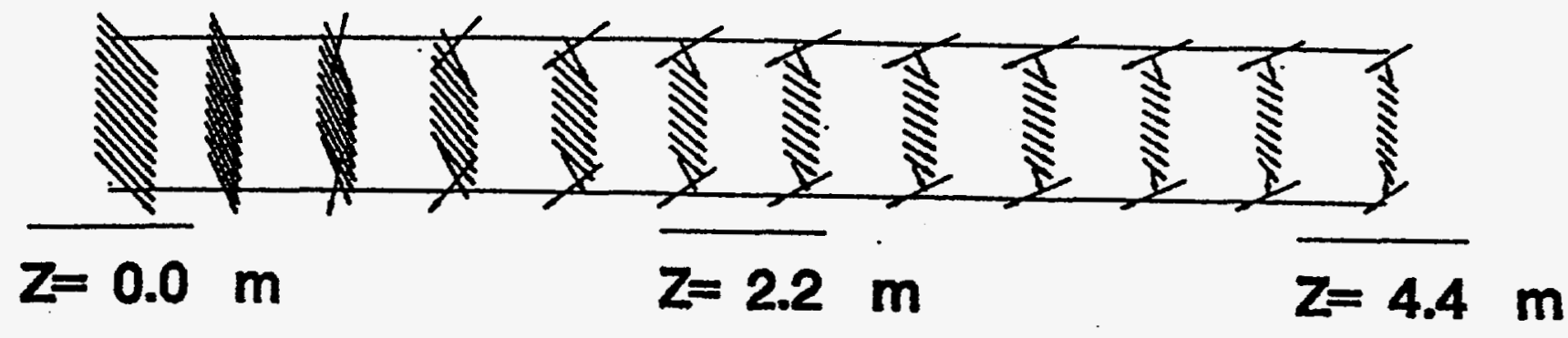

(a)

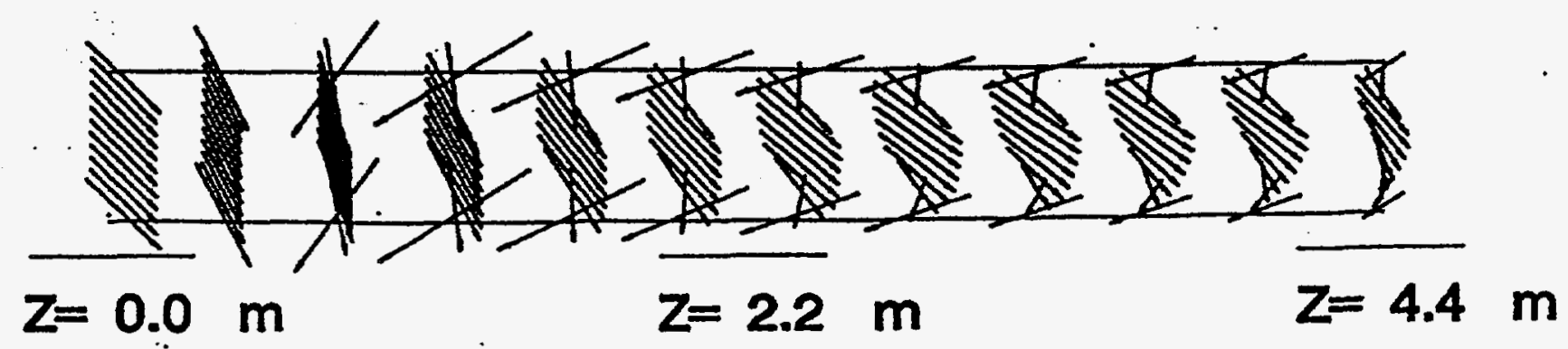

(b)

Figure 80 Computed Current Distribution of the CDIF Generator (a) in the Central Plane of the Generator and (b) in the Boundary Layer near the Side Wall 


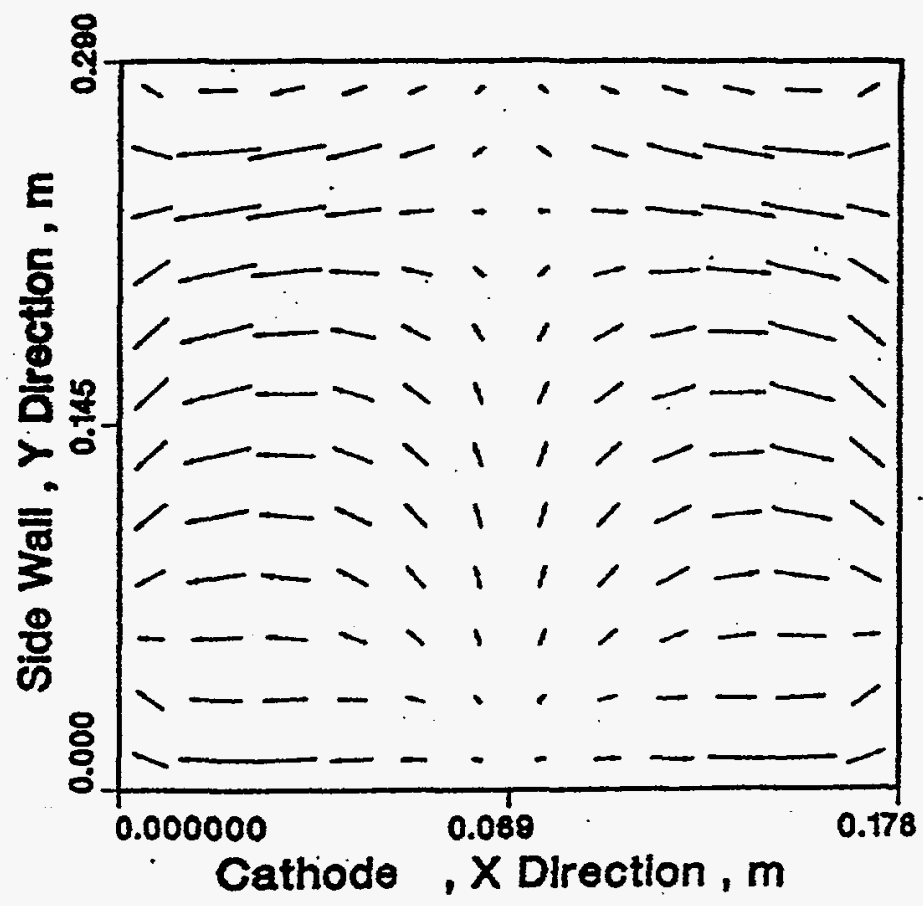

Figure 81 Velocity Profile on a Cross Section 2.2-m Downstream from the Nozzle Exit

Leakage currents, Hall voltages, Faraday potentials/current densities, and diagonal current leaks were also computed in the course of the MHD channel simulation, and some typical results are shown in Fig. 82. In the figure, the predicted leakage currents per pitch along the channel length are about $30 \mathrm{~A}$ per pitch. This current lies within the lower range of what Lineberry et al. assumed (40-100 A) in their study [40]. Note that these currents no longer need to be assumed in the computation of the MHD channel flow and associated MHD processes but are computed within the channel simulation by the MHD channel computer code. The computed leakage currents amount to an electrical power loss of about $0.165 \mathrm{MWe}$. A typical computed Hall voltage axial profile is shown in Fig. 82 and is seen to develop only after the first $0.6 \mathrm{~m}$ in the channel. The Hall voltage increases almost linearly along the channel length and the corresponding electric field is also plotted in Fig. 82. 


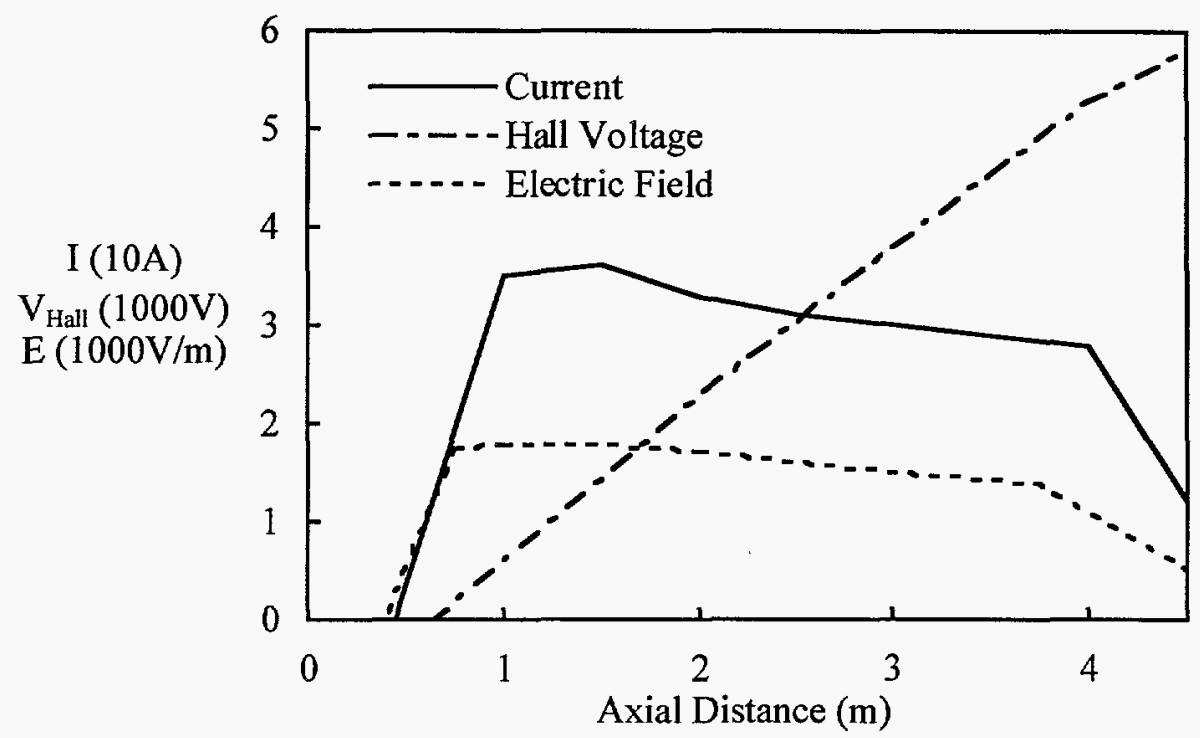

Figure 82 Computed Axial Distributions of Current Leakage, Hall Voltage, and Electric Field

In Figs. 83-85, computed results are compared with a corresponding CDIF experiment. The predicted diagonal link currents (about $15 \mathrm{~A}$ ) agree favorably with the experiment, and the Faraday potential and current densities are in good agreement with experimental data.

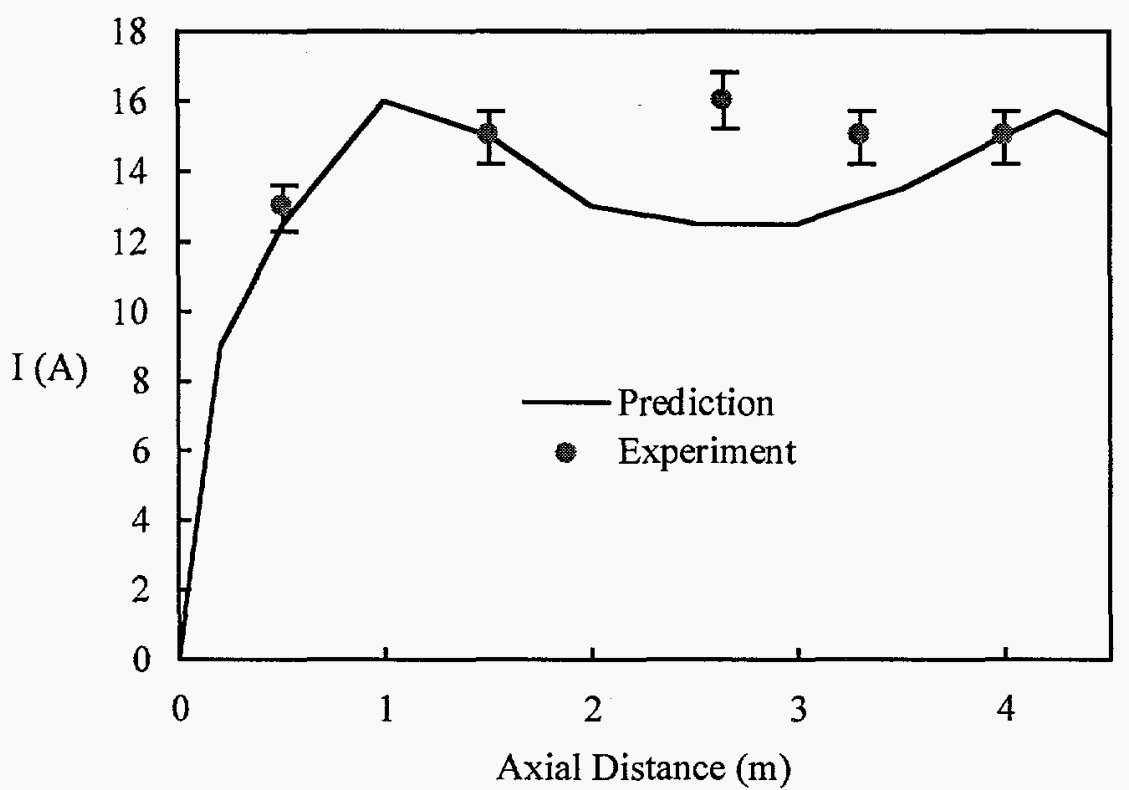

Figure 83 Computed and Measured Axial Distributions of Faraday Current Intensity 


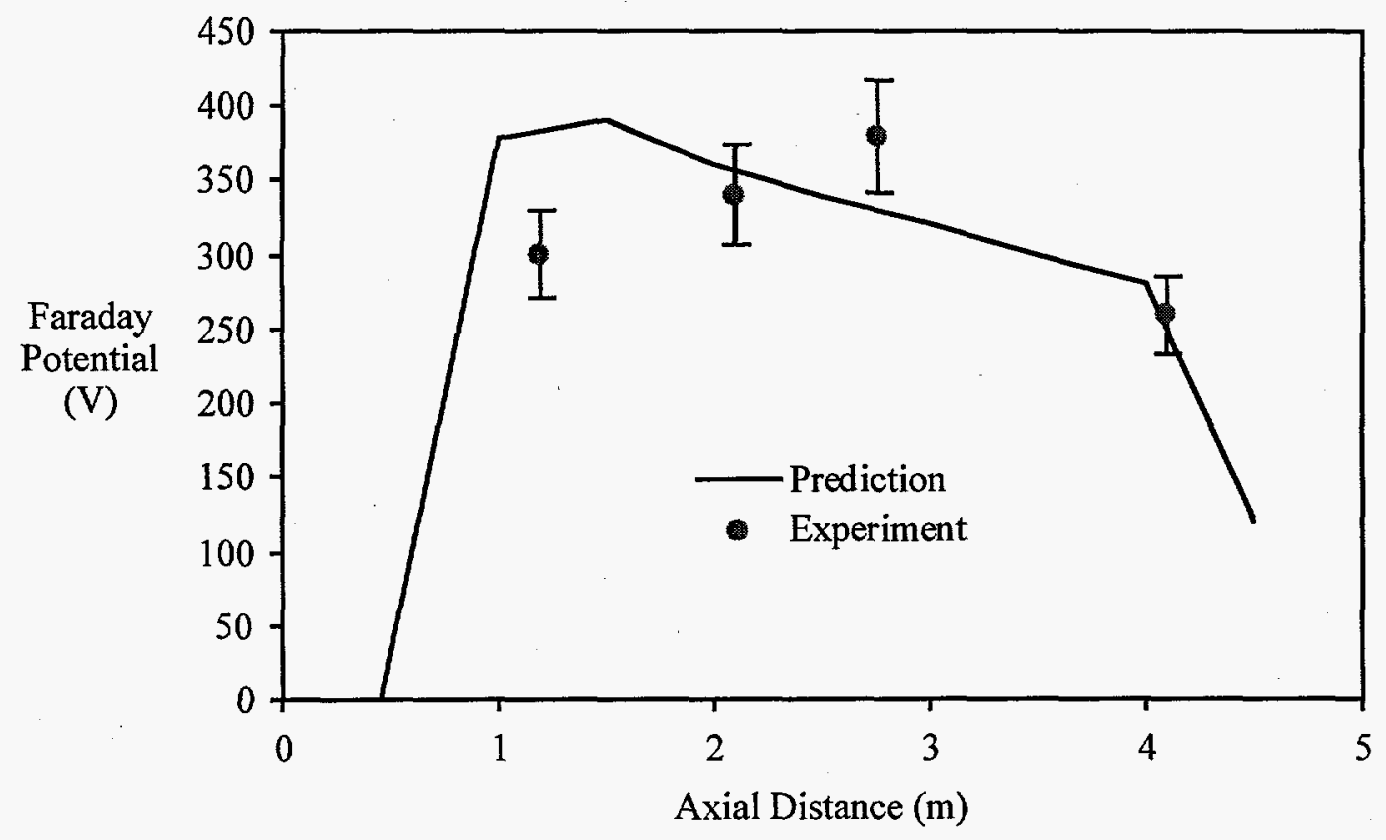

Figure 84 Computed and Measured Axial Distributions of Faraday Potential

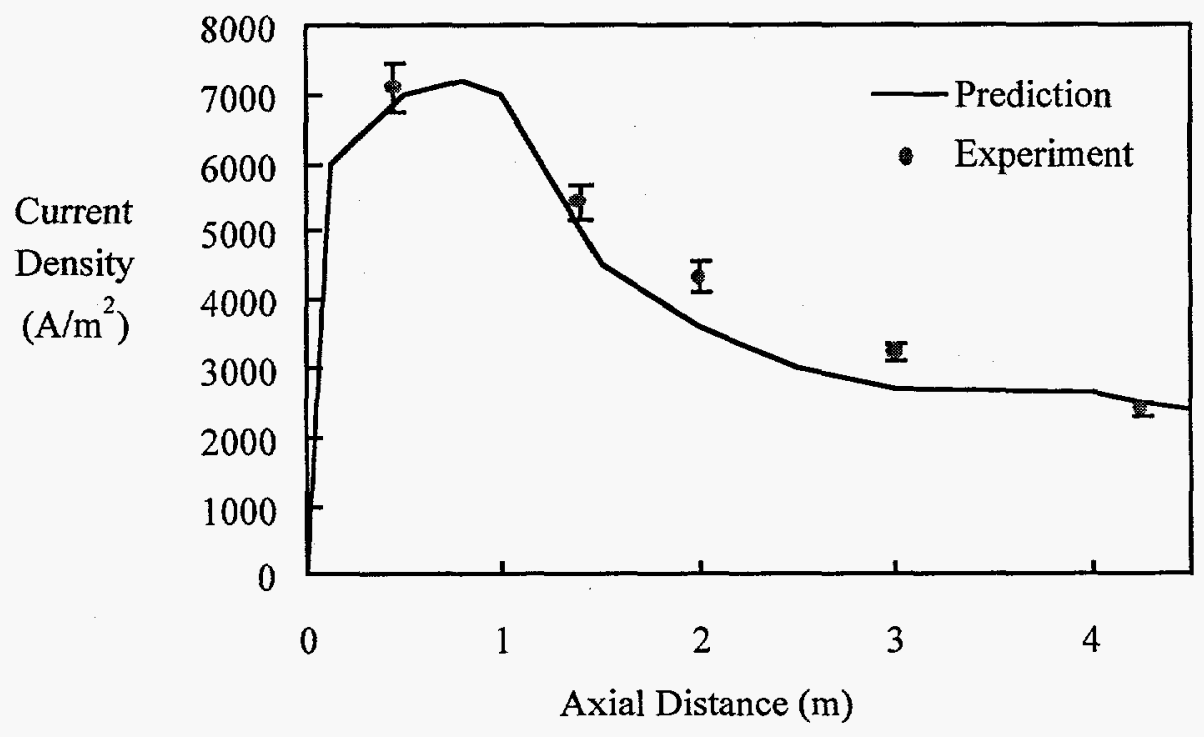

Figure 85 Computed and Measured Axial Distributions of Faraday Current Density

The slagging model described in the introduction of this section seems to capture the experimental characteristics of the channel. Its implementation in the MGMHD computer code was successful, with no numerical stability problems encountered. Results of these simulations indicated that the channel was likely not an appropriate design for the combustor because of the excessive cooling of the plasma and low channel performance. 


\section{SIMULATION OF THE INTEGRATED MHD SYSTEM}

The CDIF MHD power train system consists of four major components: a 50-MWt, twostage coal-fired combustor, a converging/diverging nozzle, a supersonic channel, and a diffuser. The combustor burns pulverized coal in two stages to achieve high temperature, while minimizing the pollutant $\mathrm{NO}_{\mathrm{x}}$ formation and removing most slag particles. By adding seed material to the high-temperature combustion flow, the fluid attains desired plasma conductivity for effective channel operations. After passing through the combustor, the plasma flow is accelerated in the nozzle to very high velocity (supersonic flow). The supersonic plasma flows into the MHD channel, in which electric power is extracted through electric-magnetic interactions. As the gas leaves the channel, the velocity is still high but pressure drops lower than atmospheric pressure. A diffuser is used to decelerate the flow and recover gas pressure from the kinetic energy. Overall performance of the power train system depends on the performance of each component; one needs to know the flow characteristics to evaluate the performance of each component. Computer simulation is an effective tool to learn the flow characteristics. It can enhance the understanding of the complex phenomena of flow hydrodynamics, combustion, droplet-gas interactions, and magnetic field interactions with the plasma flow. In addition, computational experiments can be conducted to evaluate the effects of design and operating parameters on the overall system performance and to explore potential improvements for continued development of the MHD system.

\subsection{Computer Codes for the System Simulation}

With extensive experience in CFD simulation, ANL used state-of-the-art computer codes for the flow simulation of the MHD power train system. Due to differences in the components of the power train system, different computer codes or different versions of a particular computer code are used to do the simulation of the combustor, the nozzle, the MHD channel, and the diffuser, respectively. The ICOMFLO code was used to predict flow patterns in the second-stage combustor. A two-phase compressible flow computer code derived from the ICOMFLO code was used for the converging nozzle. The MGMHD computer code was used to simulate the plasma flow in the MHD channel. A one-dimensional, compressible flow computer code was used for the diffuser flow. Table 18 summarizes major features of the four computer codes used for current MHD system simulation.

The computer codes for the combustor, the nozzle, and the channel were presented in previous chapters. The one-dimensional diffuser flow computer code is described briefly as follows. The diffuser consists of flat and diverging sections. The computer code solves the continuity, momentum, energy, and state equations for ideal reversible gas flows with heat loss using bridging relations to account for discontinuous flow changes across a normal shock wave. The solution algorithm of the diffuser computer code includes the following steps: (1) make an initial guess of the shock location, (2) compute the supersonic reversible flow properties before the shock location, the property change across the shock wave, the subsonic reversible flow properties after the shock location, (3) calculate heat losses through the diffuser walls, (4) check 
the computed exit pressure against the actual exit pressure, and (5) adjust shock location until the computed and the actual exit pressures match.

Table 18 Computer Codes for the MHD System Simulation

\begin{tabular}{|l|l|}
\hline \multicolumn{1}{|c|}{ MHD Component } & \multicolumn{1}{c|}{ Features of the Code } \\
\hline Second-Stage Combustor & Two-Dimensional \\
& Two-Phase \\
& Combustion \\
& Multi-Species \\
\hline Converging Nozzle & Two-Dimensional \\
& Two-Phase \\
& Combustion \\
& Compressible Flow \\
& Multi-Species \\
\hline Channel and Diverging Nozzle & Three-Dimensional \\
& Electric-Magnetic Interactions \\
& Current Leakage \\
\hline Diffuser & One-Dimensional \\
& Compressible Flow \\
& Shock Wave \\
\hline
\end{tabular}

\subsection{Flow Property Predictions of the Integrated MHD System}

The system simulation was used to predict the development of flow patterns in the MHD power train system at CDIF. The simulation integrated the calculations of the computer codes for the four MHD components. The computed flow properties at the combustor exit were used for the nozzle inlet; the computed flow properties at the nozzle exit were used for the channel inlet; and the computed flow properties at the channel exit were used for the diffuser inlet. Figure 86 shows the computed pressure, temperature, and gas velocity along the axial direction in the entire power train system. Note that axial distance $\mathrm{X}$ is measured from the entrance of the second-stage combustor. CDIF measured a heat loss rate of $1.4 \mathrm{MW}$ in the diffuser [41]. Assuming a diffuser exit pressure of $1 \mathrm{~atm}$, a normal shock is found occurring at $\mathrm{X}=7.6 \mathrm{~m}$, near the diffuser inlet. Gas pressure starts at $5.7 \mathrm{~atm}$ in the combustor, decreases to $3.5 \mathrm{~atm}$ in the nozzle, further decreases to $0.46 \mathrm{~atm}$ after the MHD channel, and finally recovers to $1 \mathrm{~atm}$ at the diffuser exit after the normal shock. Gas temperature increases from 1900 to $2800^{\circ} \mathrm{K}$ in the combustor, decreases to $2660^{\circ} \mathrm{K}$ at the nozzle throat, further decreases in the channel and the diffuser. Gas velocity increases from 50 to $180 \mathrm{~m} / \mathrm{s}$ due to the combustion in the combustor, accelerates to a sonic speed of about $900 \mathrm{~m} / \mathrm{s}$ at the nozzle throat, further accelerates to about $1500 \mathrm{~m} / \mathrm{s}$ in the divergent nozzle, remains above $1500 \mathrm{~m} / \mathrm{s}$ in the channel, and decelerates in the 
diffuser after the shock to about $200 \mathrm{~m} / \mathrm{s}$. At the diffuser inlet, the gas velocity is $1500 \mathrm{~m} / \mathrm{s}$, the pressure is $0.46 \mathrm{~atm}$, and the temperature is $2200^{\circ} \mathrm{K}$. The simulation predicted the channel performance, 1.2-MW power, 160-A load current, and $7700-\mathrm{V}$ load voltage. These predicted characteristics of the channel agree reasonably well with the nominal design characteristics $[40,41]$.

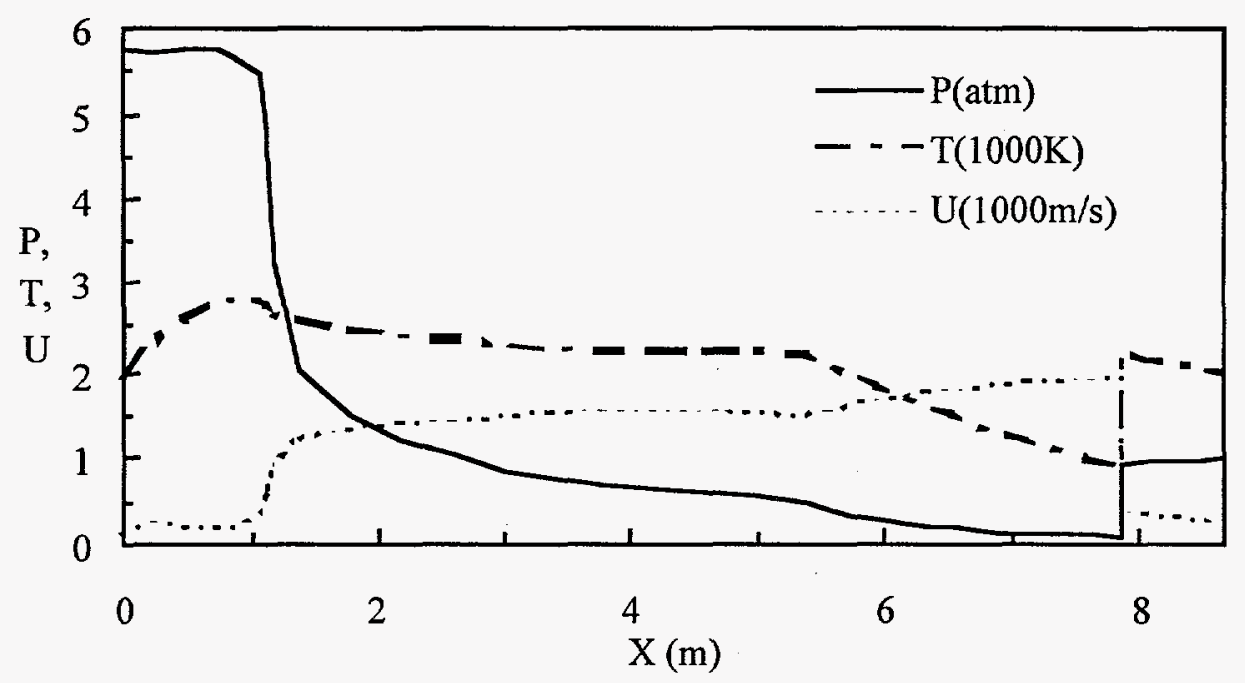

Figure 86 Axial Development of Flow Properties in the CDIF MHD Power Train 


\section{SUMMARY}

The adaptation and application of Argonne National Laboratory's (ANL's) computer codes to simulation and analysis of components of the MHD power train system at the Component Development and Integration Facility (CDIF) have been covered in this report, along with a presentation of significant findings in the form of data plots, comparison with experiment where comparison data were available, and considerable discussion of the simulation results as they relate to both individual component performance and overall MHD power train performance. The major components of the system included in the analysis were a 50-MWt, coal-fired twostage combustor and an MHD channel. The combustor, designed and built by TRW, includes a deswirl section between the first- and the second-stage combustors, and a converging nozzle following the second-stage combustor, which connects to the MHD channel. The first-stage swirl combustor was deemed a mature technology and, therefore, was not included in the computer simulation. Several versions of the ICOMFLO computer code, upgraded and adapted for specific component analysis, were used for the deswirl section, the second-stage combustor, and the converging nozzle leading to the MHD channel. The MGMHD code, upgraded with a slag current leakage submodel, was used for the MHD channel.

Although detailed boundary condition data, especially at interior component boundaries, can be very difficult to collect in high-temperature coal combustion environments (for example, quartz windows for optical measurement are rapidly covered with soot and slag), whatever experimental data were available were used judiciously during the course of the analysis project development in a variety of ways. Data from the test facilities were used to aid in calibrating parameters in the computer code, to validate the computer code, and to set base-case operating conditions for computations with the computer code. Extensive sensitivity and parametric studies were done on cold flow mixing in the second-stage combustor, reacting flow in the second-stage combustor and converging nozzle, and particle-laden flow in the deswirl zone of the first-stage combustor, the second-stage combustor, and the converging nozzle. These simulations with subsequent analysis were able to show clearly, in terms offlow patterns and various computable measures of performance, a number of sensitive and problematical areas in the design of the power train. The simulations of upstream components also provided inlet parameter profiles for simulation of the MHD power generating channel, the mean values of which were checked against published test data for reasonableness.

Among the more important results were the demonstration of the importance of oxidizer injection angle in the second-stage combustor. Injecting the oxidizer at an angle partially counter to the main flow direction produced the best mixing, the most efficient combustion, the most complete and early vaporization of potassium seed particles, and consequently, a plasma with the highest over conductivity and uniform distribution at the second-stage combustor exit. This counterflow injection angle was fortunately found to be in the same range for achieving all of the optimal conditions, between 120 and 140 degrees as measured from the downstream direction of the injector wall.

A need to optimize mean seed particle size was demonstrated. Seed particles that are too small clog injection nozzles. When seed particles are too large (about 100- $\mu \mathrm{m}$-diameter), 
vaporization does not reach completion during the residence time in the second-stage combustor, and the seed vapor does not have sufficient time to mix and form a uniform profile at the chamber exit.

A problem with seed- and slag-particle buildup in front of the oxidizer jets in the secondstage combustor was also identified. This problem was severe enough that the jets could become clogged in a matter of days or at most a couple of weeks, and CDIF confirmed that the problem was occurring at their test facility. No work was done on resolving this problem. The simulation in the deswirl zone with seed particle injection showed that particle trajectories, controlled primarily by momentum exchange with the gas flow via drag, are very sensitive to injection location, particle size, and injection velocity. All of these parameters must be optimized to minimize the rate at which seed particles collide with and build up on the deswirl section walls and to maximize the amount of seed that follows the main gas flow into the second-stage combustor.

Nozzle flow studies showed that much more uniform flow property development could be achieved in the nozzle area if the change rate with distance was adjusted to conform to the response rate of the flow at a particular position in the nozzle. A funnel-type, rapidly converging nozzle, originally used in the system design, produced a very nonuniform acceleration and pressure change rate, which was most rapid and primarily confined to the throat area. A problem of seed- and slag-particle collision with the nozzle walls that could result in rapid nozzle erosion was also uncovered. The y-component of gas velocity in the converging nozzle flow was simply not sufficient to change particle trajectories through drag to allow particles, which were not already nearly aligned with the nozzle throat, to follow the gas flow through the nozzle throat. Nearly the opposite problem was demonstrated with the cross-stream injection of iron oxide particles near the bottom of the side walls. These particles, when injected with the appropriate velocity, congregate in the nozzle center area and flow on into the channel. However, the primary process to spread them out again to cover the cathode wall is one of turbulent transport or diffusion, both relatively slow compared to the convective flow velocity, and therefore a uniform iron oxide coating of the cathode wall is difficult to achieve with this scheme.

In the MHD channel itself, simulation showed that continued supersonic expansion in the channel central region reduced temperature and consequently plasma conductivity to levels significantly lower than those necessary to achieve design goal performance.

In conclusion, the multicomponent computational fluid dynamic (CFD) studies of the MHD power train identified a number of optimal operating ranges for some parameters, such as oxidizer injection angle in the second-stage combustor, and also identified a number of areas for improvement in the current design in a way that clearly showed the physical mechanisms responsible for the difficulties. Future research could begin with this information as the basis for more detailed studies and investigation of alternatives that could greatly improve the performance of the overall MHD power-generating system. 


\section{REFERENCES}

1 "Open-Cycle Magnetohydrodynamic Electric Power Generation," M. Petrick and B. Ya. Shumyatsky, Eds., Joint Report of Argonne National Laboratory and U.S.S.R. Academy of Science (1978).

2 Chang, S.L., G.F. Berry, and N. Hu, "System Analysis of High Performance MHD Systems," Proc. of 23rd Intersociety Energy Conversion Engineering Conference, Denver, Colo., 4:455-459 (July 31-Aug. 5, 1988).

3 Grove, A., "Design Study of MHD Coal-Fired Combustor Second Stage," Proc. of 29th Symposium on Engineering Aspects of Magnetohydrodynamics, New Orleans, La., pp. III.3.1-III.3.9 (1991).

4 Braswell, R., T. Koyama, M. McAllister, S. Myrick, and B. Pote, "50 MWt Prototypical Combustor Performance," Proc. of 31 st Symposium of Engineering Aspects of Magnetohydrodynamics, Whitefish, Mont., pp. II.1.1-17 (1993).

5 Pian, C.P., and E.W. Schmitt, "Lessons Learned from the Design and Operation of the Integrated Topping Cycle MHD Generator," Proc. of 32nd Symposium on Engineering Aspects of Magnetohydrodynamics, Pittsburgh, Pa. (1994).

6 Albright, J., M. Bauer, R. Braswell, A. Grove, T. Koyama, S. Myrick, B. Pote, and H. Thom, "Demonstrated Performance of the TRW Pressurized Slagging Coal Combustor during the MHD POC Program," Proc. of 32nd Symposium of Engineering Aspects of Magnetohydrodynamics, Pittsburgh, Pa., (1994).

7 Hart, A.T., and D. Lofftus, "Coal-Fired MHD Test Progress at the Component Development and Integration Facility," Proc. of 32nd Symposium of Engineering Aspects of Magnetohydrodynamics, Pittsburgh, PA, Session 2.4 (1994).

8 Bouillard, J.X., J.L. Krazinski, S.P. Vanka, and G.F. Berry, User's Manual for MGMHD: $A$ Multigrid Three-Dimensional Computer Code for the Analysis of Magnetohydrodynamic Generators and Diffusors, Argonne National Laboratory Report ANL/MHD-89/1 (1989).

9 MSE, Inc., "Preliminary Test Report: 93-DVT-27," Butte, Mont., (1993).

10 R. Braswell, Private communication, TRW (June 1993).

11 Chang, S.L., S.A. Lottes, and M. Petrick, "Simulation of Three-Dimensional Multi-Phase Flow Characteristics in the Deswirl Section of the CDIF MHD Power Train," Proceedings of 32nd Symposium on Engineering Aspects of Magnetohydrodynamics, Pittsburgh, Pa., pp. 9.1-10 (1994).

12 Patankar, S.P., Numerical Heat Transfer and Fluid Flow, McGraw-Hill, (1980).

13 Launder, B.E., and D.B. Spalding, "The Numerical Computation of Turbulent Flows," Computer Methods in Applied Mechanics and Engineering, Vol. 3, pp. 269-289 (1974).

14 Chang, S.L., S.A. Lottes, and G.F. Berry, "Computation of Two-Dimensional NonReacting Jet/Main Flow Mixing in an MHD Second Stage Combustor," Numerical Heat Transfer, Part A, 20(2):223-236 (1991).

15 Wilson, W.W., D.V. Srikantaiah, and A. Grove, "Cold Flow Mixing Study of an MHD Combustor Model Using Laser Velocimeter and Concentration Measurements," Proc. of 28th Symposium on Engineering Aspects of Magnetohydrodynamics, pp. V.2.1-V.2.8, Chicago, Ill. (1990). 
16 Grove, A., "Cold Flow Mixing Study of 50 MWt MHD Coal-Fired Combustor," Proc. of 27th Symposium on Engineering Aspects of Magnetohydrodynamics, pp. 7.1.1-7.1.9, Reno, Nev. (1989).

17 Chang, S.L., S.A. Lottes, and G.F. Berry, "Two-Dimensional Non-Reacting Jet-Gas Mixing in an MHD Second Stage Combustor," Proc. of 28th Symposium on Engineering Aspects of Magnetohydrodynamics, pp. V.3.1-V.3.10, Chicago, Ill. (1990).

18 Lottes, S.A., and S.L. Chang, "Computer Simulation of Jet Penetration and Fluid Mixing in a Channel with Cross-Stream Jets," Proc. of the 7th International Conference on Advanced Science and Technology, pp. 188-198, Argonne, 111. (1991).

19 Lottes. S.A., and S.L. Chang, "Interactions of Turbulent Eddies and Combustion in an MHD Second Stage Combustor," Proc. of the 26th Intersociety Energy Conversion Engineering Conference, 3:69-74, Boston, Mass. (1991).

20 Berry, G.F., S.L. Chang, S.A. Lottes, and W.A. Rimkus, "Multi-Dimensional Computer Simulation of MHD Combustor Hydrodynamics," AIAA 22nd Fluid Dynamics, Plasma Dynamics \& Lasers Conference, Honolulu, Hawaii, AIAA 91-1511, (1991).

21 Lottes, S.A., and S.L. Chang, "Simulation of Combustion Processes in an MHD Second Stage Combustor," Proc. of 29th Symposium on Engineering Aspects of Magnetohydrodynamics, New Orleans, La., pp. III.4.1-11 (1991).

22 Chang, S.L., and S.A. Lottes, "Integral Combustion Simulation of a Turbulent Reacting Flow in a Channel with Cross-Stream Injection," Numerical Heat Transfer, Part A, 24(1):25-43 (1993).

23 Bittker, D.A., and V.J. Scullin, "General Chemical Kinetics Computer Program for Static and Flow Reactions, with Application to Combustion and Shock-tube Kinetics," NASA Report TN D-6586 (1972).

24 Pitz, W.J., and C.K. Westbrook, "Chemical Kinetics of the High Pressure Oxidation of nButane and Its Relation to Engine Knock," Combustion and Flame, Vol. 63, pp. 113-133, (1986).

25 Chang, S.L., S.A. Lottes, and J.X. Bouillard, "Dispersion of Seed Vapor and Gas Conductivity in an MHD Second Stage Combustor," Proc. of 30th Symposium on Engineering Aspects of Magnetohydrodynamics, Baltimore, MD, pp. VI.3.1-11, (1992).

26 Lottes, S.A., and S.L. Chang, "Particle-Jet Interactions in an MHD Second Stage Combustor," Proc. of 30th Symposium on Engineering Aspects of Magnetohydrodynamics, Baltimore, Md., pp. VI.4.1-12 (1992).

27 Bauman, L.E., and W. Wang, "Comparison of Faraday Rotation and Emission Absorption Measurements of Electron Density and Conductivity," Proc. of 29th Symposium of Engineering Aspects of Magnetohydrodynamics, New Orleans, La., pp. VI.4.1-13 (1991).

28 Benton, R.D., L.E. Bauman, R.A. Green, P.R. Jang, J.S. Lindner, D.V. Srikantaiah, W.W. Wilson, W.S. Shepard, and R.L. Cook, "Field Operations of the Diagnostic Instrumentation and Analysis Laboratory in Support of MHD Development," Proc. of 28th Symposium on Engineering Aspects of Magnetohydrodynamics, Chicago, Ill., pp. XII.1.1-11 (1990).

29 Frost, L.S., "Conductivity of Seeded Atmospheric Pressure Plasmas," J. Appl. Phys., 32:2029-2039 (1961). 
30 Chang, S.L., S.A. Lottes, J.X. Bouillard, and M. Petrick, "Study of Multi-Phase Flow Characteristics in an MHD Power Train," Proc. of 31st Symposium on Engineering Aspects of Magnetohydrodynamics, Whitefish, Mont., pp. Vb.2.1-12 (1993).

31 Bouillard, J.X., J.L. Krazinski, S.P. Vanka and G.F. Berry, User's Manual for the MGMHD: A Multigrid Three-Dimensional Computer Code for the Analysis of Magnetohydrodynamic Generators and Diffusers, Argonne National Laboratory Report ANL/MHD-89-1 (1989).

32 Rosa, R.J., "Magnetohydrodynamic Energy Conversion," McGraw-Hill, New York (1968),

33 Maxwell, C.D., D.M. Markham, S.T. Demetriades, and D.A. Oliver, Coupled Electrical and Fluid Calculations in the Cross Plane in Linear MHD Generators, Proc. of 16th Symposium on Engineering Aspects of Magnetohydrodynamics, Pittsburgh, Pa., pp. VII.3.13-VII.3.20 (May 1977).

34 Vanka, S.P., and R.K. Ahluwalia, "Three-Dimensional Flow and Thermal Development in Magnetohydrodynamic Channels," J. Energy, 6(3):218-221 (May-June 1982).

35 Vanka, S.P., R.K. Ahluwalia, and E.D. Doss, Three-Dimensional Analysis of MHD Generators and Diffusers, Argonne National Laboratory Report ANL/MHD-82-4 (March 1982).

36 Rosa, R., "Leakage Resistance and Current Inferred from CDIF Operating Data," personal communication (undated).

37 Rosa, R., "Leakage Currents and Electrode Wear Processing," 29th Symp. on Engineering Aspects of Magnetohydrodynamics, pp. VIII:1-14, New Orleans, La. (1991).

38 Rosa, R., and J. Pollina, Corrosion and Arc Erosion in MHD Channels, Final Report DEFG2288PC.88928 (1992).

39 Pollina, R., et al., "Topical Report on the Electrical Behavior of Rosebud Coal Slag under MHD Conditions," Proc. of 17th Symposium on Engineering Aspects of Magnetohydrodynamics" (1978).

40 Lineberry, J.T., Y.C.L. Wu, and G.L. Nelson, "Analysis of Coal-Fired Generator Experiments at the CDIF," Proc. of 28th Symposium on Engineering Aspects of Magnetohydrodynamics, Chicago, ILL., pp. IV.1-IV.6-16 (1990).

41 Petty, S.W., A. Dunton, J. Reich, and W. Simpson, "1 $\mathrm{A}_{4}$ Channel Design and Fabrication," Proc. of 29th Symposium on Engineering Aspects of Magnetohydrodynamics, New Orleans, La., pp. II.1.1-II 1.10 (1991).

42 MSE, Inc., "CDIF Test Report Data," Butte, Mont., (1993). 